\title{
CHARACTERIZING PSYCHOBEHAVIORAL RISKS IN SURVIVORS OF MULTIPLE PRIMARY CANCERS
}

\author{
by \\ Sarah M. Belcher \\ Bachelor of Science in Nursing, The Ohio State University, 2002
}

Submitted to the Graduate Faculty of the

School of Nursing in partial fulfillment

of the requirements for the degree of

Doctor of Philosophy

University of Pittsburgh

2018 


\title{
UNIVERSITY OF PITTSBURGH \\ SCHOOL OF NURSING
}

This dissertation was presented

by

\author{
Sarah M. Belcher
}

It was defended on

June 25, 2018

and approved by

Susan M. Sereika, Professor, School of Nursing, Department of Health \& Community Systems Paula S. Sherwood, Professor, School of Nursing, Department of Acute and Tertiary Care Dana H. Bovbjerg, Professor, School of Medicine, Department of Psychiatry Grace B. Campbell, Assistant Professor, School of Nursing, Department of Acute \& Tertiary Care

Dissertation Advisor: Heidi S. Donovan, Professor, School of Nursing, Department of Health \& Community Systems 
Copyright $(\subset$ by Sarah M. Belcher

2018 


\section{CHARACTERIZING PSYCHOBEHAVIORAL RISKS IN SURVIVORS OF MULTIPLE PRIMARY CANCERS \\ Sarah M. Belcher, PhD, RN, OCN ${ }^{\circledR}$ \\ University of Pittsburgh, 2018}

Background: As the population ages and cancer survival improves, the incidence of multiple

primary cancers (MPC) is increasing. Several studies have documented poorer health outcomes among adults with MPC compared to single-cancer survivors. However, there is a paucity of research focused on understanding factors linking MPC to poor health outcomes and identifying individual (e.g., personality, sociodemographic, clinical) factors that increase risk.

Purpose: The purpose of this study was to test a psychobehavioral stress-response model to identify factors associated with MPC health outcomes. We aimed to: 1) test the hypothesized model, examining linear relations among six latent variables: perceived stress, psychological response, behavioral response, financial toxicity, social health, and physical health; 2) explore associations between individual characteristics and upstream latent variables; and 3) describe self-management behaviors of MPC survivors.

Methods: This cross-sectional study included MPC survivors recruited through a regional tumor registry whose first cancers (stages I-III) were diagnosed within 1-10 years. Data were collected via 1) a battery of validated questionnaires to measure latent variables and covariates; 2) tumor registry records; and 3) medical records. Structural equation modeling was performed to fit and modify the measurement model, specify the full SEM, and identify significant covariates. Descriptive statistics were conducted to characterize self-management. 
Results: 211 participants completed questionnaires. Data fit a four-factor modified measurement model linking self-management, distress, financial toxicity, and functional health. Overweight BMI, graduate education, less neuroticism, and increased social support predicted better self-management. Poorer self-management, greater neuroticism, and lower social support predicted increased distress. Greater distress predicted financial toxicity. Greater distress and financial toxicity predicted poorer functional health. Scores for positive selfmanagement were generally high; obesity rates were above published norms.

Conclusions: MPC survivors with higher risk BMI, less education, greater neuroticism, and lower social support should be considered at risk for poorer self-management and negative health outcomes. Self-management behaviors and distress are potentially modifiable intervention targets to reduce financial toxicity and improve functional health. Future research should evaluate the model with a focus on developing the science of MPC self-management and financial toxicity and include longitudinal assessments to identify critical times of increased vulnerability during MPC survivorship. 


\section{TABLE OF CONTENTS}

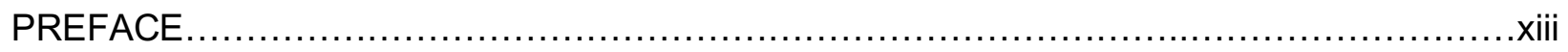

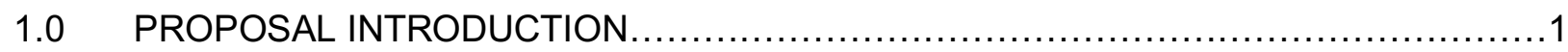

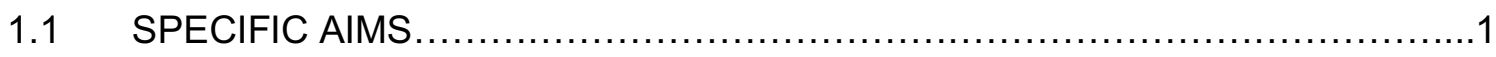

1.2 BACKGROUND, SIGNIFICANCE, and INNOVATION ...........................

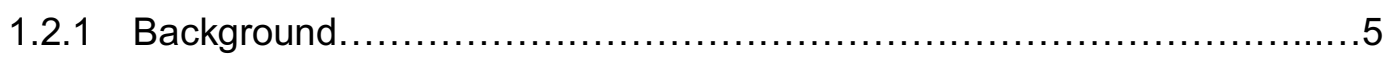

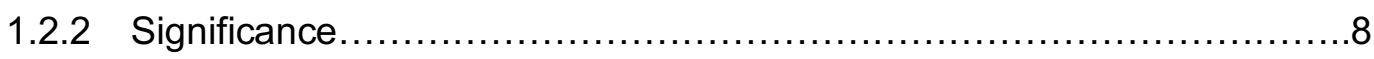

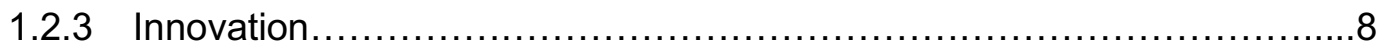

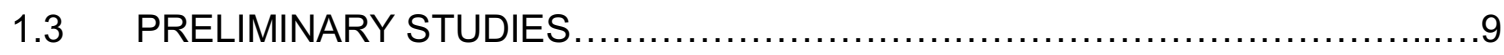

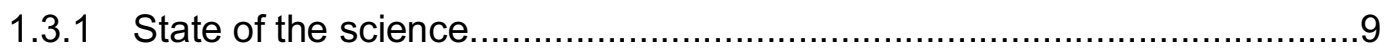

1.3.2 Expansion of conceptual model......................................................10

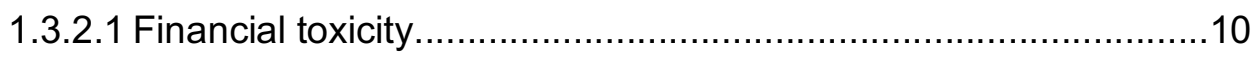

1.3.3 Conceptualization of key model concepts..........................................11

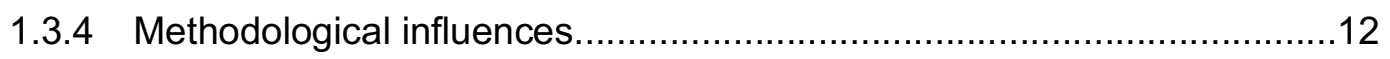

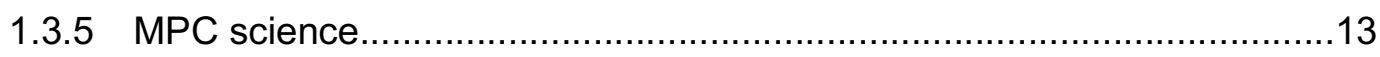

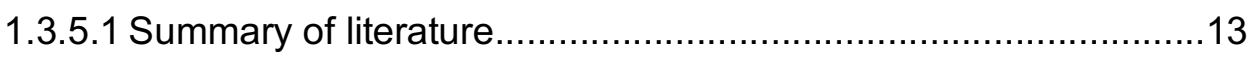

1.3.5.2 Gaps in the science and future directions.................................13

1.4 RESEARCH DESIGN AND METHODS ....................................... 14

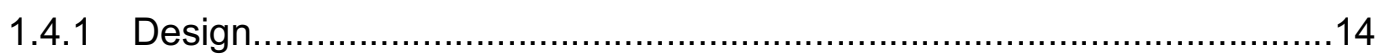

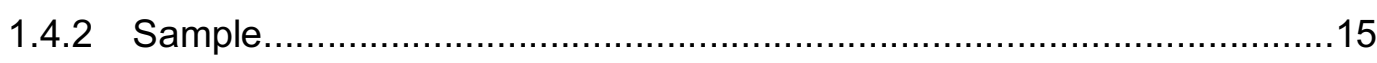

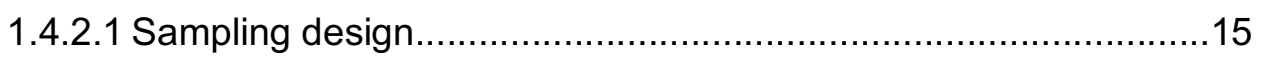

1.4.2.2 Sampling frame and elements................................................. 15

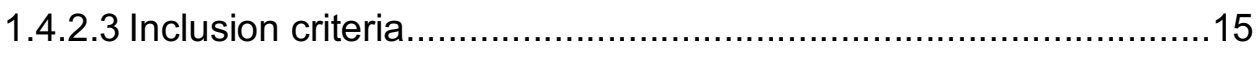

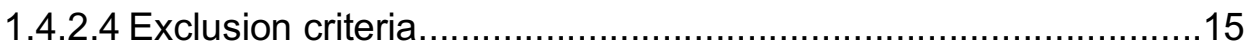

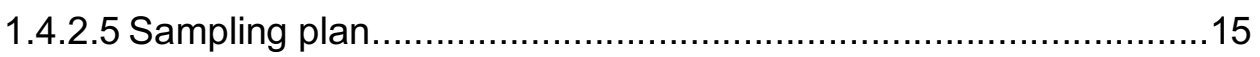


1.4.3 Recruitment and data collection procedures..................................16

1.4.3.1 Dillman's Tailored Design Method....................................17

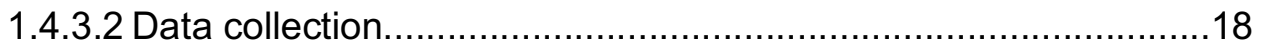

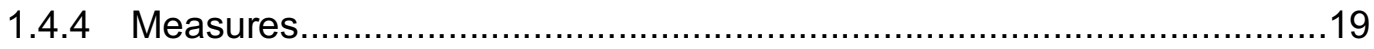

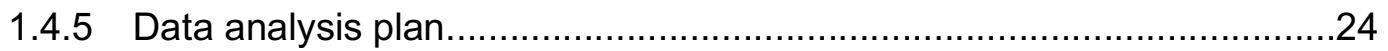

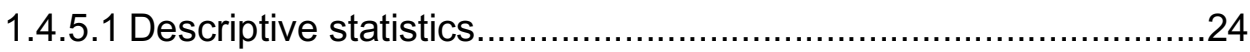

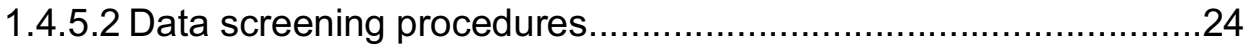

1.4.5.3 Data analysis procedures............................................25

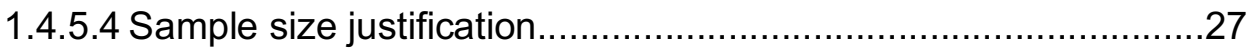

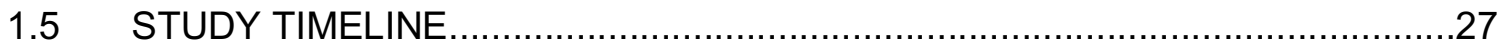

1.6 POTENTIAL LIMITATIONS AND ALTERNATIVE APPROACHES ...................29

1.7 PUBLICATIONS RELEVANT TO THE PROPOSED RESEARCH..................29

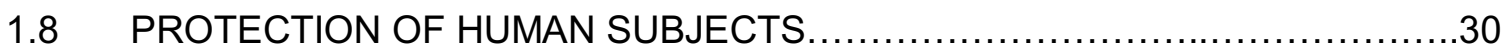

1.8.1 Human subjects involvement and characteristics..........................30

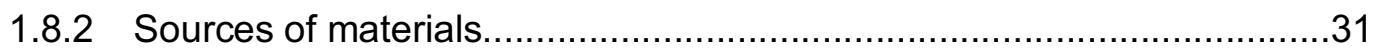

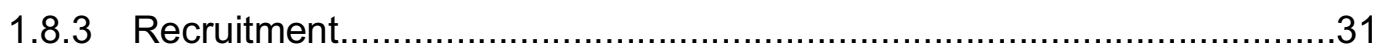

1.8.4 Potential risks and adequacy of protection against risks................33

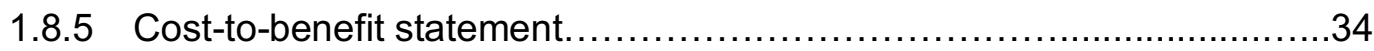

1.8.5.1 Importance of knowledge to be gained...............................34

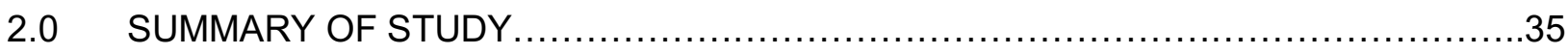

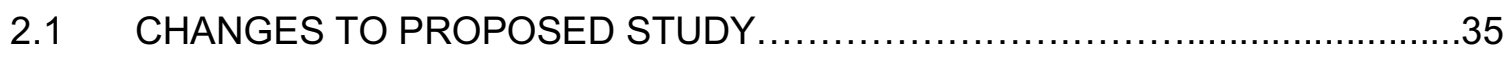

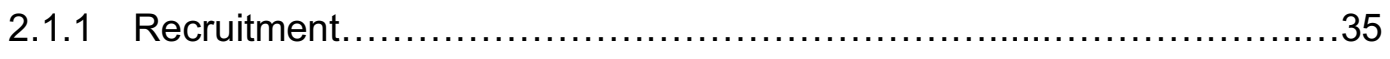

2.1.1.1 Additional participation criteria.........................................35

2.1.1.2 Dillman's Tailored Design Method........................................36

2.1.1.3 Nonresponse and return to sender.....................................3

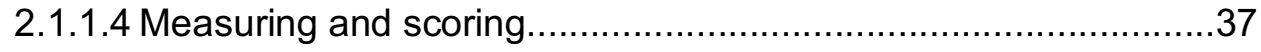


2.1.1.4.1 Psychological responses....................................37

2.1.1.4.2 Physical health outcomes....................................37

2.1.1.4.3 Personality........................................................

2.1.1.4.4 Exploratory measures.............................................

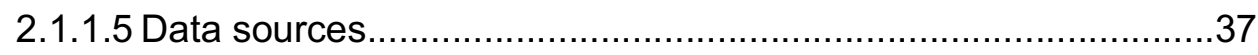

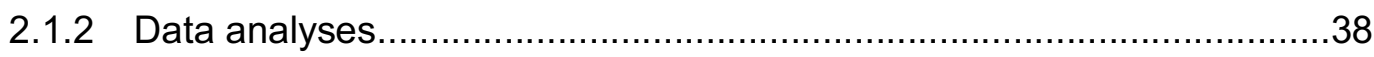

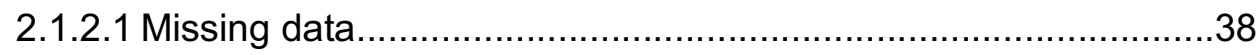

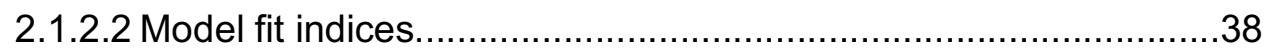

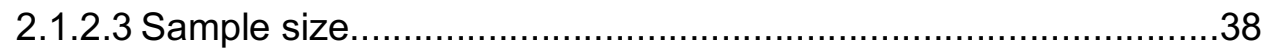

2.1.2.4 Analytic variables for multivariate analyses................................38

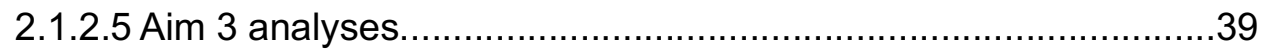

2.2 CONCLUSIONS, IMPLICATIONS FOR NURSING, AND FUTURE STUDIES...39

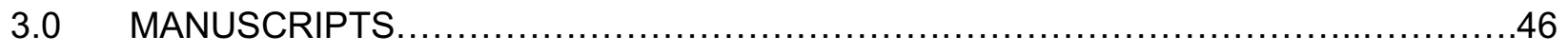

3.1 DISSERTATION MANUSCRIPT 1: REVIEW OF LITERATURE ...................47

3.2 DISSERTATION MANUSCRIPT 2: PILOT TESTING ASSOCIATIONS AMONG

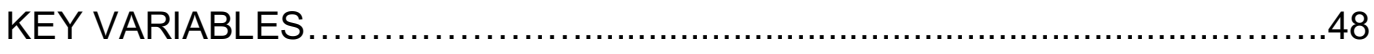

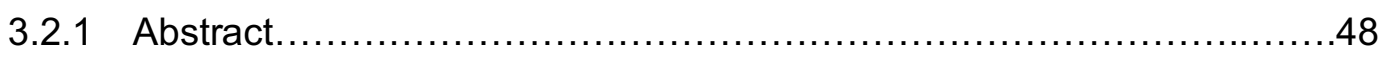

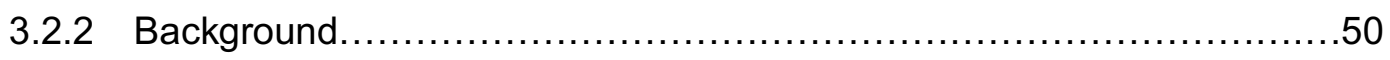

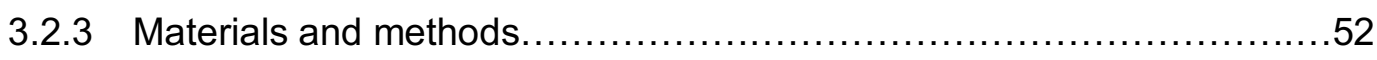

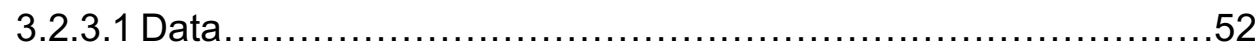

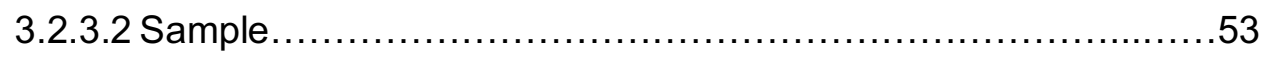

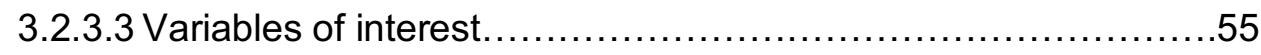

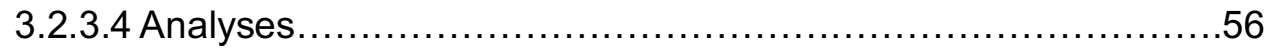

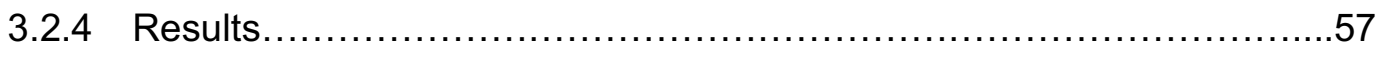

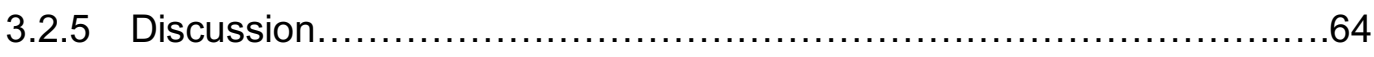

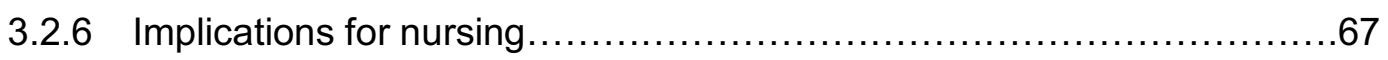




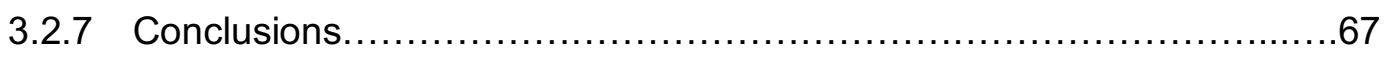

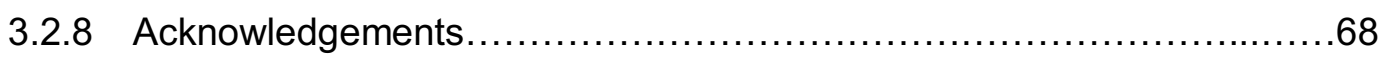

3.3 DISSERTATION MANUSCRIPT 3: MODEL TESTING TO CHARACTERIZE

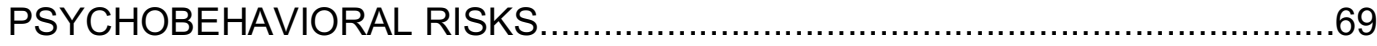

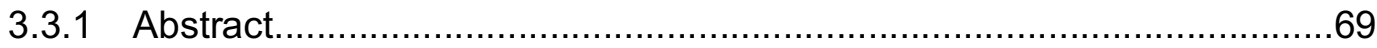

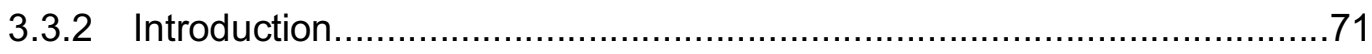

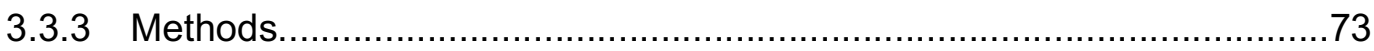

3.3.3.1 Sample and setting ...................................................................

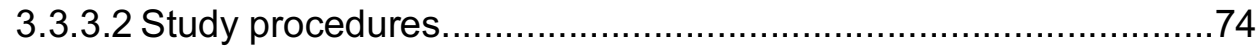

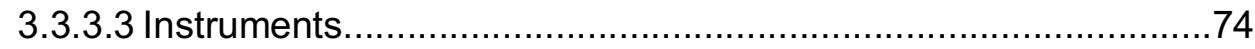

3.3.3.3.1 Sociodemographic, personal, and clinical predictors...75

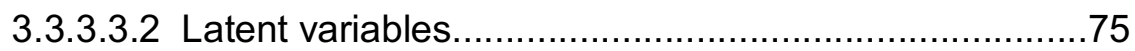

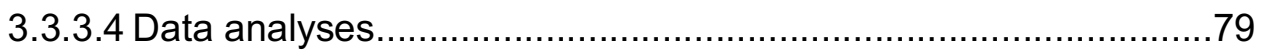

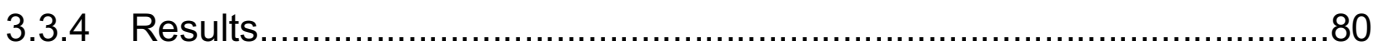

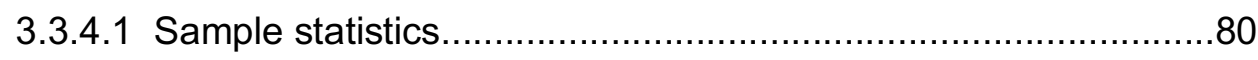

3.3.4.2 Description of key variables in the measurement model..............85

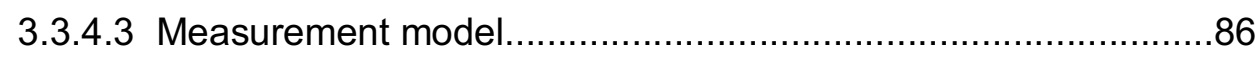

3.3.4.3.1 Model fit and parameter estimation..............................90

3.3.4.4 Full structural equation model..............................................92

3.3.4.4.1 The hypothesized model............................................92

3.3.4.4.2 Model fit and parameter estimation..............................93

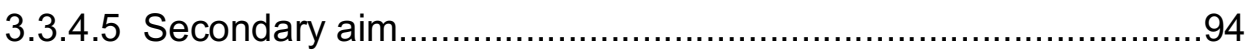

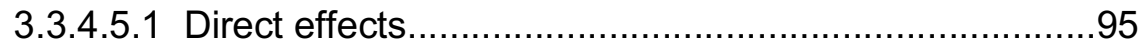

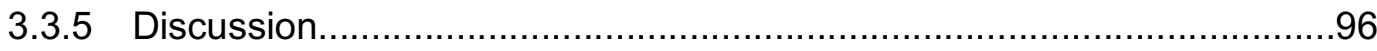

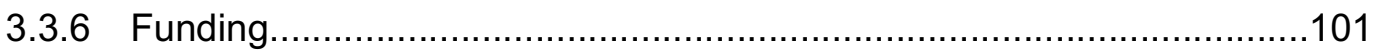

3.3.7 Conflict of interest disclosures........................................................101 


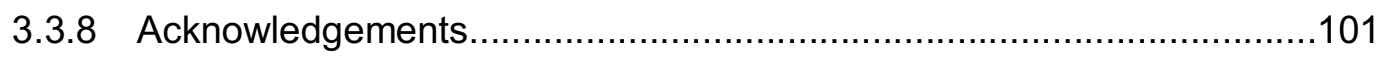

3.3.9 Supplementary online material....................................................... 102

4.0 DESCRIPTION OF SELF-MANAGEMENT BEHAVIORS.....................................108

4.1 THE IMPORTANCE OF SELF-MANAGEMENT BEHAVIORS ........................108

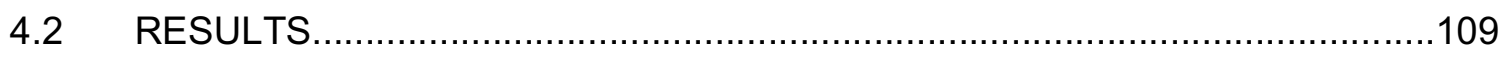

4.2.1 Self-management domain scores...................................................109

4.2.2 Self-management behavior item scores.............................................110

4.2.3 Indicators of negative health behaviors.............................................111

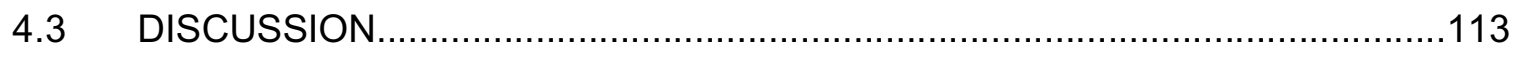

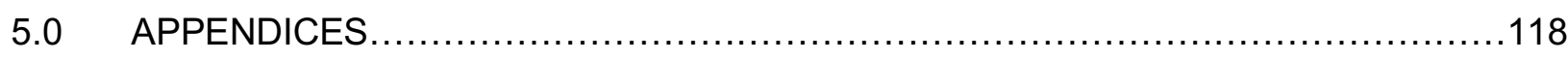

APPENDIX A: STUDY RECRUITMENT MATERIALS AND REFUSAL FORM .....................119

APPENDIX B: STUDY COVER LETTER AND INSTRUMENTS ...............................120

APPENDIX C: HUMAN SUBJECTS APPROVALS AND CONSENTS ............................121

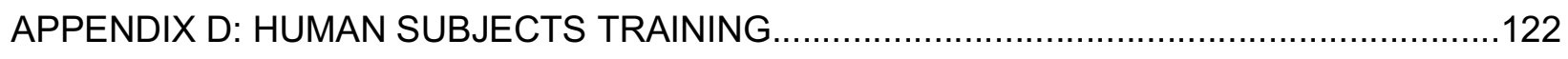

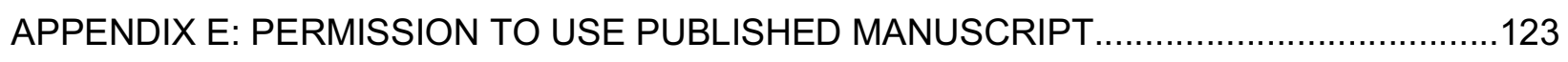

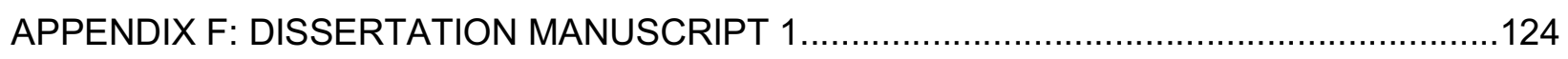

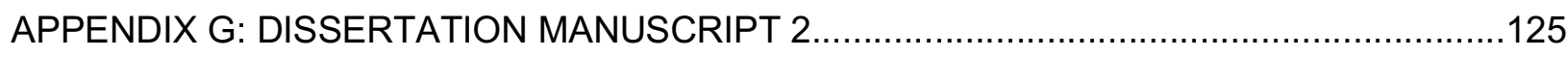

APPENDIX H: COVER LETTER FOR UNPUBLISHED MANUSCRIPT 3 ...........................127

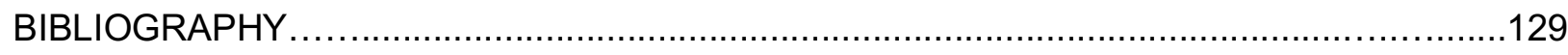




\section{LIST OF TABLES}

Table 1: Dissertation Study Concepts, Measures, Levels of Measurement, and Psychometric Properties.

Table 2: $\quad$ Study Timeline.

Table 3: $\quad$ Sample Sociodemographic and Clinical Characteristics by Single Cancer and Multiple Primary Cancer Groups.....

Table 4: $\quad$ Mean Sum Scores on Outcome Category Scales by Single and Multiple Primary

Cancer Groups

Table 5: Multivariate Linear Regression Models of Psychological Distress, Healthy Lifestyle, Positive Healthcare Utilization and Benefit Finding "As a Result of Having Cancer"

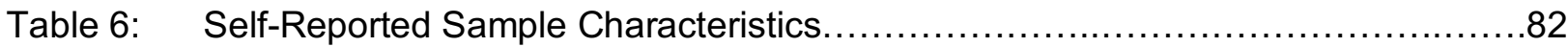

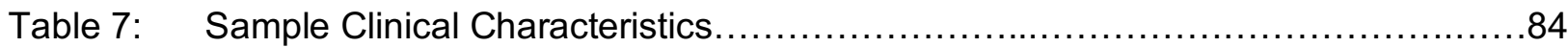

Table 8: $\quad$ Scores for Variables in the Originally Hypothesized Measurement Model...........85

Table 9: $\quad$ Frequencies of Second Cancer Diagnosis Sites..........................................102

Table 10: Frequencies of Sample First or Second Cancer Diagnoses Sites................103

Table 11: Sample Patterns of First and Second Cancer Diagnoses Sites..................104

Table 12: Most Common Second Cancer Sites within Each First Primary Cancer Site Group

Table 13: Sociodemographic and Clinical Comparisons of Cancer Registry Sociodemographic and Clinical Data: Participants versus Nonparticipants.

Table 14: Raw Scores for Self-Management Domains, as Measured by Health Education Impact Questionnaire Measure Subscales.

Table 15: Frequencies of Participants who Agree with Performing Self-Management Behaviors, by Domain and Item.

Table 16: Sample Characteristics of Modifiable Health Behaviors 


\section{LIST OF FIGURES}

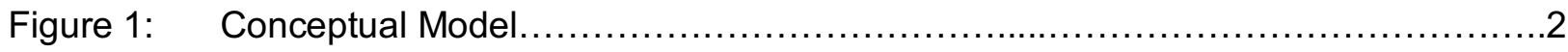

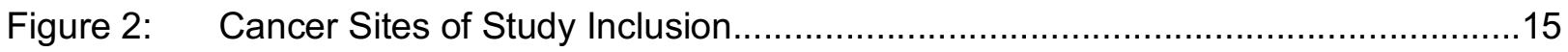

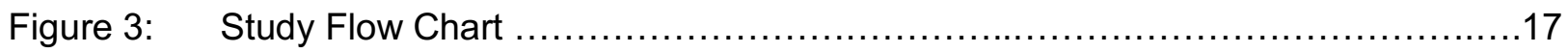

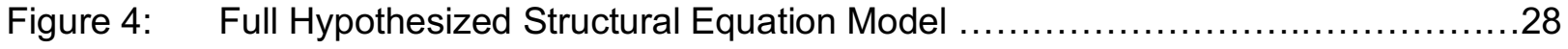

Figure 5: $\quad$ Flow Chart of Process Resulting in Post-Treatment Adult Cancer Survivor Sample

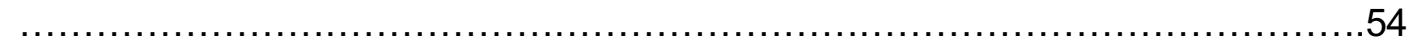

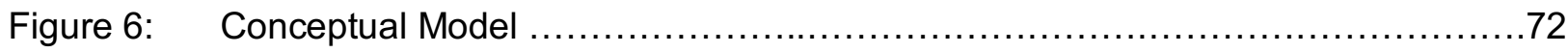

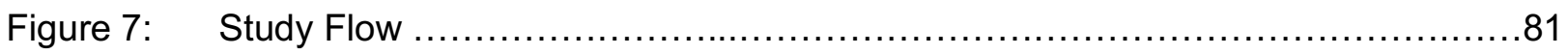

Figure 8: $\quad$ Originally Hypothesized Six-Factor Measurement Model ......................88

Figure 9: $\quad$ Modified Four-Factor Measurement Model................................. 89

Figure 10: $\quad$ Modified Four-Factor Measurement Model ................................91

Figure 11: Hypothesized Full Structural Equation Model Based on Modified Measurement

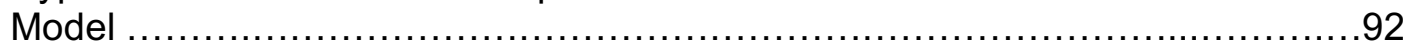

Figure 12: Final Modified Structural Equation Model Based on Modified Measurement Model

Figure 13: Final Parsimonious Structural Equation Model Based on Modified Measurement Model. 


\section{PREFACE}

Thank you to the LAMP Study participants who gave generously of your time to make this dissertation study possible. Your experiences make you shared experts in this research.

I am also endlessly thankful for my esteemed committee members for sharing your expertise and supporting this work. Thank you to: Dr. Heidi Donovan, my committee chair, for identifying the researcher in me and for your fearless mentoring in both science and humanity;

Dr. Susan Sereika for your individualized approach to my training and research and for your ongoing encouragement and tireless support when I was in the weeds; Dr. Paula Sherwood for your shared excitement about my work and for being the voice in my head challenging me to conduct research that matters ("so what?"); Dr. Dana Bovbjerg for your cross-disciplinary mentorship and support of nursing science; and Dr. Grace Campbell for your example of science that is guided by the participants you seek to serve. I also recognize the late Dr. Susan Cohen and Dr. Catherine Bender for your expert instruction, mentorship, and support of my training and research. Also priceless have been the peer-mentorship, support, and friendships gained from past and current members of Dr. Donovan's Women's Cancer Research Group and my classmates. Thank you, too, to the UPMC Cancer Network Registry, physician champions, and undergraduate students (Emilie Hausmann, Raegan Kramer, Olivia D’Antonio, and Katie Corey) who supported this research.

Thank you to my family and friends for your unending support, recognition of the effort and drive required to reach this point, and for your encouragement along the way. Thank you specifically to my family for making sacrifices to make this possible and to my children, Ella Katelyn and Aiden Titus, for providing me with inspiration each step of the way.

I have been so fortunate throughout my career to have had instructors, mentors, peers, interdisciplinary colleagues, preceptees, students, and patients who have been foundational in challenging me to strive towards excellence and develop as a leader. They have impressed upon me the great privilege it is to touch peoples' lives as a nurse. I would be remiss to not 
specifically name nursing mentors who have so gracefully left their mark on my career, including Danette Birkhimer, Sandy Scheiner, the James nurses (too many to list), Kelly TomlinsonPinkham, and Wendy Miano.

I acknowledge gratefully the following support that made this dissertation study possible:

Robert Wood Johnson Foundation Future of Nursing Scholars; American Cancer Society Doctoral Degree Scholarship in Cancer Nursing (DSCNR-17-077); Nightingale Awards of Pennsylvania PhD Scholarship; and funding through the University of Pittsburgh School of Nursing, including the Margaret E. Wilkes Scholarship Fund Award, the Newmeyer-Thompson Doctoral Student Award, and the Bessie Li Sze Scholarship Fund Award. 


\subsection{PROPOSAL INTRODUCTION}

\subsection{SPECIFIC AIMS}

As the cancer survivor population ages and cancer survival improves (American Cancer Society, 2017; Bluethmann, Mariotto, \& Rowland, 2016; Jemal et al., 2017), the incidence of subsequent primary cancer diagnoses in cancer survivors is increasing. Affecting approximately 3 million Americans, nearly one in five cancers diagnosed in the United States occurs in an individual with a previous cancer diagnosis (Morton, Onel, Curtis, Hungate, \& Armstrong, 2014). These multiple primary cancers (MPC), $\geq 2$ histologically distinct primary cancer diagnoses that have been ruled out as being metastatic disease (Begg, 1999), are a leading cause of morbidity and mortality among cancer survivors. Previous large sample studies have established that MPC survivors, compared to single cancer survivors and healthy (no cancer) controls, have increased risk for poor health outcomes including psychological distress, risky health behaviors, and negative physical health outcomes (Andrykowski, 2012; Burris \& Andrykowski, 2011; Dowling et al., 2013; Gotay, Ransom, \& Pagano, 2007; Thong et al., 2013).

Lazarus and Folkman classically defined perceived stress as the perception that one's demands exceed his or her coping resources (Lazarus \& Folkman, 1984). Cancer patients face demands throughout the cancer continuum that may be perceived as exceeding their coping resources (e.g., cancer diagnosis, treatment, and/or persistent symptoms). Resulting perceived stress can produce a cascade of psychological, behavioral, and biologic responses that negatively impact health outcomes (Bode, Hahn, Devellis, \& Cella, 2010). The proposed conceptual model for this study (see Figure 1) is informed by biobehavioral frameworks, which have contributed to research identifying pathways linking stress to poor outcomes (Andersen, Kiecolt-Glaser, \& Glaser, 1994; Andersen et al., 2008; Bower \& Lamkin, 2013; Cohen, Kessler, \& Underwood Gordon, 1995; Sherwood et al., 2008). This study will be the first to apply this type of framework to MPC research, providing opportunities to 1) guide early identification of 
MPC survivors most vulnerable to persistent stress and 2) identify important pathways (i.e., possible intervention points) that contribute to the cascade of negative health outcomes observed in this patient population (Grady \& Gough, 2014; Grey, Knafl, \& McCorkle, 2006; Grey, Schulman-Green, Knafl, \& Reynolds, 2015).

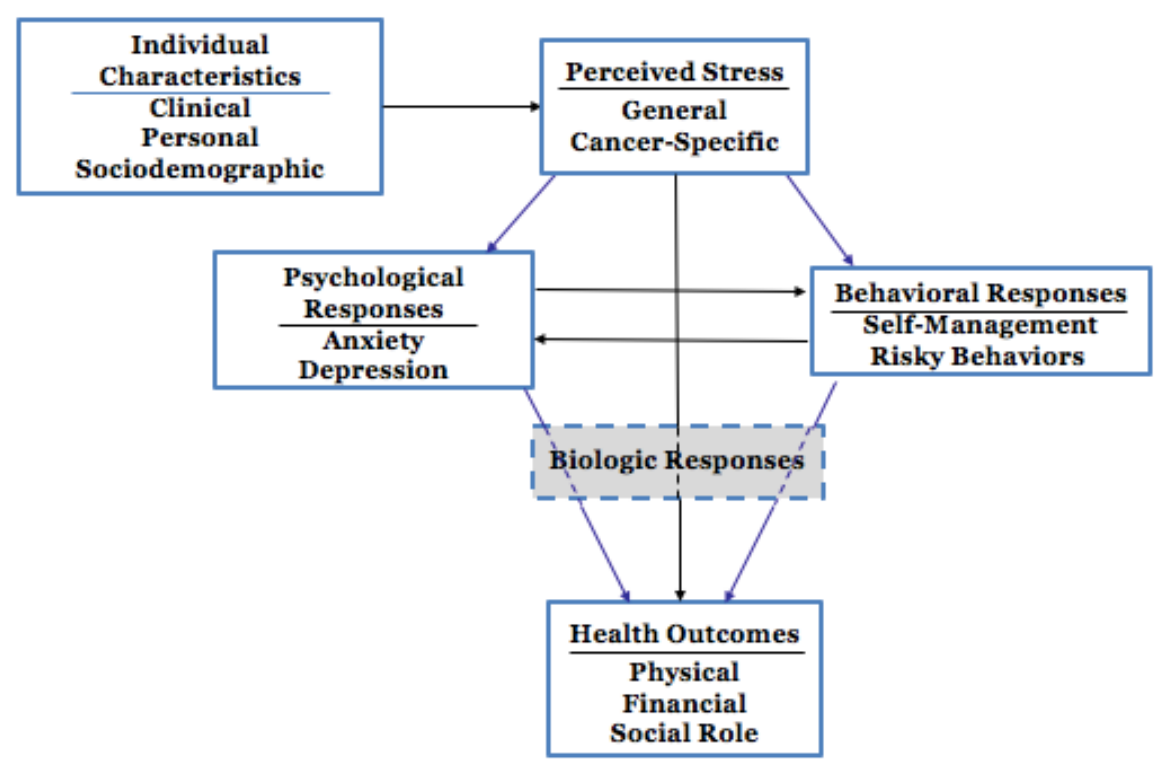

Figure 1. Conceptual model. This figure depicts the cascade of psychological, behavioral, and biologic responses to perceived stress that can negatively impact health outcomes in adults with multiple primary cancers. Biologic responses and pathways will be investigated in future study.

Our preliminary work, building on early MPC literature, has demonstrated that, compared to single cancer survivors, adults with MPC generally experience higher levels of stress (Gotay et al., 2007), report poorer psychological well-being (Andrykowski, 2012; Belcher et al., 2017; Belcher, Hausmann, Cohen, Donovan, \& Schlenk, 2016; Burris \& Andrykowski, 2011; Gotay et al., 2007; Thong et al., 2013), engage in more negative health behaviors (Burris \& Andrykowski, 2011), and are at increased risk for poor health outcomes (Andrykowski, 2012; Belcher et al., 2015; Dowling et al., 2013; Gotay et al., 2007; Thong et al., 2013). Our work also complements previous findings that MPC survivors live with cancer as a chronic illness (Belcher et al., 2017; Gotay et al., 2007; Thong et al., 2013). Importantly, prolonged perceived stress has been shown 
to increase susceptibility to negative physical health outcomes (Cohen et al., 1995; Cohen, n.d.; G. E. Miller, Cohen, \& Ritchey, 2002; O'Connor et al., 2009). However, little is known about the characteristics that put individual MPC survivors at risk for prolonged perceived stress or the psychological and behavioral pathways that could serve as targets for self-management interventions to improve health outcomes in MPC survivors.

This proposed conceptual model is informed by several biobehavioral frameworks, which have contributed to research identifying pathways linking stress to poor health outcomes (Andersen et al., 1994; Andersen et al., 2008; Bower \& Lamkin, 2013; Cohen et al., 1995; Sherwood et al., 2008). However, these models have not yet been applied to MPC research. Important weaknesses in MPC survivorship research to date include: 1) a lack of attention to who among the many survivors of MPC are at increased risk for poor health outcomes; 2) inattention to consistent, valid perceived stress measurement; and 3) an almost exclusive focus on negative behavioral responses without attention to positive behavioral responses (i.e., selfmanagement). Robust self-management literature in chronic illness (Grady \& Gough, 2014; Grey et al., 2015; Lorig \& Holman, 2003) and general cancer (Chen et al., 2015; Hammer et al., 2015; McCorkle et al., 2011; Miller, Bowen, Croyle, \& Rowland, 2009; Risendal et al., 2015) supports the premise that identification of characteristics of MPC survivors most at risk of poor health outcomes and factors that promote positive self-management behavioral responses will advance MPC science toward early risk identification and interruption of negative stress response pathways. It has been demonstrated in the literature that MPC survivors are at increased risk for poor health outcomes compared to their single cancer counterparts. This study will advance the science to begin to elucidate who among MPC survivors is at greatest risk and why.

My long-term goal is to develop a program of cancer survivorship research focused on the development of strategic self-management interventions to interrupt biobehavioral stress response pathways in at-risk survivors of MPC. The overarching purpose of this dissertation 
study, and the first step in my program of research, is to test the proposed conceptual model to better understand the components that impact health outcomes in persons with a history of MPC diagnoses. This study aims to answer the following research question: Does the proposed conceptual model explain poor health outcomes in persons with MPC? Our central hypothesis is that survivors of MPC are exposed to a wide range of cancer- and treatmentrelated stressors that are associated with persistent perceived stress and result in a cascade of psychological (e.g., depression, anxiety), behavioral (i.e., lack of positive self-management and risky behaviors), and biologic responses that are associated with poor health outcomes (i.e., physical, financial, and social). The rationale underlying the proposed research is that positive self-management behaviors have the potential to interrupt these negative pathways and to reduce negative impact after an MPC diagnosis. By elucidating mechanisms and identifying characteristics of MPC survivors with high risk for poor health outcomes, findings from this study will provide the basis for future studies that will include development of targeted selfmanagement interventions, interventions to trigger appropriate screening in high risk MPC patients, and incorporate important biological pathways known to impact health outcomes.

Specifically, in a cross-sectional sample of $n=440$ adults with a history of multiple (i.e., two or more) primary cancers, we will pursue the following specific aims:

Aim 1: Determine whether the proposed conceptual model fits data collected from MPC survivors.

Aim 1a: Evaluate whether the proposed overall model adequately explains variation in health outcomes among adults with MPC.

Aim 1b: Evaluate whether post-hoc model modifications based on previous cancer literature and sample data improve model fit and parsimony.

Exploratory Aim 2: Identify additional individual characteristics (i.e., clinical, personal, and sociodemographic) that contribute to improved model fit.

Exploratory Aim 3: Describe the self-management behaviors used by MPC survivors. 


\subsection{BACKGROUND, SIGNIFICANCE, AND INNOVATION}

\subsubsection{Background}

The National Academy of Medicine, the American Cancer Society, cancer survivorship advocates, clinicians, and scientists have called for an increased focus on addressing the health and psychosocial needs of cancer survivors (American Cancer Society, 2016; Klein et al., 2014; Miller et al., 2016; Mullan, 1985, 2016; National Academy of Sciences, 2006), and survivors of MPC represent a growing, understudied, and at-risk group in critical need of additional research.

The current number of cancer survivors living in the United States, 15.5 million, is projected to increase to 26.1 million by 2040 , and the cancer survivor population is aging (American Cancer Society, 2017; Bluethmann et al., 2016; Jemal et al., 2017). With improved survival and increased age, however, comes the risk of developing a wide range of late effects of cancer and cancer treatment including the development of second cancers. As compared to those in the general population without a cancer diagnosis, cancer survivors face a $14 \%$ higher risk of developing a new primary cancer (Fraumeni, Curtis, Edwards, \& Tucker, 2006). The observed increased risk is likely attributable to complex interactions among risk factors, including factors ranging from age at, and site of, first diagnosis, carcinogenic effects of cancer treatment, and genetic susceptibility (most pronounced in childhood survivors) to cumulative environmental exposures and lifestyle factors (e.g., smoking, alcohol use) in adult survivors (American Cancer Society, 2016; Fraumeni et al., 2006; Morton et al., 2014). Cancers specifically linked to the risk of subsequent cancer diagnoses include Hodgkin's lymphoma, non-Hodgkin's lymphoma, select solid tumors (i.e., prostate, testicular, ovarian, breast, and cervical), and childhood cancers (American Cancer Society, 2012; Meadows et al, 2009). The National Cancer Institute reports that one in five cancers diagnosed in the United States will occur in someone who has a previous cancer diagnosis, and MPCs are a major cause of morbidity and mortality in cancer survivors (De Gonzalez et al., 2011; Morton et al., 2014; National Cancer Institute, n.d.-a). 
Diagnoses, disease- and treatment-related effects, and care transition points (e.g., transition to survivorship, recurrence, and end of life) commonly result in varying levels of distress for cancer patients (National Comprehensive Cancer Network, 2015); 20-47\% of cancer patients with new and recurrent cancer diagnoses experience significant distress (Holland et al, 2013). Literature from various countries estimates mood disorders are experienced by $30-40 \%$ of cancer patients in acute care settings experience (Mitchell, Lancet 2011), and 10.3-14.9\% of individuals with cancer experience anxiety and depression, respectively (Holland et al, 2013; Jacobsen \& Andrykowski, 2015). These rates are critically important, as distress has been linked to cancer therapy nonadherence, increased difficulty in treatment decision making, poorer quality of life (QOL), poorer adherence to cancer surveillance screening recommendations, and poorer health behaviors (Holland et al, 2013). However, most cancer research related to psychological distress has been conducted irrespective to number of cancer diagnoses.

There is a robust body of literature documenting associations between stress and negative health outcomes (Cohen, Tyrrell, \& Smith, 1991; Kiecolt-Glaser, Marucha, Malarkey, Mercado, \& Glaser, 1995; Matthews \& Gump, 2002; Pace, Hu, \& Miller, 2007; Pyykkönen et al., 2010; Sandberg et al., 2000). In cancer specifically, distress is known to be associated with cancer diagnosis, treatment, and persistent symptoms (Holland et al., 2013; Jacobsen \& Andrykowski, 2015; Mitchell et al., 2011; National Comprehensive Cancer Network, 2015). We posit that negative psychobehavioral and biologic responses may be more severe in those with MPC, influencing the negative outcomes previously described in the literature. Importantly, findings are indicative of chronic illness in MPC survivors, and prolonged stress has been shown to decrease immune cell sensitivity to cortisol and increase pro-inflammatory cytokines and susceptibility to poor health outcomes (Cohen et al., 1995; Cohen, n.d.; Miller et al., 2002; O'Connor et al., 2009). The importance of biological response pathways is recognized and will be a source of inquiry for future research studies. 
Self-management was first described as the work of living with a chronic illness (e.g., medical management, behavioral management, and emotional management) (Corbin \& Strauss, 1988). MPC survivors live with cancer as a chronic illness (Belcher et al., 2017; Gotay et al., 2007; Thong et al., 2013). As cancer conceptualization has shifted from acute to chronic disease management, responsibility for day-to-day management has been gradually shifting from providers to individuals (Barlow, Wright, Sheasby, Turner, \& Hainsworth, 2002; Green McDonald, O'Connell, \& Lutgendorf, 2013; Klein et al., 2014; McCorkle et al., 2011). Thus, it is becoming ever more critical to determine trends in self-management, determine associations between self-management and other key variables, and eventually optimize individuals' ability to effectively manage health in the MPC survivor population.

No prior work has examined how MPC survivors self-manage their health. The proposed study directly addresses a National Institute of Nursing Research (NINR) priority to advance self-management science and dissemination of results for clinical translation (Grady \& Gough, 2014). By assessing self-management behaviors, incorporating recommended common data elements for self-management research, and evaluating associations between selfmanagement and health outcomes in MPC survivors, the proposed study will address public health and clinical priorities of self-management in chronic disease as identified by the Department of Health and Human Services, the National Academy of Medicine, the NINR, Oncology Nursing Society, and the American Cancer Society (Grady \& Gough, 2014; Knobf et al., 2015; National Cancer Survivorship Resource Center, n.d.). Additionally, tailored selfmanagement interventions have been shown to provide positive benefit in varying ethnic, geographic, and age groups, making this research promising in its applicability to diverse populations of MPC survivors (Grady \& Gough, 2014).

The proposed research study, the first step in a planned program of research, is critically important to advance the state of the science in the growing population of vulnerable survivors of MPC. The proposed study will contribute to the field of cancer survivorship research by being 
the first to identify associations among key pathways that lead to health outcomes and by identifying the behaviors MPC survivors use to manage their health. Findings from this study will assist clinicians and researchers in identifying MPC survivors at greatest risk for poor outcomes and will identify modifiable targets for future intervention development. Timely, targeted selfmanagement interventions guided by the findings from this study could reduce long-term health problems experienced by this population. Future studies will also examine biological pathways to further elucidate mechanisms resulting in poor health outcomes. The long-term research goal is to create an innovative program of research focused on the development of strategic selfmanagement interventions to interrupt stress response pathways and optimize wellness in survivors of MPC.

\subsubsection{Significance}

The proposed study is significant and timely because it will address gaps in MPC literature and add to the currently limited knowledge regarding risk factors for poor health outcomes in the rapidly expanding MPC survivor population. Additionally, the healthcare system is requiring increasing levels of disease self-management, making the previously undescribed selfmanagement variable key in understanding psychobehavioral mechanisms in the MPC survivor population. Clinically, this research has the potential to guide early identification of MPC survivors most vulnerable to prolonged stress and the associated cascade of negative health outcomes, providing a potential health promotion opportunity early on in a person's illness trajectory. Identified risk and protective factors for MPC survivors can then be used by researchers to as behavioral targets to pave the way for future, targeted intervention work.

\subsubsection{Innovation}

We argue that both the current conceptualization of cancer survivorship and practices that traditionally do not include the potential impact of MPC (e.g., often excluding MPC survivors from clinical trials), is doing a disservice to MPC survivors and has resulted in a dearth of knowledge that impacts over one million people in the United States. 
Previous MPC studies have lacked theoretical underpinnings to guide their research. This study is innovative because it will be the first study to employ a biobehavioral stress response framework and the first to begin to describe mechanisms underlying poor health outcomes in adult MPC survivors. This cross-sectional study will establish the groundwork for future longitudinal studies using this framework to investigate novel 1) biological pathways resulting in poor health outcomes and 2) temporal associations among variables. We will be the first to recruit a sample of MPC survivors to identify clinical, personal, and sociodemographic characteristics associated with perceived stress, paving the way for early identification of at-risk patients and potential targets for future interventions. Assessing self-management behaviors among MPC survivors is novel and provides information on whether interventions to promote self-management could be a potential avenue for optimizing health among MPC survivors.

\subsection{PRELIMINARY STUDIES}

\subsubsection{State of the science (manuscript \#1 in 3.1)}

The first major step in establishing the state of the science for MPC survivors included a review and synthesis of the literature to determine the relationship between MPC diagnoses and psychological distress in adult cancer survivors (S.M. Belcher et al., 2016). We hypothesized that, compared to single cancer survivors, persons with MPC diagnoses would report higher psychological distress. Effect size (ES) values were calculated using Cohen's $d$. Across the five studies included in this study, we found that MPC survivors, when compared with single cancer survivors, had lower global QOL $(d=0.32-0.37)$, poorer emotional role function and stress $(d=$ $0.08-0.20)$, greater and more frequent distress $(d=0.11-0.37)$, and greater anxiety symptoms $(d=0.15)$. Differences between MPC survivors and single cancer survivors were more variable for depressive symptoms $(d=0.01-0.22)$, and no statistically significant differences between MPC and single cancer groups were identified for sleep or suicidal ideation. Supporting our original hypothesis, effect sizes reflect small but potentially significant higher psychological distress in survivors of MPC compared with survivors of a single cancer. 


\subsubsection{Expansion of conceptual model}

As the proposed conceptual model expanded (Figure 1), subsequent MPC literature searches included the addition of the following key model concepts: perceived stress; behavioral responses, health behaviors, self-management, risk behaviors; physical health outcomes; financial toxicity; social health/social role function. Only one additional article (Dowling et al., 2013) was identified. In this study, MPC was found to be a consistent predictor of disease burden, including physical function limitations and lost productivity.

1.3.2.1 Financial toxicity. We used WRITE Symptoms ${ }^{\circledR}$ data to conduct an exploratory analysis to compare measures of QOL, including well-being, social support, and financial vulnerability, between women with recurrent ovarian cancer with and without more than one additional cancer diagnosis (Belcher et al., 2015).

Methods. Design: A secondary analysis of data from a completed three-arm randomized controlled trial (RCT) of a Web-based symptom management study of women with recurrent ovarian cancer. Sample: Participants $(\mathrm{N}=497)$ were women $\geq 18$ years old with a diagnosis of recurrent ovarian, fallopian tube, or primary peritoneal cancer, experiencing $\geq 3$ symptoms associated with cancer or cancer treatment. Setting: Participants were recruited from 53 GOG sites across the country. Within 28 days of signing informed consent, participants completed baseline questionnaires via the web-based WRITE Symptoms Questionnaire System.

Measures: Multiple dimensions of QOL were assessed using the FACT-O (well-being), ISEL-12 (social support), and sociodemographic survey (financial vulnerability, as measured by a single item asking women to rate difficulty paying for basic needs). A single item from the Charlson Comorbidity self-report was used to identify women who had been diagnosed with a second cancer (other than ovarian) within the past 3 years. Analysis: t-tests and chi-square analyses were used to compare QOL between groups.

Results. Sixty-four women (12.9\%) reported a cancer diagnosis in addition to ovarian cancer. Well-being subscales and social support did not significantly differ between the two 
groups ( $p=.15-.85$ and $p=.39$, respectively). However, a higher proportion of women diagnosed with a second cancer (51\%) reported more difficulty paying for basic needs than did those who had not been diagnosed with a second cancer (36\%), $p=.02$. Additionally, women with second cancers were more likely to report incomes $<\$ 60,000$ per year $(68 \%$ vs. $50 \%, p=.01)$.

Implications of findings to the proposed dissertation study. This preliminary, exploratory study led to further exploration of financial vulnerability and toxicity in the literature, ultimately resulting in inclusion of measures of financial toxicity in the proposed conceptual model.

\subsubsection{Conceptualization of key model concepts (manuscript \#2 in Appendix F)}

We conducted a study to describe relationships among key model concepts (see Manuscript 1 in Appendix E), specifically evaluating whether survivorship of multiple primary cancers (MPC) is associated with psychological distress, health behaviors, and benefit finding (Belcher et al., 2016).

Methods. Design: Secondary analysis of the 2010 LIVESTRONG cross-sectional survey. Sample: 238 MPC and 3,295 single cancer survivors. Setting: Online survey. Main

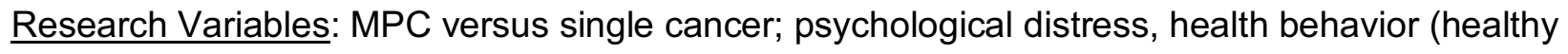
lifestyle and positive healthcare utilization), and benefit finding scores. Analyses: Chi-square tests of independence and t-tests for comparisons between persons with MPC versus single cancers. Multivariate linear and logistical regression models, adjusted for covariates, were conducted to determine associations between variables.

Results. Survivors of MPC, compared to single cancer survivors, were significantly older $(p<.001)$, less likely to have a spouse/partner $(p=.03)$, further out from original cancer diagnosis $(p<.001)$, less likely to be employed full-time $(p<.001)$, and differed by type of cancer diagnoses $(p<.001)$ and cancer survivorship stage $(p<.001)$. MPC was associated with significantly higher psychological distress $(p=.021)$ and healthcare utilization $(p=.003)$ but not healthy lifestyle $(p=.914)$ or benefit finding $(p=.263)$. 
Conclusions. Relative to those with single cancers, MPC survivors in this sample reported higher psychological distress and were more likely to receive recommended cancer screenings. Additional research is needed to understand mechanisms of psychological distress in MPC survivors. Implications for Nursing: Targeted distress screening in MPC survivors may allow for early identification and interventions to ameliorate distress and reduce negative downstream health effects. Knowledge Translation: Nurses should assess for previous cancer histories and recognize that survivorship experiences may differ between MPC and single cancer survivors. MPC survivors have increased psychological distress risk and may have needs related to living with cancer as a chronic illness. Further study of psychological distress mechanisms in MPC survivors is warranted.

Implications of findings to the proposed dissertation study. This descriptive secondary analysis reaffirmed findings of psychological distress for MPC survivors in a large, mixed-cancer survivor population and allowed for clearer conceptualization of the MPC population and key model concepts, including MPC as a chronic illness.

\subsubsection{Methodological influences}

Additional collaborative analyses have been conducted to inform this study including exploration of the influence of individual characteristics on key model concepts (Belcher, Sereika, Mattos, Hagan, \& Donovan, 2017), relationships between psychological responses and physical health (Sherwood et al., 2016), and decision making related to mode of survey administration (Hagan, Belcher, \& Donovan, 2017). 


\subsubsection{MPC science}

1.3.5.1 Summary of literature. Across studies, when compared to single cancer survivors, MPC survivors report poorer health outcomes including: higher levels of psychological distress (general and cancer-specific) (Andrykowski, 2012; Belcher et al., 2017; Belcher et al., 2016; Burris \& Andrykowski, 2011; Gotay et al., 2007; Thong et al., 2013); more risky health behaviors including physical inactivity, smoking, and alcohol use (Burris \& Andrykowski, 2011); increased symptom burden including diarrhea, fatigue, and pain (Thong et al., 2013); poorer physical function (Andrykowski, 2012; Burris \& Andrykowski, 2011; Dowling et al., 2013; Gotay et al., 2007; Thong et al., 2013); more financial hardship and lost productivity (Belcher et al., 2015; Dowling et al., 2013); higher numbers of comorbidities (Andrykowski, 2012; Gotay et al., 2007; Thong et al., 2013); and interference with social activities (Thong et al., 2013).

1.3.5.2 Gaps in the science and future directions. While previous studies add to the science by including samples from various geographical regions representing a broad range of cancer diagnoses, this study will address the following critical gaps to advance MPC science:

1. Atheoretical. No studies to date have identified theoretical models to guide scientific inquiry in this area, leading to a lack of conceptual clarity for scientific advancement.

2. Study design. Previous MPC research has been devoted to comparing groups of cancer survivors based upon the number of cancer diagnoses (e.g., no cancer controls versus 1 primary cancer versus $\geq 2$ primary cancer diagnoses). Differences among groups have now been established, and the time is right to look within MPC survivor groups to identify a) the factors that put particular MPC survivors at risk for poor health outcomes and b) potential mechanisms that link exposure to the chronic stresses of MPC with negative health outcomes.

3. Negative behavioral responses. While one previous study evaluated dietary intake and physical activity in this patient population (Burris \& Andrykowski, 2011), no studies have formally evaluated positive behavioral responses (self-management) among survivors of MPC. Positive self-management behaviors are important to understand in this population, as they 
have potential to interrupt negative pathways and restore optimal wellness in the context of chronic illness, such as occurs with MPC diagnosis.

4. Inconsistent use of valid and reliable measures. We will avoid measurement pitfalls and limitations of previous MPC studies by using valid and reliable instruments (Redeker et al., 2015).

5. Reliable clinical data. Previous overreliance on self-reported clinical data will be avoided by incorporating cancer registry disease classifications and confirming MPC diagnoses via systematic medical record review using standard definitions of MPC (Begg, 1999; Johnson et al., 2007; Koubkova, Hrstka, Dobes, Vojtesek, \& Vyzula, 2014).

6. Variable inclusion criteria. Previous study samples differed by inclusion criteria, particularly by age at diagnosis and by cancer type. We will address this gap by removing potential developmental influences on outcomes by including only survivors of cancers diagnosed during adulthood and by excluding noninvasive skin cancer cases, which have been shown to not differ from general noncancer populations in regard to psychosocial issues (Holfeld, Hogan, Eldemire, \& Lane, 1990).

7. Diversity. Previous studies have largely lacked racial and ethnic diversity. While recognizing the limitations of the planned recruitment frame for diversity of race and ethnicity, processes will be put in place to oversample for minority groups when possible.

\subsection{RESEARCH DESIGN AND METHODS}

\subsubsection{Design}

We will implement a cross-sectional design using a self-administered, mixed-mode survey of valid and reliable patient reported outcomes with survivors of two primary cancer diagnoses, or multiple primary cancers. A cross-sectional survey design is feasible and will allow for testing of associations/correlations among variables in the theoretical model (Fowler, 2014; Groves et al., 2009). Identified patterns of association will be tested for temporal ordering in future longitudinal study. 


\subsubsection{Sample}

1.4.2.1 Sampling design. Stratified systematic sampling with oversampling for minorities will be used to select our study sample.

1.4.2.2 Sampling frame and elements. The UPMC Cancer Network Registry (1990 - current) will serve as our sampling frame. The sampling unit will be the registry participant ID number, and MPC survivors will be the sampling elements.

1.4.2.3 Inclusion criteria. 1) history of two or more primary cancers, as defined by Surveillance, Epidemiology, and End Results (SEER) Program coding rules (Johnson et al., 2007), both diagnosed in adulthood [ $\geq 18$ years old]; 2) first diagnosis is consistent with one of the 7 most prevalent first cancers experienced by male/female MPC survivors (American Cancer Society, 2009), including: breast, prostate, colorectal, urinary bladder, uterine, melanoma, kidney/renal pelvis, lung/bronchus, oral cavity/pharynx, thyroid, and ovary (see Figure 2); 3) stage I-III cancers; 4) between 1 and 10 years following active treatment/no evidence of disease; and 5) able to read and complete questionnaires in English.

\subsubsection{Exclusion criteria. 1) non-melanoma skin}

cancers; 2) in situ cancers; 3) stage IV cancers; and 4) history of recurrence.

\subsubsection{Sampling plan. Gotay and colleagues have} been the only research team to our knowledge to specifically recruit a sample of adults with MPC from a

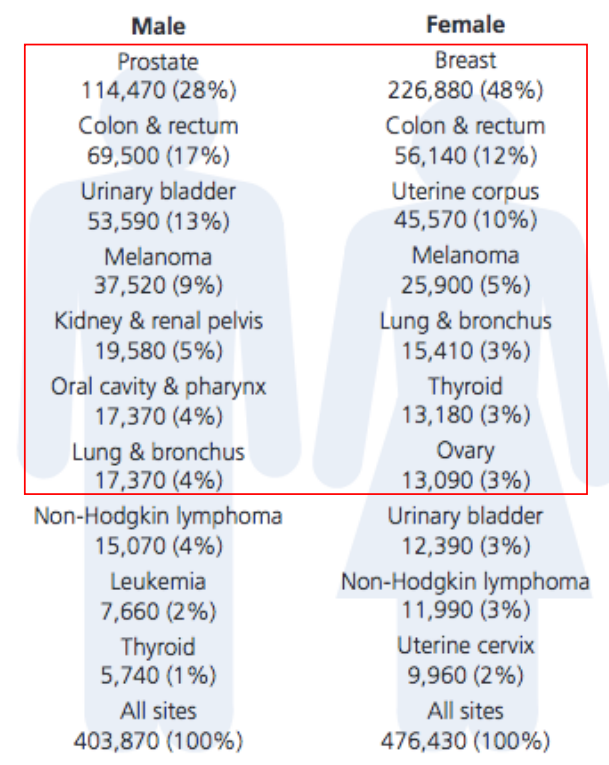

Figure 2. Cancer sites of study inclusion. Estimated survivors who have been diagnosed with more than one cancer, by site of first primary. Diagnoses in red box indicate top 7 cancers for men and women which will be included in this study. Image: (American Cancer Society, 2009). state tumor registry (Gotay et al., 2007). They reported the following recruitment results: 1) $27.7 \%$ were unable to be contacted due to out of date contact information or being deceased, 2) physicians requested that $1.2 \%$ of potential participants not be contacted, and 3) $56 \%$ of those 
contacted via mail returned completed surveys. Balancing cost, feasibility, and analytic power, we plan to send initial invitations to 1,440 MPC survivors (see detailed sampling plan below). Based on conservative estimates from the Gotay study, we expect that we will be able to reach approximately $60 \%$ of potential MPC participants, leaving approximately 864 potential responders. Targeting rates of participation similar to those in the Gotay study (approximately $50 \%$ ), we would have an estimated 432 respondents in this proposed study (rounded up to 440 to allow for equal recruitment attempts among groups of cancer diagnosis sites).

\subsubsection{Recruitment and data collection procedures}

Cancer Registry personnel will assist in identifying potential study participants from the Cancer or Cancer-like Blood Disorder Registry (UPCI protocol \#03-038). Personnel from the UMPC Network Cancer Registry will first match registry participants meeting the study's criteria to the UPCI protocol 03-038 patients. For living patients in common between these data sets, our research team will be provided with the names and mailing addresses for the matching patients in order to conduct the recruitment mailings ourselves. All MPC survivors in the registry meeting inclusion criteria will be considered in the sampling frame. Based on July 2016 Registry data, we expect that all non-white and Hispanic MPC survivors $(n=323)$ will be invited to participate. The remaining 1,117 potential white participants will be randomly sampled from the larger sampling frame. UPMC physician champions who are content experts in the cancers being targeted are being enlisted to introduce the study to prospective participants via a standardized invitation letter facilitated by the study team in collaboration with the Cancer Registry. In response to the invitation, individuals can select their mode of survey delivery (pencil/paper or online) by returning an enclosed postage-paid card, calling the study line, or

emailing the PI. Participant follow-up contacts will occur up to four times over a 6-week period based on Dillman's Tailored Design Method (see study flow chart in Figure 3). Additional participant contact details are provided in the Research Participant Risk and Protection human subjects section of this document. 


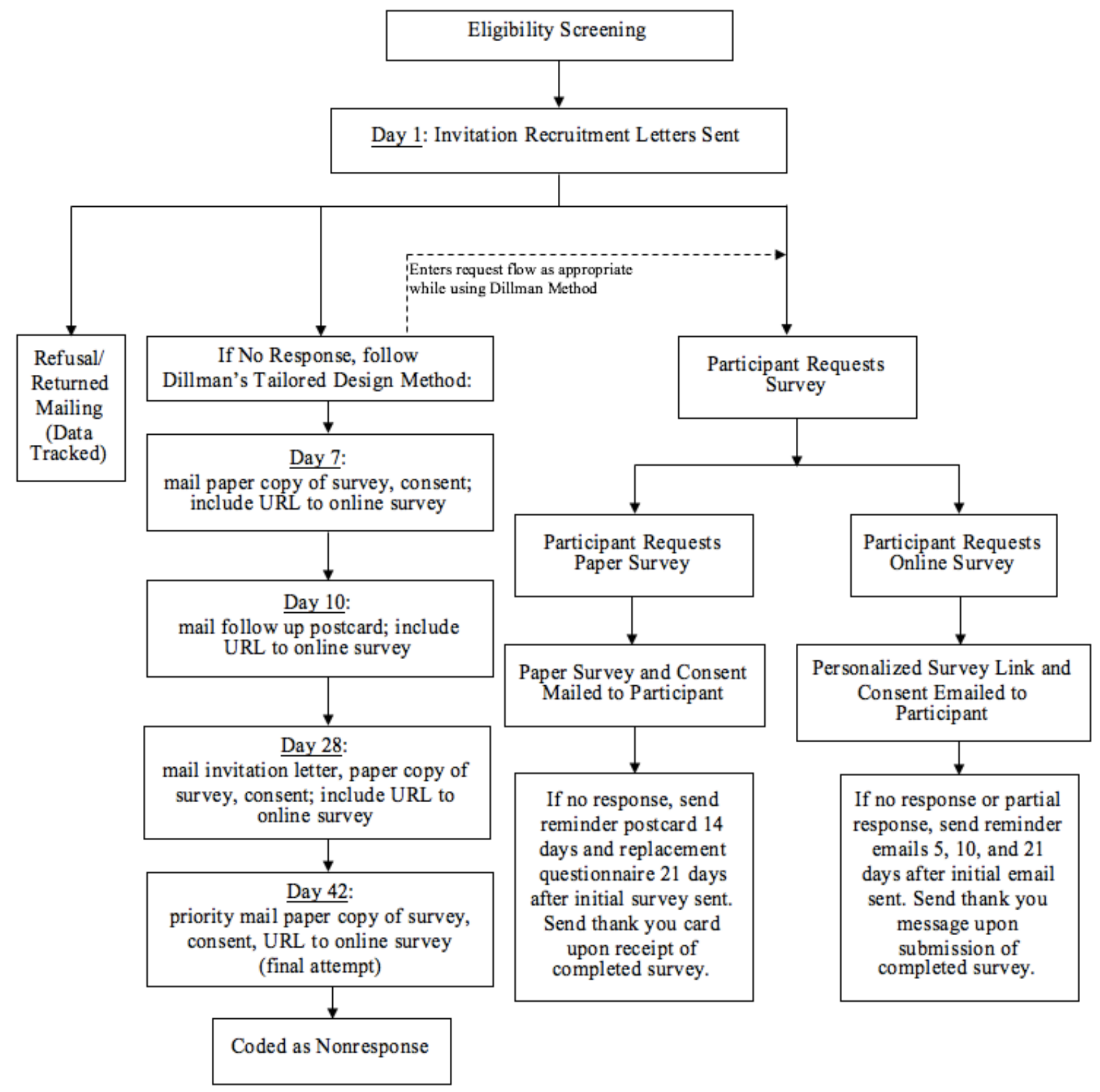

Figure 3. Study flow chart. This figure illustrates the planned study flow. Study protocol allows for $+/-3$ days adjustment to timeline to conduct study procedures.

1.4.3.1 Dillman's Tailored Design Method. Dillman's Tailored Design Method (Dillman, Smyth, \& Christian, 2014) will be followed to maximize recruitment and response. This method, based on social exchange theory, embeds processes and procedures to increase survey requests and 
motivation to complete the survey. This is achieved by establishing trust, increasing perceived benefits of survey completion, and decreasing expected cost of participation. We will establish trust in the following ways: 1) UPMC disease-based specialists will introduce and provide written endorsement of the study; 2) stressing the importance of the knowledge to be gained about MPC survivors in this study; and 3) ensuring participants' privacy. By providing study background in the survey, demonstrating respect for individuals, compensating participants with a $\$ 5$ gift card in recognition of participants' time and expertise as survivors, and providing social validation of the persistent cancer-related influences experienced by MPC survivors, we will increase participants' perceived benefit of survey completion. Decreasing expected costs to participants will be achieved in the following ways: allowing convenient participation, only including necessary measures and using sort forms when possible, minimizing the amount of private information requested, and avoiding authoritative language.

1.4.3.2 Data collection. Mixed-mode data collection was chosen for the proposed study in order to maximize response rates and data quality (de Leeuw \& Berzelak, 2016; Dillman \& Edwards, 2016). Surveys will be administered both via Computer Assisted Self-Interviewing via Qualtrics (University of Pittsburgh Computing Services and Systems Development, n.d.) and pencil/paper, based on respondent preference. Participants will complete a one-time battery of patient reported outcomes that will include valid measures of key model concepts (see Table 1). To ensure accuracy (Abraham et al., 2009; Bergmann et al., 1998; Bergmann, Byers, Freedman, \& Mokdad, 1998; Inoue et al., 2011; Yoshinaga, Sasaki, \& Tsugane, 2001) and to decrease participant burden, clinical data will be extracted from the medical record using a standardized data extraction form. All data collected via pencil/paper mode and medical record extraction will be entered into a separate Qualtrics-based electronic database by the study PI and undergraduate student research assistant personnel via direct data entry, allowing for simple data merging into the final comprehensive dataset. Quality assurance for direct data entry variables (Agency for Healthcare Research and Quality, 2014) will be ensured by using 
built in Qualtrics validation features (e.g., limiting characters and ranges allowed for entered variables, such as only allowing 5 characters for zip code variables) and conducting accuracy audits of every $10^{\text {th }}$ paper survey entered into the database; if errors are uncovered, audit frequency will be increased. Additionally, the PI is currently exploring options for collecting and banking biological samples for future analyses.

\subsubsection{Measures}

A paper copy of the survey battery administered to study participants is included in Appendix A. The online version of the survey was designed to closely mirror the paper copy format.

Variables, levels of measurement, measures, and psychometric properties are presented in Table 1. 
Table 1.

Dissertation Study Concepts, Measures, Levels of Measurement, and Psychometric Properties

\begin{tabular}{lll}
\hline \multicolumn{1}{c}{ Concept } & Level & Self-Report Measures, Description, and Psychometric Properties \\
\hline Perceived & & \multicolumn{1}{c}{ Perceived Stress } \\
Stress, Global & Highly Ordinal $^{*}$ & $\begin{array}{l}\text { The Perceived Stress Scale (PSS) is a 10-item measure of globally perceived } \\
\text { stress that uses 5-point Likert scale items. Higher scores (0-40) indicate more } \\
\text { stress. Extensive reliability and validity (concurrent and predictive) (Cohen, } \\
\text { Kamarck, \& Mermelstein, 1983) has been demonstrated and confirmed in various } \\
\text { populations. Cronbach's } \alpha \text { ranges from .86 to .92 in women following breast cancer } \\
\text { surgery and demonstrated a two-factor solution of positive versus stress items with } \\
\text { stability over 12-month intervals (Golden-Kreutz, Browne, Frierson, \& Andersen, }\end{array}$ \\
& 2004).
\end{tabular}

Perceived

Stress, Cancer-

Specific

Perceived

Stress, Cancer-

Worry

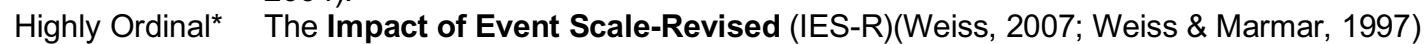

is 22-item Likert-scale measure of event-specific post-traumatic stress adapted from Hurowitz and colleagues' earlier Impact of Event Scale. (Weiss \& Marmar, 1997) The IES-R includes subscales for intrusion, avoidance, and hyperarousal (verified by factor analyses; correlated with HADS subscales). It has been used specifically to assess how distressed patients have felt in the past 7 days about a range of cancer-related difficulties and demonstrated extensive reliability and validity in various cancer patient populations, including post-treatment survivors (Cronbach's $\alpha$ $=.77$ - .96; 3-day test-retest reliability $r=.97$ ) (Salsman, Schalet, Andrykowski, \& Cella, 2015; Vodermaier, Linden, \& Siu, 2009).

Highly Ordinal $^{*} \quad$ The Assessment of Survivor Concerns (ASC) (Gotay \& Pagano, 2007; Hershman et al., 2013; Thewes et al., 2012) is a 5-item Likert-scale instrument to measure fears about cancer recurrence and health in mixed population cancer survivors. The item responses range from 1 (not at all) to 4 (very much), with higher scores indicating higher worry. The instrument was developed based on work with previous cancer survivors and has undergone extensive validation work with mixed cancer survivor populations. The 3-item cancer-specific worry subscale to be used in this study has demonstrated high internal consistency (Cronbach's $\alpha=.93$ ); it measures worry about cancer recurrence, new cancer diagnoses, and future diagnostic testing.

\section{Psychological Responses}

Depressive

Symptomatol-

ogy

Highly Ordinal

The Personal Health Questionnaire Depression Scale (PHQ-8), (Kroenke, Spitzer, \& Williams, 2001; Kroenke et al., 2009; Ory et al., 2013) an adaptation of the valid and reliable PHQ-9 depression scale with omission of the suicidal ideation item, is an 8-item clinically validated measure of depressive symptoms. The PHQ-8 is preferred over PHQ-9 when measures are self-administered. Participants are asked how often over the last 2 weeks they have been bothered by different problems. Higher scores (0-24) indicate more severe depression. Cronbach's $\alpha$ ranges from .80 to .86 in mixed cancer populations and individuals with chronic disorders. To decrease participant burden, individuals who score 0 on the first two items of this measure will be instructed to skip the remaining items in the measures, as the PHQ-2, which taps into depressed mood and anhedonia, has been shown to be an effective screen for depression in validity and sensitivity studies (American Psychlogical Association, 2017; Kurt Kroenke, Spitzer, \& Williams, 2003).

Anxiety Highly Ordinal*

The Generalized Anxiety Disorder 7-item scale (GAD-7) (Kroenke, Spitzer, Williams, Monahan, \& Lowe, 2007; Löwe et al., 2008; Spitzer, Kroenke, Williams, \& Lo, 2006) is a clinically validated measure of generalized anxiety. Participants are asked how often over the last 2 weeks they have been bothered by a list of problems. A cut point of 10 was established to optimize sensitivity and specificity ( $89 \%$ and $82 \%$, respectively), with higher scores indicative of greater anxiety. Confirmatory factor analyses confirmed a 1-factor structure and equitability for gender and age for use in the general population to detect various types of anxiety (Cronbach's $\alpha=.89-.92$; intra-class correlation [ICC] = .83 for test-retest reliability). Criterion validity was also established. To decrease participant burden, individuals who score 0 on the first two items of this measure will be instructed to skip the remaining items in the measure, as the GAD-2 is recommended as an "ultra short" diagnostic instrument for generalized anxiety disorder. A positive GAD-2 result is a score $\geq 3$, while a positive GAD-7 result is a score $\geq 8$. 
Table 1 (continued).

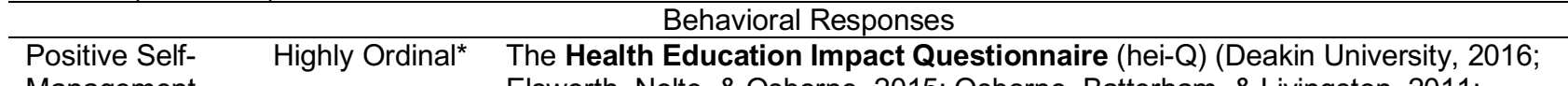

Management

Elsworth, Nolte, \& Osborne, 2015; Osborne, Batterham, \& Livingston, 2011; Osborne, Elsworth, \& Whitfield, 2007) is a 40-item scale used internationally to assess 8 domains (factor structure validated) of self-management in chronic illness, including cancer: health directed activities (Cronbach's $\alpha=.80$ ); positive and active engagement in life (Cronbach's $\alpha=.86$ ); emotional distress (Cronbach's $\alpha=.89$ ); self-monitoring and insight (Cronbach's $\alpha=.70)$; constructive attitudes and approaches (Cronbach's $\alpha=.81$ ); skill and technique acquisition (Cronbach's $\alpha=$ .81 ); social integration and support (Cronbach's $\alpha=.86$ ); and health service navigation (Cronbach's $\alpha=.82$ ). Internal consistency ( $\geq .8)$ and discriminant validity have been established across sex, age, education, and ethnic background groups.

Risky

Behaviors:

Highly Ordinal*

$\dagger$ The PROMIS Alcohol Use Short Form 7a (Gibbons et al., 2016; Patient-Reported

Alcohol Use Outcomes Measurement Information System, 2014; Pilkonis et al., 2016) assesses drinking patterns, cue-based drinking, cravings to drink, and efforts to control drinking indicative of problematic drinking at the moderate and low severity levels for adults with chronic illness. Participants are first asked if they have consumed any alcohol in the previous 30 days and, if yes, respond to 7 items scored on a 5-point Likert scale, with higher scores indicating more risky alcohol use behavior.

Risky Highly Ordinal*

Behaviors: The Cancer Patient Tobacco Use Questionnaire (C-TUQ),(Land et al., 2016; Land et al., 2016; National Cancer Institute, 2016) developed by joint National Tobacco Use by Cancer Patients Cancer Institute and the American Association for Cancer Research task force, was created to assess tobacco use in cancer patients and survivors. The instrument includes both 4 core constructions and extension items to assess domains of smoking history and current use, use relative to cancer diagnosis and treatment, other tobacco product use, cessation, and second-hand exposure. Expert review panels and iterative cognitive interviews were conducted during instrument development.

Health Outcomes: Global Health, Physical Health, Financial Toxicities, Social Role Function

\begin{tabular}{|c|c|c|}
\hline \multicolumn{3}{|r|}{ Global Health } \\
\hline Global Health & Highly Ordinal* & $\begin{array}{l}\dagger \text { The PROMIS SF v1.1 - Global Health, is a } 10 \text {-item measure intended to } \\
\text { measure general, self-reported health. It includes } 10 \text { global health items from the } 5 \\
\text { core PROMIS domains and has been tested in broad clinical samples, including } \\
\text { adults with cancer. Previous research (Hays et al., 2009) has demonstrated } \\
\text { construct validity of items, and found two underlying domains reflected during factor } \\
\text { analysis: mental and physical health (Cronbach's } \alpha=.81 \text { and } .86 \text {, respectively). } \\
\text { Items are scored on a 5-point Likert scale with higher scores indicating better global } \\
\text { health. } \\
\text { This instrument is recommended by NINR as a common data element for self- } \\
\text { management research (Moore et al., 2016) and will be analyzed for descriptive } \\
\text { purposes, not as part of measurement model. }\end{array}$ \\
\hline
\end{tabular}

Physical

Function

Treatment-

Related

Symptoms and

Interference
Physical Health

Highly Ordinal $^{*}$

$\dagger$ The PROMIS Physical Function Short Form 20a (Patient-Reported Outcomes Measurement Information System, 2015; Rose et al., 2014; Schalet et al., 2016) measures capability rather than actual performance of physical activities, including dexterity, walking or mobility, neck/back, and instrumental activities of daily living. PROMIS recommends using the 20 a short form if variability is expected and various subdomain information is desired, thus the 20 a was selected for this study. Higher scores indicate better physical function. Validity over time was established in adults with chronic illness, including cancer. Field testing and criterion testing was consistent, irrespective of health, age, and disease group/number of chronic conditions.

Highly Ordinal* The MD Anderson Symptom Inventory (MDASI) (Aktas, Walsh, \& Kirkova, 2015; Cleeland et al., 2000; Cleeland, 2016) is a multi-symptom patient-reported outcome measure for clinical and research use. It is used to both assess symptom severity and interference with daily living. The 21-item core bank of symptoms consists of those symptoms that are most frequent and/or severe in cancer patients and yielded 2 symptom factors and interference scales (Cronbach's $\alpha=.85-.87 ; .82-.87$; and $.91-.94$, respectively). The core accounted for $64 \%$ of symptom distress variance in principal factor analysis. Sensitivity to disease severity performance status (ECOG) and treatment status was confirmed. 
Table 1 (continued).

\begin{tabular}{|c|c|c|}
\hline Comorbidity & Ratio & $\begin{array}{l}\text { The Charlson Comorbidity Index }(\mathrm{CCl}) \text { was originally developed in } 1987 \\
\text { (Charlson, Pompei, Ales, \& MacKenzie, 1987) as a weighted sum measure of } \\
\text { chronic disease burden used to predict long term prognosis and outcomes and } \\
\text { remains a population instrument for comorbidity risk adjustment. The brief } 10 \text {-item } \\
\text { self-report form selected for this study (short form) has been demonstrated to } \\
\text { predict 1-year mortality in racially diverse populations and performed well compared } \\
\text { to ICD-9 generated CCI scores (Chaudhry, Jin, \& Meltzer, 2005). }\end{array}$ \\
\hline \multicolumn{3}{|r|}{ Financial Toxicity } \\
\hline $\begin{array}{l}\text { Financial } \\
\text { Toxicity }\end{array}$ & Highly Ordinal* $^{*}$ & $\begin{array}{l}\text { The 11-item COmprehensive Score for financial Toxicity (COST-FACIT, version } \\
\text { 1) tool (De Souza et al., 2016; De Souza et al., 2014) was developed using a step- } \\
\text { wise approach to provide a patient reported outcome measure of financial toxicity } \\
\text { (1-factor latent variable) in patients with cancer. Item responses are scored on a } \\
\text { Likert scale of not at all to very much }(0-4) \text {, with lower scores indicating worse } \\
\text { financial toxicity. Internal consistency (Cronbach's } \alpha=.92) \text {, test-retest reliability } \\
(\mathrm{ICC}=.80,95 \% \mathrm{CI}=.57-.92) \text {, convergent validity (POMS Pearson correlation }= \\
.26, p<.001 ; \text { household income Pearson correlation }=.28, \mathrm{P}<.001) \text {, divergent validity } \\
\text { (Marlowe-Crowne Social Desirability Scale Pearson correlation }=.11, \mathrm{p}=.11) \text {, and } \\
\text { correlation with health-related quality of life (FACT-G Pearson correlation }=.42 \text {, } \\
\text { p<.001; EORTC-QOL Pearson correlation }=.33, \mathrm{P}<.001) \text { have been validated in } \\
\text { samples of adults with advanced cancer. }\end{array}$ \\
\hline $\begin{array}{l}\text { Economic } \\
\text { Hardship }\end{array}$ & Highly Ordinal* & $\begin{array}{l}\text { The Economic Hardship questionnaire (Barrera, Caples, \& Tein, 2001), a 17-item } \\
\text { measure of perceived economic hardship, is based off of four measures of } \\
\text { subjective hardship: financial strain (Vinokur, Price, \& Caplan, 1996); inability to } \\
\text { make ends meet (Conger \& Elder Jr., 1994; Pearlin, Menaghan, Lieberman, \& } \\
\text { Mullan, 1981); not enough money for necessities (Conger \& Elder Jr., 1994; Pearlin } \\
\text { et al., 1981); and economic adjustments/cutbacks (Conger \& Elder Jr., 1994). This } \\
\text { scale has demonstrated factor structure, internal structure congruence, and validity } \\
\text { in urban families across gender, language (English and Spanish), and ethnicities } \\
\text { (African American, European American, and Mexican American), in } 4 \text { different } \\
\text { domains: financial strain; inability to make ends meet; not enough money for } \\
\text { necessities; and economic cutbacks and adjustments (Cronbach's } \alpha=.70-.85 \text { ). } \\
\text { The first } 3 \text { domain items are rated on a 5-point Likert-type scale and mean subscale } \\
\text { scores are created. Economic Adjustments and Cutbacks are assessed with } 9 \\
\text { items, such as added another job, received government assistance, and sold } \\
\text { possessions because money was needed. Participants indicate whether these } \\
\text { events have occurred in the past month. This subscale score is the total number of } \\
\text { events that occurred (0-9). }\end{array}$ \\
\hline
\end{tabular}

Social Role Function

Social function, a part of social health with implications for impact on physical health, is broken down into ability to and satisfaction with participation.(Bode et al., 2010) Both instruments are scored on a never (5) to always (1) Likert scale.

Social Role Highly Ordinal* † The PROMIS - Ability to Participate in Social Roles and Activities Short Form

Function, Ability 8a (Bode et al., 2010; Hahn et al., 2014) is a short form intended to assess perceived ability to participate in usual social roles and activities. Higher scores represent better abilities.

Social Role

Participation,

Highly Ordinal* † The PROMIS - Satisfaction with Participation in Social Roles Short Form 8a

Satisfaction (Bode et al., 2010; Hahn et al., 2014, 2016) is a short form intended to assess perceived satisfaction with participation in usual social roles and activities. Responsiveness to change was demonstrated in a sample of adults with chronic illness.

Personal, Demographic, and Clinical Characteristics

\begin{tabular}{|c|c|c|}
\hline \multicolumn{3}{|r|}{ Personal } \\
\hline $\begin{array}{l}\text { Personality: } \\
\text { Neuroticism and } \\
\text { Conscientious- } \\
\text { ness }\end{array}$ & Highly Ordinal* & $\begin{array}{l}\text { The 50-item International Personality Item Pool (IPIP)(Goldberg, 1999; Goldberg } \\
\text { et al., 2006; Socha, Cooper, \& McCord, 2010) includes } 10 \text { items each for five } \\
\text { personality factors, including extroversion/introversion, agreeableness, } \\
\text { conscientiousness, emotional stability-neuroticism, and intellect. The present study } \\
\text { is measuring only subscales for Neuroticism (Cronbach's } \alpha=.86 \text { ) and } \\
\text { Conscientiousness (Cronbach's } \alpha=.85 \text { ), as they have previously been linked to } \\
\text { self-management. Respondents are asked to rate present behaviors on a } 1 \text { (very } \\
\text { inaccurate) to } 5 \text { (very accurate) scale. }\end{array}$ \\
\hline
\end{tabular}




\subsubsection{Data analysis plan}

As described in Table 1, individual constructs have been theoretically defined and operationalized. The proposed conceptual model (Figure 1) will guide data analyses. Collected data will be exported to a database for analyses.

1.4.5.1 Descriptive statistics. Descriptive and exploratory analyses will first be performed using IBM ${ }^{\circledR}$ SPSS ${ }^{\circledR}$ software, version 25 (IBM Corp., Armonk, NY) to identify any data anomalies (e.g., missing data or outliers that may be a result of data entry error or invalid participant responses) that might invalidate findings of the primary aim analyses to be conducted. To confirm external validity, sample characteristics will be compared to what is currently known in existing MPC literature. For continuous variables, appropriate descriptive statistics including graphical representation will be computed to describe sample characteristics and determine observed variable distributions. For categorical variables, we will examine frequency distributions to ensure adequate category size; categories will be meaningfully collapsed as possible if inadequate category sizes are observed. Pairwise correlations will be calculated to summarize bivariate associations between variables.

For the variables previously described in Table 1, we will report frequency counts and percentages for categorical variables. For the central tendency and dispersion for categorical variables, mode and range will be reported for nominal variables, and median and interquartile range (IQR) will be reported for ordinal variables. For continuous variables, we will describe central tendency as means and dispersion as standard deviations for normally distributed data and medians and IQRs or semi-quartile ranges (SQR) for non-normal data distributions.

1.4.5.2 Data screening procedures. Statistical assumptions for planned analyses will be checked for violations (e.g., independence, normality, homoscedasticity, multivariate normality, and linearity) prior to performing any primary aim analyses. Measured variables will be screened for univariate outliers by z-scores for continuous type variables with absolute values $>3.29$ and lopsided distributions (i.e., small categories < $10 \%$ ) for categorical variables suggesting 
univariate outliers. Univariate and bivariate data exploration with graphical plots will be conducted to determine outliers, potentially influential cases, and linearity of relationships among variables. Mahalanobis distance will be used to assess for multivariate outliers. Correlations (i.e., determinant of the covariance matrix); variance inflation factors (VIF); tolerance; and Belsley, Kuh, and Welch (BKW) diagnostics (Mason, 1987) will be used to assess for multicollinearity.

Data transformations or more statistically robust measures will be used in such instances of assumption violations. Robust maximum likelihood estimation methods are available and will be employed to fit all models if multivariate normality assumptions are questionable. We will also examine correlations between potential covariates/confounders and outcome variables and psychometric properties of multi-item scales. Cronbach's coefficient alpha will be used to estimate internal consistency of multi-item scales. All tests will be twotailed, with statistical significance criterion set at $p<.05$.

Because our primary multivariate analytic technique, SEM, is based on covariances, parameter estimates and $\chi^{2}$ tests of fit are sensitive to sample size and require larger samples (Tabachnick \& Fidell, 2013). We will assess for representativeness of population due to dropouts/exclusions and amount and patterns of missing data. SEM allows for data missing completely at random (MCAR) and missing at random (MAR) through full information maximum likelihood (FIML) methods (Enders \& Bandalos, 2001).

1.4.5.3 Data analysis procedures. SEM, using Mplus, version 8 (Muthén \& Muthén, 2017) will be conducted to analyze structural linear relations between latent constructs and considering the loadings of measured variables and their reliabilities in the proposed multivariate model using results from the primary data collection. The following SEM testing plan is based on standard procedures (Tabachnick \& Fidell, 2013).

Aim 1a. The first step will be to fit the full measurement model to identify any issues with measurement. The hypothesized full structural equation model is presented in Figure 4. The 
independence model will be conducted first to test whether all variables are uncorrelated (this is anticipated to be easily rejected, allowing us support to move on to the next phase). Next, we will test the hypothesized model using appropriate test statistics and fit indices. Evaluation of model fit has been described as a "gestalt process" (Little, 2013); model fit (Little, 2013) will be evaluated based on theory and 1) statistical rationale using exact, or statistical, fit [ $\chi^{2}$ statistic] and 2) modeling rationale, which incorporate a) absolute fit, or the measured distance between the hypothesized model and the perfect fitting model [root mean square error of approximation (RMSEA)] and b) relative fit, or index of the percent improvement from the null to the saturated or perfect model (comparative fit indices [CFI] and Tucker Lewis Index [TLI]). Taking statistical rationale into consideration, which are influenced by sample size, the following modeling rationale cutoffs will be considered and interpreted as acceptable when evaluating model fit in this study: 1) RMSEA and associated $90 \%$ confidence intervals are $\leq .05$ and 2) CFI $\geq .90$ (Little, 2013).

Aim 1b. Post-hoc model modifications will be conducted to attempt to improve model fit and parsimony. Model paths may be added or removed based on $\chi^{2}$ difference tests and path coefficient significance testing results. The Wald test will be used to determine if there are any parameters that could be deleted from the model without impacting model fit. In a model that fits the data well, we would anticipate that the paths should all be significant and model modification indices should be small. The final model will be presented with significant coefficients in standardized form.

Aim 2. Pending determination of model adequacy, specific aspects of the model will be tested for secondary aims. Paths will be added to explore the relationships among the clinical, personal, and sociodemographic characteristics and the latent variable of perceived stress. Models will be compared using $\chi^{2}$ difference testing (Tabachnick \& Fidell, 2013).

Exploratory Aim 3. Descriptive and exploratory analyses will be conducted to characterize self-management behaviors, as measured by the hei-Q. 
1.4.5.4 Sample size justification. Based on cost and feasibility of this dissertation study, we have budgeted for a target sample of 440 MPC survivors returning completed surveys. To fit the full structural equation model, as displayed in Figure 4, will use data from 21 measured variables yielding 21 variances and 210 covariances to estimate and test 57 parameters. As specified, with 172 degrees of freedom and a sample size of 440 , we will have $>.99$ power to fit and test for a close fit based on RMSEA at a significance level of .05.

\subsection{STUDY TIMELINE}

Table 2

Study timeline

Study activities

IRB submission and approval $\frac{7 / 17}{\mathrm{X}} \quad \underline{8 / 17-12 / 17} \quad \underline{1 / 18-2 / 18} \quad \underline{3 / 18-4 / 18} \quad \underline{5 / 18-6 / 18}$

Solidify procedures with Research

Registry and Physician Champions

Data collection

Data cleaning

Data analysis

Abstract and manuscript dissemination

Dissertation Defense

\begin{tabular}{lll}
$X$ & $X$ & $X$ \\
$X$ & $x$ & $x$ \\
& & $x$ \\
\hline
\end{tabular}




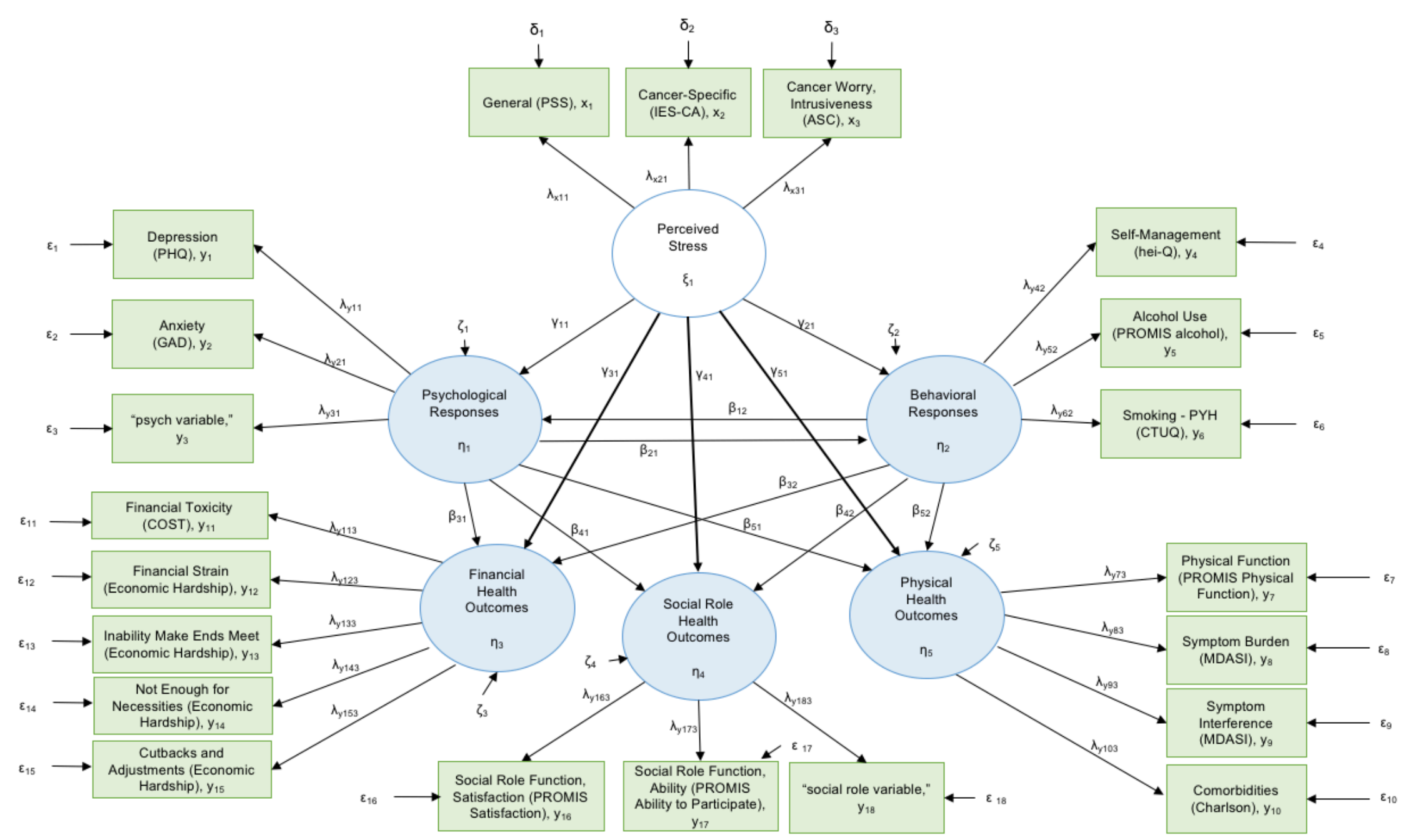

Figure 4. Full hypothesized structural equation modeling model. Blue circles = latent variables; green rectangles $=$ measured variables . Greek letter key: $\xi(\mathrm{ksi})=$ exogenous construct; $\eta$ (eta) = endogenous construct; $\beta$ (beta) = estimated regression of one endogenous construct on another endogenous construct; $\mathrm{Y}$ (gamma) = estimated regression of one exogenous construct onto an endogenous construct; $\lambda($ lambda $)=$ estimated loading of an indicator on a construct; $\delta$ (delta) = residual variance of an indicator for an exogenous construct; and $\varepsilon$ (epsilon) = residual variance of an indicator for an endogenous construct. 


\subsection{POTENTIAL LIMITATIONS AND ALTERNATIVE APPROACHES}

Limitations and pitfalls are anticipated to be encountered during the course of this research. It is possible that use of the voluntary research registry through the UPMC Network Tumor Registry will not allow us to achieve our sampling goals. If necessary to achieve recruitment goals, our next step would be to utilize the honest broker system within the larger UPMC Network Cancer Registry, facilitating mailings from physician champions under the supervision of the study principal investigator $(\mathrm{PI})$ to introduce the study to prospective participants who fall within our inclusion criteria. Methodologically, it is possible that the data will not lend itself well to SEM analyses. If models will not hold using full SEM, our alternative approach will be to conduct a path analysis or a series of regression models to determine associations among variables in the model. Additionally, it is possible that the sample of MPC survivors in the UPMC registry will not generalize well to the larger MPC population. Previous MPC literature has been largely conducted with non-minority participants with higher socioeconomic statuses. To protect against sampling bias, 1) we are offering participants two modes of survey completion, allowing for people with limited online access to still participate in this research, and 2) we will be oversampling for minority participants. Findings will be compared to existing MPC literature and will be discussed in publications.

\subsection{PUBLICATIONS RELEVANT TO THE PROPOSED RESEARCH}

\section{Refereed Articles * = Data Based}

1. * Belcher, S. M., Low, C. A., Posluszny, D. M., Schear, R., Kramer, R. E., \& Donovan, H. S. (2017). Correlates of psychological distress, health behaviors, and benefit finding in survivors of multiple primary cancers: Results from the 2010 Livestrong survey. Oncology Nursing Forum, 44 (6), 703-711. doi: 10.1188/17.ONF.703-711

2. ${ }^{*}$ Hagan, T. L., Belcher, S. M., \& Donovan, H. S. (2017). Mind the mode: Differences in paper vs. web-based survey modes among women with cancer. Journal of Pain and Symptom Management, 54 (3), 368-375. doi:10.1016/j.jpainsymman.2017.07.005

3. * Belcher, S. M., Hausmann, E. A., Cohen, S. M., Donovan, H. S., \& Schlenk, E. A. (2016). Examining the relationship between multiple primary cancers and psychological distress: A review of current literature. Psycho-Oncology, 26 (7), 2030-2039. doi:10.1002/pon.4299 


\section{Published Abstracts}

1. Nilsen, M., Belcher, S., Donovan, H., Klem, M. L., Morrison, A., Sereika, S., \& Johnson, J. (2018). Late and long-term treatment effects among survivors of head and neck cancer at least 5 years post-treatment: A systematic review. International Journal of Radiation Oncology, Biology, Physics (Red Journal), 100 (5), 1401. doi:10.1016/j.ijrobp.2017.12.242

2. Belcher, S. M., Sereika, S. M., Mattos, M. K., Hagan, T. L., \& Donovan, H. S. (2017). Comparison of symptoms and quality of life in recurrent ovarian cancer by rural/urban residence: Ancillary analysis of GOG-0259. International Journal of Gynecological Cancer, 27 (Supplement 4), ESGO07-0436, 262.

3. Belcher, S. M., Sereika, S. M., Dodson, Z., Mattos, Meghan K., Hagan, Teresa L., \& Donovan, Heidi S. (2017). Comparison of rural versus urban residence for symptoms and quality of life in women with advanced ovarian cancer: Baseline analysis of GOG-0259, an NRG Oncology/GOG study. Journal of Clinical Oncology, 35 (15_Supplement), e18083. doi: 10.1200/JCO.2017.35.15_suppl.e1803

4. Sherwood, P., Ren, D., Given, C. W., Donovan, H., Weimer, J., Belcher, S., Given, B. (2016). The impact of caregivers' depression on their physical health. Psycho-Oncology, 25 (Special Supplement S3), 24. doi: 10.1002/pon.4272

5. Belcher, S., Low, C., Posluszny, D., Donovan, H. (2016). Correlates of psychological distress, health behaviors, and benefit finding in survivors of multiple primary cancers: Results from the 2010 LIVESTRONG Survey. 8th Biennial Cancer Survivorship Research: Innovation in a Rapidly Changing Landscape Abstract Book, A-61, 41-42.

6. Belcher, S. M., Klem, M. L., Cohen, S. M., Hausmann, E., Donovan, H. S., \& Schlenk, E. A. (2016). Examining the relationship between multiple primary cancers and cancer-related distress: A systematic literature review. Journal of Clinical Oncology, 34 (Supplement 3S), 214. doi:10.1200/jco.2016.34.3_suppl.214

7. Belcher, S., Arida, J., Campbell, G., Hagan, T., Skrovanek, E., \& Donovan, H. S. (2015). Exploring well-being, social support, and financial vulnerability in women with recurrent ovarian cancer who report more than one primary cancer diagnosis within the past 3 years. Nursing Research, 64 (2), E68. doi: 10.1097/NNR.0000000000000089

8. Skrovanek, E., Hagan, T., Campbell, G., Belcher, S., Arida, J., Ackison, G., \& Donovan, H. (2015). Influence of causal attributions on cancer-related distress. Nursing Research, 64 (2), E126-E127. doi: 10.1097/NNR.0000000000000089

\subsection{PROTECTION OF HUMAN SUBJECTS}

\subsubsection{Human subjects involvement and characteristics}

We anticipate that approximately 440 MPC survivors will participate in this research. Inclusion criteria: cancers diagnoses in adulthood ( $\geq 18$ years old); history of two or more primary cancers; first diagnosis consistent with one of the seven most prevalent first cancers experienced by 
male/female MPC survivors: female breast, prostate, colorectal, urinary bladder, uterine, melanoma, kidney and renal pelvis, lung/bronchus, oral cavity and pharynx, thyroid, or ovary; stage I-III cancers (both first and second primary cancers); between 1-10 years following active treatment/no evidence of disease; and able to read and complete questionnaires in English. Exclusion criteria: non-melanoma skin cancer; in situ cancers; stage IV cancers; and history of recurrence.

Vulnerable participants (i.e., fetuses, pregnant women, children, or institutionalized individualized) are not included in this study. Of note, issues relevant to pediatric survivors of multiple primary cancers are likely to be fundamentally different from adult survivors. MPC survivors who experienced cancer as children warrant additional future study.

\subsubsection{Sources of materials}

Data will be collected in the form of self-reported questionnaires, either via pencil and paper or online via Qualtrics, based on individual preference. In addition, disease and treatment data will be extracted from participants' medical records. All data will be identified only by code numbers (participant IDs) and will be stored in secure locations, including locked file cabinets and password-protected computers and databases. Participant IDs will be linked to participants' names in a password-protected file that is accessible only to the PI and research team.

\subsubsection{Recruitment}

We will utilize the Cancer or Cancer-like Blood Disorder Registry (UPCI protocol \#03-038) in order to contact individuals with information about the study. Personnel from the UMPC Network Cancer Registry (see Appendix C for letter of support) will first match registry participants meeting the study's criteria to the UPCI protocol 03-038 patients. For living patients in common between these data sets, our research team will be provided with the names and mailing addresses for the matching patients in order to conduct the recruitment mailings ourselves. If necessary to achieve recruitment goals, our next step would be to utilize the honest broker system within the larger UPMC Network Cancer Registry, facilitating mailings from physician 
champions under the supervision of the study PI to introduce the study to prospective participants who fall within our inclusion criteria.

Based on Dillman's well established and validated approach, MPC survivors meeting the eligibility criteria according to the UPMC Cancer Registry will receive a series of follow-up contacts (see study flow chart in Figure 3). On Day 1, letters will be sent from physician champions on behalf of the study team to introduce prospective participants to the study and invite participation (see sample letter in Appendix B). Each contact will provide potential participants with an easy email or phone opt-out option, if they wish to be removed from the contact list, and any participant who returns questionnaires will not receive further reminders.

For individuals who choose not to participate in the study, we request that they complete a refusal form, that will include 5 general questions to help us compare our study sample to those who declined participation, helping us understand possible sample bias.

If individuals do want to participate in the study, they can respond accordingly by either returning an enclosed postage-paid card, calling the study line, or emailing the PI. They can choose to complete the survey in one to two ways: 1) paper/pencil or 2) web-based via Qualtrics. Ongoing communication will occur via the mode of participation initiated by and contact information provided by the participant.

For paper/pencil selection, participants will be sent a paper copy of the consent (see Appendix B) and survey (see Appendix A). The consent will state that by completing and returning the completed questionnaire, individuals have agreed to participate in the study, and PI contact information will be provided for questions at any time. If a completed survey is not received back, a reminder postcard will be sent followed by replacement questionnaires. A thank you card and the $\$ 5$ Amazon.com gift card code will be mailed to participants upon receipt of completed questionnaires.

For online survey selection, participants will be provided with a link to an electronic copy of the consent, which may be viewed within the survey and also downloaded (see Appendix B). 
Online consent on Qualtrics is a hard stop before participants can move forward with the link to complete the survey online. Qualtrics is a system for web-based data collection and is approved by the University of Pittsburgh IRB. The consent will state that by completing and submitting the online questionnaire, individuals have agreed to participate in the study. The question will be repeated in the actual survey platform. PI contact information will be provided for questions at any time. If no response or partial response (e.g., participant initiated but did not complete the survey), reminder emails will be sent following the initial email. A thank you email and the $\$ 5$ Amazon.com gift card code will be sent to participants upon completion of the survey via provided emails.

Surveys are completed at one time point, so retention strategies are not included. Every effort has been made to make paper and online surveys as similar as possible.

\subsubsection{Potential risks and adequacy of protection against risks}

One potential risk is a breach of confidentiality. To protect participants' privacy, only members of the research team will be aware of individuals' participation in this research study. Participant names will not be included on the paper or electronic questionnaires they complete or the information collected from medical records. All data will be kept in secure, locked file cabinets at the School of Nursing. A username and password will be used to access secured survey data in Qualtrics. All information will be identified only by a study ID number. The information linking ID numbers with identifiable information will be kept separate from the research records; paper data and will be stored under lock and key, and identifiable linkage code data will be stored in a Box cloud folder separate from the deidentified data. The PI will manage access to the identifiable data; access will be provided only to team members who require access for study-related work. All researchers involved in this study have been thoroughly trained and are up to date on online privacy modules. Individual identities will not be revealed in any description or publications of this research, and data will only be presented in aggregate. 
Another possible risk of this research study may include stress from having to complete the questionnaires. Participants are advised that, if the questions induce stress or discomfort, they can take a break from completing the questionnaires and do not have to complete all individual questions. If completing the survey online, they are advised that their data will not be lost if they take a break. It is emphasized that survey responses are not sent to their healthcare providers and that they should contact or see their healthcare team if they have any questions or concerns about physical or emotional symptoms, cancer, or medications or if they experience new symptoms or increase in existing symptom severity. It is estimated that survey completion will take approximately $30-45$ minutes.

\subsubsection{Cost-to-benefit statement}

In light of the relatively minor risks associated with the study (e.g., minor distress or physical discomfort, breach of confidentiality), the risk-benefit balance is reasonable.

Participants will likely no acquire direct benefit from study participation. The primary benefit of this study lies in the importance of knowledge to be gained.

1.8.5.1 Importance of knowledge to be gained. The number of cancer survivors, specifically the number of individuals with MPC, is growing dramatically in the United States (American Cancer Society, 2014; Bluethmann et al., 2016; DeSantis et al., 2014; Fraumeni et al., 2006; National Cancer Institute, n.d.-c, n.d.-b), increasing the need to identify the characteristics of those diagnosed with MPC and the potentially modifiable self-management behaviors that interrupt negative stress response pathways and have potential to optimize health and wellbeing. Data from the proposed study will allow investigators to target particular behavioral mechanisms related to prolonged stress in MPC survivors most vulnerable to prolonged stress and the cascade of negative outcomes. The goal is that study data will provide findings that can be used to improve care for future patients and survivors and to inform future intervention studies for this population. 


\subsection{SUMMARY OF STUDY}

\subsection{CHANGES TO PROPOSED STUDY}

This section is intended as a bridge between the proposed study, as approved by the committee, and the actual study as it was conducted. Reviewed here are the major changes to the originally proposed study related to recruitment and resulting data collection procedures.

\subsubsection{Recruitment}

The original plan for our study team to directly recruit the majority of our participants through the voluntary Cancer or Cancer-like Blood Disorder Registry (UPCI protocol \#03-038) was not possible, as we were notified pre-study launch that the protocol had been permanently closed. Therefore, we used our previously identified alternative approach to recruit participants by partnering with the UPMC Network Cancer Registry's honest broker system (IRB \#HB015) to contact prospective participants from the larger cancer registry sampling frame. The UPMC Cancer Registry draws from accredited hospitals who actively follow patients, including: Altoona, East, Horizon, McKeesport, Magee, Northwest, Passavant, Presbyterian Shadyside, and St. Margaret.

2.1.1.1 Additional participation criteria. In addition to our originally planned inclusion and exclusion criteria, additional exclusion criteria were required by the UPMC Registry protocol. Based on requests from physician leaders identified prior to study launch, the UPMC Registry staff compared the list of potentially eligible participants $(n=2233)$ to the corporate "opt out" database maintained by the Medical and Health Sciences Foundation Office and removed 62 $(2.8 \%)$ of the potentially eligible patients who were common between lists. Per UPMC Registry protocol, each physician champion enlisted as a clinical expert for their respective disease site(s) (see 1.4.3) was also provided with a list of potentially eligible patients from his or her disease specialty. Opportunities were provided for each physician champion to review the list and deem patients not appropriate for contact about this study; $46(2.1 \%)$ potentially eligible 
participants were removed based on this review. Lastly, 10 prospective participants who were mailed study letters of invitation were unable to be contacted regarding study participation, as they were either deceased or noted by their families as unable to participate due to dementia; these 10 individuals were subtracted from the number of potentially eligible participants (see Manuscript 3, section 3.3.4.1 for additional details).

2.1.1.2 Dillman's Tailored Design Method. The launch of our initial mailings was delayed due to the previously described changes in the source of recruitment and overlap with major holidays. Consequentially, the timing of reminder mailings to nonrespondents was also delayed until immediately following the start of the new year. See Figure 7 in Manuscript 3 for the revised study flow.

Because we were contacting potential participants using UPMC Registry's honest broker system, we were not provided with an individual's contact information until he or she first contacted us about the study. Thus, our originally planned series of four follow-up contacts with potential participants who did not respond to initial mailings was not possible. Balancing feasibility of UPMC Registry workload and privacy of potential participants being approached through the UPMC Registry, we partnered with the UPMC Registry to send one additional reminder postcard to prospective participants who had not responded to the initial study mailings (IRB approved).

For individuals who requested to complete surveys on paper but had not returned their completed survey by Day 21 , our final contact was accomplished via the mode of communication that was used or preferred by each participant. For example, if an individual had called the study telephone number to request a paper survey, our final contact consisted of a reminder telephone call to answer any questions and encourage participation.

2.1.1.3 Nonresponse and return to sender. We provided UPMC Registry personnel with updated lists of individuals who had contacted us about study participation. The UPMC Registry's honest broker system tracked individuals who did not respond to study mailings. They 
also identified updated addresses and resent initial letters of invitation for $n=114$ individuals whose first letter had been returned as undeliverable. UPMC Registry personnel tracked undeliverable letters of invitation and reminder postcards.

2.1.1.4 Measures and scoring. Reviews of the planned set of measures were conducted prior to study launch. Based on a balance of participant burden and desired measurement, the following changes were made pre-survey launch.

2.1.1.4.1 Psychological responses. We utilized the Mental Health subscale of the PROMIS Global Health measure (Hays, Bjorner, Revicki, Spritzer, \& Cella, 2009; HealthMeasures, 2017), a scale originally planned for exploratory descriptive purposes, as a measure of the psychological response latent variable. The version of the scale we administered, v1.0, was recently retired. Thus, we followed developer instructions to convert the Global Health data observed in our study to v1.2 format for scoring.

2.1.4.2 Physical health outcomes. The 10-item version of the PROMIS Physical Function was administered, rather than the 20 -item version, to reduce participant burden.

2.1.1.4.3 Personality. The IPIP mini was administered (Donnellan, Oswald, Baird, \& Lucas, 2006), rather than the originally planned 20 item version, to decrease participant burden.

2.1.1.4.4 Exploratory measures. To reduce participant burden and to reduce redundancy among measures, the Index of Self-Regulation and Self-Efficacy for Managing Chronic Disease exploratory measures were not included in our battery of administered instruments.

2.1.1.5 Data sources. In addition to self-reported data and data extracted from the medical record, we incorporated UPMC Cancer Registry clinical and sociodemographic data in our analyses. To maintain consistency with study inclusion criteria based on UPMC Registry queries, first cancer diagnoses are based upon diagnoses recorded in the UPMC Cancer Registry.

Due to feasibility and cost, we were not able to collect and bank biological samples for future analyses. 


\subsubsection{Data analyses}

2.1.2.1 Missing data. Scales were scored per developer instructions. When no information was available regarding number of items required to scale a score, scores were imputed if at least $80 \%$ of scale items were completed.

Four participants who consented to online study participation did not complete the full survey and were dropped from analyses.

2.1.2.2 Model fit indices. In additional to comparative fit indices (CFI), Tucker-Lewis Index (TLI), and root mean square error of approximation (RMSEA), we also used standardized root mean square residual (SRMR) to assess model fit. During analyses for determination of good model fit, we adopted more stringent cut points than were originally proposed for practical fit

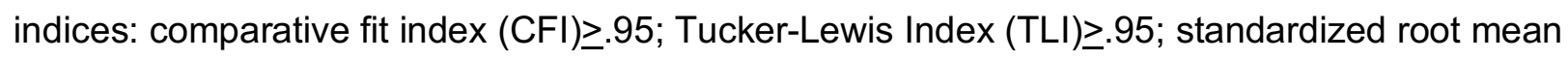
residual $(\mathrm{SRMR}) \leq .08$; root mean square error of approximation (RMSEA) $\leq .08$ (Hu \& Bentler, 1999; Kenny, 2015; Little, 2013).

2.1.2.3 Sample size. We had originally set a sample size goal of $n=440$ to balance cost, feasibility, and analytic power. Ultimately, 215 participants were recruited for this study, and 4 were dropped due to incomplete data (response rate $=15.2 \%$ ). After 5 additional participants were dropped from analyses due to having multivariate outlier data, a final sample size of 206 participants was included in multivariate analyses.

Despite not achieving our originally desired sample size, the SEM for our primary aim remained adequately powered. With the retained sample size of 206 and 309 degrees of freedom, >.99 power remained for either the test of a close fit (i.e., RMSEA $A_{0}=05$ vs. RMSEA $_{A}=.08$ ) or exact fit (i.e., $R M S E A_{0}=0.0$ vs. RMSEA $_{A}=0.05$ ) at a significance level of .05 . 2.1.2.4 Analytic variables for multivariate analyses. Due to sparse cell sizes for race and a large amount of missing data for income, we were unable to retain these planned variables as covariates in analyses. We did, however, include "difficulty making ends meet" as a variable in our multivariate analyses. Clinical variables ultimately examined in Aim 2 included: first cancer 
site and years since first cancer diagnosis (obtained from Cancer Registry data); second cancer site, years since second cancer diagnosis, years since most recent treatment, time between first and second cancer diagnoses, presence of three or more cancer diagnoses (yes versus no), and most recently documented BMI (obtained from medical record review).

2.1.2.5 Aim 3 analyses. To explore individual hei-Q item responses by domain for selfmanagement, responses were collapsed into two categories (disagree and agree) and explored by domain. Mean domain scores (without score alterations) and individual item responses are reported (see Table 14).

To evaluate potentially modifiable health behaviors, we analyzed heiQ items, alcohol use, tobacco use, and BMI measures in detail (see Tables 14-16).

\subsection{CONCLUSIONS, IMPLICATIONS FOR NURSING, FUTURE STUDIES}

This dissertation is composed of three complementary studies that demonstrate a clear and focused progression from an evaluation of the state of the science (Manuscript \#1), to a secondary analysis to explore associations among select model variables in a national sample of MPC survivors (Manuscript \#2), the dissertation study in which we carried out a systematic model evaluation to elucidate key pathways impacting health in MPC survivors (Manuscript \#3), and concludes with a characterization of positive and negative self-management behaviors in MPC survivors (Aim 3; section 4.0). Here we briefly summarize the implications of the preliminary studies prior to focusing on the final dissertation study.

In Manuscript 1 (Belcher et al., 2016), we reviewed and synthesized the literature to determine the relationship between experience of MPC diagnoses and psychological distress in adult cancer survivors. This study identified a lack of research focused on and/or including adults with MPC diagnoses. Across the five studies that met criteria for inclusion in the review, calculated effect sizes supported small but potentially significant increases in psychological distress in survivors of MPC compared to single cancer survivors. Findings from this manuscript were combined with literature in other populations to select and refine variables and pathways 
for the conceptual model. Additionally, noted gaps including lack of racial and ethnic diversity in MPC samples, self-reported clinical data, inconsistent use of valid and reliable measures, inclusion of non-melanoma skin cancer cases, and lack of focus on characterization of MPC survivors at risk for increased distress were addressed in the design of the subsequent dissertation study.

In Manuscript 2 (Belcher et al., 2017), we conducted a secondary analysis of a national cancer survivorship dataset to compare survivors of single cancer versus MPC diagnoses on psychological distress, self-management behaviors, and benefit finding. Having MPC was associated with psychological distress and positive healthcare utilization (i.e., attending medical appointments, monitoring for second cancer, and being up to date on screenings) but not with healthy lifestyle (i.e., regular physical activity, healthier diet, and attempts to take care of health) or benefit finding. The study reaffirmed findings of psychological distress for MPC survivors in a large, mixed-cancer survivor population and allowed for clearer conceptualization of model concepts, including the introduction of self-management as an important consideration in MPC, where survivors often view cancer as a chronic disease (Belcher et al., 2017).

In Manuscript 3, dissertation aims 1 and 2 (see section 3.3), we used structural equation modeling to evaluate hypothesized relationships linking stress to poor health in an adapted psychobehavioral stress response model. The data fit a modified four-factor measurement model, with latent variables including self-management, distress (combined perceived stress and psychological distress), financial toxicity, and functional health (combined social health and physical health). In the expanded model including possible predictors, overweight BMI, graduate education, less neuroticism, and increased social support predicted better self-management; poorer self-management, greater neuroticism, and lower social support predicted increased distress. The findings provide opportunities for intervention work targeting modifiable pathways and for early identification of individuals at increased risk for negative health outcomes. 
To address dissertation aim 3 (see Chapter 4.0), we explored individual positive selfmanagement behaviors and indicators of negative health behaviors (i.e., alcohol use, smoking, and BMI). Self-management item scores were generally high but variable, and rates of obesity were well above population and cancer norms. This exploration of data highlights a need for additional study focused on risk factors associated with positive and negative health behaviors in MPC survivors.

Self-management was the upstream latent variable identified in the final modified model, predicting the other latent variables either directly (i.e., predicting distress) or indirectly (i.e., through distress). In contrast to the common data element instruments recommended by the National Institute of Nursing Research (NINR) (Moore et al., 2016), which are limited to measures associated with health behaviors (i.e., patient activation, self-regulation, self-efficacy for managing chronic conditions, and global health), the internationally-tested heiQ, selected to measure self-management in this study, was designed specifically to measure outcomes following health education and self-management programs (Elsworth et al., 2015; Osborne et al., 2011, 2007) and directly assess the range of self-management domains recommended to maintain wellness.

In this study, the measures used to capture the hypothesized latent variables of perceived stress and psychological response loaded onto the same factor, which we called distress. Future studies should evaluate the validity of combining these measures. It is possible that the high correlations among these sets of variables was a consequence of study design and/or measurement issues. The original conceptualization of the perceived stress latent variable, the perception that one's demands overwhelm one's coping resources (Lazarus \& Folkman, 1984), was meant to capture current stressors of general life demands, cancerspecific stress, and cancer worry. Conversely, the psychological response latent variable was mean to capture the longer-term (maladaptive) responses to the chronic stress of cancer (National Comprehensive Cancer Network, 2018a) and included measures of depressive 
symptoms, anxiety, and mental health. It is possible that the cross-sectional nature of this study precluded distinguishing between these short- and long-term responses in MPC survivors who had been living with their diagnoses for, in some cases, multiple years. Similarly, social role ability and physical health measures loaded onto a combined factor, which we conceptualized as functional health. Ability to participate in social roles and activities does reflect a functional ability, but, again, further research is necessary to evaluate the validity of combining these different measures into a single latent variable. Finally, the PROMIS Global Mental Health measure we attempted to incorporate into this model loaded across many of the latent variables in the model, preventing us from using it in multivariate analyses.

Importantly, these collapsed latent variables demonstrated clear associations among each other in the directions that we had hypothesized. Distress, which was predicted by selfmanagement behaviors, significantly predicted both increased financial toxicity and poorer health outcomes. We hypothesize that increased distress may be impairing an individual's productivity, leading to financial toxicity, and that financial toxicity may be impacting an individual's physical health (e.g., through medication adherence). Further, we hypothesize that distress may be affecting functional health through biological pathways (e.g., triggered glucocorticoid receptor resistance, immune dysregulation, and risk for disease) (Cohen et al., 2012). It is also possible that mechanisms impacting an individual's risk for MPC (e.g., genetic predisposition, previous cancer treatment, negative health behaviors, etc.) may also influence health outcomes in this survivor population. Future studies should identify the individual and interacting influence of these different mechanisms.

Additionally, BMI, education, neuroticism, and social support were significant covariates for this variable. Notably, the rate of obesity in this sample $(40.7 \%)$ is higher than both that of the general U.S. population (36.5\%) and cancer survivors (31.1\%) (Centers for Disease Control and Prevention, 2017; National Cancer Institute, 2018b). Also, we recruited a sample of 
participants with less education than is typical of previous MPC study samples $(38.8 \%$ with high school level or less), making this a sample that is more generalizable to the U.S. population.

Not all of the originally hypothesized pathways (see Figures 10 and 11, Section 3.3) were found to be statistically significant. The direct pathway between self-management behaviors and financial toxicity was neither significant nor was it in the direction we had hypothesized; for financial toxicity, distress was a more important direct predictor, while distress mediated the relationship between self-management and financial toxicity. Also, while the coefficient did reflect the hypothesized direction, self-management behaviors also were not a significant direct predictor of functional health. The relationship, again, was mediated by distress.

Additional strengths and limitations are acknowledged in this study. Fortunately, we had adequate power to conduct the planned structural equation modeling despite a lower than expected response rate to study mailings (15.2\%). Study participants were similar to participants in previously recruited national MPC samples (e.g., similar age, gender, ethnicity, marital status), despite being recruited from a regional cancer registry. Additionally, study participants did not differ from nonparticipants identified by the Cancer Registry as potentially eligible for participation in this study on key variables including age, gender, race, marital status, primary payer at first diagnosis, years since first cancer diagnosis, and first cancer site (see Table 7 in Section 3.3).

Some differences did exist between our sample and other published reports. First, as a result of our inclusion criteria, this sample of patients was, on average, closer to their first cancer diagnosis $(M=5.8$ years, $S D=2.9)$ than other published samples $(11-17$ years $)$, providing the opportunity to examine a new cohort of MPC survivors not previously included in this body of literature (Belcher, Low, Posluszny, Kramer, \& Donovan, 2016; Burris \& Andrykowski, 2011; Gotay et al., 2007; Thong et al., 2013). This study also included cancer sites not frequently represented in MPC literature (i.e., lung and thyroid cancer). Additionally, this sample had 
higher average BMI and rates of obesity $(41 \%)$ when compared to other MPC samples (Burris \& Andrykowski, 2011; Thong et al., 2013). It is not clear whether this reflects the general increase in BMI across the U.S. or whether it is an emerging and unique problem among MPC survivors. While not successful in recruiting a racially and ethnically diverse sample, we were successful in recruiting a sample of participants with a broad range of educational attainment, which is representative of the broader U.S. population (Ryan \& Bauman, 2016). Importantly, educational attainment was identified as an important predictor of self-management in Aim 2 covariate analyses.

The data in this study support healthy self-management behaviors as vitally important to positive health outcomes in MPC survivors. Clinicians caring for MPC survivors should assess persons with high-risk BMI, low educational levels, low social support, and greater neuroticism for poor health behaviors and distress, both of which are modifiable. Assessments should include diet, activity, caloric balance, and brief distress screenings. The National Comprehensive Cancer Network's survivorship and distress clinical practice guidelines (National Comprehensive Cancer Network, 2018b, 2018a) provide direction on ways to assess and intervene in these areas. Clinicians should also increase their awareness of financial toxicity and should engage their patients in care value discussions. As evidenced by the expertise needed to address these key clinical priorities in MPC patients, collaboration among specialists (e.g., nutritionists, behavioral change experts, mental health professionals, financial experts, primary care providers, and oncologists) is key.

As recognized by recent literature documenting increasing rates of MPC in cancer survivors (Davidson, 2017; Murphy, Gerber, \& Pruitt, 2017), clinical trial eligibility criteria should be evaluated to ensure representativeness of the U.S. cancer survivor population. Whenever possible, MPC status should not be an explicit exclusion criterion for clinical trial participation. Also, organizations should strive to create systems and policies that allow for continuity of care among providers for these medically complex patients. 
Future studies should evaluate this refined model for studying health outcomes in adults with MPC, with a particular focus on self-management and financial toxicity, two newly highlighted areas of importance. Biological pathways also are an untapped line of inquiry in this patient population and could lead to increased understanding of the mechanisms linking distress to poor outcomes in this model. It is also important to understand how risks for poor health outcomes may differ among different subsets of MPC survivors (e.g., childhood versus adulthood diagnoses; MPCs associated with genetic cancer syndromes versus treatmentrelated MPCs versus MPCs associated with risky behaviors). Future research should move to longitudinal studies to 1) establish temporal relationships among key variables, 2) understand how MPC survivorship risk factors change over time, and 3) identify vulnerable phases in the MPC survivorship trajectory.

Future MPC studies should also focus on novel recruitment methods for this hard to reach patient population, as survivors do not all attend specialty MPC clinics where focused recruitment could occur. Future studies must also focus on conducting studies that are sensitive to the challenges and perspectives of more diverse groups of MPC survivors. With increased demands being placed on people's time and attention, survey response rates have been declining and costs have been rising (National Science Foundation, n.d.), and this type of data collection may be becoming outdated. Attention should also be paid to addressing the reasons for nonparticipation identified in this study (i.e., perception by elderly MPC survivors that their input is not valuable; lack of knowledge/awareness of their MPC cancer status; and concerns about privacy and allowing access to medical records). 


\subsection{MANUSCRIPTS}

This dissertation is comprised of two published manuscripts and one pending submission manuscript draft.

Manuscript 1 (see Appendix E):

Belcher, S. M., Hausmann, E. A., Cohen, S. M., Donovan, H. S., \& Schlenk, E. A. (2016). Examining the relationship between multiple primary cancers and psychological distress: A review of current literature. Psycho-Oncology, 26(7), 2030-2039. https://doi.org/10.1002/pon.4299

Manuscript 2 (see Section 3.2 for the manuscript version accepted for publication and Appendix $F$ for link to final publication):

Belcher, S. M., Low, C. A., Posluszny, D. M., Schear, R., Kramer, R. E., \& Donovan, H. S. (2017). Psychological distress, health behaviors, and benefit finding in survivors of multiple primary cancers: Results From the 2010 Livestrong survey. Oncology Nursing Forum, 44(6), 703-711. https://doi.org/10.1188/17.ONF.703711

Manuscript 3 (see Section 3.3):

Belcher, S. M., Donovan, H. S., Bovbjerg, D. H., Sherwood, P. R., Campbell, G. B., \& Sereika, S. M. (n.d.). Adapting a psychobehavioral stress-response model to characterize risks in survivors of multiple primary cancers. In preparation. 


\subsection{DISSERTATION MANUSCRIPT 1: REVIEW OF LITERATURE}

The full text of this manuscript was reproduced with permission from Psycho-Oncology and can be found in Appendix E. 


\subsection{DISSERTATION MANUSCRIPT 2: PILOT TESTING ASSOCIATIONS AMONG KEY VARIABLES}

Presented here is the full text version of the manuscript accepted for publication, which was subsequently published in Oncology Nursing Forum. The final publication may be accessed at: http://store.ons.org/article/find?doi=10.1188/17.ONF.703-711

Psychological Distress, Health Behaviors, and Benefit Finding in Survivors of Multiple Primary Cancers: Results from the 2010 LIVESTRONG Survey

\subsubsection{Abstract}

Purpose: To evaluate whether survivorship of multiple primary cancers (MPC) is associated with psychological distress, health behaviors, and benefit finding.

Design: Secondary analysis of the 2010 LIVESTRONG cross-sectional survey.

Setting: Online survey.

Sample: 238 MPC and 3,295 single cancer survivors.

Methods: Chi-square and t-tests for group comparisons. Multivariate linear regression, adjusted for covariates, for associations between variables.

Main Research Variables: MPC versus single cancer; psychological distress, health behavior (healthy lifestyle and positive healthcare utilization), and benefit finding scores.

Findings: Survivors of MPC, compared to single cancer survivors, were significantly older, less likely to have a spouse/partner, further out from original cancer diagnosis, less likely to be employed full-time, and differed by cancer diagnoses and survivorship stage. MPC was associated with significantly higher psychological distress and healthcare utilization but not healthy lifestyle or benefit finding.

Conclusions: Relative to those with single cancers, MPC survivors are at increased risk for psychological distress and are more likely to receive recommended cancer screenings.

Additional research is needed to understand mechanisms surrounding psychological distress in MPC survivors. 
Implications for Nursing: Targeted distress screening in MPC survivors may allow for early identification and interventions to ameliorate distress and reduce negative downstream health effects.

Knowledge Translation: Nurses should assess for previous cancer histories and recognize that survivorship experiences may differ between MPC and single cancer survivors. MPC survivors have increased psychological distress risk and may have needs related to living with cancer as a chronic illness. Further study of psychological distress mechanisms in MPC survivors is warranted.

Keywords: multiple primary cancers, cancer survivorship, psychological distress, health behaviors, benefit finding 


\subsubsection{Background}

With improved cancer screenings and treatments, the United States cancer survivor population is predicted to reach 20.3 million people by 2026 and 26.1 million by 2040 (American Cancer Society, 2016; Bluethmann et al., 2016; Jemal et al., 2017). As survival increases following cancer diagnoses and general effects of aging occur, cancer survivors, however, are facing additional serious health issues including subsequent malignant neoplasms. One in five cancers diagnosed in the United States will occur in someone who has a previous cancer diagnosis, and these multiple primary cancers (MPCs) are a major cause of morbidity and mortality in cancer survivors (De Gonzalez et al., 2011; Morton et al., 2014).

A second, or multiple primary, cancer (MPC) is the occurrence of a new cancer that is histologically distinct from the original primary cancer and has been ruled out as metastatic disease of the primary tumor (Begg, 1999). An example of someone who is an MPC survivor is an individual who experiences breast cancer and later presents with a new diagnosis of ovarian cancer. Contrast this with a woman with breast cancer that metastasizes to the bone, which is diagnosed as metastatic spread of the original breast cancer; this would not be considered an MPC. Risk of developing subsequent MPCs varies by site of first primary cancer, age at first cancer diagnosis, environmental and behavioral exposures, genetic susceptibility, and cancer treatment effects (American Cancer Society, 2009, 2012; Morton et al., 2014).

The National Academy of Medicine, professional organizations, cancer survivorship advocates, clinicians, and scientists have called for an increased focus on addressing the health and psychosocial needs of the growing population of cancer survivors (American Cancer Society, 2016; Klein et al., 2014; Knobf et al., 2015; K. D. Miller et al., 2016; Mullan, 1985, 2016; National Academy of Sciences, 2006), and the MPC population represents an understudied and at risk group in critical need of additional research. While having a single cancer has been linked to risks for psychological distress (Holland et al., 2013; Mitchell et al., 2011; National Comprehensive Cancer Network, 2015), poor health behaviors (Mowls, Brame, Martinez, \& 
Beebe, 2016; Underwood et al., 2012), and poor physical health outcomes (Ness, Wall, Oakes, Robison, \& Gurney, 2006; Stein, Syrjala, \& Andrykowski, 2008) that can persist throughout cancer survivorship, an initial small body of literature is evolving to suggest that the risk for these poor outcomes appears to be even greater in MPC survivors (Andrykowski, 2012; Belcher, Hausmann, Cohen, Donovan, \& Schlenk, 2016; Burris \& Andrykowski, 2011; Dowling et al., 2013; Gotay, Ransom, \& Pagano, 2007; Thong et al., 2013). Most cancer survivorship literature, however, has been conducted irrespective to number of cancer diagnoses, limiting our ability to understand potentially unique experiences and needs in this survivor subset. Additionally, no studies of MPC cancer survivors to date have analyzed a large national dataset, such as the LIVESTRONG survey, that focuses entirely on post-treatment cancer survivorship issues.

Many cancer survivors experience persistent late and/or long-term effects of cancer and cancer treatment (National Comprehensive Cancer Network, 2017). Uncontrolled psychological distress in cancer survivors is known to negatively impact quality of life, adherence to surveillance recommendations, and engagement in health promotion activities (National Comprehensive Cancer Network, 2016). Previous cancer survivorship literature has demonstrated that healthy lifestyle behaviors are associated with decreased chronic illness and improved health and quality of life (Blanchard, Courneya, \& Stein, 2008; Davies, Batehup, \& Thomas, 2011; Ford et al., 2009). Benefit finding, the perception of positive changes such as renewed appreciation for life following adversity, has been found in single cancer populations and may also be related to positive health behavior change and psychological adjustment (Harper et al., 2007; Hawkins et al., 2010; Kanera et al., 2016; Low et al., 2014). Previous cancer survivorship literature has been conducted without consideration of patients' history of multiple primary cancers, but early literature suggests that this growing population of MCP survivors may be at an increased health risk, highlighting a critical need to build the science to 
identify potentially modifiable risk and protective factors contributing to health outcomes in this unique cancer survivor population.

The purpose of this secondary analysis of 2010 LIVESTRONG national cancer survivorship survey data is to evaluate whether MPC survivorship is associated with psychological distress, health behaviors, and benefit finding. We report 1) sociodemographic and clinical differences between survivors of single cancers versus MPCs and 2) the contribution of MPC survivorship to psychological distress, health behaviors, and benefit finding after controlling for important covariates. Findings from this study are used to make recommendations to support MPC survivors that are applicable to a wide range of nurses.

\subsubsection{Materials and Methods}

3.2.3.1 Data. The 2010 LIVESTRONG Survey for People Affected by Cancer was a crosssectional survey fielded online by LIVESTRONG between June 2010 and March 2011.

Constituents of LIVESTRONG were notified about the survey via email, Twitter, and Facebook, and partner organizations, state cancer coalitions, and comprehensive cancer centers shared survey information with their respective constituents and/or patients (Beckjord et al., 2014; Campbell et al., 2011; Shapiro et al., 2009) .

The 2010 LIVESTRONG survey was developed in response to the Institute of Medicine Report recommendations (National Academy of Sciences, 2006) that nonprofit organizations increase their support of cancer survivorship research and associated mechanisms and was aimed at examining post-treatment survivorship issues. The LIVESTRONG Foundation developed items for the preceding 2006 LIVESTRONG survey through a multi-year formative research process, during which experts and cancer survivors were consulted to incorporate challenges faced by cancer survivors. Many of the 2006 LIVESTRONG survey items were retained in the 2010 survey following a RAND Corporation analysis that examined survey response patterns and content (Rechis et al., 2011). Main topic areas in the 2010 survey included physical, emotional, and day to day concerns as well as meaning making, information 
seeking, and advocacy and engagement. Additional details regarding survey development, participant recruitment, and survey administration have been previously published (Beckjord et al., 2014; Low et al., 2014; Posluszny et al., 2015; Rechis et al., 2011).

3.2.3.2 Sample. The parent study received Institutional Review Board (IRB) approval (Rechis et al., 2011), and this analysis of de-identified 2010 LIVESTRONG survey data was approved by the University of Pittsburgh IRB. The initial survey included 4,192 post-treatment adult cancer survivors whose data were considered for this study. Sample selection by single and multiple cancer groups is described below and is presented as a flow chart in Figure 5.

Multiple versus single cancer diagnosis. Survey respondents were asked to report "type of cancer (primary site)" and could choose from an extensive 88-item checklist of cancer types. Respondents were also asked to separately identify any "additional cancer diagnoses or recurrences." As presented in Figure 5, a priori decisions were made to exclude nonmelanoma skin cancer cases in both single and MPC groups. Additional exclusion criteria for the MPC group included: second cancer identical to first primary cancer (i.e., recurrence); definite or probable metastatic disease for common sites of cancer metastases (i.e., bone, liver, lung, and brain) (National Cancer Institute, 2013); and/or unclear, missing, “I don't know," or non-cancer "other" diagnoses that were not actual cancer diagnoses (e.g., "stroke"). 


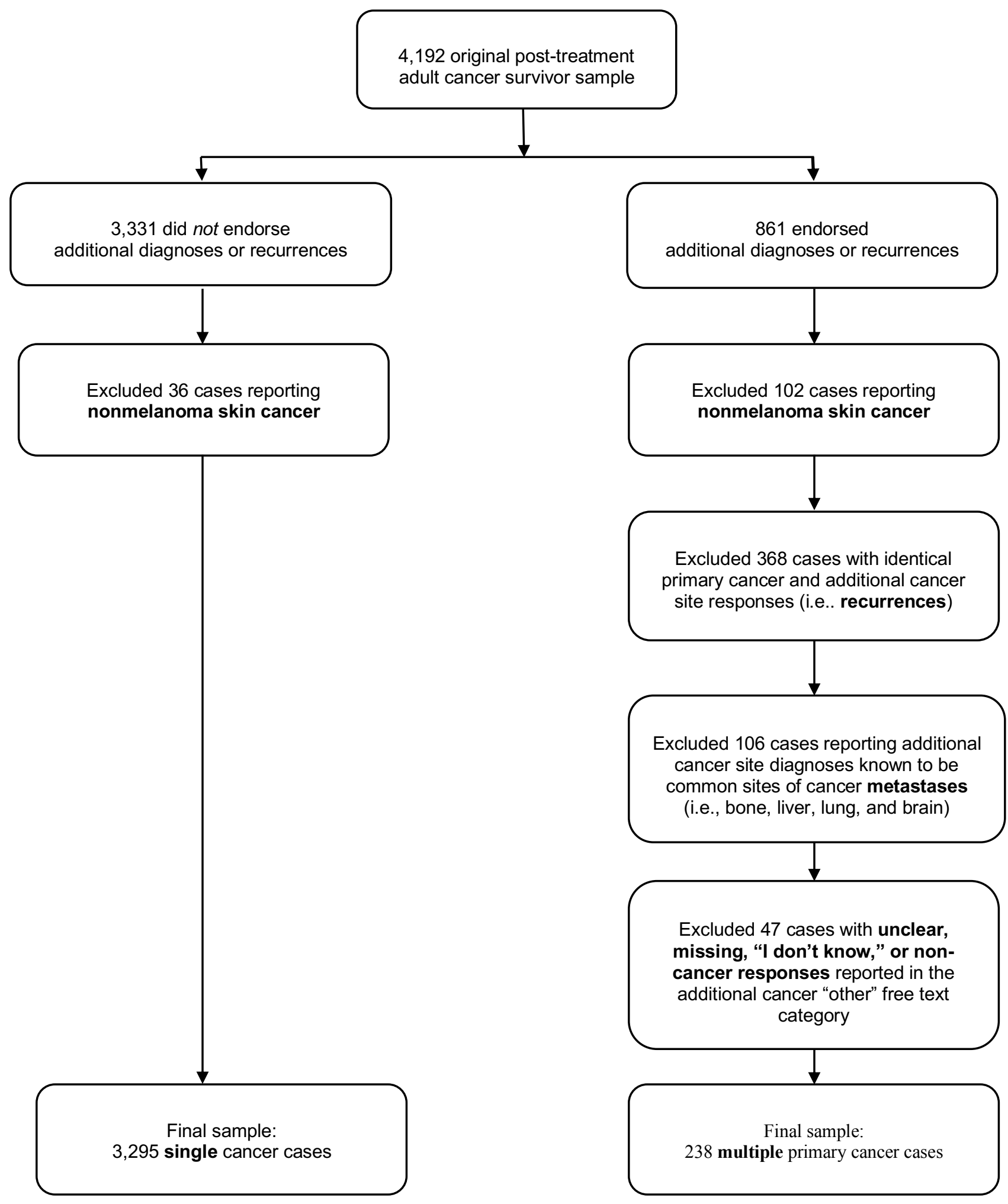

Figure 5. Flow chart of process resulting in $(n=3553)$ post-treatment adult cancer survivor sample 


\subsubsection{Variables of Interest.}

Cancer diagnoses. Classification of respondents as either single or MPC survivors is previously described under sample.

The following categories of variables were assessed in the LIVESTRONG survey by asking: "Since completing treatment, have any of the following statements been true for you as a result of your experience with cancer?" A series of statements followed, to which respondents could answer "yes," "no," or "I don't know." "I don’t know" replies were treated as missing data in this study. Individual survey item endorsements were used to compute sum scores for the four survey outcome categories of interest.

Psychological distress, health behaviors, and benefit finding. Psychological distress included 8 items pertaining to anxiety; worry, tension, or stress; preoccupation with cancer; worry about dying from cancer; worry about cancer recurrence; depression; and mood swings. Health behaviors were divided into two categories: 1) healthy lifestyle and 2) positive healthcare utilization. Four healthy lifestyle behavior items included leading a healthier lifestyle; regular physical activity (2-3 times per week); healthier diet; and attempts to take care of health. Positive healthcare utilization included 3 items including attending regular medical appointments; monitoring for second cancer; and being up to date on recommended cancer screenings. Six benefit finding items included greater appreciation for life; recognition of what's important in life; renewed spirituality; ability to better deal with stress; better coping; and overall feeling like a better person.

Sociodemographic and clinical variables. Sociodemographic variables included age at survey; gender; race; partner status (i.e., single, divorced/widowed, and married); children under 18 living in the home; educational status; total household income; and employment status. Cancer-related clinical variables included age at initial cancer diagnosis; years since diagnosis; first primary cancer diagnosis (included categories for top 5 most prevalent diagnoses represented by respondents [breast, testicular, colorectal, hematologic, prostate]; remaining 
diagnoses were represented by "other"); years since last treatment; stage of survivorship (i.e., currently on treatment, living with cancer as a chronic illness, $<1$ year post treatment, 1-5 years post treatment, $>5$ years post treatment, prefer not to answer/unsure); and cancer treatment received (i.e., no chemo, chemo only, chemo plus surgery and/or radiation). The selection of these predictor variables was driven by critical variables identified in the MPC literature (Andrykowski, 2012; Belcher et al., 2015; Belcher et al., 2016; Burris \& Andrykowski, 2011; Dowling et al., 2013; Thong et al., 2013).

3.2.3.4 Analyses. Descriptive statistics were used to characterize the sample and key variables of interest. To compare characteristics between SC and MPC groups, we used independent sample $t$-tests for continuous variables and $\chi^{2}$ for categorical variables. Post-hoc contingency table analyses using Pearson $\mathrm{x}^{2}$ testing were conducted for categorical variables reaching statistical significance, and Bonferroni adjusted p-values were calculated to correct for Type I error.

Predictor variables of interest were selected a priori based on the literature and were included in the empirically driven multivariate analyses. Multivariate linear regression analysis with listwise deletion was used to develop models for predicting the overall categories of psychological distress, healthy lifestyle behaviors, positive healthcare utilization, and benefit finding, adjusted for both statistically $(p<.05)$ and theoretically significant covariates. Variables were included as model covariates if they 1 ) were related to MPC in bivariate analyses at $p<.05$, or 2) were associated with outcomes in previously published work (i.e., were statistically or theoretically significant). Standardized $\beta$ and $p$-values are reported for multivariate linear regression models.

The data were analyzed using IBM SPSS Statistics software, version 22. All tests were 2-tailed, and statistical significance criterion threshold was set at $p<.05$ unless otherwise noted for Bonferroni corrections. 


\subsubsection{Results}

Descriptive statistics are displayed in Table 3 for both single $(n=3,295)$ and MPC $(n=238)$ groups. MPC participants differed significantly from those with single cancer diagnoses in that they were older at the time of survey completion and were further out from their initial diagnosis. Additionally, groups differed statistically by partner status, employment status, type of first primary cancer diagnosis, and stage of survivorship. Specifically, those with MPC were less likely to have had breast cancer and were more likely to have had one of the less common cancers represented in the dataset, "other" as a first primary cancer diagnosis. First primary cancer diagnoses most frequently represented in the other category for MPC survivors included ovarian, uterine, and thyroid cancer. Breast cancer and melanoma were the two most commonly reported second primary cancer diagnoses for MPC survivors. MPC survivors were also more likely than single cancer survivors to endorse living with cancer as a chronic illness when identifying their stage of survivorship. Being divorced or widowed was more common in MPC survivors, but this difference was not significant after Bonferroni adjustment.

Table 3.

Sample Sociodemographic and Clinical Characteristics by Single Cancer and Multiple Primary Cancer Groups $(N=3,533)$

\begin{tabular}{|c|c|c|c|c|c|}
\hline \multirow[b]{2}{*}{ Variables } & $\begin{array}{c}\text { Single } \\
\text { Cancer } \\
\mathrm{n}=3295\end{array}$ & $\begin{array}{c}\text { Multiple } \\
\text { Primary Cancers } \\
n=238\end{array}$ & \multirow[b]{2}{*}{ Statistic (df) ${ }^{\mathrm{a}}$} & \multirow{2}{*}{$\begin{array}{c}95 \% \text { CI } \\
\text { or p-value }\end{array}$} & \multirow{2}{*}{$\begin{array}{c}\text { p-value } \\
\text { for } \\
\text { Post-hoc } \\
\text { testing }\end{array}$} \\
\hline & \multicolumn{2}{|c|}{ Mean (SD) } & & & \\
\hline Age at Survey, years & $48.4(12.5)$ & $53.3(11.3)$ & $\mathrm{t}(3519)=-5.89$ & $-6.54,-3.28^{*}$ & \\
\hline Age at Initial Diagnosis, years & $42.9(13.8)$ & $41.8(15.2)$ & $t(3488)=1.15$ & $-0.76,2.91$ & \\
\hline \multirow[t]{2}{*}{ Time Since First Diagnosis, years } & $5.1(6.5)$ & $11.4(10.3)$ & $t(3372)=-13.53$ & $-7.21,-5.39^{*}$ & \\
\hline & \multicolumn{2}{|c|}{ Number $(\%)$} & & & \\
\hline Gender & & & & & \\
\hline Female $(n=2218)$ & $2060(62.9)$ & $158(66.7)$ & $\chi(1)=1.37$ & .24 & \\
\hline \multicolumn{6}{|l|}{ Race } \\
\hline White $(n=3074)$ & $2865(92.4)$ & $209(91.7)$ & $\chi(1)=.16$ & .69 & \\
\hline Partner Status ${ }^{\mathrm{b}}$ & & & $\chi(2)=6.93$ & $.031 *$ & \\
\hline Single $(\mathrm{n}=659)$ & $618(19.0)$ & $41(17.4)$ & & & .535 \\
\hline Divorced/Widowed $(n=431)$ & $389(12.0)$ & $42(17.8)$ & & & .009 \\
\hline Married $(n=2397)$ & $2244(69.0)$ & $153(64.8)$ & & & .180 \\
\hline \multicolumn{6}{|l|}{ Children $<18$ living in the home } \\
\hline Yes $(n=2303)$ & $2142(65.1)$ & $161(67.6)$ & $\chi(1)=.61$ & .434 & \\
\hline
\end{tabular}


Table 3 (continued).

\section{Educational Status}

No college $(n=790)$

$732(22.7)$

$58(24.8)$

$\chi(3)=1.67$

.643

Some college $(n=824)$

$776(24.1) \quad 48(20.5)$

College graduate $(n=1063)$

$989(30.7) \quad 74(31.6)$

Graduate school $(n=778)$

$724(22.5)$

$54(23.1)$

Total Household Income

$\$ 0-39,999(n=538)$

489 (18.7)

$49(27.1)$

$\$ 40,000-59,999(\mathrm{n}=462)$

$432(16.5)$

$30(16.6)$

$\$ 60,000-79,999(n=438)$

$413(15.8)$

25 (13.8)

$\$ 80,000-99,999(\mathrm{n}=411)$

$384(14.7)$

$27(14.9)$

$\$ 100,000-119,999(\mathrm{n}=319)$

$301(11.5)$

$\$ 120,000$ or greater $(n=634)$

$602(23.0)$

$18(9.9)$

$32(17.7)$

\section{Employment Status ${ }^{\mathrm{c}}$}

Full-time, work or student $(\mathrm{n}=1852)$

$1775(64.1)$

$97(49.2)$

Part-time $(\mathrm{n}=350)$

Not employed $(n=326)$

Retired $(n=407)$

\section{First Primary Cancer Diagnosis ${ }^{\mathrm{d}}$}

Breast $(n=1003)$

Testicular $(n=306)$

Colorectal $(n=207)$

Hematological $(n=386)$

Prostate $(\mathrm{n}=251)$

Other $(\mathrm{n}=1385)$

$317(11.6) \quad 33(16.8)$

$300(11.0) \quad 26(13.2)$

$366(13.4) \quad 41(20.8)$

$950(28.9)$

$296(9.0)$

$185(5.6)$

$358(10.9)$

$237(7.2)$

1265 (38.4)

$43(18.1)$

$10(4.2)$

$22(9.3)$

$28(11.8)$

$14(5.9)$

Second Primary Cancer Diagnosis ${ }^{\mathrm{e}}$

$120(50.4)^{\dagger}$

$$
\chi(5)=9.12 \quad .105
$$

$$
\chi(3)=18.73 \quad<.001 *
$$

$<.001 *$

.031

.332

$.004 *$

$\chi(5)=28.97 \quad<.001 *$

$<.001 *$

.012

.020

.653

.453

$<.001 *$

$33(13.9)$

$32(13.4)$

$22(9.2)$

$16(6.7)$

$15(6.3)$

$13(5.5)$

Prostate

Time Since Last Treatment, years

$<1(\mathrm{n}=1041)$

$974(31.3)$

$67(30.0)$

$1219(39.1) \quad 94(42.2)$

$1-4(\mathrm{n}=1313)$

$538(17.3)$

34 (15.2)

$\geq 10(\mathrm{n}=411)$

$383(12.3)$

$28(12.6)$

Stage of Survivorship ${ }^{\mathrm{f}}$

Prefer not to answer/unsure $(n=47)$

Currently on treatment $(n=377)$

$44(1.3)$

$3(1.3)$

$356(10.8)$

$21(8.8)$

$136(4.1)$

$33(13.9)$

Less than 1 year post-treatment $(n=719)$

$676(20.5)$

43 (18.1)

$69(29.0)$

$1137(34.6)$

$69(29.0)$

$$
\chi(3)=1.09
$$

.779

$1-5$ years post-treatment $(n=1206)$

$941(28.6)$

Cancer Treatment

No chemotherapy $(n=1428)$

$1340(40.7)$

$88(37.0)$

$325(9.9)$

$21(8.8)$

Chemotherapy plus surgery and/or radiation

$1630(49.5)$

$129(54.2)$

$(\mathrm{n}=1759)$ 
Table 3 (continued).

Notes. Frequencies and percentages represent all available data for given variables; ${ }^{*}$ Statistical significance, $\mathrm{p}<.05 ;$ a Reported statistics are Pearson Chi-Square and independent sample t-tests; ${ }^{\text {-e }}$ post-hoc contingency table analyses using Pearson Chi-Square to detect within group differences; Bonferroni adjusted $p$-value thresholds to correct for Type I error are as follows: ${ }^{\mathrm{b}}$ partner status $p=.008$, ${ }^{c}$ employment status $p=.006,{ }^{\mathrm{d}}$ first primary cancer diagnosis $p=.004$, and ${ }^{\mathrm{f}}$ stage of survivorship $p=.004$.

${ }^{e}$ Only second primary cancer diagnoses that represented $\geq 5 \%$ of the MPC sample are reported.

${ }^{\dagger}$ Most frequent first primary cancer diagnoses represented in the other category for MPC survivors included: ovarian 14 (5.9\%); uterine 14 (5.9\%); and thyroid 10 (4.2\%). Remainder of other category diagnoses for MPC survivors not represented here included diagnoses with less than 10 respondents per diagnosis. 
Mean scores for primary outcomes by single and MPC groups are displayed in Table 4.

Table 4.

Mean Sum Scores on Outcome Category Scales by Single and Multiple Primary Cancer Groups

\begin{tabular}{|c|c|c|c|}
\hline & & $\begin{array}{c}\text { Single } \\
\text { Cancer } \\
\mathrm{n}=3295\end{array}$ & $\begin{array}{c}\text { Multiple } \\
\text { Primary } \\
\text { Cancers } \\
n=238\end{array}$ \\
\hline Category (number of respondents) & Range & \multicolumn{2}{|c|}{ Mean Score (SD) } \\
\hline $\begin{array}{l}\text { Psychological Distresses }(n=3028) \\
\text { Health Behaviors: }\end{array}$ & $0-8$ & $3.6(2.5)$ & $3.9(2.5)$ \\
\hline Healthy Lifestyle $(n=2723)$ & $0-4$ & $3.3(1.0)$ & $3.3(1.0)$ \\
\hline Positive Healthcare Utilization $(n=2739)$ & $0-3$ & $2.4(0.8)$ & $2.7(0.6)$ \\
\hline Benefit Finding $(n=3383)$ & $0-6$ & $4.6(1.6)$ & $4.6(1.5)$ \\
\hline
\end{tabular}

Multivariate linear regression analysis results are presented in Table 5. The final psychological distress model accounted for $8 \%$ of the model variance, $F(35,2670)=7.51$, $p<.001$. Significant predictors of psychological distress in the final model included age at survey, gender (female), partner status (divorced or widowed and married), first primary cancer diagnosis (colorectal), stage of survivorship (living with cancer as a chronic illness), and survivorship of MPCs. The final healthy lifestyle behaviors model accounted for $1 \%$ of the model variance, $F(35,2378)=1.88, p=.001$. Significant predictors of healthy lifestyle in the final model included race (nonwhite), total household income $(\$ 80,000-\$ 99,999$ and $\$ 100,000$ $\$ 119,999)$, employment status (not employed), time since last treatment (5-9 years), and stage of survivorship (greater than 5 years post-treatment). The final positive healthcare utilization behaviors model accounted for $4 \%$ of the variance in healthcare utilization, $F(35,2392)=3.80$, $p<.001$. Significant predictors of healthcare utilization in the final model included educational status (college graduate), first primary cancer diagnosis (colorectal and prostate), cancer treatment (chemotherapy plus surgery and/or radiation), and survivorship of MPCs. Lastly, the final benefit finding model accounted for $3 \%$ of the model variance, $F(35,2958)=3.38, p<.001$. 
Significant predictors of benefit finding in the final model included race (nonwhite), partner status (divorced or widowed and married), having children under 18 living in the home, total household income ( $\$ 100,000$ to $\$ 119,999)$ ), employment status (not employed), and cancer treatment (chemotherapy only and chemotherapy plus surgery and/or radiation). Survivorship of MPCs, our primary predictor variable of interest, was significantly associated with psychological distress (standardized $\beta=.046, p=.021$ ) and positive healthcare utilization behavior models (standardized $\beta=2.899, p=.004$ ) but not with healthy lifestyle behaviors (standardized $\beta=$ $.012, \mathrm{p}=.585$ ) or benefit finding (standardized $\beta=.011, \mathrm{p}=.562$ ). 
Table 5.

Multivariate Linear Regression Models of Psychological Distress, Healthy Lifestyle, Positive Healthcare Utilization, and Benefit Finding "As a Results of Having Cancer"

\begin{tabular}{|c|c|c|c|c|c|c|c|c|}
\hline \multirow[b]{2}{*}{$\begin{array}{l}\text { Sociodemographic or Clinical } \\
\text { Variables }\end{array}$} & \multicolumn{2}{|c|}{$\begin{array}{l}\text { Psychological Distress; } \\
\text { Model Adjusted } R^{2}=.08 ; \\
F(35,2640)=7.51, p<.001^{*}\end{array}$} & \multicolumn{2}{|c|}{$\begin{array}{c}\text { Healthy Lifestyle; } \\
\text { Model Adjusted } R^{2}=.01 \\
F(35,2378)=1.88, p=.001^{*}\end{array}$} & \multicolumn{2}{|c|}{$\begin{array}{l}\text { Healthcare Utilization; } \\
\text { Model Adjusted } R^{2}=.04 \\
F(35,2392)=3.80, p<.001^{*}\end{array}$} & \multicolumn{2}{|c|}{$\begin{array}{c}\text { Benefit Finding; } \\
\text { Model Adjusted } R^{2}=.03 ; \\
F(35,2958)=3.38, p<.001^{*}\end{array}$} \\
\hline & Standardized $\beta$ & $p$-value & Standardized $\beta$ & $p$-value & Standardized $\beta$ & $p$-value & Standardized $\beta$ & $p$-value \\
\hline Age at Survey, years ${ }^{a}$ & -.195 & $.012^{*}$ & .005 & .963 & .064 & .512 & -.007 & .929 \\
\hline $\begin{array}{l}\text { Age at Initial Diagnosis, years } \\
\text { Time since First Diagnosis, years }{ }^{a}\end{array}$ & $\begin{array}{l}-.017 \\
-.069\end{array}$ & $\begin{array}{l}.838 \\
.105\end{array}$ & $\begin{array}{l}.048 \\
.055\end{array}$ & $\begin{array}{l}.651 \\
.381\end{array}$ & $\begin{array}{l}.079 \\
-.008\end{array}$ & .447 & $\begin{array}{r}-.066 \\
.019\end{array}$ & $\begin{array}{l}.394 \\
.637\end{array}$ \\
\hline $\begin{array}{l}\text { Gender } \\
\text { Male } \\
\text { Female }\end{array}$ & $\begin{array}{l}\text { reference } \\
.171\end{array}$ & $<.001^{*}$ & $\begin{array}{l}\text { reference } \\
\quad .005\end{array}$ & -865 & $\begin{array}{l}\text { reference } \\
.010\end{array}$ & $\begin{array}{c}- \\
.722\end{array}$ & -.007 & .775 \\
\hline Race & & -.001 & .005 & . & .010 & $.12<$ & & \\
\hline $\begin{array}{l}\text { White } \\
\text { Other }\end{array}$ & $\begin{array}{l}\text { reference } \\
-.004\end{array}$ & .816 & $\begin{array}{l}\text { reference } \\
\quad .057\end{array}$ & $.006^{*}$ & $\begin{array}{l}\text { reference } \\
-.006\end{array}$ & .749 & $\begin{array}{l}\text { reference } \\
\quad .053\end{array}$ & $.003^{-}$ \\
\hline Partner Status & & & & & & & & \\
\hline $\begin{array}{l}\text { Single } \\
\text { Divorced/Widowed } \\
\text { Married }\end{array}$ & $\begin{array}{l}\text { reference } \\
.075 \\
.054\end{array}$ & $\begin{array}{l}- \\
.002^{*} \\
.045^{*}\end{array}$ & $\begin{array}{l}\text { reference } \\
\quad-.016 \\
-.004\end{array}$ & $\begin{array}{c}- \\
.554 \\
.889\end{array}$ & $\begin{array}{l}\text { reference } \\
-.046 \\
.006\end{array}$ & $\begin{array}{l}- \\
.077 \\
.827\end{array}$ & $\begin{array}{l}\text { reference } \\
-.051 \\
-.055\end{array}$ & $\begin{array}{c}- \\
.030^{*} \\
.033^{*}\end{array}$ \\
\hline Children $<18$ living in the home & & & & & & & & \\
\hline $\begin{array}{l}\text { No } \\
\text { Yes }\end{array}$ & reference & - & reference & - & reference & - & reference & - \\
\hline & .034 & .138 & -.016 & .551 & .010 & 669 & .114 & $<.001^{*}$ \\
\hline $\begin{array}{l}\text { Educational Status } \\
\text { No College } \\
\text { Some College } \\
\text { College Graduate } \\
\text { Graduate School }\end{array}$ & $\begin{array}{c}\text { reference } \\
-.014 \\
.009 \\
-.023\end{array}$ & $\begin{array}{l}- \\
.550 \\
.707 \\
.328\end{array}$ & $\begin{array}{l}\text { reference } \\
.018 \\
.029 \\
.035\end{array}$ & $\begin{array}{l}- \\
.472 \\
.274 \\
.175\end{array}$ & $\begin{array}{l}\text { reference } \\
-.010 \\
.068 \\
.042\end{array}$ & $\begin{array}{c}- \\
.690 \\
.009^{*} \\
.097\end{array}$ & $\begin{array}{c}\text { reference } \\
.013 \\
-.034 \\
-.031\end{array}$ & $\begin{array}{l}- \\
.559 \\
.142 \\
.187\end{array}$ \\
\hline Total Household Income & & & & & & & & \\
\hline$\$ 0-39,999$ & reference & - & reference & - & reference & - & reference & - \\
\hline$\$ 40,000-59,999$ & $<.005$ & .800 & .000 & .994 & -.041 & .065 & -.019 & .333 \\
\hline$\$ 60,000-79,999$ & -.008 & .693 & .020 & .371 & .031 & 169 & -.037 & .066 \\
\hline$\$ 80,000-99,999$ & -.573 & .567 & .050 & $.031^{*}$ & -.016 & .487 & -.034 & .093 \\
\hline$\$ 100,000-119,999$ & -.025 & .226 & .060 & $.009^{*}$ & .036 & .113 & .043 & $.035^{*}$ \\
\hline$\$ 120,000$ or greater & -.022 & .330 & .041 & .102 & .011 & .647 & .012 & .574 \\
\hline Employment Status & & & & & & & & \\
\hline Full-time & reference & - & reference & - & reference & - & reference & - \\
\hline Part-time & .006 & .848 & .003 & .885 & .020 & 337 & -.002 & .909 \\
\hline Not employed & .032 & .772 & -.053 & $.013^{*}$ & -.038 & .067 & -.061 & $.001^{*}$ \\
\hline Retired & .042 & .108 & .004 & .850 & .007 & .771 & -.004 & .859 \\
\hline
\end{tabular}


Table 5 (continued).

First Primary Cancer Diagnosis ${ }^{a}$

Breast

Testicular

Colorectal

Hematological

Prostate

Other

Time since Last Treatment, years $<1$

$1-4$
$5-9$

$\geq 10$

Stage of Survivorship

Prefer not to answer/unsure

Currently on treatment

Living with cancer as a chronic

illness

Less than 1 year post-treatment

1-5 years post-treatment

Greater than 5 years post-treatment

Cancer Treatment

No chemotherapy

Chemotherapy only

radiation

\begin{tabular}{|c|c|c|c|c|c|c|c|c|}
\hline $\begin{array}{l}\text { Survivorship of Multiple Primary } \\
\text { Cancers }\end{array}$ & .046 & $.021^{*}$ & -.012 & .585 & 2.899 & $.004^{*}$ & .011 & .562 \\
\hline
\end{tabular}

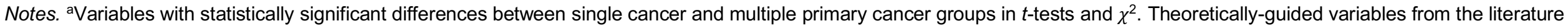
were determined a. priori and were also included in the multivariate model. *Statistical significance, $p<.05$. 


\subsubsection{Discussion}

Most striking in this study was the association between MPC and psychological distress, which was consistent with our recent review of literature (Belcher et al., 2016). Consistent with findings in a cohort of MPC survivors 10-20 years older than the MPC survivors in our sample (Gotay et al., 2007; Thong et al., 2013), survivorship of MPC did not predict benefit finding as a result of one's cancer experience. An unexpected finding unique to this study was that MPC survivors were more likely than single cancer survivors to report "living with cancer as a chronic illness" when asked to identify their stage of survivorship. This finding may indicate that MPC survivors face additional survivorship needs related to chronic illness and warrants additional study. Living in a state of chronic illness may be contributing to chronic stress and increasing risk for physical and psychological disease in this population (Corbin \& Strauss, 1988; Dowrick, Dixon-Woods, Holman, \& Weinman, 2005; Grady \& Gough, 2014; Miller et al., 2002).

Consistent with other MPC studies, we found that MPC survivors differed from single cancer survivors, in that they were older (Andrykowski, 2012; Thong et al., 2013) and had experienced more time since their initial cancer diagnosis (Burris \& Andrykowski, 2011). However, the MPC survivors represented by this LIVESTRONG cancer survivor sample were, on average, approximately 11-18 years younger than those currently represented in previous MPC literature (Andrykowski, 2012; Burris \& Andrykowski, 2011; Gotay et al., 2007; Thong et al., 2013). Additionally, MPC and single cancer survivors in this sample also differed by type of initial cancer diagnosis, with MPC survivors being less likely to have had breast cancer as their first diagnosis and more likely to fit into the "other" category (i.e., ovarian, uterine, and thyroid cancer). With differing cancer types come differing treatments and cancer treatment experiences. Thus, additional research is needed to determine the complex implications of differing diagnoses and treatments on health outcomes in MPC survivors.

While this study did not find statistical differences for income between groups, MPC survivors were less likely to be employed full time and more likely to be retired. Other 
preliminary work by our team has found that MPC survivors with recurrent ovarian cancer were more likely to endorse lower income and difficulty meeting basic needs than survivors with recurrent ovarian cancer "only" (Belcher et al., 2015). Another study found that MPC survivors experienced greater levels of lost productivity (e.g., employment) as compared to individuals without cancer and to survivors of single cancers (Dowling et al., 2013). With respect to partner status, we found that being divorced or widowed was more common in MPC survivors, though post-hoc testing with Bonferroni adjustments for Type I error did not identify statistical differences. Partner status, both divorced or widowed and married, was predictive of psychological stress. A study from the Netherlands found that MPC survivors report greater cancer impact on life, including body changes and interference with social activities (Thong et al., 2013). When viewed in context with findings from previous studies, results from this study support further examination of the impact of MPC on work and social role function in future MPC studies.

MPC survivors were more likely to report positive healthcare utilization, including engagement in cancer screenings and regular medical appointments. Similarly, Thong and colleagues (Thong et al., 2013) found that MPC presence was associated with greater health awareness. Conversely, MPC status was not associated with healthy lifestyle behaviors such as diet and regular exercise, which was consistent with Burris and Andrykowski's findings that those with MPC were more likely than single cancer survivors to have unhealthy behaviors (i.e., physical inactivity, smoking, and alcohol use). This may reflect a maladaptive behavioral coping response and warrants additional study in MPC survivors. As day-to-day chronic disease management responsibility shifts from providers to individuals (Barlow et al., 2002; Ryan, 2009), interventions to support survivors in initiating and maintaining healthy behaviors will be increasingly important in limiting exacerbation of existing conditions and preventing new conditions. 
Weaknesses in this study are acknowledged. Given the cross-sectional design, causal or temporal relationships between variables cannot be determined. Secondary analysis is limited to questions posed in the dataset, and information about psychological distress severity was not collected. Although we could account for $8 \%$ of variance in psychological distress in this large sample of cancer survivors, this suggests that there are other important factors that were not able to be included in this secondary data analysis, such as comorbidities, symptoms, physical function, perceived stress, social support and coping resources, self-management behaviors, financial toxicity, and biological stress responses. Additionally, MPC survivors represented $5.7 \%$ of our sample, which is slightly less than the $8 \%$ MPC representation that is typically found in the overall cancer survivor population (Mariotto, Rowland, Ries, Scoppa, \& Feuer, 2007). By conservatively excluding cases in which survivors reported a common site of metastasis as their second cancer, it is possible that we may have excluded true MPC cases from our analyses. Also, it has previously been reported that LIVESTRONG respondents are younger, less diverse, more educated, and wealthier than would be expected, which may be due to the voluntary, online nature of this survey (Low et al., 2014; Rechis et al., 2011) and may lead to decreased generalizability to the general cancer survivor population. However, this study expands what is currently known about MPC survivors by capturing a sample of survivors at an earlier age than has previously been described. Lastly, missing data, mostly in health behavior outcomes, may bias findings. Because rates of missingness were similar for variables between groups, we included as many cases as possible for both groups and presented all available data.

Strengths of this study include the ability to capture a large sample of post-treatment MPC survivors, to provide data on a younger demographic of MPC survivors than has previously been reported, the use of both negative (psychological distress) and positive (benefitfinding and health promotion behaviors) responses as independent outcomes, and models adjusted for a wide range of potential confounding variables. 


\subsubsection{Implications for Nursing}

Nurses are uniquely positioned to support unmet needs in MPC survivors. Nurses should be aware that survivorship needs may differ in cancer survivors based upon number of previous cancer diagnoses, and that the survivorship experience may differ between MPC and single cancer survivors. Additionally, MPC survivors are at an increased risk for psychological distress and may have additional needs related to living with cancer as a chronic illness (e.g., engaging in positive self-management behaviors such as healthy diet and exercise). Targeted and ongoing screening for distress in MPC survivors is warranted in specialty and/or primary care settings and may promote early identification and treatment to reduce potential negative downstream health effects.

Oncology nurse scientists should contribute to building the science in this area to identify, understand, and address the unique needs of MPC survivors. As the number of cancer survivors diagnosed with MPC grows, number of primary cancer diagnoses should be considered in study designs. While an early body of literature has begun to describe the prevalence of health outcomes in MPC survivors, a paucity of research exists surrounding mechanisms and risk factors for late and long-term effects of cancer and their potentially unique needs. Also unclear is whether the potential for care silos and lack of a clinical home influences health outcomes in MPC survivors. Nurses are well suited to study, assess, and address MPC care needs.

\subsubsection{Conclusions}

Cancer survivors are increasingly being diagnosed with additional subsequent primary cancers. Our findings provide additional evidence that MPC survivors differ from their single cancer counterparts and are at increased risk for psychological distress. Our findings support a need to specifically identify, understand, and address the ongoing, unique needs of MPC survivors. Additional research is needed to identify MPC survivors most at risk for poor outcomes and to 
understand the care needs and mechanisms that contribute to poor health outcomes in this growing cancer survivor population.

\subsubsection{Acknowledgements}

Portions of this study have been presented in poster and abstract forms at the $8^{\text {th }}$ Biennial Cancer Survivorship Research Conference, Washington, D.C, on June 16, 2016

This research was supported in part by the University of Pittsburgh School of Nursing (Newmeyer-Thompson Doctoral Student Research Award, Belcher); the Robert Wood Johnson

Foundation (Future of Nursing Scholars, Belcher); the National Cancer Institute (K32CA149082, Posluszny); the National Cancer Institute (P30CA047904, University of Pittsburgh Cancer Institute Cancer Center); and the LIVESTRONG Foundation (source data). 


\subsection{DISSERTATION MANUSCRIPT 3: DISSERTATION AIMS 1 AND 2}

\section{Adapting a Psychobehavioral Stress-Response Model to Characterize Risks in Survivors of Multiple Primary Cancers}

\subsubsection{Abstract}

Objective: The purpose of this study was to evaluate hypothesized relationships in an adapted psychobehavioral stress-response model among adults with multiple primary cancers (MPC). We aimed to 1) test the hypothesized model to examine associations among measured latent variables: perceived stress, psychological and behavioral responses, financial toxicity, and social role and physical health and 2) explore associations between individual characteristics and latent variables in the model.

Methods: This cross-sectional study of MPC survivors included participants whose first cancers (stages I-III) were diagnosed within 1-10 years. Participants were recruited through a regional tumor registry. Participants completed a battery of valid questionnaires to measure latent variables and covariates; data were extracted from tumor registry and medical record data. Structural equation modeling (SEM) was performed to fit and modify the measurement model, specify the full SEM, and identify significant predictors.

Results: 211 participants completed surveys. Data fit a modified four-factor measurement model with latent variables including self-management, distress, financial toxicity, and functional health. Overweight BMI, graduate level education, less neuroticism, and increased social support predicted better self-management. Poorer self-management, greater neuroticism, and lower social support predicted increased distress. Greater distress predicted financial toxicity. Greater distress and financial toxicity predicted poorer functional health.

Conclusions: Self-management behaviors and distress are modifiable targets with potential to mitigate financial toxicity and improve functional health. MPC survivors with extreme BMls, less education, greater neuroticism, and lower social support should be considered at risk for poorer self-management and negative downstream health outcomes. 
Keywords: multiple primary cancers; subsequent primary cancers; cancer survivorship;

biobehavioral oncology; health behaviors; self-management; distress; financial toxicity; health outcomes; conceptual model 


\subsubsection{Introduction}

As the population ages and cancer survival improves, the incidence of subsequent, or multiple, primary cancer (MPC) diagnoses is also increasing. Three million, or one in five, U.S. cancer survivors are diagnosed with an additional cancer in their lifetime (Morton et al., 2014), which increases to one in four for cancer survivors over 65 years old (Murphy et al., 2017). MPCs are histologically distinct cancers that have been ruled out as metastatic disease (Begg, 1999) and are a leading cause of morbidity and mortality among cancer survivors. Risk for psychological distress, risky health behaviors, and poorer physical health is greater in MPC survivors than in single- and no-cancer controls (Andrykowski, 2012; Belcher et al., 2017; Belcher et al., 2016; Burris \& Andrykowski, 2011; Dowling et al., 2013; Gotay et al., 2007; Thong et al., 2013). Additionally, there is evidence to suggest that MPC survivors may be at increased risk for financial toxicity and impaired social functioning (Belcher et al., 2015; Dowling et al., 2013; Thong et al., 2013).

An adapted psychobehavioral stress model was used to identify pathways linking stress to poor health outcomes. Perceived stress is the perception that one's demands exceed his or her coping resources (Lazarus \& Folkman, 1984), which, if left uninterrupted, can result in a cascade of psychological, behavioral, and biologic responses that negatively impact health outcomes. Links between perceived stress, psychological responses (e.g., depression, anxiety) and behavioral responses (e.g., positive self-management and risky health behaviors), and physical health outcomes (e.g., physical function, symptoms, and comorbidities) have been well established (Andersen et al., 1994; Andersen et al., 2008; Bower \& Lamkin, 2013; Cohen et al., 1995; Sherwood et al., 2008).

Additional pathways (see Figure 6) impacting financial and social outcomes are also important in this adapted model. Financial toxicity is increasingly recognized as a potential consequence of cancer and cancer treatment (Zafar \& Abernethy, 2013; National Cancer Institute, n.d.-a). and has been linked to poor treatment adherence and mortality (Kent et al., 
2013; National Cancer Institute, 2018c; Park \& Look, 2018; Ramsey et al., 2016;). MPC is a known risk factor for financial toxicity (Belcher et al., 2015; Dowling et al., 2013; National Cancer Institute, 2018c). Cancer is also known to impact social function (i.e., involvement in and satisfaction with one's usual social relationships and activities) (Bode et al., 2010; Costa, Mercieca-Bebber, Rutherford, Gabb, \& King, 2016; Hahn, Cella, Bode, \& Hanrahan, 2010; Syrjala \& Yi, 2018). Having MPC has been associated with interference in a range of life and social role activities (e.g., availability to family, not being understood, impact on daily activities) and limitations in ability to do work (Dowling et al., 2013; Thong et al., 2013).

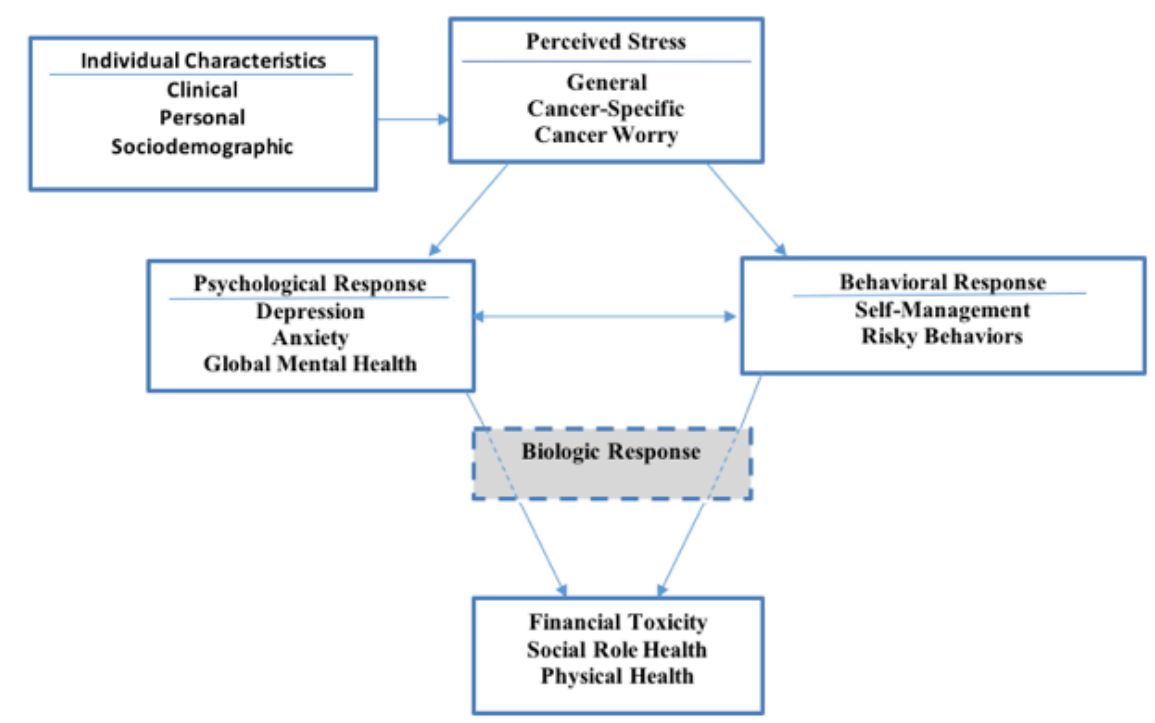

Figure 6. Conceptual model. This figure depicts the cascade of psychological, behavioral, and biologic responses to perceived stress that can negatively impact health outcomes in adults with multiple primary cancers. Biologic responses will be investigated in future studies.

Despite the rising prevalence of MPC and associated poor health outcomes, there is a lack of research examining potential pathways linking MPC to negative outcomes, and research in this patient population has lacked conceptual models to guide inquiry. The purpose of this study, the first to evaluate hypothesized relationships within an adapted psychobehavioral stress-response model in MPC, is to 1) identify important factors associated with negative 
health outcomes and 2) guide early identification of MPC survivors at risk for poor health outcomes.

Specifically, the primary aim was to test the hypothesized model using structural equation modeling to examine the linear relations among the measured latent variables of perceived stress, psychological response, behavioral response, financial toxicity, social role function, and functional health. It was hypothesized that:

1. Perceived stress has direct effects on a) psychological response, b) behavioral response, c) financial toxicity, d) social role function, and e) physical health;

2. Behavioral response has direct effects on a) psychological response, b) financial toxicity, c) social role function, and d) physical health; and

3. Psychological response has direct effects on a) financial toxicity, b) social role function, and c) physical health.

The secondary aim was to explore individual, sociodemographic, and clinical characteristics that may directly impact upstream latent variables in the model.

\subsubsection{Methods}

3.3.3.1 Sample and setting. An honest broker system was used to recruit participants through the UPMC Cancer Network Registry, a member of the National Program of Cancer Registries who report to the larger Pennsylvania Cancer Registry (“Cancer registry requirements," n.d.). Study eligibility criteria included a history of two primary cancers based on Surveillance, Epidemiology, and End Results (SEER) Program coding rules (Johnson et al., 2007), with first cancer sites consistent with most prevalent first diagnoses experienced by adults living after MPC diagnoses: female breast, prostate, colorectal, urinary bladder, uterine, melanoma, kidney and renal pelvis, lung and bronchus, oral cavity and pharynx, thyroid, or ovary (American Cancer Society, 2009). Additional query criteria included first diagnoses 1-10 years ago; stage IIII cancers at time of diagnosis (both first and second primary cancers); and able to read and complete questionnaires in English. Cases of non-melanoma skin cancer; in situ cancer; 
advanced staging (i.e., stage IV) at diagnosis, and recurrent cancers were excluded from the query. Additional study exclusion criteria included being listed in the corporate opt-out database and being deemed not appropriate for contact based on physician champion review of cases. Sampling was conducted using stratified systematic sampling by disease type with oversampling of individuals from traditionally underrepresented races and ethnicities.

3.3.3.2 Study procedures. Participants were recruited, and cross-sectional data were collected using a series of contacts based on a modified version of Dillman's Tailored Survey Method (Dillman et al., 2014). The honest broker system was used to identify prospective participants and invite study participation. Letters of invitation were signed by physician champions, and follow up reminder postcards were mailed to individuals who did not respond to initial contact. Participants could either complete the survey online via Qualtrics, an Institutional Review Board (IRB) approved, Web-based survey system (University of Pittsburgh Computing Services and Systems Development, n.d.), or request a postage-paid paper survey via mail, email, or telephone. Informed consent was obtained based on mode of selected survey delivery. Partially completed online surveys prompted reminder emails on Days 5,10 , and 21 following survey invitations. For study packets that had not been returned, reminder postcards were sent on Day 14, and additional follow-up contacts were made via participant-initiated mode of contact on Day 21. Following survey completion, participants were sent a thank you card or email message and compensated with a five-dollar Amazon code. Individuals who did not wish to participate were asked to complete a voluntary, anonymous refusal form, either online or on paper, that included five basic sociodemographic questions. Key clinical variables were obtained from both the Cancer Registry database and medical record review. This study was approved by the University of Pittsburgh IRB.

3.3.3.3 Instruments. A battery of valid and reliable measures was administered to operationalize model latent variables. Short forms and common measures were administered when possible to decrease participant burden and increase generalizability. PROMIS measures 
were scored using individual participant response pattern scoring through the online Assessment Center Scoring Service, ${ }^{\mathrm{SM}}$ for resulting T-scores, 50=average, while T-score=60 is one standard deviation higher, or better, than average (Cella, Gershon, Bass, \& Rothrock, 2017). When no information was available regarding number of items required to scale a score, item scores were imputed if at least $80 \%$ of scale items were completed.

\subsection{Sociodemographic, personal, and clinical predictors.}

Sociodemographic. An adapted self-reported instrument (Sereika \& Engberg, 2006) was administered to assess sociodemographic variables (e.g., age, gender, race, partner status, education, and employment).

Personality. The neuroticism and conscientiousness domains of the International Personality Item Pool (IPIP), mini, were administered to assess personality (Donnellan et al., 2006; Goldberg, 1999). Each 4-item subscale is scored 1 (very accurate) to 5 (very inaccurate), with higher sum scores indicating greater neuroticism and conscientiousness.

Social support. The 12-item Interpersonal Support Evaluation List (ISEL-12) was used to measure perceived social support. Items, rated 1 (definitely true) to 4 (definitely false), are summed, with higher total scores indicating better perceived social support.

Clinical variables. First cancer site and time from first cancer diagnosis was obtained from the Cancer Registry. Second cancer site, time from second cancer diagnosis and treatment, time between cancer diagnoses, more than 2 primary cancer diagnoses, and body mass index (BMI) were extracted from the medical record.

\subsection{Latent variables.}

Perceived stress. Three measures were used to capture the latent variable of perceived stress. The 10-item Perceived Stress Scale (PSS) captures global perceived stress (Cohen et al., 1983; Golden-Kreutz et al., 2004) on a 0 (never) to 4 (very often) scale. The PSS total summary score ranges from 0 to 40 , where higher scores suggest greater perceived stress. The Revised Impact of Event Scale (IES-R) was used to assess cancer-specific stress (Salsman et 
al., 2015; Weiss, 2007; Weiss \& Marmar, 1997). The IES-R, with 22 items rated 0 (not at all) to 4 (extremely), produces a summary total score ranging from 0 to 88 , where higher scores indicate greater cancer-specific stress. The 3-item Cancer Worry subscale of the Assessment of Survivor Concerns scale was used to measure cancer survivor stress related to future tests, new diagnoses, and recurrences (Gotay \& Pagano, 2007; Thewes et al., 2012). Worry items are rated on a 4-point Likert scale, from 1 (not at all) to 4 (very much), and are summed to yield a summary score ranging from 3 to 12 . Higher cancer worry subscale scores are indicative of greater worry. In the current study, the combined measures of perceived stress demonstrated acceptable internal consistency (standardized Cronbach's $\alpha=.75$ ).

Psychological responses. Three measures were used to capture the psychological response latent variable. Two-item Personal Health Questionnaire (PHQ) depression and Generalized Anxiety Disorder (GAD) short form scales were used to measure depression and anxiety, respectively (Kroenke et al., 2003, 2007; Löwe, Kroenke, \& Gräfe, 2005; Plummer, Manea, Trepel, \& McMillan, 2016; Whooley, Avins, Miranda, \& Browner, 1997). Items from each scale, ranked 0 (not at all) to 3 (nearly every day), produce sum scores ranging from 0 to 6 ; scores $\geq 3$ should prompt further clinical assessment. The 10-item Global Mental Health subscale of the PROMIS Global Health measure (Hays et al., 2009; HealthMeasures, 2017), version 1.0, was used to assess global mental health. Raw scores were converted to v1.2 format and submitted to the Assessment Center Scoring Services website for cancer sample calibrated scoring as T-scores. Internal consistency of the combined psychological response measures in this sample was good (standardized Cronbach's $\alpha=.85$ ).

Behavioral responses. Three measures were used to capture the behavioral response latent variable. The 40-item Health Education Impact Questionnaire (hei-Q) (Elsworth et al., 2015; Maunsell et al., 2014; Osborne et al., 2007) assess eight domains of self-management behaviors including health-directed activities (HAD); positive and active engagement in life (PAE); emotional distress (ED); self-monitoring and insight (SMI); constructive attitudes and 
approaches (CAA); skill technique and acquisition (STA); social integration and support (SIS); and health service navigation (HSN). Items are ranked 1 (strongly disagree) to 4 (strongly agree), and are averaged to produce mean subscale scores, with higher scores indicating better self-management (except the emotional distress subscale, which is interpreted inversely). The PROMIS Alcohol Use short form 7a (Patient-Reported Outcomes Measurement Information System, 2014; Pilkonis et al., 2016) was administered to assess alcohol use. Respondents who reported alcohol use were administered six additional items, ranked on a 1 to 5 scale, with higher raw sum scores, calibrated against a chronic illness sample, indicating greater consumption (scale range 5-25; negative screens=0). The Cancer Patient Tobacco Use Questionnaire (CTUQ) was used to assess tobacco use (Land et al., 2016; National Cancer Institute, 2016), yielding data for calculation of pack-year-history. In the current study these combined measures of behavioral response demonstrated questionable internal consistency (standardized Cronbach's $\alpha=.67$ ); when alcohol and tobacco use were later dropped from multivariate analyses, acceptable internal consistency was achieved for the set of heiQ subscales (standardized Cronbach's $\alpha=.77$ ), leading to renaming the Behavioral Response latent variable as Self-Management Behaviors.

Financial toxicity. Two measures were used to capture the latent variable of financial toxicity. The first was the 11-item Comprehensive Score for financial Toxicity (COST), v1 (De Souza et al., 2016; De Souza et al., 2014). Items, ranked on a 5-point scale ranging from 0 (not at all) to 4 (very much), were summed to produce a total score, ranging from 0 to 44 , where higher total scores indicate better financial wellbeing. The second, the Economic Hardship Questionnaire (EHQ) (Barrera et al., 2001), includes four subscales: inability to make ends meet (MEM), not enough money for necessities (MFN), economic cutbacks and adjustments (EA), and anticipation of future financial strain (FS), with higher scores indicating greater hardship. The 2-item MEM and FS subscales include items ranked 1 to 5 and yield mean subscale scores. The first 4-items from the MFN subscale, ranked 1 (strongly agree) to 5 (strongly 
disagree), yield a sum score ranging from 4 to 20 . The 9-item EA subscale consists of a checklist of adjustments made due to financial need, with yes endorsements summed to produce a 0-9 subscale score. Internal consistency of the combined measures in this sample was excellent (standardized Cronbach's $\alpha=.92$ ).

Social role function. Two measures were used to capture the latent variable of social role function. The 8-item PROMIS Ability to Participate in Social Roles and Activities V2.0 and 8-item PROMIS Satisfaction with Participation in Social Roles short forms measure perceived ability to participate in social roles and activities and satisfaction with participation in usual roles and activities, respectively. Items are rated 5 (never) to 1 (always) and 1 (not at all) to 5 (very much) on the respective measures. Each measure was calibrated against the default social supplement sample yielding a T-score. Internal consistency in this sample was good (standardized Cronbach's $\alpha=.85$ ).

Physical health. Three measures were used to capture the physical health latent variable. The 10-item PROMIS Physical Function 10a short form assesses limitation in physical function (Cook et al., 2016; Patient-Reported Outcomes Measurement Information System, 2015; Rose et al., 2014). Items are ranked 1 to 5 and yield a T-score, calibrated against a cancer sample. The 19-item MD Anderson Symptom Inventory (MDASI) (Aktas et al., 2015; Cleeland et al., 2000; Cleeland, 2016) was used to assess symptom burden, a mean composite of the top five of 13 rated symptoms in the sample (range=0-10, with higher scores indicating greater burden), and symptom distress, the overall mean of the 6 interference items (possible range $=0-10$, with higher scores indicating greater interference). The brief 10 -item self-report comorbidity index was used to assess comorbidities (Charlson et al., 1987; Chaudhry et al., 2005). Weighted items were summed to create an index score, ranging from 0 to 25 , with higher scores indicating worse comorbidity. Internal consistency of the combined measures in this sample was good (standardized Cronbach's $\alpha=.86$ ). 
3.3.3.4 Data analyses. Data for 215 participants were analyzed using IBM ${ }^{\circledR}$ SPSS $^{\circledR}$ software, version 25 (IBM Corp., Armonk, NY) at significance level of .05 for two-sided hypothesis testing. Chi-square test of independence and independent sample t-tests for two-sided hypothesis testing were used to compare characteristics of respondents with nonrespondents. Descriptive statistics, frequency distributions, and exploratory analyses were performed to identify any data anomalies (i.e., normality, univariate and multivariate outliers, multicollinearity, missing data) and characterize the sample. Z-scores and Malahalanobis distance were assessed for univariate and multivariate outliers, respectively. Score alterations were applied to variables with extreme outliers (see Table 6 footnote). Reverse scoring was applied as necessary to achieve consistent direction among sets of measured variables in multivariate analyses. Due to scaling differences across measured variables, measured variables were re-scaled, such that variance of the measured variable was $<10$ to enhance model convergence. Confirmatory factor analyses were performed for groups of latent variables and the measurement model. Internal consistency of the sets of measured variable for each latent variable was evaluated using the standardized Cronbach's alpha $(\alpha)$ coefficient, where Cronbach's $\alpha>.7$ is considered acceptable (George \& Mallery, 2003).

SEM was performed in two stages. First, the measurement model was fit and modified. All of the measurement models were estimated employing robust maximum likelihood estimation to account for normality violations ( $\mathrm{Li}, 2016)$. Next, the full SEM was then specified using Mplus, version 8.1 (Muthén \& Muthén, Los Angeles, CA). Post-hoc model modifications were made to improve model fit and parsimony based on model test statistics, fit indices, and theory. The model chi-square test was evaluated for model fit. Additional practical fit indices were evaluated for model fit, and cut points were considered acceptable if: comparative fit index $(\mathrm{CFI}) \geq .95$; Tucker-Lewis Index $(\mathrm{TLI}) \geq .95$; standardized root mean residual $(\mathrm{SRMR}) \leq .08$; root mean square error of approximation (RMSEA) <.06 (Hu \& Bentler, 1999; Kenny, 2015; Little, 2013). Significance of standardized path coefficients was evaluated to determine paths to retain 
in the final modified model. For the original SEM with a sample size of 206 with 309 degrees of freedom, the analyses had $>.99$ power for either the test of a close fit (i.e., RMSEA $=.05$ vs RMSEA $_{A}=.08$ ) or exact fit (i.e., RMSEA $_{0}=0.0$ vs RMSEA $_{A}=.05$ ).

After model adequacy was determined, upstream paths were added to explore possible personal, sociodemographic, and clinical covariates for improved model fit and determination of significantly associated characteristics that may be used to identifying individuals at risk for poor downstream health outcomes.

\subsubsection{Results}

3.3.4.1 Sample statistics. The Cancer Registry identified 2233 people who were potentially eligible for study participation. Of these people, $62(2.8 \%)$ were on the corporate "do not contact" list, and physicians requested that 46 (2.1\%) not be contacted. After sampling, 1443 letters were mailed to the remaining potentially eligible individuals. Thirty-nine letters $(2.7 \%)$ had undeliverable mailing addresses, 11 (0.8\%) were deceased, and $2(0.1 \%)$ had advanced Alzheimer's disease/dementia. This analysis used only complete cases, thus, four were dropped due to partial online survey completion $(n=211)$. Of the remaining 1390 cases, $211(15.2 \%)$ completed surveys (125 [59.2\%] on paper; 86 [40.8\%] online). See Figure 7 for recruitment flowchart. Five cases were also identified as being multivariate outliers and were also dropped from analysis (final sample size for multivariate analysis, $n=206$ ). 


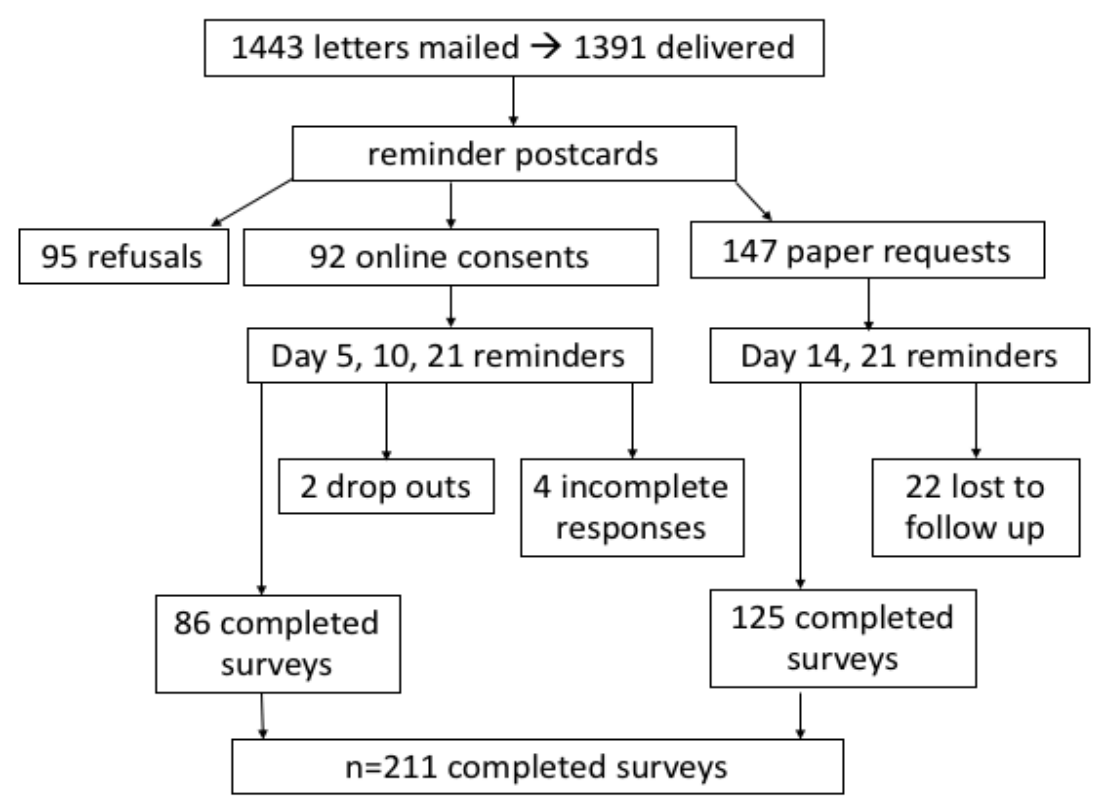

Figure 7. Study flow. This flowchart illustrates study recruitment.

Sociodemographic and clinical characteristics of 211 participants who completed the survey are summarized in Tables 6 and 7 (see also supplementary Tables 9-12). Participants were on average 67 years of age. The majority were female, white, married, retired, and insured. A broad range of educational levels was represented in the sample, with nearly $40 \%$ of participants having a high school level education or less. Over $30 \%$ of participants reported difficulty paying for basic needs. Neuroticism was in the lower quartile of the scale range, conscientiousness was in the upper quartile of the possible range (10 and 15 , respectively), and perceived social support was fairly high (39).

On average, approximately six years had passed since participants' first cancer diagnoses, and the most common sites of first cancer diagnoses included lung, prostate, breast, thyroid, and melanoma. Most commonly identified sites of second cancer diagnoses identified via medical record data included breast, lung, thyroid, kidney, and melanoma. Irrespective of sequence, breast and prostate cancer were the most commonly diagnosed cancers in this sample ( $n=47$ [22.3\%] and $n=45$ [21.3\%], respectively), followed by lung cancer ( $n=42$ [19.9\%]) (see supplementary Table 10 for complete list). Fifty percent of participants experienced their 
first and second cancer diagnoses within a six-month time period. An average of approximately four years had passed since participants' cancer treatment completion, and almost $16 \%$ of participants had more than two documented primary cancer diagnoses. The most common combinations of first and second cancer sites diagnosed in this sample were second primaries in the same site: lung/lung $(n=29,9.8 \%)$; breast/breast $(n=17,8.3 \%)$; thyroid/thyroid $(n=12$, $5.9 \%$ ); melanoma/melanoma $(\mathrm{n}=11,5.4 \%)$ (full list available in supplementary Tables 11 and 12). Over $40 \%$ of participants were classified as obese (BMI $\geq 35)$, based on most recent records.

\section{Table 6}

Self-Reported Sample Characteristics $(N=211)$

\begin{tabular}{|c|c|c|}
\hline \multirow[t]{2}{*}{$\underline{\text { Characteristics }}$} & \multicolumn{2}{|c|}{ Summary Statistics } \\
\hline & Mean (SD) & Range \\
\hline Current Age, years* $(n=211)$ & $67.1(11.4)$ & $29-89$ \\
\hline \multicolumn{3}{|l|}{ Personality } \\
\hline Neuroticism $(n=211)$ & $9.6(3.7)$ & $4-20$ \\
\hline Conscientiousness* $(n=211)$ & $15.1(3.2)$ & $6-20$ \\
\hline \multirow[t]{2}{*}{ Social Support } & $39.1(7.6)$ & $15-48$ \\
\hline & $\underline{\mathrm{n}}$ & $\underline{\%}$ \\
\hline \multicolumn{3}{|l|}{ Gender $(n=211)$} \\
\hline Female & 128 & 60.7 \\
\hline \multicolumn{3}{|l|}{ Race/Ethnicity $(n=211)$} \\
\hline White or Caucasian & 196 & 93.0 \\
\hline \multicolumn{3}{|l|}{ Partner Status $(n=211)$} \\
\hline Married/Living with Partner & 147 & 69.7 \\
\hline \multicolumn{3}{|l|}{ Educational Attainment $(n=209)$} \\
\hline High School/GED or less & 81 & 38.8 \\
\hline Beyond high school through bachelor's degree & 81 & 38.8 \\
\hline Master's Level or beyond & 47 & 22.5 \\
\hline \multicolumn{3}{|l|}{ Difficulty Paying for Basic Needs $(n=209)$} \\
\hline Not Difficult & 142 & 67.9 \\
\hline Somewhat or Extremely Difficult & 67 & 32.1 \\
\hline \multicolumn{3}{|l|}{ Employment Status $(n=209)$} \\
\hline Retired, Not Working & 109 & 52.2 \\
\hline Working Full-time ( $\geq 35$ hours/wk) & 39 & 18.7 \\
\hline Retired, Working Part- or Full-time & 18 & 8.6 \\
\hline Disabled/Unable to Work & 18 & 8.6 \\
\hline Full-time Homemaker & 12 & 5.7 \\
\hline Working Part-time (<35 hours/wk) & 10 & 4.8 \\
\hline Laid Off/Unemployed/Looking for Work & 3 & 1.4 \\
\hline Healthcare Insurance $(n=210)$ & 208 & 99.0 \\
\hline
\end{tabular}


Table 6 (continued).

Notes. SD = standard deviation.

*Values reflect score alterations applied to adjust for extreme values in multivariate analyses. 
Table 7.

Sample Clinical Characteristics $(N=211)$

\begin{tabular}{|c|c|}
\hline Characteristics & $\underline{\mathrm{n}(\%)}$ \\
\hline \multicolumn{2}{|l|}{ First Cancer Diagnosis Site, $(n=211)$} \\
\hline Lung / Bronchus & $33(15.6)$ \\
\hline Prostate & $30(14.2)$ \\
\hline Breast & 26 (12.3) \\
\hline Thyroid & $22(10.4)$ \\
\hline Melanoma & $21(10.0)$ \\
\hline Uterine Corpus (includes endometrial) & $20(9.5)$ \\
\hline Kidney / Renal Pelvis & $17(8.1)$ \\
\hline Colorectal & $15(7.1)$ \\
\hline Urinary Bladder & $12(5.7)$ \\
\hline Ovarian (includes fallopian tube) & $8(3.8)$ \\
\hline Oral Cavity / Pharynx & $7(3.3)$ \\
\hline Years from First Cancer Diagnosis, Mean (SD) $(n=211)$ & 5.8 (2.9); range: $0-11$ \\
\hline \multicolumn{2}{|l|}{ Most Frequent Second Cancer Diagnosis Sites* $(n=205)$} \\
\hline Breast & $38(18.5)$ \\
\hline Lung / Bronchus & $29(14.1)$ \\
\hline Thyroid & $19(9.3)$ \\
\hline Kidney / Renal Pelvis & $18(8.8)$ \\
\hline Melanoma & $16(7.8)$ \\
\hline \multicolumn{2}{|l|}{ Most Frequently Diagnosed Cancer Site Patterns (First } \\
\hline \multicolumn{2}{|l|}{ Cancer, Second Cancer $)^{*}(\mathrm{n}=205)$} \\
\hline Lung, Lung & $20(9.8)$ \\
\hline Breast, Breast & $17(8.3)$ \\
\hline Thyroid, Thyroid & $12(5.9)$ \\
\hline Melanoma, Melanoma & $11(5.4)$ \\
\hline Bladder, Prostate & $10(4.9)$ \\
\hline Years Since Second cancer Diagnosis, Mean (SD) $(n=197)$ & 4.0 (2.7); range: $0-10$ \\
\hline $\begin{array}{l}\text { Years Since Most Recent Cancer Treatment, Mean (SD) } \\
(n=204)\end{array}$ & 2.7 (2.9); range: $0-10$ \\
\hline $\begin{array}{l}\text { First and Second Cancers Diagnosed within } 6 \text { Month } \\
\text { Timeframe }(n=206)\end{array}$ & $102(49.5)$ \\
\hline $\begin{array}{l}\text { More than Two Cancer Diagnoses documented in medical } \\
\text { record }(n=211)\end{array}$ & $33(15.6)$ \\
\hline \multicolumn{2}{|l|}{ Most Recent BMI by Category $(n=209)$} \\
\hline$\leq 18.5$ (underweight) & $5(2.4)$ \\
\hline $18.5-24.9$ (normal) & $62(24.9)$ \\
\hline 25.0-29.9 (overweight) & $67(32.1)$ \\
\hline$\geq 30$ (obese) & $85(40.7)$ \\
\hline
\end{tabular}

Note. SD = standard deviation.

*See supplementary online material for complete list of second cancer diagnoses, frequencies of cancer sites diagnosed as either first or second cancer, and patterns of first and second cancer diagnoses. 
Ninety-five individuals who did not participate in the study (8.0\%) submitted anonymous refusal forms. While 18 of these individuals (18.9\%) did not provide a refusal reason, top listed reasons for not participating included perceived ineligibility (e.g., "I've only had one cancer," $n=20[21.1 \%])$, lack of time $(n=17[17.9 \%])$, advanced age $(n=12[12.6 \%])$, concern with accessing medical records $(n=10[10.5 \%])$, and health issues $(n=9[9.5 \%])$. Comparison of deidentified Cancer Registry data using independent sample t-tests and Pearson's chi-square tests of independence indicated that study participants were similar to non-respondents on all variables examined, including age, sex, race, marital status, primary payer at first diagnosis, years since first cancer diagnosis, and first cancer site (see supplementary online material, Table 13).

3.3.4.2 Description of key variables in the measurement model. Table 8 displays scores for all variables considered in the originally hypothesized measurement model.

Table 8.

Scores for Variables in the Originally Hypothesized Measurement Model $(N=211)$ Measures by Latent Variables

Perceived Stress

Perceived Stress, general $(n=206)$

Cancer-Specific Stress* $(n=210)$ Cancer worry $(n=211)$

Psychological Response Depression* $(n=211)$

Anxiety* $(n=209)$ Global Mental Health $(n=210)$

Behavioral Response Self-Management Health Directed Activity ( $n=208)$ Positive and Active Engagement in Life $(n=208)$ Emotional Distress ( $n=208)$ Self-Monitoring and Insight $(n=208)$ Constructive Attitudes and Approaches* $(n=208)$ Skill and Technique Acquisition* $(n=208)$ Social Integration and Support $(n=208)$ Health Service Navigation* $(n=208)$ Alcohol Use ${ }^{\infty}(n=105)$ Tobacco Use: Pack-Year History* $(n=205)$ $\underline{\text { Descriptive Statistics }}$ Mean (SD) $\underline{\text { Range }}$

$13.0(7.0)$

$14.7(13.0)$ $7.5(2.9)$

$1.0(1.4)$ $.9(1.3)$ $48.4(7.9)$ 
Table 8 (continued).

Financial Toxicity

Financial Toxicity $(n=205)$

Economic Hardship

Financial Strain* $(n=209)$

Inability to Make Ends Meet $(n=208)$

Not Enough for Necessities $(n=208)$

Cutbacks and Adjustments* $(n=209)$

Social Role Function

Social Role Function Ability* $(n=209)$

Social Role Function Satisfaction* $(n=210)$

Physical Health

Physical Function $^{\dagger}(\mathrm{n}=211)$

Symptoms

Symptom Burden $(n=209)$

Symptom Distress* $(n=209)$

$28.6(10.2)$

$0-44$

$1.2(.4)$

$2.0(1.1)$

$1.9(1.1)$

$.9(1.7)$

$1-2.3$

$1-5$

$1-5$

$52.3(10.3) \quad 25.9-65.4$

$50.7(10.9) \quad 26.5-65.5$

$46.7(8.3) \quad 29.1-55.2$

Comorbidity Index $(\mathrm{n}=211)$

Notes. SD=standard deviation.

$2.2(2.2) \quad 0-8.6$

$1.5(2.3) \quad 0-10$

4.8 (3.4) $\quad 0-15$

*Values reflect score alterations applied to adjust for extreme values in multivariate analyses.

${ }^{\dagger}$ Mean composite of top five most severely rated symptoms in sample (i.e., fatigue, drowsy, dry mouth, disturbed sleep, and pain).

$\infty$ Alcohol use scores can only be calculated for individuals who screen positive for consuming alcohol in the previous 30 days.

3.3.4.3 Measurement model. A confirmatory factor analysis was performed through Mplus. A six-factor model for stress, psychobehavioral responses, and health outcomes was originally hypothesized and is presented in Figure 8, where the factors or latent variables are represented by ovals, and measured variables are represented by rectangles. Absence of a line connecting variables implies no hypothesized direct effect. Indicators of factors for the six latent variables are described with instrument descriptions (see Section 3.3.3.3). All six factors were hypothesized to covary with one another in the measurement model. 


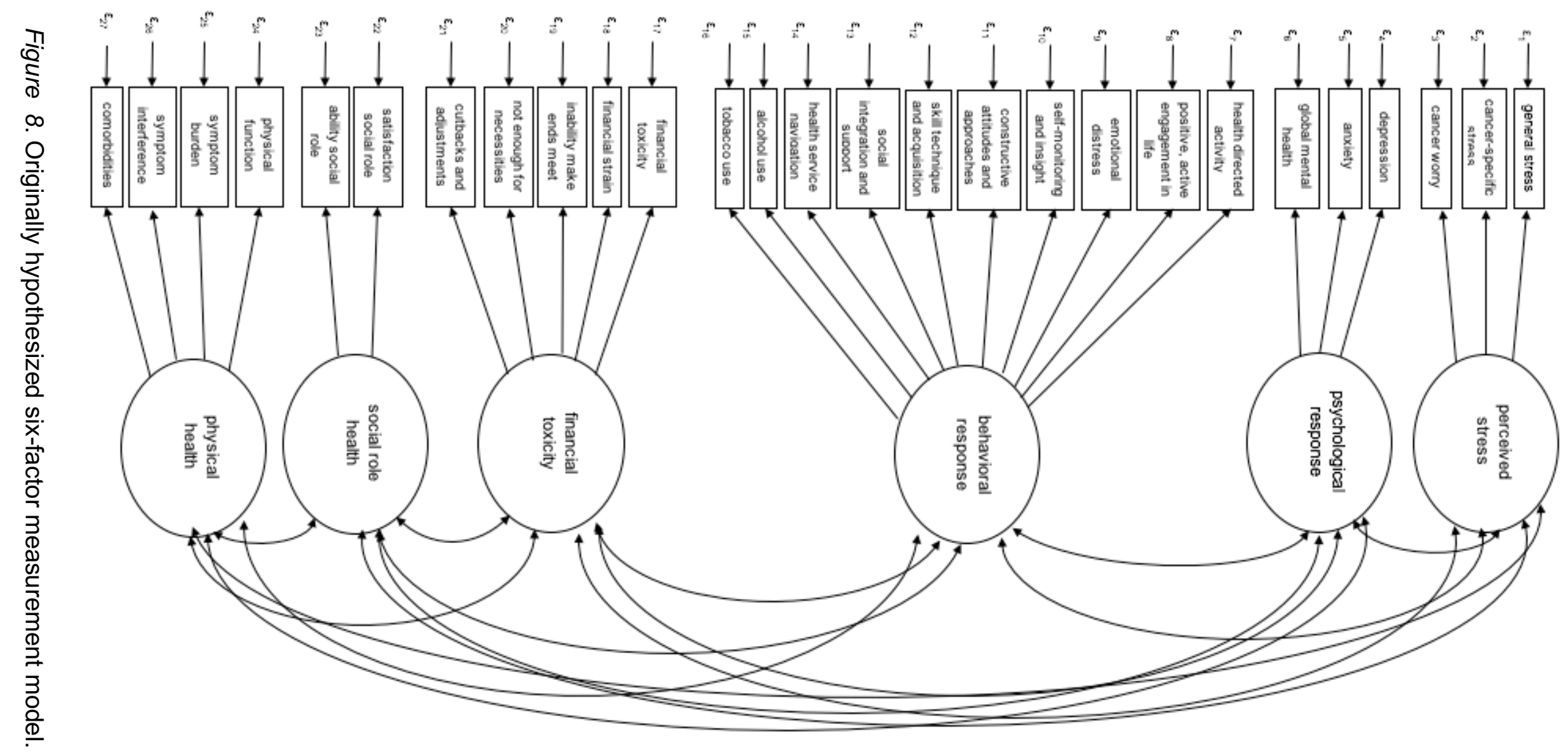


An examination of bivariate correlations and multicollinearity diagnostics suggested possible mild to moderate multicollinearity in the overall model, problematic model correlations, and a four-factor solution to the data. The following modifications, with specific rationales, were made as a result of the measurement model analyses. Measures of the stress and psychological response latent variables were reconceptualized and combined into a new latent variable, conceptualized as Distress, due to high correlations among these sets of variables. Measures of the social role function-ability and physical function latent variables were combined into a new latent variable, conceptualized as Functional Health, due to high correlations among these variables. The social role function satisfaction measure was dropped due to lack of conceptual congruence with functional health outcomes. The following observed behavioral response variables (the risky behaviors) were also dropped from the hypothesized measurement model: pack-year history and alcohol use (did not correlate well with other measures), heiQ emotional distress (cross-loaded onto many of the stress and behavioral response latent variables). Because of this, the behavioral response latent variable was renamed Self-Management Behaviors. Global mental health (cross-loaded onto the behavioral and psychological response latent variables) was also dropped from the measurement model. See modified four-factor measurement model in Figure 9. 


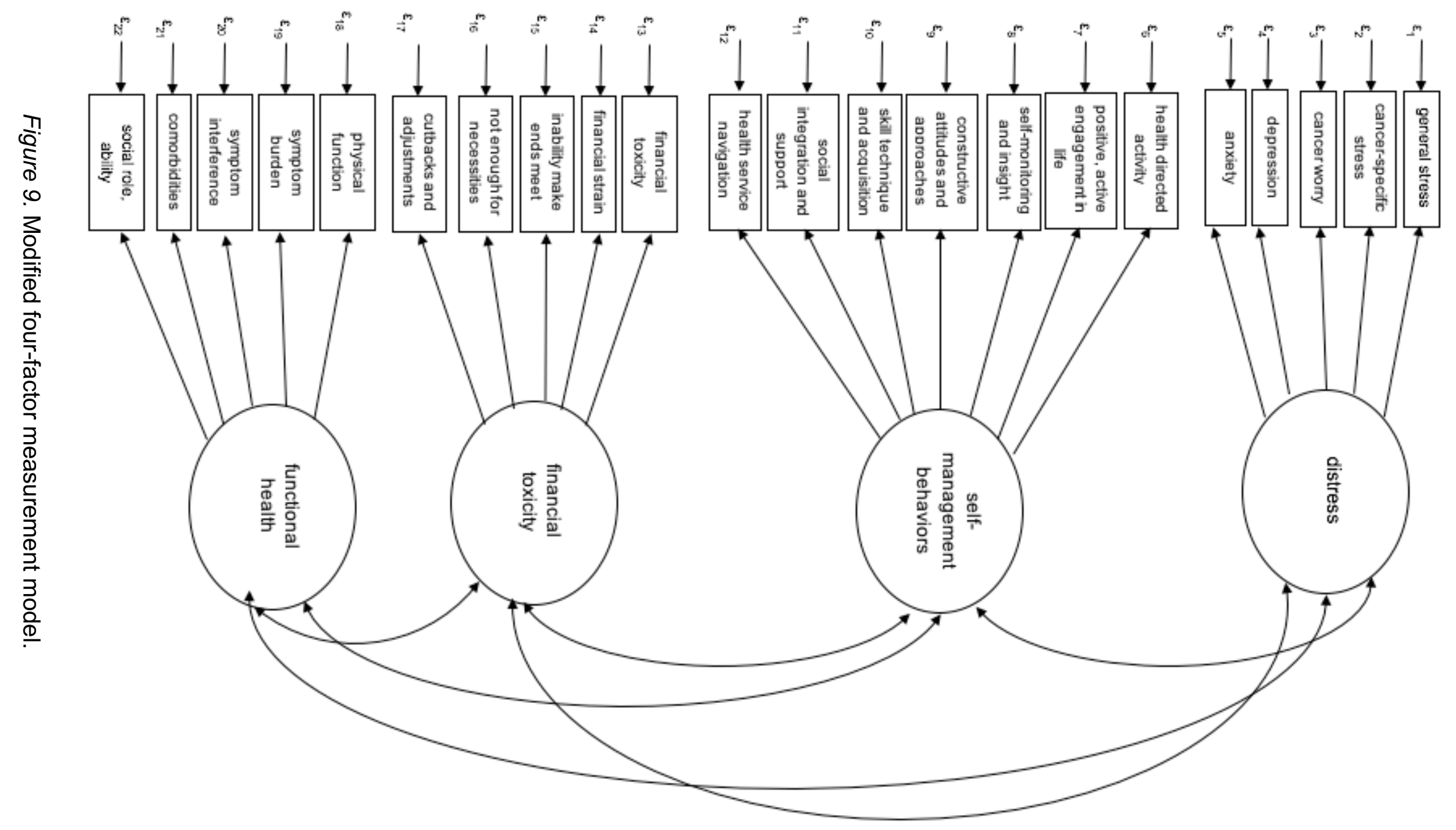


3.3.4.3.1 Model fit and parameter estimation. Support was found for the modified four-factor measurement model, $X^{2}(203, N=206)=455.57, p<.01 ; \mathrm{TLI}=.91, \mathrm{CFI}=.92, \mathrm{SRMR}=.06$, RMSEA $=.08,90 \%$ confidence interval $[\mathrm{CI}]$ for RMSEA $=[.07, .09]$.

To improve model fit, a series of post hoc model modifications were performed, allowing the following pairs of error terms to correlate: cancer-related stress and cancer worry; positive and active engagement in life and health directed activity; physical function and ability to participate in social roles and activities; social integration and support and self-monitoring and insight; and symptom distress and physical function. The final revised measurement model, illustrated in Figure 10, indicated a better fit $X^{2}(198, N=206)=331.32, p=.135 ; \mathrm{TLI}=.95, \mathrm{CFI}$ $=.96, \mathrm{SRMR}=.06, \mathrm{RMSEA}=.06,90 \% \mathrm{Cl}$ for $\mathrm{RMSEA}=[.05, .07]$. 


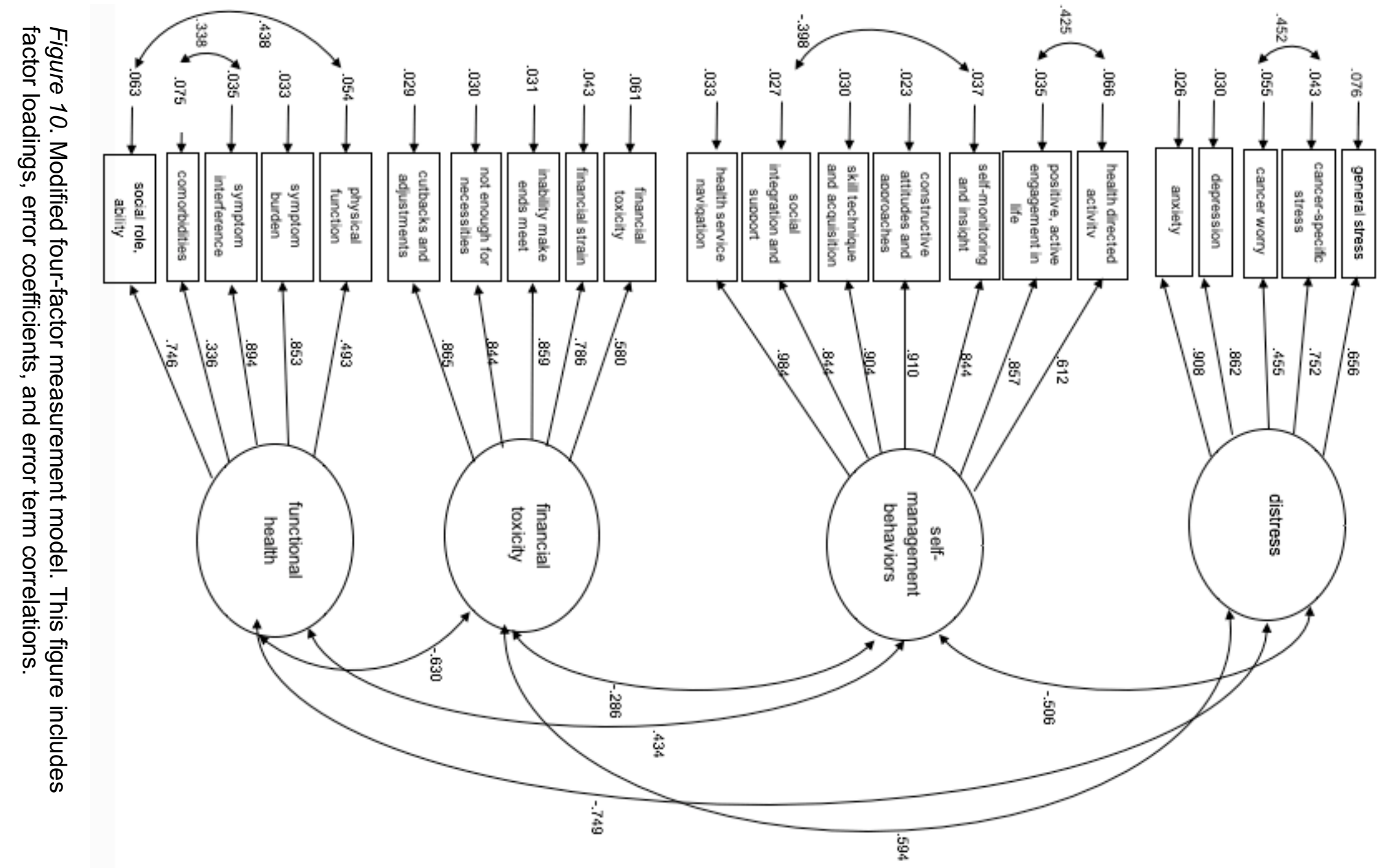




\subsubsection{Full structural equation model.}

3.3.4.4.1 The hypothesized model. The hypothesized full structural equation model based on the modified four-factor measurement model is presented in Figure 11, where, again, ovals represent latent variables, and rectangles represent measured variables. Absence of a line connecting variables implies no hypothesized direct effect. Curved lines indicate correlated error terms between measured variables.

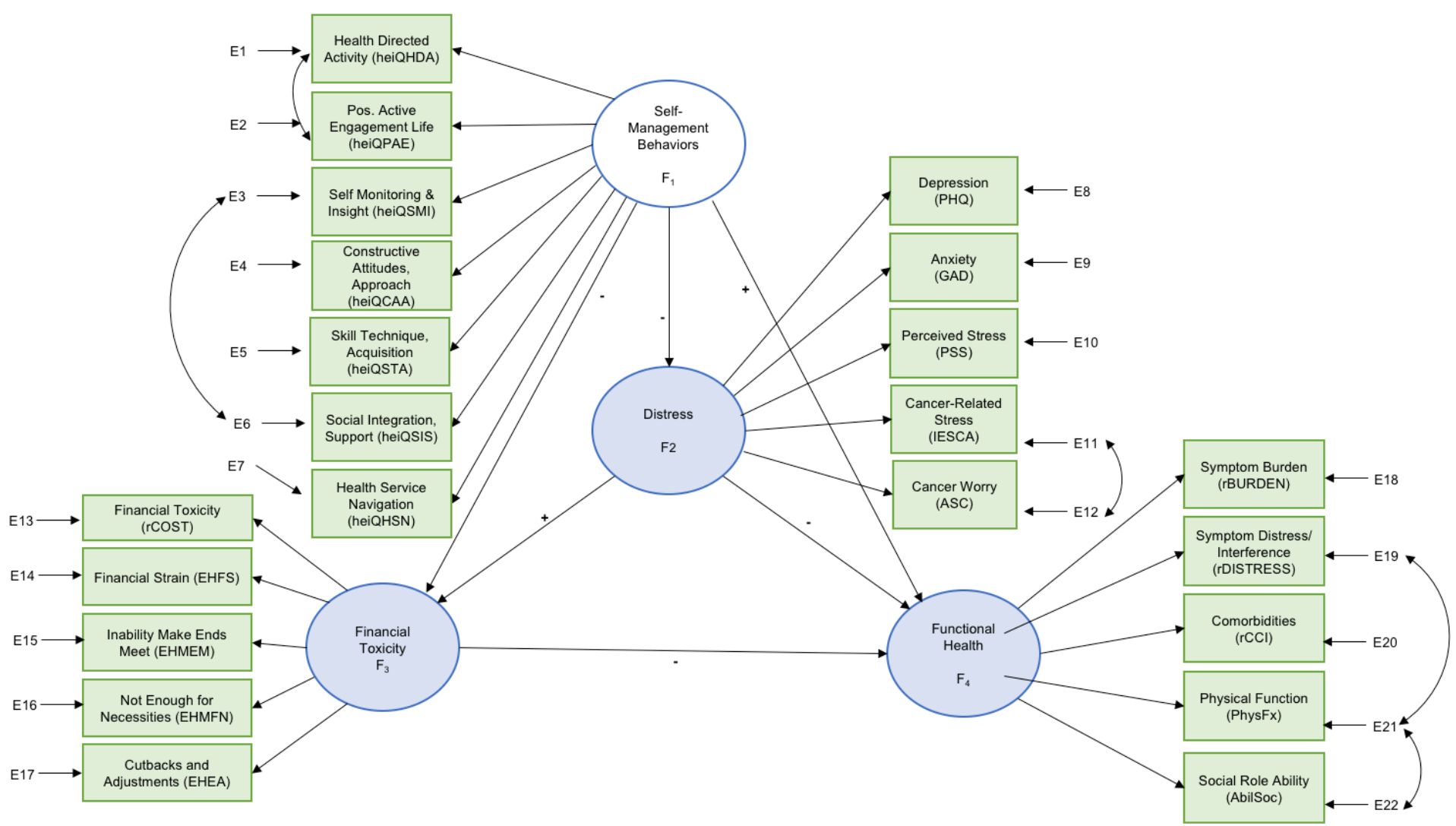

Figure 11. Hypothesized full structural equation model based on modified measurement model.

The hypothesized model examined predictors of financial toxicity and functional health. It was hypothesized that distress and self-management behaviors each directly predicted financial toxicity and functional health. Specifically, increased distress was hypothesized to directly increase financial distress and decrease functional health, and better self-management behaviors were hypothesized to directly decrease financial toxicity and increase functional 
health. It was also hypothesized that distress served as an intervening variable between selfmanagement behaviors and both financial toxicity and functional health.

Finally, it was hypothesized that, by adding upstream individual, sociodemographic, and clinical covariates, model fit would be improved. Specifically, the following variables were explored: personality (neuroticism and conscientiousness), age, social support, gender, educational attainment, marital status, difficulty paying for basic needs, years from first cancer diagnosis, years from treatment completion, time between first and second cancer diagnoses, and BMI.

3.3.4.4.2 Model fit and parameter estimation. Even though model fit was good based on study data $($ model 1$), X^{2}(198, N=206)=331.32, p<.01 ;$ TLI $=.95$, CFI $=.96$, SRMR $=.06$, RMSEA $=.06,90 \% \mathrm{CI}$ for RMSEA $=[.05, .07]$, there were two hypothesized paths that were not empirically supported: the direct path between self-management behaviors and financial toxicity (standardized coefficient $=0.02, p=.78$ ) and the direct path between self-management behaviors and functional health (standardized coefficient $=0.08, p=.32$ ).

Using model statistics and alignment with theoretical soundness, post hoc model modifications were performed in an attempt to develop a better fitting, more parsimonious model. Similar model fit was found by first removing the non-significant direct path between selfmanagement behaviors and financial toxicity (model 2), $X^{2}(199, N=206)=331.46, p<.010$; TLI $=.95, \mathrm{CFI}=.96, \mathrm{SRMR}=.06, \mathrm{RMSEA}=.06,90 \% \mathrm{Cl}$ for RMSEA $=[.05, .07]$, and then between self-management behaviors and functional health (model 3), $X^{2}(199, N=206)=331.93, p<.01$; $\mathrm{TLI}=.95, \mathrm{CFI}=.96, \mathrm{SRMR}=.06, \mathrm{RMSEA}=.06,90 \% \mathrm{Cl}$ for RMSEA $=[.05, .07]$. Lastly, modest improvement to model fit but increased parsimony was achieved by removing both direct paths between self-management behaviors and financial toxicity and functional health (model 4) $X^{2}$ $(200, N=206)=332.06, p<.01 ; \mathrm{TLI}=.95, \mathrm{CFI}=.96, \mathrm{SRMR}=.06, \mathrm{RMSEA}=.06,90 \% \mathrm{Cl}$ for RMSEA=[.05, .07], see Figure 12. 


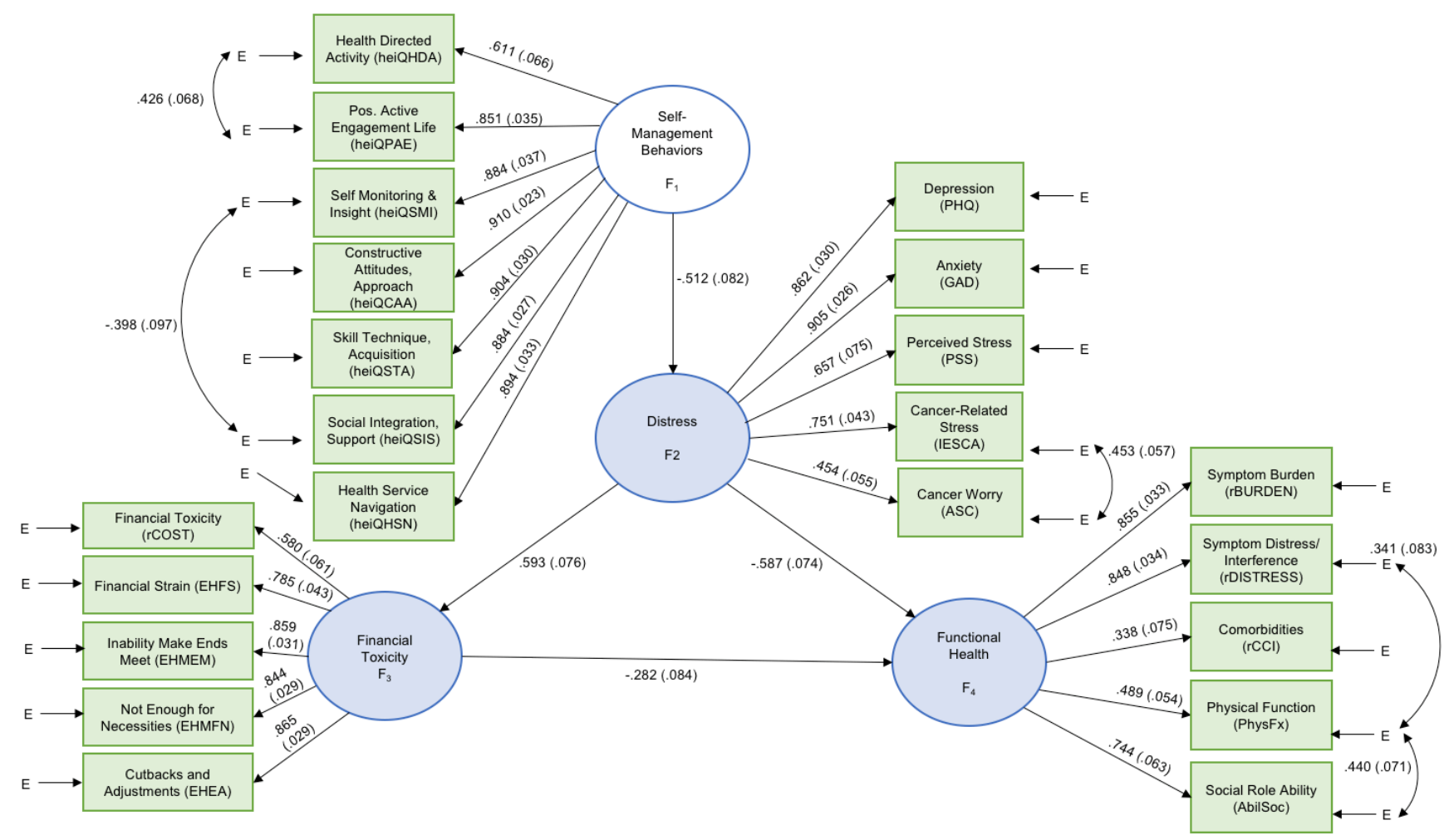

Figure 12. Final modified structural equation model based on modified measurement model. This figure includes standardized and unstandardized path coefficients and error term correlations.

3.3.4.5 Secondary aim. Additional exploratory modification testing was performed on model 4 to explore the impact of covariates as predictors of health behaviors on model fit. First, the model did not converge when all potential covariates were added as predictors of health behaviors. However, a final modified model that included neuroticism, social support, BMI (overweight versus normal and high risk [i.e., underweight plus obese]), and educational attainment (master's or higher versus less than high school and high school through bachelor's) predicting health behaviors and additional paths for neuroticism and social support predicting distress resulted in good model fit, $X^{2}(282, N=206)=511.13, p<.01 ;$ TLI $=.92$, CFI = .93, SRMR $=.06, \mathrm{RMSEA}=.06,90 \% \mathrm{Cl}$ for RMSEA $=[.05, .07]$. Figure 13 shows the final parsimonious model with modifications for the retained statistically significant covariates. 


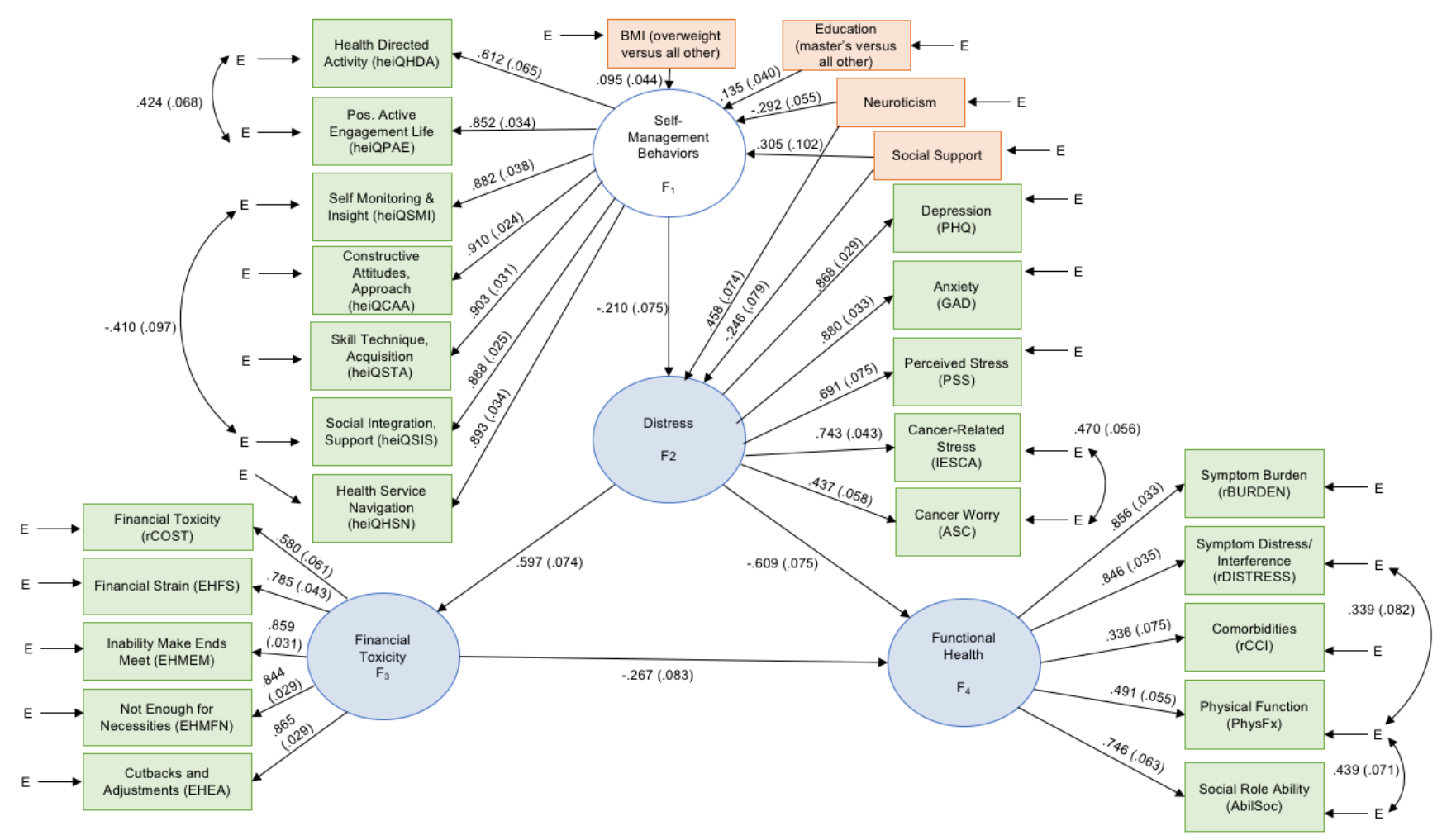

Figure 13. Final parsimonious structural equation model based on modified measurement model with significant covariates retained. This figure includes standardized and unstandardized path coefficients and error term correlations.

3.3.4.5.1 Direct effects. As hypothesized, increased financial toxicity was predicted by greater distress (standardized path coefficient $=.60, \mathrm{p}<.01)$ and poorer functional health was predicted by greater distress (standardized path coefficient $=-.61, p<.01$ ) and by financial toxicity (standardized path coefficient $=-.23, p<.01)$. Increased distress was predicted by poorer selfmanagement behaviors, greater neuroticism, and lower perceived social support (standardized path coefficients $=-.21, .46$, and -.25 , respectively, $\mathrm{p}<.01$ ). BMI (overweight), education (master's or greater), decreased neuroticism, and better perceived social support predicted better health behaviors (standardized path coefficients $=.10, .14,-.29, .31$, respectively, $p<.01$ ) Better behavioral responses did not significantly predict financial toxicity or functional health (unstandardized path coefficients $=.12$ and .04 , respectively, $p \geq .05$ ). 


\subsubsection{Discussion}

Using an adapted psychobehavioral stress-response model, this study was the first to identify pathways and individual risk factors associated with health outcomes in adults with MPC. The data in this study fit a modified four-factor measurement model, with latent variables including self-management, distress (combined perceived stress and psychological distress), financial toxicity, and functional health (combined social health and physical health). Self-management was the upstream latent variable identified in the final modified model; self-management behavior predicted the other latent variables, either directly (i.e., predicting distress) or indirectly (i.e., predicting financial toxicity and functional health through distress). In the expanded model, which included possible predictors (i.e., potential risk factors of poor self-management and increased distress), overweight BMI, graduate education, less neuroticism, and increased social support predicted better self-management; poorer self-management, greater neuroticism, and lower social support predicted increased distress.

Our originally hypothesized six-factor model was not empirically supported by the data in this study. As a result, latent variables, associated measurement variables, and pathway configurations were modified and reconceptualized based on empirical support and congruence with the literature, yielding a four-factor model. The four-factor model, with combined perceived stress/psychological response ("distress") and social role function/physical health ("functional health") variables, highlights important pathways among self-management behaviors, distress, financial toxicity, and functional health. Future studies should evaluate the validity of combining these measures. It is possible that the high correlations among these sets of variables was a consequence of study design and/or measurement issues. The original conceptualization of the perceived stress latent variable, the perception that one's demands overwhelm one's coping resources (Lazarus \& Folkman, 1984), was meant to capture current stressors of general life demands, cancer-specific stress, and cancer worry. Conversely, the psychological response latent variable was mean to capture the longer-term (maladaptive) responses to the chronic 
stress of cancer (National Comprehensive Cancer Network, 2018a) and included measures of depressive symptoms, anxiety, and mental health. It is possible that the cross-sectional nature of this study precluded distinguishing between these short- and long-term responses in MPC survivors who had been living with their diagnoses for, in some cases, multiple years. Similarly, social role ability and physical health measures loaded onto a combined factor, which we conceptualized as functional health. Ability to participate in social roles and activities does reflect a functional ability, but, again, further research is necessary to evaluate the validity of combining these different measures into a single latent variable. Finally, the PROMIS Global Mental Health measure we attempted to incorporate into this model loaded across many of the latent variables in the model, preventing us from using it in multivariate analyses.

Importantly, the four key constructs (self-management, distress, financial toxicity, and functional health outcomes) and their inter-relationships demonstrated in the final model, provide clear direction for future studies. Distress, which was predicted by self-management behaviors, significantly predicted both increased financial toxicity and poorer functional health. We hypothesize that increased distress may be impairing an individual's productivity, leading to financial toxicity, and that financial toxicity may be impacting an individual's functional health (e.g., through medication adherence). Further, we hypothesize that distress may be affecting functional health through biological pathways (e.g., triggered glucocorticoid receptor resistance, immune dysregulation, and risk for disease) (Cohen et al., 2012). It is also possible that mechanisms impacting an individual's risk for MPC (e.g., genetic predisposition, previous cancer treatment, negative health behaviors, etc.) may also influence health outcomes in this survivor population. Future studies should identify the individual and interacting influence of these different potential mechanisms.

Not all of the originally hypothesized pathways were found to be statistically significant. The direct pathway between self-management behaviors and financial toxicity was neither significant nor was it in the direction we had hypothesized; for financial toxicity, distress was a 
more important direct predictor, while distress mediated the relationship between selfmanagement and financial toxicity. Also, while the path coefficient did reflect the hypothesized direction, self-management behaviors also were not a significant direct predictor of functional health. The relationship, again, was mediated by distress.

Limitations are acknowledged in this study. Despite adequate power to conduct analyses, the response rate to study mailings (15.2\%) was lower than expected and could have introduced bias into study findings. Several findings mitigate this concern. Study participants were similar in age, gender, ethnicity, and marital status to participants in previously recruited national MPC samples, despite being recruited from a regional cancer registry. Perhaps more importantly, study participants did not differ from nonparticipants identified by the Cancer Registry as potentially eligible for participation in this study on key variables including age, sex, race, marital status, primary payer at first diagnosis, years since first cancer diagnosis, and first cancer site. Also, while path analysis can seem to imply temporal or even causal relationships, the cross-sectional design of this study limits interpretation to associations among variables. Longitudinal studies could help to identify the temporal nature of relationships, while experimental studies will be required to understand whether relationships in the proposed model are causal in nature.

Several unique aspects of this sample and contributions to the science are noteworthy. First, as a result of our inclusion criteria, record validated clinical data verified that participants in this sample were closer to their first diagnosis $(M=5.8$ years, $S D=2.9)$, as compared to $11-17$ years in other studies, providing the opportunity to examine a new cohort of MPC survivors not previously described (Belcher et al., 2016; Burris \& Andrykowski, 2011; Gotay et al., 2007; Thong et al., 2013). This study also included cancer sites not frequently represented in MPC literature (i.e., lung and thyroid cancer). Additionally, this sample had higher than average BMI and rates of obesity $(40.7 \%)$ when compared to the general U.S. population $(36.5 \%)$, cancer survivors (31.1\%) and other published MPC literature (27.4\%) (Burris \& Andrykowski, 2011; 
Centers for Disease Control and Prevention, 2017; National Cancer Institute, 2018b). While not successful broadening racial and ethnic diversity of the literature in this study, we did recruit a sample of participants with a broad range of educational attainment, which is representative of the broader U.S. population (Ryan \& Bauman, 2016). Importantly, educational attainment was identified as an important predictor of self-management in Aim 2 covariate analyses.

The data in this study support healthy self-management behaviors as vitally important to positive health outcomes in MPC survivors, and MPC survivors should be evaluated for poor health behaviors and distress, both of which are modifiable. This is particularly important among the subset of MPC patients at increased risk for poor health outcomes (i.e. those with less education, less social support, overweight BMI, and greater neuroticism). The National Cancer Institute recommends that individuals with MPC diagnoses be considered candidates for cancer risk assessment and counseling (i.e., clinical assessment, applicable genetic testing, and recommendations of risk management counseling) to guide screening and risk reduction interventions (National Cancer Institute, 2018a). Additionally, survivorship clinical practice guidelines (National Comprehensive Cancer Network, 2018b, 2018a) recommend clinical assessment of and individualized recommendations for healthy lifestyle and provide direction on ways to assess for and address key topics such as diet, activity, caloric balance, and brief distress screenings. Based on these data, clinicians should also increase their awareness of financial toxicity and engage their patients in care value discussions. As evidenced by the expertise needed to address the identified key clinical priorities in MPC patients, collaboration and communication among specialists (e.g., nutritionists, behavioral change experts, mental health professionals, financial experts, primary care providers, oncologists [potentially including more than one medical or surgical oncology team for cancer disease site specialists], and genetic counselors) is key.

These findings are also relevant to policy discussions. As recognized by recent literature documenting increasing rates of MPC in cancer survivors (Davidson, 2017; Murphy et al., 
2017), clinical trial eligibility criteria should be evaluated to determine representativeness of the U.S. cancer survivor population. Whenever possible, MPC status should not be an explicit exclusion criterion for clinical trial participation. Also, organizations should strive to create systems and policies that allow for continuity of care among providers in medically complex patients.

Future studies should evaluate and expand upon this refined model for studying health outcomes in adults with MPC, with a particular focus on self-management and financial toxicity, two newly highlighted areas of importance, and on identification of the individual and interacting influences of potential mechanisms impacting health outcomes in MPC survivors. Biological pathways also are an untapped line of inquiry in this patient population and could lead to increased understanding of the mechanisms linking distress to poor outcomes in this model. It is also important to move toward understanding how outcomes may differ among different subsets of MPC survivors (e.g., childhood versus adulthood diagnoses; MPCs associated with genetic cancer syndromes versus treatment-related MPCs versus MPCs associated with risky behaviors), as risk factors and health outcomes may vary among groups. It is possible that mechanisms impacting an individual's risk for MPC (e.g., genetic predisposition, previous cancer treatment, negative health behaviors, etc.) may also influence health outcomes in this survivor population and warrants future analysis.

Study design and recruitment are important considerations for the advancement of MPC science. Future research should move to longitudinal studies to 1) establish temporal relationships among key variables; 2) understand how MPC survivorship risk factors change over time; and 3) identify vulnerable phases in the MPC survivorship trajectory. With increased demands being placed on people's time and attention, survey response rates have been declining and costs have been rising (National Science Foundation, n.d.), and this type of data collection may be becoming outdated when attempting to recruit generalizable samples. Future MPC studies should also focus on novel recruitment methods of this hard to reach patient 
population, as survivors do not all attend specialty MPIC clinics where targeted recruitment could occur. Attention should also be paid to addressing reasons provided for nonparticipation in this study (i.e., increased age, perceived ineligibility, privacy/accessing medical records). Future research must also focus on conducting studies that are sensitive to the challenges and perspectives of more diverse groups of MPC survivors.

\subsubsection{Funding}

This research was supported by a Doctoral Degree Scholarship in Cancer Nursing (DSCNR-17077) from the American Cancer Society, the Robert Wood Johnson Foundation Future of Nursing Scholars program, the Nightingale Awards of Pennsylvania PhD Scholarship, and the University of Pittsburgh School of Nursing Margaret E. Wilkes Scholarship (Belcher).

\subsubsection{Conflict of interest disclosures}

The authors have no conflicts of interest to disclose.

\subsubsection{Acknowledgements}

The authors wish to acknowledge the individuals who participated in this study; Sharon Winters and Althea Schneider for cancer registry expertise; Emilie Hausmann, Olivia D'Antonio, and Kathryn Corey for administrative support; and the physician experts who championed the study, including: Leonard Appleman, Adam Brufsky, Edward Chu, Robert Edwards, Jeffrey Gingrich, John Kirkwood, Jonas Johnson, Arjun Pennathur, Liza Villaruz, and Linwah Yip. 


\subsubsection{Supplementary online material}

\begin{tabular}{lc} 
Table 9 \\
$\begin{array}{l}\text { Frequencies of Second Cancer Diagnosis Sites } \\
\text { (N=205) }\end{array}$ \\
\hline Site & $\underline{\mathrm{n}(\%)}$ \\
Breast & $38(18.5)$ \\
Lung / Bronchus & $29(14.1)$ \\
Thyroid & $19(9.3)$ \\
Kidney / Renal Pelvis & $18(8.8)$ \\
Melanoma & $16(7.8)$ \\
Prostate & $15(7.3)$ \\
Lymphoma & $14(6.8)$ \\
Uterine Corpus (Endometrial) & $13(6.3)$ \\
Colorectal & $9(4.4)$ \\
Urinary Bladder & $9(4.4)$ \\
Oral Cavity, Pharynx, Larynx & $7(3.4)$ \\
Ovarian & $5(2.4)$ \\
Leukemia & $2(1.0)$ \\
Multiple Myeloma & $2(1.0)$ \\
Neuroendocrine Tumor & $2(1.0)$ \\
Cervical & $1(0.5)$ \\
Liver & $1(0.5)$ \\
Merkel Cell & $1(0.5)$ \\
Pancreatic & $1(0.5)$ \\
Sarcoma & $1(0.5)$ \\
Vulvar & $1(0.5)$ \\
Unknown Origin & $1(0.5)$ \\
\hline Note. Data were not able & \\
\hline
\end{tabular}

Note. Data were not able to be obtained from medical records for 6 cases. 
Table 10

Frequencies of Sample First or Second Cancer

Diagnoses Sites $(N=211)$

$\begin{array}{lc}\text { Site } & \frac{\mathrm{n}(\%)}{7(22.3)} \\ \text { Breast } & 45(21.3) \\ \text { Prostate } & 42(19.9) \\ \text { Lung / Bronchus } & 31(14.7) \\ \text { Uterine Corpus (Endometrial) } & 29(13.7) \\ \text { Thyroid } & 28(13.3) \\ \text { Kidney / Renal Pelvis } & 26(12.3) \\ \text { Melanoma } & 23(10.9) \\ \text { Colorectal } & 20(9.5) \\ \text { Urinary Bladder } & 14(6.6) \\ \text { Lymphoma } & 13(6.2) \\ \text { Ovarian } & 11(5.2) \\ \text { Oral Cavity, Pharynx, Larynx } & 2(0.9) \\ \text { Leukemia } & 2(0.9) \\ \text { Multiple Myeloma } & 2(0.9) \\ \text { Neuroendocrine Tumor } & \end{array}$

Note. Data were not able to be obtained from medical records for 6 second cancer cases.

Additional single cases included cervical, liver, merkel cell, pancreatic, sarcoma, vulvar, and unknown primary sites. 
Table 11

Sample Patterns of First and Second Cancer Diagnoses Sites $(N=205)$

\begin{tabular}{|c|c|c|}
\hline First Cancer Site & Second Cancer Site & $\underline{\mathrm{n}(\%)}$ \\
\hline$\overline{\text { Lung }}$ & Lung & $20(9.8)$ \\
\hline Breast & Breast & $17(8.3)$ \\
\hline Thyroid & Thyroid & $12(5.9)$ \\
\hline Melanoma & Melanoma & $11(5.4)$ \\
\hline Bladder & Prostate & $10(4.9)$ \\
\hline Renal & Renal & $7(3.4)$ \\
\hline Prostate & Bladder & $6(2.9)$ \\
\hline Prostate & Lymphoma & $6(2.9)$ \\
\hline Uterine & Breast & $6(2.9)$ \\
\hline Thyroid & Breast & $4(2.0)$ \\
\hline Prostate & Colorectal & $4(2.0)$ \\
\hline Prostate & Renal & $4(2.0)$ \\
\hline Colorectal & Breast & $4(2.0)$ \\
\hline Breast & Uterine & $3(1.5)$ \\
\hline Ovarian & Uterine & $3(1.5)$ \\
\hline Prostate & Lung & $3(1.5)$ \\
\hline Uterine & Ovarian & $3(1.5)$ \\
\hline Renal & Prostate & $3(1.5)$ \\
\hline Oral Cavity/Pharynx & Oral Cavity/Pharynx & $3(1.5)$ \\
\hline Breast & Renal & $2(1.0)$ \\
\hline Thyroid & Uterine & $2(1.0)$ \\
\hline Ovarian & Breast & $2(1.0)$ \\
\hline Prostate & Melanoma & $2(1.0)$ \\
\hline Prostate & Oral Cavity/Pharynx & $2(1.0)$ \\
\hline Colorectal & Uterine & $2(1.0)$ \\
\hline Colorectal & Lung & $2(1.0)$ \\
\hline Uterine & Uterine & $2(1.0)$ \\
\hline Uterine & Lymphoma & $2(1.0)$ \\
\hline Melanoma & Breast & $2(1.0)$ \\
\hline Melanoma & Thyroid & $2(1.0)$ \\
\hline Renal & Lymphoma & $2(1.0)$ \\
\hline Lung & Breast & $2(1.0)$ \\
\hline Lung & Renal & $2(1.0)$ \\
\hline Breast & Thyroid & $1(0.5)$ \\
\hline Breast & Ovarian & $1(0.5)$ \\
\hline Breast & Leukemia & $1(0.5)$ \\
\hline Thyroid & Prostate & $1(0.5)$ \\
\hline Thyroid & Colorectal & $1(0.5)$ \\
\hline Thyroid & Melanoma & $1(0.5)$ \\
\hline Thyroid & Lung & $1(0.5)$ \\
\hline Thyroid & Leukemia & $1(0.5)$ \\
\hline Ovarian & Lymphoma & $1(0.5)$ \\
\hline Ovarian & Pancreatic & $1(0.5)$ \\
\hline Prostate & Multiple Myeloma & $1(0.5)$ \\
\hline Prostate & Neuroendocrine Tumor & $1(0.5)$ \\
\hline Colorectal & Colorectal & $1(0.5)$ \\
\hline
\end{tabular}


Table 11 (continued).

\begin{tabular}{|c|c|c|}
\hline Colorectal & Bladder & $1(0.5)$ \\
\hline Colorectal & Renal & $1(0.5)$ \\
\hline Colorectal & Laryngeal & $1(0.5)$ \\
\hline Colorectal & Lymphoma & $1(0.5)$ \\
\hline Colorectal & Liver & $1(0.5)$ \\
\hline Bladder & Bladder & $1(0.5)$ \\
\hline Bladder & Renal & $1(0.5)$ \\
\hline Uterine & Colorectal & $1(0.5)$ \\
\hline Uterine & Melanoma & $1(0.5)$ \\
\hline Uterine & Renal & $1(0.5)$ \\
\hline Uterine & Thyroid & $1(0.5)$ \\
\hline Uterine & Cervical & $1(0.5)$ \\
\hline Uterine & Neuroendocrine Tumor & $1(0.5)$ \\
\hline Uterine & Vulvar & $1(0.5)$ \\
\hline Melanoma & Prostate & $1(0.5)$ \\
\hline Melanoma & Bladder & $1(0.5)$ \\
\hline Melanoma & Lung & $1(0.5)$ \\
\hline Melanoma & Lymphoma & $1(0.5)$ \\
\hline Melanoma & Merkel Cell & $1(0.5)$ \\
\hline Renal & Breast & $1(0.5)$ \\
\hline Renal & Lung & $1(0.5)$ \\
\hline Renal & Thyroid & $1(0.5)$ \\
\hline Renal & Ovarian & $1(0.5)$ \\
\hline Oral Cavity/Pharynx & Colorectal & $1(0.5)$ \\
\hline Oral Cavity/Pharynx & Lung & $1(0.5)$ \\
\hline Oral Cavity/Pharynx & Thyroid & $1(0.5)$ \\
\hline Oral Cavity/Pharynx & Multiple Myeloma & $1(0.5)$ \\
\hline Lung & Colorectal & $1(0.5)$ \\
\hline Lung & Uterine & $1(0.5)$ \\
\hline Lung & Melanoma & $1(0.5)$ \\
\hline Lung & Oral Cavity/Pharynx & $1(0.5)$ \\
\hline Lung & Thyroid & $1(0.5)$ \\
\hline Lung & Lymphoma & $1(0.5)$ \\
\hline Lung & Sarcoma & $1(0.5)$ \\
\hline Lung & Unknown Origin & $1(0.5)$ \\
\hline
\end{tabular}

Note. Second cancer data were not able to be obtained from medical records for 6 cases. 
Table 12

Most Common Second Cancer Sites within Each First Primary

Cancer Site Group ( $N=205)$

\begin{tabular}{|c|c|c|}
\hline First Cancer Site & Second Cancer Site & $\underline{\mathrm{n}(\%)}$ \\
\hline Lung/Bronchus* $(n=32)$ & Lung/Bronchus & $20(62.5)$ \\
\hline \multirow[t]{2}{*}{ Prostate $^{*}(n=29)$} & Bladder & $6(20.7)$ \\
\hline & Lymphoma & $6(20.7)$ \\
\hline reast $^{*}(n=25)$ & Breast & $17(69.0)$ \\
\hline hyroid & Thyroid & $12(54.5)$ \\
\hline * $(n=20)$ & Melanoma & $11(55.0)$ \\
\hline rpus (n=20) & Breast & $6(30.0)$ \\
\hline al Pelvis* $(n=16$ & Kidney/Renal Pelvis & $7(43.8)$ \\
\hline$(n=14)$ & Breast & $4(28.6)$ \\
\hline dder $(n=12)$ & Prostate & $10(83.3)$ \\
\hline Ovarian $(n=8)$ & Uterine & $3(37.5)$ \\
\hline Oral Cavity/Pharynx $(n=7)$ & Oral Cavity/Pharynx & $3(42.9)$ \\
\hline
\end{tabular}

Note. Data were not able to be obtained from medical records for 6 cases ( ${ }^{*}$ one case per each disease site indicated).

Percentages were calculated based on number of total cases per first cancer site. 
Table 13

Sociodemographic and Clinical Comparisons of Cancer Registry Sociodemographic and Clinical Data:

Participants versus Nonparticipants

\begin{tabular}{|c|c|c|c|c|c|c|}
\hline \multirow{4}{*}{$\begin{array}{l}\text { Age Mean }(S D) \\
\text { Years Since First Cancer } \\
\text { Diagnosis Mean (SD) }\end{array}$} & \multicolumn{2}{|c|}{$\begin{array}{l}\text { Participants } \\
n=215\end{array}$} & \multicolumn{2}{|c|}{$\begin{array}{c}\text { Nonparticipants } \\
n=1176\end{array}$} & \multirow{2}{*}{$\begin{array}{l}\text { Test Statistic* } \\
\stackrel{(d f)}{t(1389)=0.502}\end{array}$} & \multirow{2}{*}{$\frac{\mathrm{p} \text {-value }}{.248}$} \\
\hline & 67.2 & 11.5 & 67.7 & 12.1 & & \\
\hline & 5.8 & 2.8 & 5.6 & 2.9 & $t(1384)=-0.916$ & .176 \\
\hline & $\underline{n}$ & $\underline{\%}$ & $\underline{n}$ & $\underline{\%}$ & & \\
\hline \multicolumn{7}{|l|}{ Sex } \\
\hline Male & 85 & 39.5 & 512 & 43.5 & $X^{2}(1)=1.189$ & .276 \\
\hline Female & 130 & 60.5 & 664 & 56.5 & & \\
\hline Race & & & & & $x^{2}(1)=2.323$ & .136 \\
\hline White & 200 & 93.0 & 1049 & 89.7 & & \\
\hline Other & 15 & 7.0 & 121 & 10.3 & & \\
\hline Marital Status & & & & & $X^{2}(1)=2.358$ & .125 \\
\hline Married/Living with a Partner & 147 & 72.1 & 739 & 66.6 & & \\
\hline Other & 57 & 27.9 & 371 & 33.4 & & \\
\hline $\begin{array}{l}\text { Primary Payer at First Cancer } \\
\text { Diagnosis }\end{array}$ & & & & & $x^{2}(2)=4.812$ & .090 \\
\hline Private Insurance & 129 & 66.5 & 637 & 58.5 & & \\
\hline Medicare, Tricare, or VA & 53 & 27.3 & 384 & 35.3 & & \\
\hline Medicaid & 12 & 6.2 & 67 & 6.2 & & \\
\hline First Cancer Site & & & & & $x^{2}(2)=13.319$ & .206 \\
\hline Breast & 26 & 12.6 & 180 & 15.3 & & \\
\hline Prostate & 32 & 14.9 & 117 & 9.9 & & \\
\hline Colorectal & 15 & 7.0 & 121 & 10.3 & & \\
\hline Bladder & 12 & 5.6 & 86 & 7.3 & & \\
\hline Uterine & 20 & 9.3 & 109 & 9.3 & & \\
\hline Melanoma & 21 & 9.8 & 97 & 8.2 & & \\
\hline Kidney/Renal Pelvis & 17 & 7.9 & 116 & 9.9 & & \\
\hline Oral Cavity/Pharynx & 7 & 3.3 & 65 & 5.5 & & \\
\hline Lung/Bronchus & 33 & 15.3 & 139 & 11.8 & & \\
\hline Thyroid & 23 & 10.7 & 107 & 9.1 & & \\
\hline Ovarian & 8 & 3.7 & 39 & 3.3 & & \\
\hline
\end{tabular}

Notes. SD = standard deviation

${ }^{*}$ Reported statistics are Pearson chi-square test of independence and independent samples t-tests. 


\subsection{DESCRIPTION OF SELF-MANAGEMENT BEHAVIORS (DISSERTATION AIM 3)}

\subsection{THE IMPORTANCE OF SELF-MANAGEMENT BEHAVIORS}

Previous literature has demonstrated poorer health in MPC survivors versus their single cancer counterparts. Cancer diagnosis and the post-treatment survivorship phase can result in both negative and positive influence on health behaviors (Carmack, Basen-Engquist, \& Gritz, 2011; Park, Edmondson, Fenster, \& Blank, 2008). However, unhealthier behaviors have been documented in MPC survivors (Burris \& Andrykowski, 2011), including greater likelihood of physical inactivity, greater alcohol consumption when drinking (though less likely to use alcohol overall), and greater likelihood of cigarette and smokeless tobacco use. Given known health deficits in this population of cancer survivors, positive self-management behaviors represent targets (Risendal et al., 2015), providing opportunities to interrupt negative downstream health outcomes and maintain and/or restore optimal wellness.

Corbin and Strauss first identified the process of self-management by describing the work (e.g., medical management, behavioral management, and emotional management) of living with a chronic illness (Corbin \& Strauss, 1988). As this day-to-day work of managing one's health is shifting from providers to survivors (Barlow et al., 2002) and their family members, studies in patients with chronic illness (Grady \& Gough, 2014; Grey et al., 2015; Lorig \& Holman, 2003) and general cancer (Chen et al., 2015; Hammer et al., 2015; McCorkle et al., 2011; Miller et al., 2009; Risendal et al., 2015) have established that self-management is both a public health and clinical priority. The National Institutes of Health and others (Grady, Daley, \& Gough, 2014; Grady \& Gough, 2014; Knobf et al., 2015; National Cancer Survivorship Resource Center, n.d.; National Institute of Health, 2017; Rudy \& Grady, 2005) have called for biobehavioral research to identify mechanisms underlying self-management behaviors.

No prior work has examined how MPC survivors self-manage their health. It is vital to identify the individual characteristics (i.e., personal, clinical, sociodemographic) that are 
associated with self-management in MPC survivors and to understand mechanisms linking selfmanagement to health outcomes to 1) better characterize at risk MPC survivors and 2) better understand mechanisms linking self-management to health outcomes. Targeted and tailored self-management interventions, which have been shown to provide positive benefit in varying ethnic, geographic, and age groups in other patient populations (Grady \& Gough, 2014), could reduce long-term health problems experienced by MPC survivors.

In Aim 3 of this dissertation study, we describe potentially modifiable health behaviors by analyzing the heiQ items, alcohol use, tobacco use, and BMI measures in detail.

\subsection{RESULTS}

\subsubsection{Self-management domain scores}

Table 14 displays scores for self-management domains, as measured by the heiQ (no score alterations applied). In general, most mean heiQ subscale scores were above 3.0, though ranges demonstrate good variability in the scale scores. Health Directed Behavior, which includes items that reflect incorporation of healthy behaviors (e.g., physical and healthy activity) into one's lifestyle, had the lowest subscale score (Mean=2.9, SD=0.7) and lowest observed minimum scores (1.0).

Table 14

Raw Scores for Self-Management Domains, as Measured by Health Education Impact Questionnaire Measure Subscales $(N=208)$

\begin{tabular}{|c|c|c|}
\hline \multirow[b]{2}{*}{ Domains } & \multicolumn{2}{|c|}{ Result } \\
\hline & Mean (SD) & Range \\
\hline Health Directed Activity & $2.9(0.7)$ & $\overline{1.0-4.0}$ \\
\hline Positive and Active Engagement in Life & $3.3(0.6)$ & $1.6-4.0$ \\
\hline Emotional Distress* & $3.1(0.6)$ & $1.3-4.0$ \\
\hline Self-Monitoring and Insight & $3.3(0.4)$ & $2.0-4.0$ \\
\hline Constructive Attitudes and Approaches & $3.4(0.5)$ & $1.4-4.0$ \\
\hline Skill and Technique Acquisition & $3.2(0.5)$ & $1.5-4.0$ \\
\hline Social Integration and Support & $3.2(0.6)$ & $1.4-4.0$ \\
\hline Health Service Navigation & $3.4(0.5)$ & $1.8-4.0$ \\
\hline
\end{tabular}

Notes. SD=standard deviation.

${ }^{*}$ Emotional distress is interpreted inversely, with higher scores indicating increased negative impact of health on emotions. 


\subsubsection{Self-management behavior item scores}

Frequencies and percentages of self-management behaviors by individual items are presented in Table 15 . Across items, only about $54-78 \%$ of individuals agreed that they were engaging in regular health directed activities, with the lowest percentage observed being for the walking for exercise item (53.8\%). Approximately $82-95 \%$ of individuals agreed that they participated in activities to positively and actively engage in life. Approximately $13-48 \%$ of individuals agreed with items in the emotional distress domain, with nearly $50 \%$ of participants agreeing that they often worried about their health. Approximately $80-98 \%$ agreed with doing things reflective of self-monitoring and insight; the lowest frequency in this domain was knowing what triggers and makes health problems worse (79.8\%). Frequency of agreement with items in the constructive attitudes and approaches and skill technique acquisition ranged from high 80's-90's. Social integration and support varied by item (78.4-92.8\%); 78.4\% of participants felt that family and caregivers understood what they were going through when feeling ill. Lastly, frequencies for health service navigation were approximately $90 \%$ or above.

Table 15

Frequencies of participants who agree with performing self-management behaviors, by domain and item $(N=208)$ Item Results

$\underline{\text { Individual Items by Domain }}$

Health Directed Activity

Activity at least 1 day per week to improve health

At least 1 type of physical activity daily for 30 minutes

Set aside time for healthy activities on most days of week

Walk for exercise at least 15 minutes per day most days of week

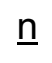

161

154

149

112

Positive and Active Engagement in Life

Do some of things I really enjoy on most days

Try to make most of life

Doing interesting things in my life

Have plans to do enjoyable things for self during next few days

Feel like actively involved in life

Emotional Distress*

Often worry about health

Health problems make me dissatisfied with life

Often feel angry when think about health

Feel hopeless because of health problems

I get upset when think about my health
172

\%

77.4

78.0

71.6

53.8

82.7

95.2

82.2

88.9

87.5

47.1

21.6

18.8

12.5

19.7 
Table 15 (continued).

I get depressed if I think about my health

Self-Monitoring and Insight

As well as seeing doctor, regularly monitor changes in my health

I know what triggers and makes health problems worse

Have very good understanding of when/why I should take my meds

Have clear understanding of what to do to control health problems Have realistic expectations of what can and cannot do with health in mind

Constructive Attitudes and Approaches

Try not to let health problems stop from enjoying life

My health problems do not ruin my life

Feel well looked after by friends/family

Do not let health problems control life

$196 \quad 94.2$

If others can cope with problems like mine, I can too

Have effective ways to prevent symptoms from limiting what can do

Have clear understanding of equipment that could make life easier

Social Integration and Support

Have plenty of people can rely on if need help

Have enough friends who help me cope with health problems

Family and carers really understand when I feel ill

Feel well looked after by friends or family

$193 \quad 92.8$

Get enough chances to talk about health problems with people who understand me

Health Service Navigation

Have very positive relationships with my healthcare professionals

Communicate very confidently with my doctor about my healthcare needs

Confidently give healthcare professionals the information they need

to help me

Get needs met from available healthcare resources

96.6

I work in a team with doctors and other healthcare professionals

186

89.4

Notes. SD=standard deviation. Scales were scored based on developer guidelines, which required over $50 \%$ item completion per subscale for valid response scale scoring.

\subsubsection{Indicators of negative health behaviors}

Table 16 displays data describing indicators of potentially modifiable negative health behaviors.

Approximately $50 \%$ of the sample reported drinking alcoholic beverages within the past

30 days. The sample mean raw score for alcohol use, with negative alcohol screens set equal to zero, was 3.6. For individuals who screened positive for alcohol use, the mean sample T-score was $43.7(\mathrm{SD}=5.0$, range 38-59.4). Because T-scores are a standardized score with a mean of 
50 and a standard deviation of 10 (Patient-Reported Outcomes Measurement Information System, 2014), participants with negative alcohol consumption screens were not assigned a Tscore.

Approximately $53 \%$ of participants reported that they had smoked 100 cigarettes in their lifetime. Of those 109 individuals, the mean age at first cigarette was 15.6 years (range 6-30 years). The average pack year history, with nonsmokers equal to zero, was around 16 packyears (range 0-150). Around 9\% of respondents were current or recent (within the past 12 months) smokers, however, $44 \%(n=91)$ were past smokers.

The average BMI in this sample was $29.4 \mathrm{~kg} / \mathrm{m}^{2}$ (range 16.6-52.9). Because BMI has been shown to be associated with a J-shaped dose response curve for mortality (Aune et al., 2016), categorical data are also presented. Less than $3 \%$ of the sample was underweight. Around $25 \%$ were normal weight. Over $32 \%$ were overweight, and over $40 \%$ were obese. 
Table 16

Sample Characterization of Modifiable Health Behaviors $(N=211)$

\begin{tabular}{|c|c|c|}
\hline \multirow[b]{2}{*}{ Concept } & \multicolumn{2}{|c|}{ Result } \\
\hline & Mean (SD) or $\mathrm{n}$ & $\%$ or range \\
\hline \multicolumn{3}{|c|}{ Alcohol Use } \\
\hline Alcohol Use Raw Score $(n=211)$ & $3.6(4.0)$ & $0-16$ \\
\hline \multicolumn{3}{|l|}{$\begin{array}{l}\text { Drank Alcoholic Beverages within Past } 30 \text { Days } \\
(n=211)\end{array}$} \\
\hline No & 106 & 50.2 \\
\hline Yes & 105 & 49.8 \\
\hline Alcohol Use T-Score for Positive Screens $(n=105)$ & $43.7(5.0)$ & $38-59.4$ \\
\hline \multicolumn{3}{|c|}{ Tobacco Use } \\
\hline \multicolumn{3}{|l|}{ Smoked 100 Cigarettes in Lifetime $(n=208)$} \\
\hline No & 95 & 45.7 \\
\hline Yes & 110 & 52.9 \\
\hline If Yes, Age at First Cigarette $(n=109)$ & $15.6(3.5)$ & $6-30$ \\
\hline Pack Year History $(n=205)$ & $15.7(26.4)$ & $0-150$ \\
\hline \multicolumn{3}{|l|}{ Current Smoking Status $(n=207)$} \\
\hline Non-Smoker & 95 & 45.9 \\
\hline Past Smoker & 91 & 44.0 \\
\hline Current or Recent Smoker & 18 & 8.7 \\
\hline \multicolumn{3}{|c|}{ Anthropometric Data } \\
\hline Most Recently Documented BMI (kg/m²) (n=209) & $29.4(7.0)$ & $16.6-52.9$ \\
\hline \multicolumn{3}{|l|}{ Most Recent BMI by Category $(n=209)$} \\
\hline$\leq 18.5$ (Underweight) & 5 & 2.4 \\
\hline $18.5-24.9$ (Normal) & 62 & 24.9 \\
\hline 25.0-29.9 (Overweight) & 67 & 32.1 \\
\hline$>30$ (Obese) & 85 & 40.7 \\
\hline
\end{tabular}

Note. SD=standard deviation.

\subsection{DISCUSSION}

This study describes positive self-management behaviors and indicators of negative health behaviors in a sample of MPC survivors. While self-management behaviors were generally high there was variability among scores. Tobacco use findings were comparable to population norms, but rates of obesity were higher than both cancer and general populations.

Our analyses of self-management results were consistent with previous literature identifying MPC patients as more likely to be physically inactive than single cancer patients (Burris \& Andrykowski, 2011). Across items, about half to three quarters of the sample agreed that they were engaging in health directed activities, with walking for exercise only agreed upon 
by about half of the sample. Our finding that most of the sample agreed with activities reflective of self-monitoring and insight was also consistent with previous studies noting greater health awareness and positive healthcare utilization (i.e., attending regular appointments, monitoring for cancer, and current with cancer screenings) in MPC patients versus single cancer patients (Belcher et al., 2016; Thong et al., 2013). Less than $80 \%$ of participants felt that family and caregivers understood what they were going through when feeling ill, which is in line with previous literature finding greater interference with social activities in MPC patients than in single cancer patients (Thong et al., 2013), as measured by the Impact of Cancer scale (Costa et al., 2016). As identified in Section 3.0, BMl (overweight vs normal weight and obesity), graduate education, lower neuroticism, and increased social support were identified as characteristics associated with improved self-management and should be used to identify MPC patients at risk for poor self-management.

The National Institute for Nursing Research (NINR) describes the science of selfmanagement as examining strategies to aid understanding and management of one's illness and improve health behaviors (National Institute of Nursing Research, n.d.). The topic of selfmanagement has generated a great deal of interest in oncology over the past five years, with influence from a long history of research in chronic illness. Noted issues within selfmanagement science have included need for greater conceptual clarity, identification of valid measures, and identification of mechanisms (Grady et al., 2014). There is a great deal of debate as to how to best include self-management in oncology research and translate findings into practice. Current recommendations for common data elements (CDE) to use in studies of self-management include measures of activation (Patient Activation Measure ${ }^{\circledR}$ ), self-regulation (Index of Self-Regulation), self-efficacy for managing chronic conditions (Self-efficacy for Managing Chronic IIInesses Scale), global health (Patient-Reported Outcomes Measurement Information System [PROMIS] Global Heath short form), and biomarkers based on NINR symptom priorities of fatigue, depression, cognition, pain, and sleep disturbance (cytokines, 
HPA axis marker, neuropeptide, and DNA polymorphisms) (Moore et al., 2016; Page et al., 2018).

In contrast to the CDE recommended by NINR, which are limited to measures associated with health behaviors, the internationally-tested heiQ, selected to measure selfmanagement behaviors in this study, was designed specifically to measure outcomes following health education and self-management programs (Elsworth et al., 2015; Osborne et al., 2011, 2007) and directly addresses the range of self-management domains recommended to maintain wellness, including: health directed activity; positive and active engagement in life; emotional distress; self-monitoring and insight; constructive attitudes and approaches; skill technique and acquisition; social integration and support; and health service navigation. Future selfmanagement studies and nursing scientists should continue to study the best measures of selfmanagement, with consideration for whether the study intent is to measure the actual selfmanagement behaviors or the variables that are associated with self-management behaviors.

Our findings that approximately half of the sample reported drinking alcoholic beverages within the past 30 days is lower than the national rate (56.0\%) (National Institute on Alcohol Abuse and Alcoholism, 2017). Other literature using Behavioral Risk Factor Surveillance System data (Burris \& Andrykowski, 2011) has described higher alcohol consumption among MPC survivors who consume alcohol, based on analysis of typical number of drinks consumed in the past month when drinking. However, the effect sizes for these comparisons were small, and, as authors acknowledge, are not likely to be clinically meaningful.

Smoking is the number one preventable cause of death in the U.S. and increases risk for heart disease, stroke, and cancer (Center for Disease Control and Prevention, 2018). Tobacco smoking is a known carcinogen in the general population and has been implicated as a causative factor associated with increased risk for additional cancer development in cancer survivors (Fraumeni Jr et al., 2006); length and duration of exposure are known to impact carcinogenesis (National Cancer Institute, 2015). Only around $9 \%$ of our study participants were 
current or recent smokers; however, $44 \%$ were past smokers. Of these individuals, the mean age at first cigarette was 15.6 (range: 6-30 years), and pack year history ranged from 0-150 (Mean=16). When compared to national and regional data, the rates of current smoking in this sample was lower than the national average, similar to rates in adults 65 years of age and older, and lower than rates in Pennsylvania (9\% versus $15.5 \%$ and $18.6-21.7 \%$, respectively) (The Centers for Disease Control and Prevention, 2018; Underwood et al., 2012). Both the early age at which some of our participants first smoked (i.e., 6 years old) and the high pack year history (i.e., 150) are notable. Future studies should evaluate triggers for smoking cessation in patients with MPC.

Obesity is a risk factor for many diseases and conditions [e.g., heart disease, osteoarthritis, sleep apnea, depression, anxiety, pain, physical function impairment), including being a risk factor for cancer (i.e., breast [post-menopausal women], endometrial, colon, kidney, gallbladder, and liver) (Centers for Disease Control and Prevention, 2015). Additionally, diet, physical inactivity, and obesity are implicated as potential risk factors for subsequent cancer development among survivors of breast (female), reproductive organs, and upper and lower digestive tract cancers (Fraumeni et al., 2006). The high rates of obesity in this sample (over $40 \%$ ) is concerning, given the associated known health risks. The American Society of Clinical Oncology and other organizations have published position statements on obesity and cancer offer strategies for healthcare providers to address obesity with their patients (Ligibel et al., 2014).

Previous studies have suggested greater health awareness in MPC versus single cancer patients (Belcher et al., 2017; Thong et al., 2013). However, awareness does not directly translate to behavioral change. A cancer diagnosis or experience has been described as a teachable moment, but studies have pointed out that desire to change varies by individuals and is impacted by many complex factors (Corbett et al., 2018; Demark-Wahnefried, Aziz, Rowland, \& Pinto, 2005). Others have suggested that the teachable moment afforded during one's cancer 
experience may be halted by subsequent cancer diagnoses (Burris \& Andrykowski, 2011). Future research should evaluate how having more than one cancer impacts health behavior changes.

In addition to the poorer health observed in patients with MPC (Andrykowski, 2012; Belcher et al., 2017; Burris \& Andrykowski, 2011; Dowling et al., 2013; Gotay et al., 2007; Thong et al., 2013), some MPC patients (16\% in this study) will go on to develop more than two primary cancers. In addition to non-modifiable familial cancer syndromes and potential carcinogenetic effects of previous cancer treatments, risk for development of more than one primary cancer diagnosis increases with modifiable causative exposures (e.g., smoking and alcohol); diet, obesity, physical inactivity, and reproductive risk factors have also been implicated in the development of subsequent cancers in patients with breast (female), reproductive, and upper and lower digestive tract cancers (Fraumeni et al., 2006; Schottenfeld \& Beebe-Dimmer, 2006). Health optimization and risk reduction remain critical in this subset of cancer survivors; future MPC research should include self-management as a modifiable target through which this may be achieved. 


\subsection{APPENDICES}


APPENDIX A

STUDY RECRUITMENT MATERIALS AND REFUSAL FORM 


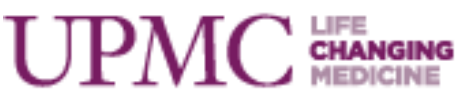

October 11, 2016

Sarah M. Belcher, RN, BSN, OCN

Predoctoral Scholar

Robert Wood Johnson Foundation Future of Nursing Scholars

Health \& Community Systems, University of Pittsburgh School of Nursing

3500 Victoria Street

Room 434 Victoria Building

Pittsburgh, PA 15261

Dear Sarah,

This letter is to formally confirm the number of patients in the UPMC Network Cancer Registry who fit the criteria for the human subjects portion of the American Cancer Society Doctoral Degree Scholarship in Cancer Nursing application that you are submitting entitled "Characterizing Psychobehavioral Risks in Multiple Primary Cancer Survivors."

As you are aware, the UPMC Network Cancer Registry maintains a standardized data system designed for the collection, management, and analysis of patient demographic, grading, staging, treatment and progression data on patients having a diagnosis of cancer who are treated at UPMC hospitals and hospital based clinics of UPMC CancerCenters. Given the criteria you provided (e.g. Include: adults [18 or older]; all cancer diagnosed during adulthood; history of 2 or more primary cancers; only Stage I-III cancers; all UPMC sites; and all years of registry inclusiveness. Exclude: cases of non-melanoma skin cancer; in situ cancers; Stage IV/M1 cases at presentation; and disease recurrent cases [same as original cancer diagnosis]), there is expected to be an sufficient pool of potential participants (5,757 survivors) to assure that you will be able to attain your target sample size.

Should you have any questions about the data provided by the UPMC Network Cancer Registry and our Registry Information Services, or should you need additional data, please feel free to contact me.

Sincerely,

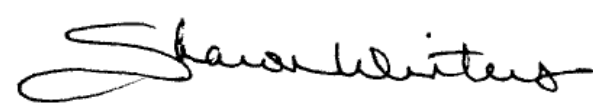

Sharon Winters, MS, CTR

Director, Registry Information Services, UPMC CancerCenter

Manager, HSTB/RIS/DBMI Collaborative Honest Broker System (IRB\#HB015)

Adjunct Instructor, University of Pittsburgh

Shadyside Place

First Floor, Suite 110

580 South Aiken Avenue

Pittsburgh, PA 15232Pittsburgh, PA 15232-1304

(412) 647-6390

winterssb@upmc.edu 
Dear [Title] [Patient Name],

I'm writing to tell you about an exciting new research study called Shining a light on Life After Multiple Primary cancers: The LAMP study. This research study is led by Sarah Belcher, a nurse and graduate student at the University of Pittsburgh School of Nursing.

We want to learn more about patients who have had more than one cancer. You have been identified through UPMC's tumor registry as someone who may be eligible for this research study.

If you choose to participate, you will be asked to provide consent to participate in the research study. You will then fill out a one-time survey (30-40 minutes) online or through the mail. This survey asks about your stress, health behaviors, emotions, and overall health. With your permission, we will then combine your responses with health and cancer treatment information from your medical record. We will use this information to better understand and better support cancer survivors like you. You will receive a $\$ 5$ Amazon gift card for completing the survey.

You can complete the survey in a couple of different ways:

1. You can easily complete the consent and survey online by typing the following website URL into your browser: http://tinyurl.com/LAMP-PittNursing

2. If you would rather have a paper copy, please complete and return the enclosed Paper Survey Request Form in the pre-paid envelope included with this letter.

If you do not wish to be in the study, you can complete a voluntary refusal form either online at http://tinyurl.com/LAMP-PittNursing or via the enclosed paper Refusal Form.

Please call or email the study lead, Sarah Belcher, with any questions at:

- 412-624-8938 or

- LAMPstudy@pitt.edu

This research study is funded by the American Cancer Society, the Robert Wood Johnson Foundation Future of Nursing Scholars program, the Nightingale Awards of Pennsylvania, and the School of Nursing.

I fully support this study and believe it will help us to better meet the needs of cancer survivors like you. I hope you will take the time to participate in this important research study.

Sincerely, 


\section{University of Pittsburgh}

\section{Paper Survey Request Form}

Thank you for your interest in the LAMP study!

Please fill out the information below, and return this form in the included postage-paid envelope. We will mail you a paper copy of the survey.

I am interested in completing questionnaires on paper.

My name is:

My mailing address is:

My email address is:

Thank you for your time! If you have any questions, please contact Sarah Belcher at 412-624-8938 or LAMPstudy@pitt.edu.

ID:

(for study use only) 


\section{University of Pittsburgh}

\section{Refusal Form}

We understand that not everyone is able or interested in completing the survey. We are trying to understand more about the people who do not complete the survey. If you are willing, it would help us if you would answer and return the following 5 general questions about yourself in the included prepaid envelope. These answers are confidential and voluntary.

If you prefer, this information may also be completed online at:

\section{http://tinyurl.com/LAMP-PittNursing}

Instructions: Please circle the answers that best describe you.

1. What is your sex?
a. Male
b. Female

2. How long has it been since your most recent cancer treatment?
a. 0-1 year
b. 1-5 years
c. 5-10 years
d. Over 10 years
e. I am currently receiving treatment for cancer.

3. What best describes your race? Select all that apply.
a. White or Caucasian
b. African American
c. American Indian
d. Alaska Native
e. Native Hawaiian or other Pacific Islander
f. Asian
g. Unknown
h. Other

4. What is the highest grade or degree you have completed?
a. Less than High School
b. High school diploma or GED
c. 2 year/ Associate's Degree
d. 4 year/Bachelor's Degree
e. Graduate/Professional Degree (Masters, PhD, MD, JD, etc.)

5. What age group best describes you:
a. $18-29$
b. $30-49$
c. $50-69$
d. 70 and above 
Can you briefly share why you don't want to participate in this study?

Is there anything else you would like us to know?

Thank you for your time!

Please return this form in the postage-paid envelope included with this mailing.

If you have any questions, please contact Sarah Belcher at 412-624-8938 or LAMPstudy@pitt.edu.

ID:

(for study use only) 


\section{University of Pittsburgh School of Nursing}

You may have recently received a letter inviting you to participate in The LAMP study, a research study led by a nurse and graduate student at the University of Pittsburgh School of Nursing.

Thank you if you already responded! If you have not yet responded and would like more information about this one-time study, please contact us via the phone or email information listed below. You can also log on to the following website to complete the one-time survey or a refusal form online: http://tinyurl.com/LAMP-PittNursing

Please call or email the study lead, Sarah Belcher, with any questions or to request a paper survey copy. 412-624-8938 | LAMPstudy@pitt.edu

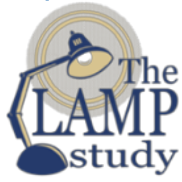

\section{University of Pittsburgh School of Nursing}

You may have recently received a letter inviting you to participate in The LAMP study, a research study led by a nurse and graduate student at the University of Pittsburgh School of Nursing.

Thank you if you already responded! If you have not yet responded and would like more information about this one-time study, please contact us via the phone or email information listed below. You can also log on to the following website to complete the one-time survey or a refusal form online: http://tinyurl.com/LAMP-PittNursing

Please call or email the study lead, Sarah Belcher, with any questions or to request a paper survey copy. 412-624-8938 | LAMPstudy@pitt.edu
University of Pittsburgh School of Nursing

You may have recently received a letter inviting you to participate in The LAMP study, a research study led by a nurse and graduate student at the University of Pittsburgh School of Nursing.

Thank you if you already responded! If you have not yet responded and would like more information about this one-time study, please contact us via the phone or email information listed below. You can also log on to the following website to complete the one-time survey or a refusal form online: http://tinyurl.com/LAMP-PittNursing

Please call or email the study lead, Sarah Belcher, with any questions or to request a paper survey copy. 412-624-8938 | LAMPstudy@pitt.edu

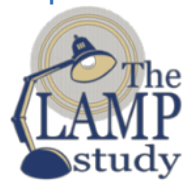

\section{University of Pittsburgh School of Nursing}

You may have recently received a letter inviting you to participate in The LAMP study, a research study led by a nurse and graduate student at the University of Pittsburgh School of Nursing.

Thank you if you already responded! If you have not yet responded and would like more information about this one-time study, please contact us via the phone or email information listed below. You can also log on to the following website to complete the one-time survey or a refusal form online: http://tinyurl.com/LAMP-PittNursing

Please call or email the study lead, Sarah Belcher, with any questions or to request a paper survey copy. 412-624-8938 | LAMPstudy@pitt.edu
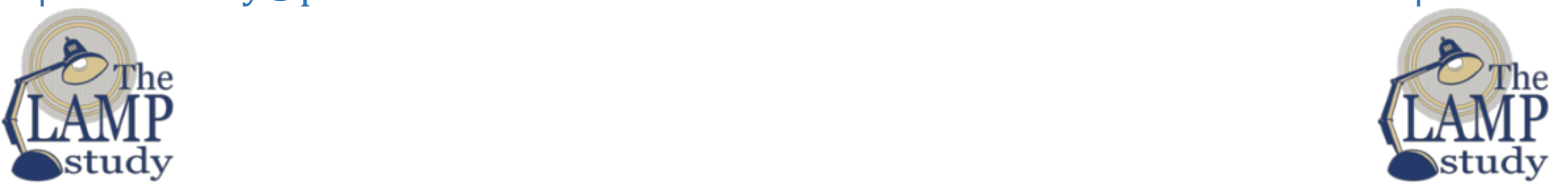


\section{The LAMP Study}

University of Pittsburgh School of Nursing

415 Victoria Building

3500 Victoria Street

Pittsburgh, PA 15261

[Recipient Name]

[Street Address]

[Address 2]

[City, ST ZIP Code]

\section{The LAMP Study}

University of Pittsburgh School of Nursing

415 Victoria Building

3500 Victoria Street

Pittsburgh, PA 15261

[Recipient Name]

[Street Address]

[Address 2]

[City, ST ZIP Code]
The LAMP Study

University of Pittsburgh School of Nursing

415 Victoria Building

3500 Victoria Street

Pittsburgh, PA 15261

\section{The LAMP Study}

University of Pittsburgh School of Nursing

415 Victoria Building

3500 Victoria Street

Pittsburgh, PA 15261
[Recipient Name]

[Street Address]

[Address 2]

[City, ST ZIP Code]
[Recipient Name] [Street Address]

[Address 2]

[City, ST ZIP Code] 


\section{University of Pittsburgh School of Nursing}

Thank you for your interest in The LAMP study! We recently sent you the survey via mail. Thank you if you already returned it!

If you have not yet completed the survey, this is a friendly reminder to complete and return it in the included postage-paid envelope. If you did not receive your survey packet or would like more information, please contact us via the phone or email contacts listed below. The survey can also be completed online at:

http://tinyurl.com/LAMP-PittNursing

Please call or email the study lead, Sarah Belcher, with any questions or to request a replacement survey. 412-624-8938 | LAMPstudy@pitt.edu

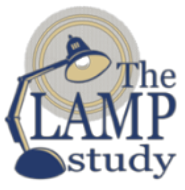

\section{University of Pittsburgh School of Nursing}

Thank you for your interest in The LAMP study! We recently sent you the survey via mail. Thank you if you already returned it!

If you have not yet completed the survey, this is a friendly reminder to complete and return it in the included postage-paid envelope. If you did not receive your survey packet or would like more information, please contact us via the phone or email contacts listed below. The survey can also be completed online at:

\section{http://tinyurl.com/LAMP-PittNursing}

Please call or email the study lead, Sarah Belcher, with any questions or to request a paper survey copy. 412-624-8938 | LAMPstudy@pitt.edu

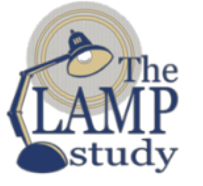

\section{University of Pittsburgh School of Nursing}

Thank you for your interest in The LAMP study! We recently sent you the survey via mail. Thank you if you already returned it!

If you have not yet completed the survey, this is a friendly reminder to complete and return it in the included postage-paid envelope. If you did not receive your survey packet or would like more information, please contact us via the phone or email contacts listed below. The survey can also be completed online at:

\section{http://tinyurl.com/LAMP-PittNursing}

Please call or email the study lead, Sarah Belcher, with any questions or to request a paper survey copy. 412-624-8938 | LAMPstudy@pitt.edu

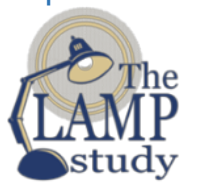

\section{University of Pittsburgh School of Nursing}

Thank you for your interest in The LAMP study! We recently sent you the survey via mail. Thank you if you already returned it!

If you have not yet completed the survey, this is a friendly reminder to complete and return it in the included postage-paid envelope. If you did not receive your survey packet or would like more information, please contact us via the phone or email contacts listed below. The survey can also be completed online at:

\section{http://tinyurl.com/LAMP-PittNursing}

Please call or email the study lead, Sarah Belcher, with any questions or to request a paper survey copy. 412-624-8938 | LAMPstudy@pitt.edu 
The LAMP Study

University of Pittsburgh

School of Nursing

415 Victoria Building

3500 Victoria Street

Pittsburgh, PA 15261

\section{The LAMP Study}

University of Pittsburgh

School of Nursing

415 Victoria Building

3500 Victoria Street

Pittsburgh, PA 15261
The LAMP Study

University of Pittsburgh

School of Nursing

415 Victoria Building

3500 Victoria Street

Pittsburgh, PA 15261

\section{The LAMP Study}

University of Pittsburgh

School of Nursing

415 Victoria Building

3500 Victoria Street

Pittsburgh, PA 15261 
APPENDIX B

STUDY COVER LETTER AND INSTRUMENTS 


\section{University of Pittsburgh}

School of Nursing

Department of Health \& Community Systems

Thank you so much for your interest in volunteering to participating in the LAMP research study. As you may know, I am an oncology nurse and graduate student at the University of Pittsburgh School of Nursing. I am conducting this study as part of my dissertation work to better understand and support cancer survivors like you who have had two separate types of cancer. We call these types of cancers, "multiple primary cancers."

First, please review and complete one copy of the Consent Form included with this mailing. The signed consent form must be returned along with your survey responses in order for us to include you in the study. A second copy is provided for you to keep for yourself.

The study consists of two parts:

1. We ask you to complete and return the included paper survey questionnaire packet and return it along with the Consent Form in the included prepaid envelope.

The questions will ask about your stress, health behaviors, emotions, and overall health. This survey will take you about $30-40$ minutes to complete. We realize that we are asking you for a significant amount of time to complete the questions. If you need a break, you can pause and come back to the survey later. We truly believe that the experiences of people like you who have had multiple primary cancers have not be adequately been addressed in research and clinical practice. It is our hope that your participation in this study will help us address this critical lack of understanding and help people like you in the future.

2. Next, our research team will review your medical records to find out more about your cancer and health history.

You will receive a $\$ 5$ Amazon.com gift card code for completing the survey.

Please note that your responses to this survey will not be sent to your healthcare providers. It is important that you contact or see your professional healthcare team if you have any questions or concerns about physical or emotional symptoms, cancer, or medications. If you experience a new symptom or increase in severity of an existing symptom, please report this to your healthcare team or emergency medical services immediately.

Each questionnaire has a unique set of instructions and possible response options. Questions will ask you to think back about different periods of time. Some questions may seem similar, because we are testing different ways of asking about similar ideas. There are no right or wrong answers.

If you have questions at any point, you can contact me, Sarah Belcher, at LAMPstudy@pitt.edu or 412-624-8938.

Sincerely,

Sarah M. Belcher, $\mathrm{PhD}(\mathrm{c}), \mathrm{RN}, \mathrm{OCN}{ }^{\circledR}$ 


\section{Shining a light on Life After Multiple Primary cancers}

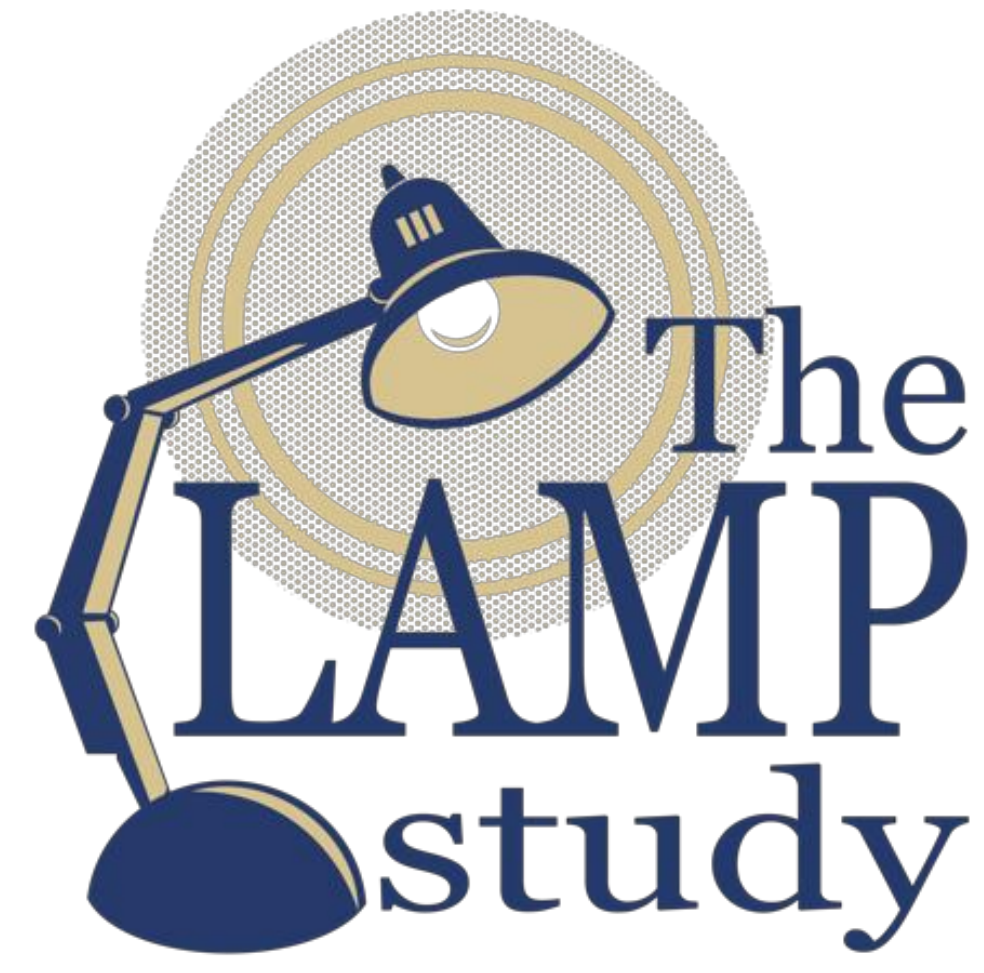

\section{LAMP Study Questionnaires}

\section{Thank you for being a part of this study!}

Please return this completed packet and a copy of the signed consent and to Sarah Belcher in the enclosed postage-paid envelope.

ID:

(for study use only) 


\section{Socio-Demographic Questionnaire - Part 1}

Please begin by entering the following basic information about yourself.

SD_1. First Name:

SD_2. Middle Initial:

SD_3. Last Name:

SD_4. Date of birth:

$\overline{\text { (month }}^{\prime} / \overline{\text { day }} /^{\prime}$ year)

SD_5. Current Age in years: years old
SD_6. What is your sex?
- Male
- Female

SD_7. Do you consider yourself to be Hispanic or Latino? That is, of Mexican, Puerto Rican, Cuban, Caribbean, or of Latin American descent.
- Yes
- No
- Do not know

SD_8. Please select the racial and ethnic category or categories with which you most closely identify. Check all that apply.

- White or Caucasian

- Black or African American

- American Indian (SD8a. specify tribe )

- Alaska Native

- Native Hawaiian or other Pacific Islander

- Asian

- Unknown

- Other (SD8b. specify 


\section{(socio-demographics, continued)}

SD 9. Email Address:

SD_10. Phone Number: ) $-$

SD_11. Does your current household income meet your basic needs?

- Yes

- No 


\section{Multiple Primary Cancer (MPC) Items - Part 1}

In order to provide the best care and support, we need to understand how a second cancer diagnosis may be different than a first cancer diagnosis. Thank you for taking the time to share your experiences with us.

MPC_1. How old were you when you were first diagnosed with cancer? years old

MPC_2. What type of cancer were you first diagnosed with?

MPC_3. How old were you when you were diagnosed with a second type of cancer? years old

MPC_4. What type of cancer was your second cancer diagnosis?

MPC_5. Were you diagnosed with any additional cancers? For example, a third or more different type of cancer.

- No $\rightarrow$ Skip to MPC_6 on page 4 .

- Yes

MPC_5a. If YES, please explain: 


\section{(MPC items, continued)}

MPC_6. What is your current stage of cancer survivorship? Please indicate with a check all that apply.

- I finished treatment less than 1 year ago.

- I finished treatment between 1 and 5 years ago.

- I finished treatment between 5 and 10 years ago.

- I finished treatment 10 or more years ago.

- I am currently receiving treatment for cancer.

- I am living with cancer as a chronic illness.

- I am currently receiving palliative care.

- I am currently receiving hospice care.

- I prefer not to answer, or I am not sure. 


\section{(MPC items, continued)}

MPC_7. For each of the following common challenges or stressors, please select whether your second cancer diagnosis was more difficult, the same, or less difficult than your first cancer diagnosis. Select N/A if this was not a problem with either cancer diagnosis.

\begin{tabular}{|c|c|c|c|c|}
\hline & $\begin{array}{l}\text { This was more } \\
\text { difficult with my } \\
\text { second cancer. }\end{array}$ & $\begin{array}{l}\text { This was the } \\
\text { same for } \\
\text { both cancers. }\end{array}$ & $\begin{array}{l}\text { This was less } \\
\text { difficult with my } \\
\text { second cancer. }\end{array}$ & $\begin{array}{l}\text { N/A - This was } \\
\text { not a problem for } \\
\text { me with either } \\
\text { cancer diagnosis. }\end{array}$ \\
\hline MPC_7a. Managing stress & $\square$ & $\square$ & $\square$ & 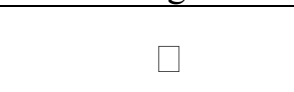 \\
\hline MPC_7b. Feeling down or blue & $\square$ & $\square$ & $\square$ & $\square$ \\
\hline $\begin{array}{l}\text { MPC_7c. Feeling nervous or } \\
\text { anxious }\end{array}$ & $\square$ & $\square$ & $\square$ & $\square$ \\
\hline $\begin{array}{l}\text { MPC_7d. Managing my overall } \\
\text { health }\end{array}$ & $\square$ & $\square$ & $\square$ & $\square$ \\
\hline $\begin{array}{l}\text { MPC_7e. Finding good ways to } \\
\text { cope }\end{array}$ & $\square$ & $\square$ & $\square$ & $\square$ \\
\hline $\begin{array}{l}\text { MPC_7f. Completing life activities } \\
\text { (e.g., bathing/dressing, } \\
\text { light housework, walking } \\
\text { more than a mile) }\end{array}$ & $\square$ & $\square$ & $\square$ & $\square$ \\
\hline $\begin{array}{l}\text { MPC_7g. Treatment- and/or } \\
\text { cancer-related symptoms }\end{array}$ & $\square$ & $\square$ & $\square$ & $\square$ \\
\hline MPC_7h. Financial hardship & $\square$ & $\square$ & $\square$ & $\square$ \\
\hline $\begin{array}{c}\text { MPC_7i. Managing my } \\
\text { relationships }\end{array}$ & $\square$ & $\square$ & $\square$ & $\square$ \\
\hline $\begin{array}{l}\text { MPC_7j. Getting the support that I } \\
\text { need }\end{array}$ & $\square$ & $\square$ & $\square$ & $\square$ \\
\hline $\begin{array}{c}\text { MPC_7k. Communicating with my } \\
\text { healthcare team }\end{array}$ & $\square$ & $\square$ & $\square$ & $\square$ \\
\hline $\begin{array}{l}\text { MPC_71. Other challenge or } \\
\text { stressor? - please specify: } \\
\text { MPC_7m. }\end{array}$ & $\square$ & $\square$ & $\square$ & $\square$ \\
\hline
\end{tabular}




\section{(MPC items, continued)}

MPC_8n. Would you like to further explain any of the answers you provided in the previous table?

- $\quad$ No $\rightarrow$ Skip to PSS_1 on page 7.

- Yes $\rightarrow$ MPC_8o. If YES, please explain in the space below: 


\section{General Stress}

The questions in this scale ask you about your feelings and thoughts during the last month. In each case, please indicate with a check how often you felt or thought a certain way.

\begin{tabular}{|c|c|c|c|c|c|}
\hline & Never & $\begin{array}{c}\text { Almost } \\
\text { never }\end{array}$ & $\begin{array}{l}\text { Some- } \\
\text { times }\end{array}$ & $\begin{array}{c}\text { Fairly } \\
\text { often }\end{array}$ & $\begin{array}{l}\text { Very } \\
\text { often }\end{array}$ \\
\hline $\begin{array}{l}\text { PSS_1. In the last month, how often have you } \\
\text { been upset because of something that } \\
\text { happened unexpectedly? }\end{array}$ & $\square$ & $\square$ & $\square$ & $\square$ & $\square$ \\
\hline $\begin{array}{l}\text { PSS_2. In the last month, how often have you felt } \\
\text { that you were unable to control the } \\
\text { important things in your life? }\end{array}$ & $\square$ & $\square$ & $\square$ & $\square$ & $\square$ \\
\hline $\begin{array}{l}\text { PSS_3. In the last month, how often have you felt } \\
\text { nervous and "stressed"? }\end{array}$ & $\square$ & $\square$ & $\square$ & $\square$ & $\square$ \\
\hline $\begin{array}{l}\text { PSS_4. In the last month, how often have you felt } \\
\text { confident about your ability to handle } \\
\text { your personal problems? }\end{array}$ & $\square$ & $\square$ & $\square$ & $\square$ & $\square$ \\
\hline $\begin{array}{l}\text { PSS_5. In the last month, how often have you felt } \\
\text { that things were going your way? }\end{array}$ & $\square$ & $\square$ & $\square$ & $\square$ & $\square$ \\
\hline $\begin{array}{l}\text { PSS_6. In the last month, how often have you } \\
\text { found that you could not cope with all } \\
\text { the things that you had to do? }\end{array}$ & $\square$ & $\square$ & $\square$ & $\square$ & $\square$ \\
\hline $\begin{array}{l}\text { PSS_7. In the last month, how often have you } \\
\text { been able to control irritations in your } \\
\text { life? }\end{array}$ & $\square$ & $\square$ & $\square$ & $\square$ & $\square$ \\
\hline $\begin{array}{l}\text { PSS_8. In the last month, how often have you felt } \\
\text { that you were on top of things? }\end{array}$ & $\square$ & $\square$ & $\square$ & $\square$ & $\square$ \\
\hline $\begin{array}{l}\text { PSS_9. In the last month, how often have you } \\
\text { been angered because of things that were } \\
\text { outside of your control? }\end{array}$ & $\square$ & $\square$ & $\square$ & $\square$ & $\square$ \\
\hline $\begin{array}{l}\text { PSS_10. In the last month, how often have you } \\
\text { felt difficulties were piling up so high } \\
\text { that you could not overcome them? }\end{array}$ & $\square$ & $\square$ & $\square$ & $\square$ & $\square$ \\
\hline
\end{tabular}




\section{Cancer-Related Stress}

Instructions - Below is a list of difficulties people sometimes have after a diagnosis of cancer. Please reach each item, and then indicate how distressing (or how common) each difficulty has been for you during the past seven days.

(check one box on each line)

\begin{tabular}{|c|c|c|c|c|c|}
\hline & $\begin{array}{c}\text { Not at } \\
\text { all } \\
0 \\
\end{array}$ & $\begin{array}{c}\text { A little } \\
\text { bit } \\
1 \\
\end{array}$ & $\begin{array}{c}\text { Moder- } \\
\text { ately } \\
2 \\
\end{array}$ & $\begin{array}{c}\text { Quite a } \\
\text { bit } \\
3 \\
\end{array}$ & $\begin{array}{c}\text { Extreme } \\
-1 y \\
4 \\
\end{array}$ \\
\hline $\begin{array}{l}\text { IES_1. Any reminder of cancer brought back feelings } \\
\text { about it. }\end{array}$ & $\square$ & $\square$ & $\square$ & $\square$ & $\square$ \\
\hline IES_2. I had trouble staying asleep. & $\square$ & $\square$ & $\square$ & $\square$ & $\square$ \\
\hline $\begin{array}{l}\text { IES_3. Other things kept making me think about } \\
\text { cancer. }\end{array}$ & $\square$ & $\square$ & $\square$ & $\square$ & $\square$ \\
\hline IES_4. I felt irritable and angry. & $\square$ & $\square$ & $\square$ & $\square$ & $\square$ \\
\hline $\begin{array}{l}\text { IES_5. I avoided letting myself get upset when I } \\
\text { thought about cancer or was reminded of it. }\end{array}$ & $\square$ & $\square$ & $\square$ & $\square$ & $\square$ \\
\hline IES_6. I thought about cancer when I didn't mean to. & $\square$ & $\square$ & $\square$ & $\square$ & $\square$ \\
\hline IES_7. I felt as if it hadn't happened or wasn't real. & $\square$ & $\square$ & $\square$ & $\square$ & $\square$ \\
\hline IES_8. I stayed away from reminders about cancer. & $\square$ & $\square$ & $\square$ & $\square$ & $\square$ \\
\hline $\begin{array}{l}\text { IES_9. Pictures about being ill with cancer popped } \\
\text { into my mind. }\end{array}$ & $\square$ & $\square$ & $\square$ & $\square$ & $\square$ \\
\hline IES_10. I was jumpy and easily startled. & $\square$ & $\square$ & $\square$ & $\square$ & $\square$ \\
\hline IES_11. I tried not to think about cancer. & $\square$ & $\square$ & $\square$ & $\square$ & $\square$ \\
\hline $\begin{array}{l}\text { IES_12. I was aware that I still had a lot of feelings } \\
\text { about cancer, but I didn't deal with them. }\end{array}$ & $\square$ & $\square$ & $\square$ & $\square$ & $\square$ \\
\hline $\begin{array}{l}\text { IES_13. My feelings about cancer were kind of } \\
\text { numb. }\end{array}$ & $\square$ & $\square$ & $\square$ & $\square$ & $\square$ \\
\hline $\begin{array}{l}\text { IES_14. I found myself feeling as though I was back } \\
\text { at that time of my bad news. }\end{array}$ & $\square$ & $\square$ & $\square$ & $\square$ & $\square$ \\
\hline IES_15. I had trouble falling asleep. & $\square$ & $\square$ & $\square$ & $\square$ & $\square$ \\
\hline IES_16. I had waves of strong feelings about cancer. & $\square$ & $\square$ & $\square$ & $\square$ & $\square$ \\
\hline IES_17. I tried to remove cancer from my memory. & $\square$ & $\square$ & $\square$ & $\square$ & $\square$ \\
\hline IES_18. I had trouble concentrating. & $\square$ & $\square$ & $\square$ & $\square$ & $\square$ \\
\hline
\end{tabular}


(cancer-related stress, continued)

\begin{tabular}{|l|c|c|c|c|c|}
\hline & $\begin{array}{c}\text { Not at } \\
\text { all } \\
\mathbf{0}\end{array}$ & $\begin{array}{c}\text { A little } \\
\text { bit } \\
\mathbf{1}\end{array}$ & $\begin{array}{c}\text { Moderate } \\
\text {-ly } \\
\mathbf{2}\end{array}$ & $\begin{array}{c}\text { Quite a } \\
\text { bit } \\
\mathbf{3}\end{array}$ & $\begin{array}{c}\text { Extreme- } \\
\text { ly } \\
\mathbf{4}\end{array}$ \\
\hline $\begin{array}{l}\text { IES_19. Reminders of cancer caused me to have } \\
\text { physical reactions, such as sweating, } \\
\text { trouble breathing, nausea, or a pounding } \\
\text { heart. }\end{array}$ & $\square$ & $\square$ & $\square$ & $\square$ & $\square$ \\
\hline IES_20. I had dreams about cancer. & $\square$ & $\square$ & $\square$ & $\square$ & $\square$ \\
\hline IES_21. I felt watchful or on-guard. & $\square$ & $\square$ & $\square$ & $\square$ & $\square$ \\
\hline IES_22. I tried not to talk about cancer. & $\square$ & $\square$ & $\square$ & $\square$ & $\square$ \\
\hline
\end{tabular}




\section{Cancer Survivor Concerns}

Below is a list of worries people sometimes have after a diagnosis of cancer. Please indicate how much worry you experience with each of the following topics.

\begin{tabular}{|l|c|c|c|c|}
\hline I worry about... & $\begin{array}{c}\text { Not at all } \\
\mathbf{1}\end{array}$ & $\begin{array}{c}\text { A little bit } \\
\mathbf{2}\end{array}$ & $\begin{array}{c}\text { Somewhat } \\
\mathbf{3}\end{array}$ & $\begin{array}{c}\text { Very much } \\
\mathbf{4}\end{array}$ \\
\hline ASC_1. Future diagnostic tests & $\square$ & $\square$ & $\square$ & $\square$ \\
\hline ASC_2. Another type of cancer & $\square$ & $\square$ & $\square$ & $\square$ \\
\hline $\begin{array}{l}\text { ASC_3. My cancer coming } \\
\text { back }\end{array}$ & $\square$ & $\square$ & $\square$ & $\square$ \\
\hline
\end{tabular}




\section{Emotions, part 1}

Over the last 2 weeks, how often have you been bothered by any of the following problems?

(check one box on each line)

How often during the past 2 weeks were you bothered by...

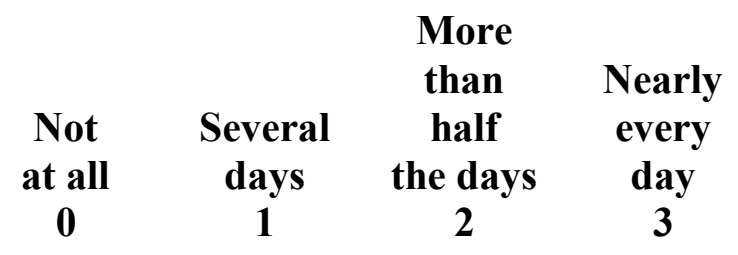

PHQ_1. Little interest or pleasure in doing things.

PHQ_2. Feeling down, depressed, or hopeless.

If you answered "Not at All" for BOTH PHQ_1 and PHQ_2 above, skip ahead now to page 12 (GAD_1). Otherwise, continue to $P H Q \_3$ below.

PHQ_3. Trouble falling or staying asleep, or sleeping too much.

PHQ_4. Feeling tired or having little energy.

PHQ_5. Poor appetite or overeating....

PHQ_6. Feeling bad about yourself, or that you are a failure, or have let yourself or your family down...............

PHQ_7. Trouble concentrating on things, such as reading the newspaper or watching television

PHQ_8. Moving or speaking so slowly that other people could have noticed. Or the opposite - being so fidgety or restless that you have been moving around a lot more than usual......... 


\section{Emotions, part 2}

\begin{tabular}{|c|c|c|c|c|}
\hline $\begin{array}{l}\text { Over the last } 2 \text { weeks, how often have you } \\
\text { been bothered by the following problems? } \\
\text { (check one box on each line) }\end{array}$ & $\begin{array}{l}\text { Not } \\
\text { at all } \\
\text { sure } \\
0\end{array}$ & $\begin{array}{c}\text { Several } \\
\text { days } \\
1\end{array}$ & $\begin{array}{c}\text { Over } \\
\text { half } \\
\text { the days } \\
2\end{array}$ & $\begin{array}{l}\text { Nearly } \\
\text { every day } \\
3\end{array}$ \\
\hline GAD_1. Feeling nervous, anxious, or on edge & $\square$ & $\square$ & $\square$ & $\square$ \\
\hline $\begin{array}{l}\text { GAD_2. Not being able to stop or control } \\
\text { worrying }\end{array}$ & $\square$ & $\square$ & $\square$ & $\square$ \\
\hline \multicolumn{5}{|c|}{$\begin{array}{l}\text { If you answered "Not at All" for BOTH GAD_1 and GAD_2 above, skip ahead now to } \\
\text { heiQ_1 on page 13. Otherwise, continue to } G A D_{-} 3 \text { below. }\end{array}$} \\
\hline $\begin{array}{l}\text { GAD_3. Worrying too much about different } \\
\text { things }\end{array}$ & $\square$ & $\square$ & $\square$ & $\square$ \\
\hline GAD_4. Trouble relaxing & $\square$ & $\square$ & $\square$ & $\square$ \\
\hline $\begin{array}{l}\text { GAD_5. Being so restless that it's hard to sit } \\
\text { still }\end{array}$ & $\square$ & $\square$ & $\square$ & $\square$ \\
\hline GAD_6. Becoming easily annoyed or irritable & $\square$ & $\square$ & $\square$ & $\square$ \\
\hline $\begin{array}{l}\text { GAD_7. Feeling afraid as if something awful } \\
\text { might happen }\end{array}$ & $\square$ & $\square$ & $\square$ & $\square$ \\
\hline
\end{tabular}

GAD_8. If you checked off any of the problems, how difficult have these made it for you to do your work, take care of things at home, or get along with other people?

Not difficult at all

Somewhat difficult

Very difficult

Extremely difficult 


\section{Self-Management of Your Health}

\section{Instructions}

There are no right or wrong answers but please make sure that you answer every question the best you can.

Please indicate how strongly you disagree or agree with the following statements by checking the response which best describes you now.

\section{Please answer the following questions:}

Check a box by crossing it:

Right now

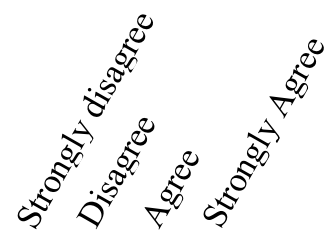

heiQ_1. On most days of the week, I do at least one activity to improve my health (e.g., walking, relaxation, exercise).

heiQ_2. Most days I am doing some of the things I really enjoy.

heiQ_3. As well as seeing my doctor, I regularly monitor changes in my health.

heiQ_4. I often worry about my health.

heiQ_5. I try to make the most of my life.

heiQ_6. I know what things can trigger my health problems and make them worse.

heiQ_7. My health problems make me very dissatisfied with my life.

heiQ_8. I am doing interesting things in my life.

heiQ_9. I do at least one type of physical activity every day for at least 30 minutes (e.g., walking, gardening, housework, golf, bowls, dancing, Tai Chi, swimming) 


\section{(self-management, continued)}

Check a box by crossing it:

Right now

\section{$\mathbf{x}$}

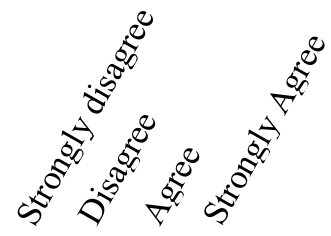

heiQ_10. I have plans to do enjoyable things for myself during the next few days.

heiQ_11. I have a very good understanding of when and why I am supposed to take my medication.

heiQ_12. I often feel angry when I think about my health.

heiQ_13. On most days of the week, I set aside time for healthy activities (e.g., walking, relaxation, exercise).

heiQ_14. I feel hopeless because of my health problems.

heiQ_15. I feel like I am actively involved in life.

heiQ_16. When I have health problems, I have a clear understanding of what I need to do to control them.

heiQ_17. I carefully watch my health and do what is necessary to keep as healthy as possible.

heiQ_18. I get upset when I think about my health.

heiQ_19. I walk for exercise, for at least 15 minutes per day, most days of the week.

heiQ_20. With my health in mind, I have realistic expectations of what I can and cannot do.

heiQ_21. If I think about my health, I get depressed.

heiQ_22. If I need help, I have plenty of people I can rely on. 


\section{(self-management, continued)}

Check a box by crossing it:

Right now

\section{$\mathbf{X}$}

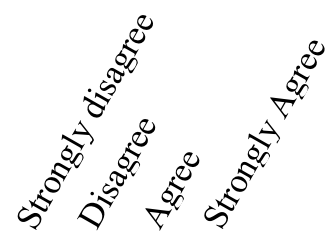

heiQ_23. I have effective ways to prevent my symptoms (e.g., discomfort, pain, and stress) from limiting what I can do in my life.

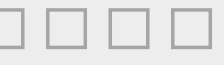

heiQ_24. I have very positive relationships with my healthcare professionals.

heiQ_25. I have a very good idea of how to manage my health problems.

heiQ_26. When I have symptoms, I have skills that help me cope.

heiQ_27. I try not to let my health problems stop me from enjoying life.

heiQ_28. I have enough friends who help me cope with my health problems.

heiQ_29. I communicate very confidently with my doctor about my healthcare needs.

heiQ_30. I have a good understanding of equipment that could make my life easier.

heiQ_31. When I feel ill, my family and carers really understand what I am going through.

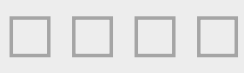

heiQ_32. I confidently give healthcare professionals the information they need to help.

heiQ_33. I get my needs met from available healthcare resources (e.g., doctors, hospitals, and community services).

heiQ_34. My health problems do not ruin my life. 


\section{(self-management, continued)}

Check a box by crossing it:

Right now

\section{$x$}

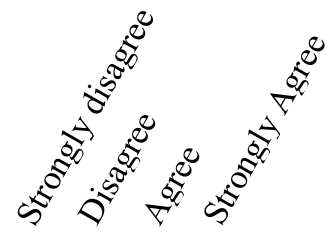

heiQ_35. Overall, I feel well looked after by friends or family.

heiQ_36. I feel I have a very good life even when I have health problems.

heiQ_37. I get enough chances to talk about my health problems with people who understand me.

heiQ_38. I work in a team with my doctors and other healthcare professionals.

heiQ_39. I do not let my health problems control my life.

heiQ_40. If others can cope with problems like mine, I can too. 


\section{Alcohol Use}

The following questions ask about your alcohol use and behaviors. Please recall that all answers will be kept confidential.

ETOH_1 In the past 30 days, did you drink any type of alcoholic beverages?

\begin{tabular}{cc} 
Yes $\quad$ No \\
\hline$\square$ & $\square$
\end{tabular}

If yes, proceed to items below (ETOH_2).

If no, skip the remaining items on this page and go to ETOH_7 on page 18.

\begin{tabular}{|c|c|c|c|c|c|c|}
\hline & In the past 30 days.. & $\begin{array}{c}1-2 \\
\text { drinks }\end{array}$ & $\begin{array}{c}\text { 3-4 } \\
\text { drinks }\end{array}$ & $\begin{array}{c}5-6 \\
\text { drinks }\end{array}$ & $\begin{array}{c}7-10 \\
\text { drinks }\end{array}$ & $\begin{array}{c}\text { More } \\
\text { than } \\
10 \\
\text { drinks }\end{array}$ \\
\hline$\overline{\mathrm{ETOH} \_2}$ & $\begin{array}{l}\text { On a typical day when I drank } \\
\text { alcohol, I had......................... }\end{array}$ & $\square$ & $\square$ & $\square$ & $\square$ & $\square$ \\
\hline ETOH_3 & $\begin{array}{l}\text { The largest number of drinks } \\
\text { that I had in a single day was..... }\end{array}$ & $\square$ & $\square$ & $\square$ & $\square$ & $\square$ \\
\hline & & Never & $\begin{array}{c}1-2 \\
\text { times }\end{array}$ & 3-5 times & $\begin{array}{c}6-10 \\
\text { times }\end{array}$ & $\begin{array}{c}\text { More } \\
\text { than } \\
10 \\
\text { times } \\
\end{array}$ \\
\hline 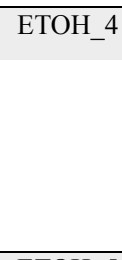 & I became drunk or intoxicated.... & Never & 1 time & 2 times & $\begin{array}{c}3 \\
\text { times } \\
\end{array}$ & $\begin{array}{l} \\
4 \text { or } \\
\text { more } \\
\text { times } \\
\end{array}$ \\
\hline$\overline{\text { ETOH_5 }}$ & $\begin{array}{l}\text { I spent a whole weekend } \\
\text { drinking..................... }\end{array}$ & $\square$ & $\square$ & $\square$ & $\square$ & $\square$ \\
\hline & & $\begin{array}{c}1-7 \\
\text { drinks }\end{array}$ & $\begin{array}{c}8-14 \\
\text { drinks }\end{array}$ & $\begin{array}{c}15-21 \\
\text { drinks }\end{array}$ & $\begin{array}{c}22-28 \\
\text { drinks }\end{array}$ & $\begin{array}{c}\text { More } \\
\text { than } \\
28 \\
\text { drinks } \\
\end{array}$ \\
\hline ETOH_6 & In a typical week I drank. & $\square$ & $\square$ & $\square$ & $\square$ & $\square$ \\
\hline
\end{tabular}




\section{Alcohol Use in Relation to Cancer Diagnosis and Treatment}

In the following section, you will be asked about when you were first told you had a second cancer. Please answer these questions about your second cancer diagnosis.

ETOH_7 I quit drinking alcohol more than 1

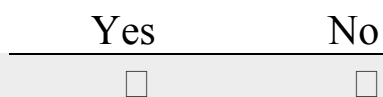
year before I was told I had a second cancer.

Also check "yes" if you never drank alcohol.

If yes, skip the remaining items on this page and go to CTUQ_1 on page 19.

If no, proceed to items below (ETOH_8).

ETOH_8. During each of the following time frames, please indicate whether you drank alcohol every day, some days, or not at all.

\begin{tabular}{|l|c|c|c|c|}
\hline & $\begin{array}{c}\text { Drank } \\
\text { every day }\end{array}$ & $\begin{array}{c}\text { Didn't } \\
\text { Dome days } \\
\text { some }\end{array}$ & $\begin{array}{c}\text { Don't } \\
\text { drink at } \\
\text { all }\end{array}$ & $\begin{array}{c}\text { Dnow/ Not } \\
\text { sure }\end{array}$ \\
\hline $\begin{array}{l}\text { ETOH_8a. The year before you were told } \\
\text { you had a second cancer }\end{array}$ & $\square$ & $\square$ & $\square$ & $\square$ \\
$\begin{array}{l}\text { ETOH_8b. After your second cancer } \\
\text { diagnosis, and before treatment for the } \\
\text { second cancer started }\end{array}$ & $\square$ & $\square$ & $\square$ & $\square$ \\
$\begin{array}{l}\text { ETOH_8c. During the course of } \\
\text { treatment for your second cancer }\end{array}$ & $\square$ & $\square$ & $\square$ & $\square$ \\
$\begin{array}{l}\text { ETOH_8d. After treatment ended for } \\
\text { your second cancer }\end{array}$ & $\square$ & $\square$ & $\square$ & $\square$ \\
\hline
\end{tabular}




\section{Tobacco Use}

Section 1. Basic Tobacco Use Information

CTUQ_1. Have you smoked at least 100 cigarettes ( 5 packs $=100$ cigarettes) in your entire life?

Yes

No $\rightarrow$ Go to PGLOB_1 (page 22).

Don't know / Not sure $\rightarrow$ Go to PGLOB_1 (page 22).

CTUQ_2. How old were you when you first smoked a cigarette (even one or two puffs)? years old

CTUQ_3. Have you ever smoked cigarettes regularly?

No. I have never smoked cigarettes regularly.

Yes

CTUQ_3a. If YES, how old were you when you first began smoking cigarettes regularly? years old

CTUQ_4. How many total years have you smoked (or did you smoke) cigarettes? Do not count any time you may have stayed off cigarettes.

Years If you smoked less than one year, write "1."

CTUQ_5. On average when you have smoked, about how many cigarettes do you (or did you) smoke a day?

A pack usually has 20 cigarettes in it.

Number of cigarettes per day 


\section{(tobacco use, continued)}

\section{CTUQ_6. How long has it been since you last smoked a cigarette (even one or two puffs)?}

First check which one of the following choices applies to you. Then, if applicable, write a number on the line for how many days, weeks, months, or years it has been since your last cigarette.

I smoked a cigarette today (at least one puff).

1-7 days. $\rightarrow$ CTUQ_6a. Number of days since last cigarette:

Less than 1 month. $\rightarrow$ CTUQ_6b. Number of weeks since last cigarette:

Less than 1 year. $\rightarrow$ CTUQ_6c. Number of months since last cigarette:

More than 1 year. $\rightarrow$ CTUQ_6d. Number of years since last cigarette:

Don't know / Don't remember 
(tobacco use, continued)

\section{INSTRUCTIONS}

In the following section, you will be asked about when you were first told you had a second cancer. Please answer these questions about your second cancer diagnosis.

Section 2. Tobacco Use in Relation to Cancer Diagnosis and Treatment

CTUQ_7 I quit smoking more than 1 year before

Yes No

I was told I had a second cancer.

Also check "yes" if you never smoked

or smoked less than 100 cigarettes in

your entire life.

If yes, skip the remaining items on this page and go to PGLOB_1 on page 22 .

If no, proceed to items below (CTUQ_8).

CTUQ_8. During each of the following time frames, please indicate whether you smoked cigarettes every day, some days, or not at all.

\begin{tabular}{|l|c|c|c|c|}
\hline & $\begin{array}{c}\text { Smoked } \\
\text { every day }\end{array}$ & $\begin{array}{c}\text { Smoked } \\
\text { some days }\end{array}$ & $\begin{array}{c}\text { Didn't } \\
\text { smoke at } \\
\text { all }\end{array}$ & $\begin{array}{c}\text { Don't } \\
\text { know/ Not } \\
\text { sure }\end{array}$ \\
\hline $\begin{array}{l}\text { CTUQ_8a. The year before you were } \\
\text { told you had a second cancer }\end{array}$ & $\square$ & $\square$ & $\square$ & $\square$ \\
\hline $\begin{array}{l}\text { CTUQ_8b. After your second cancer } \\
\text { diagnosis, and before treatment for } \\
\text { the second cancer started }\end{array}$ & $\square$ & $\square$ & $\square$ & \\
\hline $\begin{array}{l}\text { CTUQ_8c. During the course of } \\
\text { treatment for your second cancer }\end{array}$ & $\square$ & $\square$ & $\square$ & $\square$ \\
\hline $\begin{array}{l}\text { CTUQ_8d. After treatment ended for } \\
\text { your second cancer }\end{array}$ & $\square$ & $\square$ & $\square$ & $\square$ \\
\hline
\end{tabular}




\section{Overall Health}

Please respond to each item by marking one box per row.

\begin{tabular}{|c|c|c|c|c|c|c|}
\hline & & $\begin{array}{c}\text { Excellent } \\
5 \\
\end{array}$ & $\begin{array}{c}\text { Very } \\
\text { Good } \\
4 \\
\end{array}$ & $\begin{array}{c}\text { Good } \\
\mathbf{3} \\
\end{array}$ & $\begin{array}{c}\text { Fair } \\
2\end{array}$ & $\begin{array}{c}\text { Poor } \\
1 \\
\end{array}$ \\
\hline PGLOB_1 & $\begin{array}{l}\text { In general, would you say } \\
\text { your health is: ............. }\end{array}$ & $\square$ & $\square$ & $\square$ & $\square$ & $\square$ \\
\hline PGLOB_2 & $\begin{array}{l}\text { In general, would you say } \\
\text { your quality of life is: ...... }\end{array}$ & $\square$ & $\square$ & $\square$ & $\square$ & $\square$ \\
\hline PGLOB_3 & $\begin{array}{l}\text { In general, how would you } \\
\text { rate your physical health? ... }\end{array}$ & $\square$ & $\square$ & $\square$ & $\square$ & $\square$ \\
\hline PGLOB_4 & $\begin{array}{l}\text { In general, how would you } \\
\text { rate your mental health, } \\
\text { including your mood and } \\
\text { your ability to think? ......... }\end{array}$ & $\square$ & $\square$ & $\square$ & $\square$ & $\square$ \\
\hline PGLOB_5 & $\begin{array}{l}\text { In general, how would you } \\
\text { rate your satisfaction with } \\
\text { your social activities and } \\
\text { relationships? ............... }\end{array}$ & $\square$ & $\square$ & $\square$ & $\square$ & $\square$ \\
\hline PGLOB_6 & $\begin{array}{l}\text { In general, please rate how } \\
\text { well you carry out your usual } \\
\text { social activities and roles. } \\
\text { (This includes activities at } \\
\text { home, at work and in your } \\
\text { community, and } \\
\text { responsibilities as a parent, } \\
\text { child, spouse, employee, } \\
\text { friend, etc.).................... }\end{array}$ & $\square$ & $\square$ & $\square$ & $\square$ & $\square$ \\
\hline
\end{tabular}




\section{(overall health, continued)}

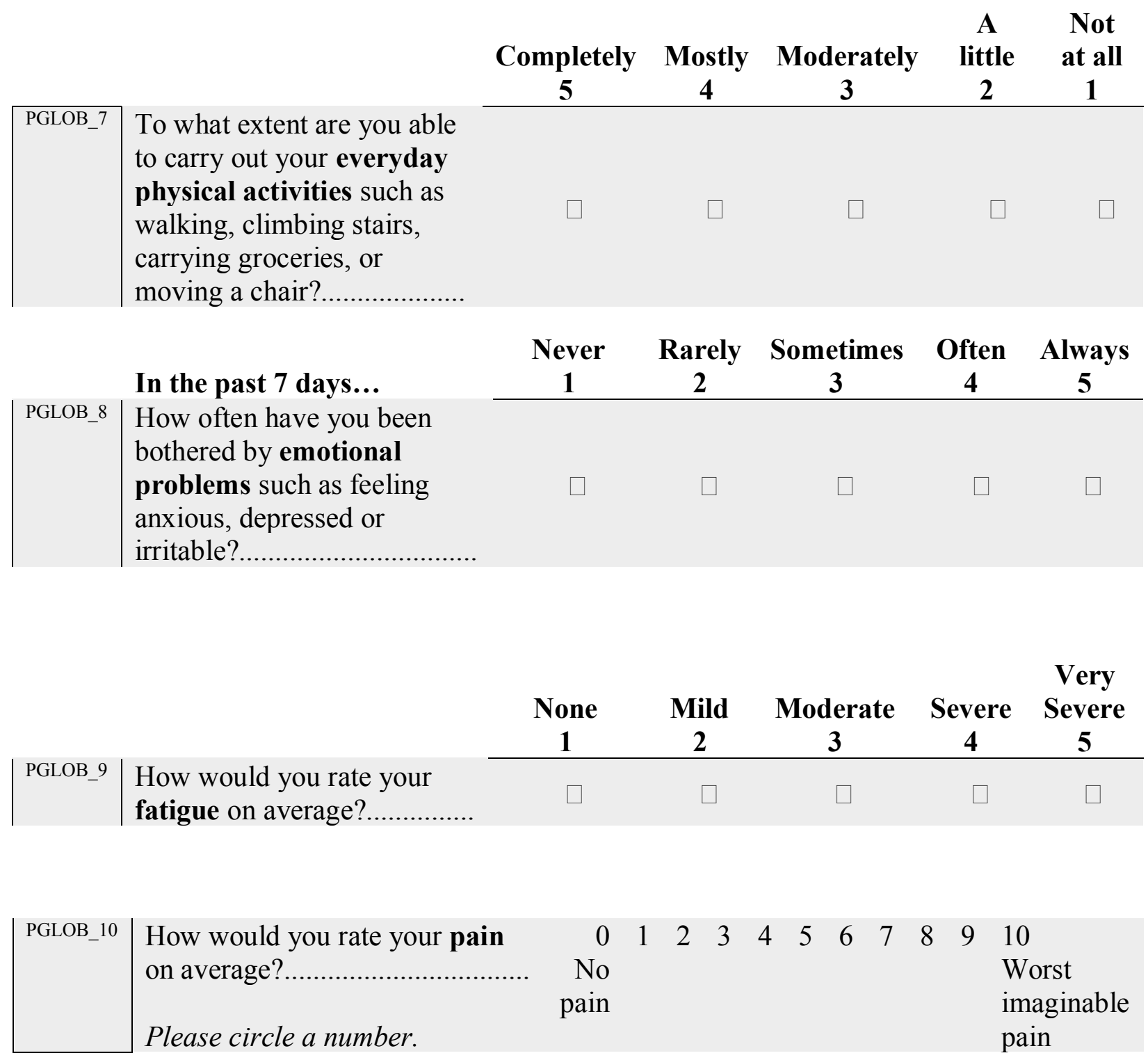




\section{Physical Function}

Please respond to each item by marking one box per row.

\begin{tabular}{|c|c|c|c|c|c|c|}
\hline & & $\begin{array}{c}\text { Not at all } \\
5\end{array}$ & $\begin{array}{c}\text { Very } \\
\text { little } \\
4\end{array}$ & $\begin{array}{c}\text { Somewhat } \\
3 \\
\end{array}$ & $\begin{array}{l}\text { Quite } \\
\text { a lot } \\
2\end{array}$ & $\begin{array}{c}\text { Cannot } \\
\text { do } \\
1\end{array}$ \\
\hline PFX_1 & $\begin{array}{l}\text { Does your health now limit you in } \\
\text { doing vigorous activities, such as } \\
\text { running, lifting heavy objects, } \\
\text { participating in strenuous sports?...... }\end{array}$ & $\square$ & $\square$ & $\square$ & $\square$ & $\square$ \\
\hline PFX_2 & $\begin{array}{l}\text { Does your health now limit you in } \\
\text { walking more than a mile?............... }\end{array}$ & $\square$ & $\square$ & $\square$ & $\square$ & $\square$ \\
\hline PFX_3 & $\begin{array}{l}\text { Does your health now limit you in } \\
\text { climbing one flight of stairs?............ }\end{array}$ & $\square$ & $\square$ & $\square$ & $\square$ & $\square$ \\
\hline PFX_4 & $\begin{array}{l}\text { Does your health now limit you in } \\
\text { lifting or carrying groceries?............ }\end{array}$ & $\square$ & $\square$ & $\square$ & $\square$ & $\square$ \\
\hline PFX_5 & $\begin{array}{l}\text { Does your health now limit you in } \\
\text { bending, kneeling, or stooping?........ }\end{array}$ & $\square$ & $\square$ & $\square$ & $\square$ & $\square$ \\
\hline
\end{tabular}

\begin{tabular}{|c|c|}
\hline PFX_6 & $\begin{array}{l}\text { Are you able to do chores such as } \\
\text { vacuuming or yard work?.............. }\end{array}$ \\
\hline PFX_7 & $\begin{array}{l}\text { Are you able to dress yourself, } \\
\text { including tying shoelaces and } \\
\text { doing buttons?............................. }\end{array}$ \\
\hline PFX_8 & $\begin{array}{l}\text { Are you able to shampoo your } \\
\text { hair? }\end{array}$ \\
\hline PFX_9 & 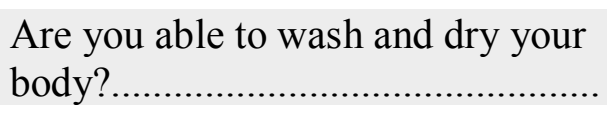 \\
\hline PFX_10 & $\begin{array}{l}\text { Are you able to get on and off the } \\
\text { toilet? }\end{array}$ \\
\hline
\end{tabular}

\begin{tabular}{|c|c|c|c|c|}
\hline $\begin{array}{c}\text { Without } \\
\text { any } \\
\text { difficulty } \\
5\end{array}$ & $\begin{array}{c}\text { With a } \\
\text { little } \\
\text { difficulty } \\
4\end{array}$ & $\begin{array}{c}\text { With } \\
\text { some } \\
\text { difficulty } \\
3\end{array}$ & $\begin{array}{c}\text { With } \\
\text { much } \\
\text { difficulty } \\
2\end{array}$ & $\begin{array}{c}\text { Unable } \\
\text { to do } \\
1\end{array}$ \\
\hline
\end{tabular}




\section{Symptoms}

Part I. How severe are your symptoms?

People with cancer frequently have symptoms that are caused by their disease or by their treatment. We ask you to rate how severe the following symptoms have been in the last $\underline{\mathbf{2 4}}$ hours. Please select a number from 0 (symptom has not been present) to 10 (the symptom was as bad as you can imagine it could be) for each item.

\begin{tabular}{|c|c|c|c|c|c|c|c|c|c|c|c|}
\hline \multirow{2}{*}{$\begin{array}{l}\text { Please circle one number per row. } \\
\text { MDASI_1. Your pain at its WORST? }\end{array}$} & \multicolumn{8}{|c|}{$\begin{array}{l}\text { Not } \\
\text { Present }\end{array}$} & \multicolumn{3}{|c|}{$\begin{array}{l}\text { As Bad As } \\
\text { You } \\
\text { Can Imagin }\end{array}$} \\
\hline & 0 & 1 & 2 & 3 & 4 & 5 & 6 & 7 & 8 & 9 & 10 \\
\hline $\begin{array}{l}\text { MDASI_2. Your fatigue (tiredness) at its } \\
\text { WORST? }\end{array}$ & 0 & 1 & 2 & 3 & 4 & 5 & 6 & 7 & 8 & 9 & 10 \\
\hline MDASI_3. Your nausea at its WORST? & 0 & 1 & 2 & 3 & 4 & 5 & 6 & 7 & 8 & 9 & 10 \\
\hline $\begin{array}{l}\text { MDASI_4. Your disturbed sleep at its } \\
\text { WORST? }\end{array}$ & 0 & 1 & 2 & 3 & 4 & 5 & 6 & 7 & 8 & 9 & 10 \\
\hline $\begin{array}{l}\text { MDASI_5. Your feelings of being distressed } \\
\text { (upset) at its WORST? }\end{array}$ & 0 & 1 & 2 & 3 & 4 & 5 & 6 & 7 & 8 & 9 & 10 \\
\hline $\begin{array}{l}\text { MDASI_6. Your shortness of breath at its } \\
\text { WORST? }\end{array}$ & 0 & 1 & 2 & 3 & 4 & 5 & 6 & 7 & 8 & 9 & 10 \\
\hline $\begin{array}{l}\text { MDASI_7. Your problem with remembering } \\
\text { things at its WORST? }\end{array}$ & 0 & 1 & 2 & 3 & 4 & 5 & 6 & 7 & 8 & 9 & 10 \\
\hline $\begin{array}{l}\text { MDASI_8. Your problem with lack of } \\
\text { appetite at its WORST? }\end{array}$ & 0 & 1 & 2 & 3 & 4 & 5 & 6 & 7 & 8 & 9 & 10 \\
\hline $\begin{array}{l}\text { MDASI_9. Your feeling drowsy (sleepy) at } \\
\text { its WORST? }\end{array}$ & 0 & 1 & 2 & 3 & 4 & 5 & 6 & 7 & 8 & 9 & 10 \\
\hline $\begin{array}{l}\text { MDASI_10. Your having a dry mouth at its } \\
\text { WORST? }\end{array}$ & 0 & 1 & 2 & 3 & 4 & 5 & 6 & 7 & 8 & 9 & 10 \\
\hline
\end{tabular}




\section{(symptoms, continued)}

\begin{tabular}{|c|c|c|c|c|c|c|c|c|c|c|c|}
\hline Please circle one number per row. & \multicolumn{8}{|c|}{$\begin{array}{l}\text { Not } \\
\text { Present }\end{array}$} & \multicolumn{3}{|c|}{$\begin{array}{l}\text { As Bad As } \\
\text { You } \\
\text { Can Imagine }\end{array}$} \\
\hline $\begin{array}{l}\text { MDASI_11. Your feeling sad at its } \\
\text { WORST? }\end{array}$ & 0 & 1 & 2 & 3 & 4 & 5 & 6 & 7 & 8 & 9 & 10 \\
\hline MDASI_12. Your vomiting at its WORST? & 0 & 1 & 2 & 3 & 4 & 5 & 6 & 7 & 8 & 9 & 10 \\
\hline $\begin{array}{l}\text { MDASI_13. Your numbness or tingling at } \\
\text { its WORST? }\end{array}$ & 0 & 1 & 2 & 3 & 4 & 5 & 6 & 7 & 8 & 9 & 10 \\
\hline
\end{tabular}

Part II. How have your symptoms interfered with your life?

Symptoms frequently interfere with how we feel and function. How much have your symptoms interfered with the following items in the last 24 hours? Please select a number from 0 (symptoms have not interfered) to 10 (symptoms interfered completely) for each item.

\begin{tabular}{|c|c|c|c|c|c|c|c|c|c|c|c|}
\hline Please circle one number per row. & $\begin{array}{l}\text { Did } \\
\text { Inte }\end{array}$ & & & & & & & & & \multicolumn{2}{|c|}{$\begin{array}{l}\text { Interfered } \\
\text { Completely }\end{array}$} \\
\hline MDASI_14. General activity? & 0 & 1 & 2 & 3 & 4 & 5 & 6 & 7 & 8 & 9 & 10 \\
\hline MDASI_15. Mood? & 0 & 1 & 2 & 3 & 4 & 5 & 6 & 7 & 8 & 9 & 10 \\
\hline $\begin{array}{l}\text { MDASI_16. Work (including } \\
\text { work around the } \\
\text { house)? }\end{array}$ & 0 & 1 & 2 & 3 & 4 & 5 & 6 & 7 & 8 & 9 & 10 \\
\hline $\begin{array}{l}\text { MDASI_17. Relations with other } \\
\text { people? }\end{array}$ & 0 & 1 & 2 & 3 & 4 & 5 & 6 & 7 & 8 & 9 & 10 \\
\hline MDASI_18. Walking? & 0 & 1 & 2 & 3 & 4 & 5 & 6 & 7 & 8 & 9 & 10 \\
\hline MDASI_19. Enjoyment of life? & 0 & 1 & 2 & 3 & 4 & 5 & 6 & 7 & 8 & 9 & 10 \\
\hline
\end{tabular}




\section{Health History}

Please use this form to indicate with a check whether you have any of the following health conditions you may have experienced or be dealing with presently.

Please check yes or no for each row.

CCI_1. Asthma, emphysema, or chronic bronchitis

CCI_2. Arthritis or rheumatism

CCI_3. Cancer diagnosed in the past $\underline{3 \text { years }}$

CCI_4. Diabetes

CCI_5. Digestive problems (such as ulcer, colitis, or gallbladder disease)

CCI_6. Heart trouble (such as angina, congestive heart failure, or coronary artery disease)

CCI_7. HIV illness or AIDS

CCI_8. Kidney disease

CCI_9. Liver problems (such as cirrhosis)

CCI_10. Stroke 


\section{Financial Impact of Cancer}

People who have experienced cancer may be impacted financially. We are trying to learn the financial impacts of having multiple cancers.

The next set of questions will ask you about financial problems you may have experienced. As with the rest of the study, all responses will be kept confidential.

Below is a list of statements that other people with cancer have said are important. Please check one box per line to indicate your response as it applies to the past 7 days.

\begin{tabular}{|c|c|c|c|c|c|c|}
\hline & & $\begin{array}{l}\text { Not } \\
\text { at all } \\
0\end{array}$ & $\begin{array}{l}\text { A } \\
\text { little } \\
\text { bit } \\
1\end{array}$ & $\begin{array}{l}\text { Some- } \\
\text { what } \\
2\end{array}$ & $\begin{array}{l}\text { Quite } \\
\text { a bit } \\
\quad 3\end{array}$ & $\begin{array}{l}\text { Very } \\
\text { much } \\
\quad 4\end{array}$ \\
\hline COST_1 & 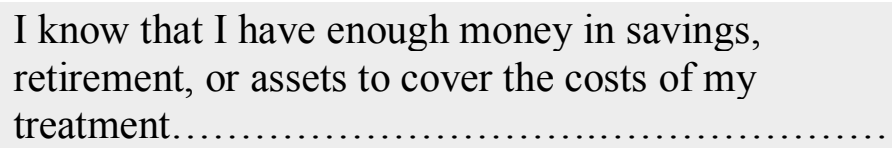 & $\square$ & $\square$ & $\square$ & $\square$ & $\square$ \\
\hline COST_2 & 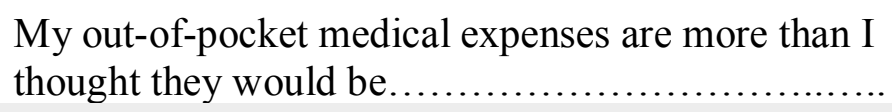 & $\square$ & $\square$ & $\square$ & $\square$ & $\square$ \\
\hline COST_3 & $\begin{array}{l}\text { I worry about the financial problems I will have in } \\
\text { the future as a result of my illness or treatment....... }\end{array}$ & $\square$ & $\square$ & $\square$ & $\square$ & $\square$ \\
\hline COST_4 & 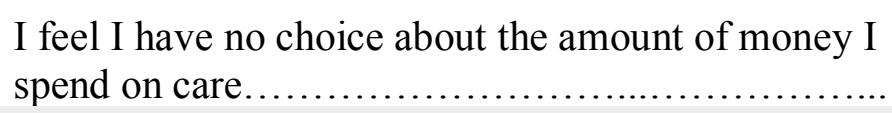 & $\square$ & $\square$ & $\square$ & $\square$ & $\square$ \\
\hline COST_5 & 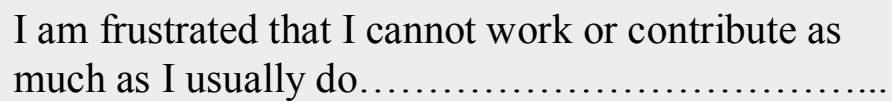 & $\square$ & $\square$ & $\square$ & $\square$ & $\square$ \\
\hline COST_6 & I am satisfied with my current financial situation..... & $\square$ & $\square$ & $\square$ & $\square$ & $\square$ \\
\hline COST_7 & I am able to meet my monthly expenses.............. & $\square$ & $\square$ & $\square$ & $\square$ & $\square$ \\
\hline COST_8 & I feel financially stressed $\ldots \ldots \ldots \ldots \ldots \ldots \ldots \ldots \ldots$ & $\square$ & $\square$ & $\square$ & $\square$ & $\square$ \\
\hline COST_9 & 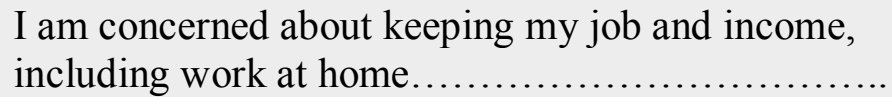 & $\square$ & $\square$ & $\square$ & $\square$ & $\square$ \\
\hline COST_10 & $\begin{array}{l}\text { My cancer or treatment has reduced my satisfaction } \\
\text { with my present financial situation.................. }\end{array}$ & $\square$ & $\square$ & $\square$ & $\square$ & $\square$ \\
\hline COST_11 & I feel in control of my financial situation.............. & $\square$ & $\square$ & $\square$ & $\square$ & 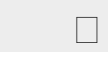 \\
\hline
\end{tabular}




\section{Financial Hardships}

Please check one answer per row that best applies to you.

$\underline{\text { Financial Strain }}$

EH_1. In the next three months, how often do you think that you and your family will experience bad times such as poor housing or not having enough food?

\begin{tabular}{ccccc}
$\begin{array}{c}\text { Almost } \\
\text { never }\end{array}$ & $\begin{array}{c}\text { Once in a } \\
\text { while }\end{array}$ & $\begin{array}{c}\text { A lot of the } \\
\text { time }\end{array}$ & $\begin{array}{c}\text { Almost } \\
\text { Sometimes }\end{array}$ & $\begin{array}{c}\text { (frequently) } \\
\text { always }\end{array}$ \\
\hline & 2 & 3 & 4 & 5
\end{tabular}

EH_2. In the next three months, how often do you expect that you will have to do without the basic things that your family needs?

\section{Inability to Make Ends Meet}

EH_3. Think back over the past 3 months and tell us how much difficulty you had paying your bills. Would you say you had...

EH_4. Think again over the past 3

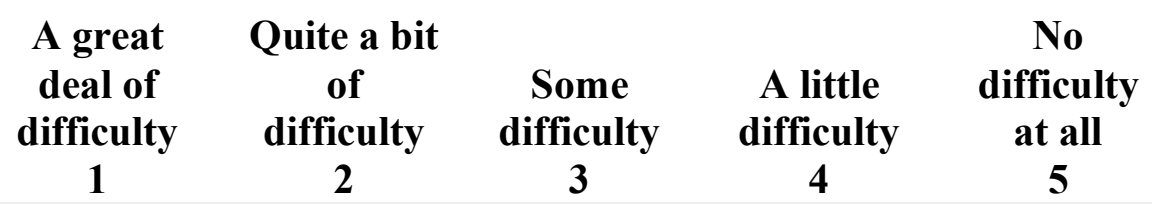
months. Generally, at the end of each month, did you end up with...

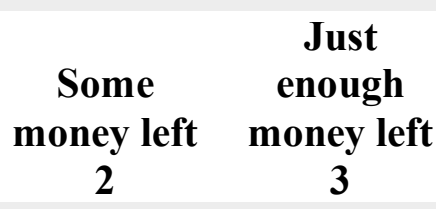

\section{Very short of money 5}




\section{(financial hardships, continued)}

Please check one answer per row that best applies to you.

\section{Not Enough Money for Necessities}

Please think about how you felt about your family's economic situation over the past 3 months. Indicate how much you would agree or disagree with each statement.

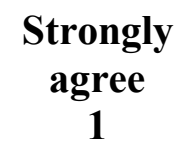

EH_5. My family had enough money to afford the kind of home we should have.

EH_6. We had enough money to afford the kind of clothing we should have.

EH_7. We had enough money to afford the kind of furniture or household appliances we should have.

EH_8. We had enough money to afford the kind of car we need.

EH_9. We had enough money to afford the kind of food we should have.

EH_10. We had enough money to afford the kind of medical care we should have.

EH_11. My family had enough money to afford leisure and recreational activities.

$\begin{array}{cc}\text { Agree } & \begin{array}{c}\text { Neutral/ } \\ \text { Mixed }\end{array} \\ \mathbf{2} & \mathbf{3}\end{array}$

Disagree

Strongly

disagree

5 


\section{(financial hardships, continued)}

Please check one answer per row that best applies to you.

\section{Economic Adjustments/Cutbacks}

In the past 3 months, has your family made any of the following adjustments because of financial need?

$\begin{array}{cc}\text { Yes } & \text { No } \\ 1 & 2\end{array}$

EH_12. Changed food shopping or eating habits a lot to save money

EH_13. Shut down the heat or air conditioning to save money even though it made the house uncomfortable

EH_14. Didn't go to see the doctor or dentist when you needed to because you had to save money

EH_15. Fell far behind in paying bills

EH_16. Asked relatives or friends for money or food to help you get by

EH_17. Added another job to help make ends meet

EH_18. Received government assistance

EH_19. Sold some possessions because you needed the money (even though you really wanted to keep them)

EH_20. Moved to another house or apartment to save money 


\section{Ability to Participate in Social Roles and Activities}

Please respond to each item by marking one box per row.

\begin{tabular}{|c|c|c|c|c|c|c|}
\hline & & $\begin{array}{c}\text { Never } \\
5\end{array}$ & $\begin{array}{c}\text { Rarely } \\
4\end{array}$ & $\begin{array}{c}\text { Some- } \\
\text { times } \\
3\end{array}$ & $\begin{array}{c}\text { Usually } \\
2 \\
\end{array}$ & $\begin{array}{c}\text { Always } \\
1 \\
\end{array}$ \\
\hline PABIL_1 & 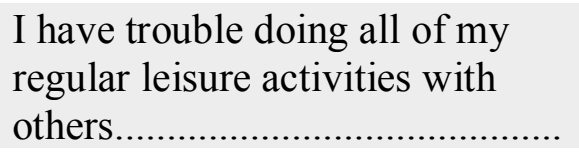 & $\square$ & $\square$ & $\square$ & $\square$ & $\square$ \\
\hline PABIL_2 & 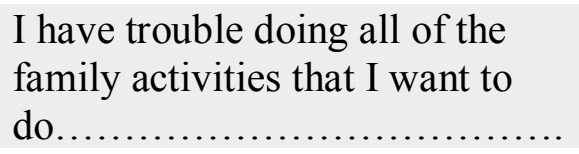 & $\square$ & $\square$ & $\square$ & $\square$ & $\square$ \\
\hline PABIL_3 & $\begin{array}{l}\text { I have trouble doing all of my } \\
\text { usual work (include work at } \\
\text { home)........................ }\end{array}$ & $\square$ & $\square$ & $\square$ & $\square$ & $\square$ \\
\hline PABIL_4 & $\begin{array}{l}\text { I have trouble doing all of the } \\
\text { activities with friends that I want } \\
\text { to do........................... }\end{array}$ & $\square$ & $\square$ & $\square$ & $\square$ & $\square$ \\
\hline PABIL_5 & $\begin{array}{l}\text { I have to limit the things I do for } \\
\text { fun with others................. }\end{array}$ & $\square$ & $\square$ & $\square$ & $\square$ & $\square$ \\
\hline PABIL_6 & $\begin{array}{l}\text { I have to limit my regular } \\
\text { activities with friends............ }\end{array}$ & $\square$ & $\square$ & $\square$ & $\square$ & $\square$ \\
\hline PABIL_7 & $\begin{array}{l}\text { I have to limit my regular family } \\
\text { activities....................... }\end{array}$ & $\square$ & $\square$ & $\square$ & $\square$ & $\square$ \\
\hline PABIL_8 & $\begin{array}{l}\text { I have trouble doing all of the } \\
\text { work that is really important to } \\
\text { me (including work at home)..... }\end{array}$ & $\square$ & $\square$ & $\square$ & $\square$ & $\square$ \\
\hline
\end{tabular}




\section{$\underline{\text { Satisfaction with Participation in Social Roles }}$}

Please respond to each question or statement by marking one box per row.

\begin{tabular}{|c|c|c|c|c|c|c|}
\hline & In the past 7 days... & $\begin{array}{l}\text { Not at } \\
\text { all } \\
5\end{array}$ & $\begin{array}{c}\text { A little } \\
\text { bit } \\
4 \\
\end{array}$ & $\begin{array}{c}\text { Some- } \\
\text { what } \\
3\end{array}$ & $\begin{array}{l}\text { Quite a } \\
\text { bit } \\
2\end{array}$ & $\begin{array}{c}\text { Very } \\
\text { much } \\
1 \\
\end{array}$ \\
\hline PSAT_1 & $\begin{array}{l}\text { I am satisfied with how much } \\
\text { work I can do (include work at } \\
\text { home) } \ldots \ldots \ldots \ldots \ldots \ldots \ldots \ldots \ldots \ldots \ldots \ldots\end{array}$ & $\square$ & $\square$ & $\square$ & $\square$ & $\square$ \\
\hline PSAT_2 & $\begin{array}{l}\text { I am satisfied with my ability to } \\
\text { work (include work at home)..... }\end{array}$ & $\square$ & $\square$ & $\square$ & $\square$ & $\square$ \\
\hline PSAT_3 & $\begin{array}{l}\text { I am satisfied with my ability to } \\
\text { do regular personal and } \\
\text { household responsibilities........ }\end{array}$ & $\square$ & $\square$ & $\square$ & $\square$ & $\square$ \\
\hline PSAT_4 & $\begin{array}{l}\text { I am satisfied with my ability to } \\
\text { perform my daily routines......... }\end{array}$ & $\square$ & $\square$ & $\square$ & $\square$ & $\square$ \\
\hline PSAT_5 & $\begin{array}{l}\text { I am satisfied with my ability to } \\
\text { meet the needs of those who } \\
\text { depend on me................... }\end{array}$ & $\square$ & $\square$ & $\square$ & $\square$ & $\square$ \\
\hline PSAT_6 & $\begin{array}{l}\text { I am satisfied with my ability to } \\
\text { do household chores/tasks......... }\end{array}$ & $\square$ & $\square$ & $\square$ & $\square$ & $\square$ \\
\hline PSAT_7 & $\begin{array}{l}\text { I am satisfied with my ability to } \\
\text { do things for my family............ }\end{array}$ & $\square$ & $\square$ & $\square$ & $\square$ & $\square$ \\
\hline PSAT_8 & $\begin{array}{l}\text { I am satisfied with the amount of } \\
\text { time I spend performing my } \\
\text { daily routines..................... }\end{array}$ & $\square$ & $\square$ & $\square$ & $\square$ & $\square$ \\
\hline
\end{tabular}




\section{General Outlook}

Please use the rating scale below to indicate how accurately each statement describes you.

How much do you agree with each statement about you as you generally are now, not as you wish to be in the future?

\begin{tabular}{|c|c|c|c|c|c|}
\hline In general, I... & $\begin{array}{c}\text { Strongly } \\
\text { Disagree } \\
1 \\
\end{array}$ & $\begin{array}{c}\text { Somewhat } \\
\text { Agree } \\
2 \\
\end{array}$ & $\begin{array}{c}\text { Neither } \\
\text { Agree nor } \\
\text { Disagree } \\
\mathbf{3} \\
\end{array}$ & $\begin{array}{c}\text { Somewhat } \\
\text { Agree } \\
4\end{array}$ & $\begin{array}{c}\text { Strongly } \\
\text { Agree } \\
5 \\
\end{array}$ \\
\hline IPIP_1. Get chores done right away. & $\square$ & $\square$ & $\square$ & $\square$ & $\square$ \\
\hline IPIP_2. Have frequent mood swings. & $\square$ & $\square$ & $\square$ & $\square$ & $\square$ \\
\hline $\begin{array}{l}\text { IPIP_3. Often forget to put things back in } \\
\text { their proper place. }\end{array}$ & $\square$ & $\square$ & $\square$ & $\square$ & $\square$ \\
\hline IPIP_4. Am relaxed most of the time. & $\square$ & $\square$ & $\square$ & $\square$ & $\square$ \\
\hline IPIP_5. Like order. & $\square$ & $\square$ & $\square$ & $\square$ & $\square$ \\
\hline IPIP_6. Get upset easily. & $\square$ & $\square$ & $\square$ & $\square$ & $\square$ \\
\hline IPIP_7. Make a mess of things. & $\square$ & $\square$ & $\square$ & $\square$ & $\square$ \\
\hline IPIP_8. Seldom feel blue. & $\square$ & $\square$ & $\square$ & $\square$ & $\square$ \\
\hline
\end{tabular}




\section{Social Support}

Instructions: This scale is made up of a list of statements each of which may or may not be true about you. For each statement circle "definitely true" if you are sure it is true about you and "probably true" if you think it is true but are not absolutely certain. Similarly, you should circle "definitely false" if you are sure the statement is false and "probably false" if you think it is false but are not absolutely certain.

ISEL_1. If I wanted to go on a trip for a day (for example, to the country or mountains), I would have a hard time finding someone to go with me.
1. definitely false
2. probably false
3. probably true
4. definitely true

ISEL_2. I feel that there is no one I can share my most private worries and fears with.
1. definitely false
2. probably false
3. probably true
4. definitely true

ISEL_3. If I were sick, I could easily find someone to help me with my daily chores.
1. definitely false
2. probably false
3. probably true
4. definitely true

ISEL_4. There is someone I can turn to for advice about handling problems with my family.
1. definitely false
2. probably false
3. probably true
4. definitely true

ISEL_5. If I decide one afternoon that I would like to go to a movie that evening, I could easily find someone to go with me.
1. definitely false
2. probably false
3. probably true
4. definitely true

ISEL_6. When I need suggestions on how to deal with a personal problem, I know someone I can turn to.
1. definitely false
2. probably false
3. probably true
4. definitely true

ISEL_7. I don't often get invited to do things with others.
1. definitely false
2. probably false
3. probably true
4. definitely true

ISEL_8. If I had to go out of town for a few weeks, it would be difficult to find someone who would look after my house or apartment (the plants, pets, garden, etc.).
1. definitely false
2. probably false
3. probably true
4. definitely true

ISEL_9. If I wanted to have lunch with someone, I could easily find someone to join me.
1. definitely false
2. probably false
3. probably true
4. definitely true

ISEL_10. If I was stranded 10 miles from home, there is someone I could call who could come and get me.
1. definitely false
2. probably false
3. probably true
4. definitely true

ISEL11. If a family crisis arose, it would be difficult to find someone who could give me good advice about how to handle it.
1. definitely false
2. probably false
3. probably true
4. definitely true

ISEL_12. If I needed some help in moving to a new house or apartment, I would have a hard time finding someone to help me.
1. definitely false
2. probably false
3. probably true
4. definitely true 


\section{Socio-demographic Questionnaire - Part 2}

SD_13. Which of the following best describes your current marital status?

- Never married

- Currently married

- Living with partner / significant other

- Widowed

- Separated

- Divorced

- Other (SD_13a. specify )

SD_14. How many years have you been at your current marital status? Answer " 1 " if less than one year.

SD_15. Is English your primary language (the one you speak most often)? $\bullet$ Yes $\bullet$ No

SD_16. What is the zip code where you live most of the time? (5-digit)

SD_17. In what type of area did you live most of your childhood?

- Urban, large city

- Urban, small city

- Suburb of large city

- Suburb of small city

- Rural, farm

- Rural, non-farm

- Other (SD_17a. specify ) 


\section{(socio-demographics, continued)}

SD_18. How many total years of formal education do you have?

SD_19. What is the highest grade or degree you have completed?

- Grade school (grades 1-8)

- High school diploma (grades 9-12)

- GED (Graduate Equivalent Degree)

- Vocational/Technical school certificate

- 2-year college (Associate’s level)

- 4-year college (Bachelor's level)

$\square$ Graduate school (Master's level)

- Professional school (i.e., MD, DVM, JD)

- Graduate school (Doctoral level, i.e., PhD, EdD)

- Unknown

SD_20. What is your current employment status?

- Full time (working at least 35 hours a week)

- Part time (working less than 35 hours a week)

- Laid off or unemployed, looking for work

- Laid off or unemployed, not looking for work

- Retired, not working at all

- Retired, but working part or full time

- Disabled/unable to work

- Full time homemaker

- Student

- Other (SD_20a. specify ___ 


\section{(socio-demographics, continued)}

SD_21. Are you currently employed? $\quad$ Y Yes • No

If you are NOT currently employed:

SD_21a. What was your primary occupation?

SD_21b. When was the last year you were employed?

If you ARE currently employed:

SD_21c. What is your primary occupation?

SD_22. Did you make changes at work since your second cancer diagnosis?

- Yes - No (If no, skip to SD_23 on page 39.)

SD_22a. If YES, why?

- I changed because of the physical demands of my job.

- I changed because of the mental demands of my job.

- I changed for other reasons.

- No, my change in occupation was not because of my cancer.

SD_22b. If YES, what change(s) did you make at work since your second cancer diagnosis?

Please specify:

SD_22c. Did that change affect your insurance coverage? $\quad$ Yes $・$ No SD_22d. If YES, please specify how:

SD_22e. Did that change affect your retirement benefits? - Yes - No SD_22f. If YES, please specify how: 


\section{(socio-demographics, continued)}

SD_23. Has your cancer or treatment caused you to take paid time off work? $\quad$ Yes $\bullet$ No SD_23a. If YES, how many days in the past month? Please specify:

SD_24. Has your cancer or treatment caused to take unpaid time off work? SD_24a. If YES, how many days in the past month? Please specify:

SD_25. Do you have any children? - Yes - No (If no, skip to SD_26.)

SD_25a. If YES, specify number of children:

SD_25b. If YES, specify current age(s) of children:

For example, you might write, " $2,7,21$ " on the line below, if these are the ages of your children.

SD_26. How many people presently live in your household, including yourself?

SD_26a. Adults:

SD_26b. Children (under 18 years old): 


\section{(socio-demographics, continued)}

SD_27. How important is religion or spirituality in your life?

- Not at all important

- Somewhat important

- Extremely important

SD_28. Do you have a religious background or preference? - Yes - No (If no, skip to SD_29 on page 41.)

SD_28a. If $\underline{\text { YES}}$, please specify:

- Christianity

- Judaism

- Islam

- Hinduism

- Buddhism

- Other (SD_28b. specify:

SD_28c. To what extent do you follow the customs and practices of your religion?

- Never

- Sometimes

- Frequently

- Always 


\section{(socio-demographics, continued)}

SD_29. Do you have health care insurance? - Yes - No (If no, skip to SD_30, page 42.)

SD_29a. If YES, please specify type:

- Medicare

- Medicaid/Medical Assistance

- SSI

- Veterans Administration

- Workers Compensation

- Private health insurance (SD_29b. specify

- Other (SD_29c. specify )

SD_29d. Does your insurance cover the cost of your medications?

- Yes, all the cost

- Yes, some of the cost (SD_29e. specify

- No

- Unknown

SD_29f. Does your insurance cover the cost of your health care?

- Yes, all the cost

- Yes, some of the cost - for example, it does not cover the cost of co-pays or other expenses (SD_29g. specify

- No

- Unknown 


\section{(socio-demographics, continued)}

SD_30. What are the sources of your own total gross annual income (all sources of income before taxes)? Select all that apply:

- Wages, salaries, commissions, bonus, tips from all jobs

- Self employment income from farm or non-farm business

- Interest, dividend, net rental income, royalties, or from estates or trusts

- Social security or railroad retirement

- Supplemental security income or other public assistance income

- Retirement, survivor, or disability pensions

- Other (SD_30a. please specify )

SD_31. What is the total gross annual income for your household (all sources of income before taxes and donations)?

- Under $\$ 10,000$

- $\$ 10,000-\$ 14,999$

- $\$ 15,000-\$ 19,999$

- $\$ 20,000-\$ 29,999$

- $\$ 30,000-\$ 39,999$

- $\$ 40,000-\$ 49,999$

- $\$ 50,000-\$ 59,999$

- $\$ 60,000-\$ 69,999$

- $\$ 70,000-\$ 79,999$

- $\$ 80,000$ - $\$ 99,999$

- $\$ 100,000-\$ 150,000$

- Over $\$ 150,000$

- Unknown

- Refuse/Prefer not to answer 
SD32. How difficult is it to pay for your basic needs?

- Not at all difficult

- Somewhat difficult

- Extremely difficult 


\section{Health Care Use}

HCU_1. In the past 6 months, how many times did you visit a physician? Do NOT include visits while in the hospital or the hospital emergency room. visits

HCU_2. In the past 6 months, how many times did you go to a hospital emergency room? times

HCU_3. How many different times did you stay in a hospital overnight or longer in the past 6 months? times

HCU_4. How many total NIGHTS did you spend in the hospital in the past 6 months? nights 


\section{Multiple Primary Cancer (MPC) Items - Part 2}

MPC_9. What do you wish every healthcare provider knew about what it's like to be diagnosed with cancer more than once? Please feel free to attach additional sheets if more space is needed. 


\section{You have completed all questionnaires.}

\section{A sincere thank you for being a part of this study!}

Please return 1) this packet of questionnaires and 2) one signed copy of the consent form to Sarah Belcher in the enclosed postage-paid envelope.

The second consent form copy is for your records.

You will be receiving instructions from our research team for accessing your compensation.

If you have any questions or concerns, please contact the study's principal investigator,

Sarah Belcher, at 412-624-8938 orLAMPstudy@pitt.edu.

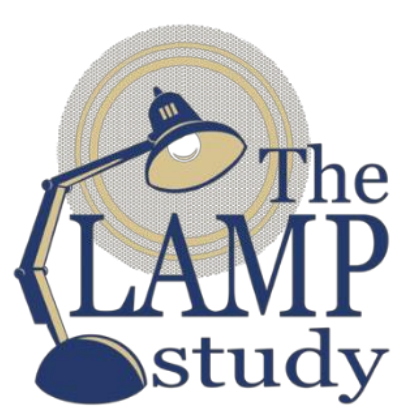




\section{Medical Record Data Extraction Form}

\begin{tabular}{|l|l}
\hline Participant Study ID: & Medical Record Number:
\end{tabular}

Data Collector Name:

Data Collection Date (mm/dd/yyyy):

\section{Participant Socio-Demographics}

Insurance: Record types from up to 1 year prior to $1^{\text {st }}$ cancer diagnosis to present.

\begin{tabular}{|l|l|}
\hline Insurance Type(s): & $\begin{array}{c}\text { Date(s) Recorded in Medical Record } \\
\text { (mm/dd/yyyy) }\end{array}$ \\
\hline \multirow{5}{*}{ (m) } & \\
\hline & \\
\hline & \\
\hline & \\
\hline
\end{tabular}

Anthropometric Data

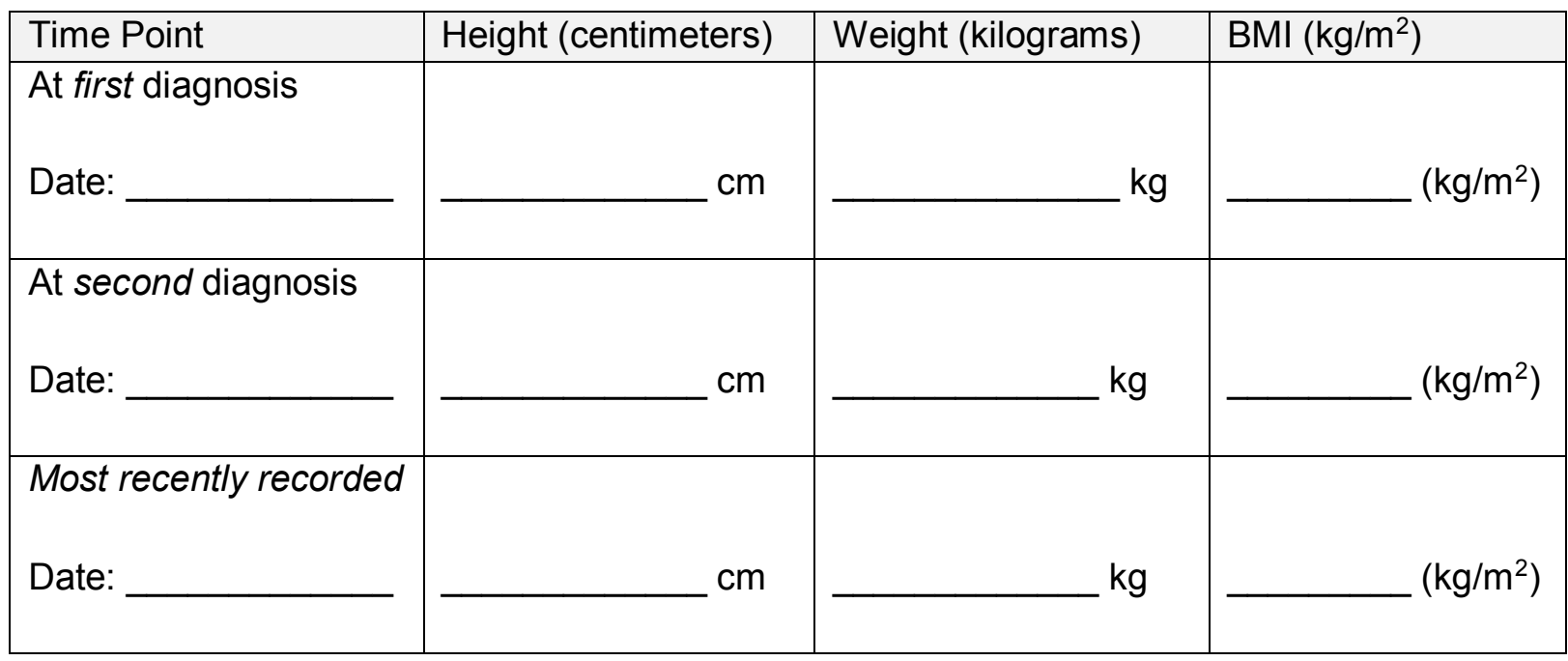




\section{Family Cancer History}

Family history of cancer in $1^{\text {st }}$ degree relative (parent, brother, sister, or child)?

$\square$ Yes $\square$ No If yes, list:

\begin{tabular}{|l|l|}
\hline Relationship: & Type of Cancer: \\
\hline & \\
\hline & \\
\hline & \\
\hline & \\
\hline & \\
\hline & \\
\hline & \\
\hline
\end{tabular}

\section{Genetic Testing}

Did participant receive any type of genetic testing?

$\square$ Yes $\square$ No If yes, describe:

\begin{tabular}{|l|l|}
\hline Date: & Type/Findings: \\
\hline & \\
\hline & \\
\hline & \\
\hline & \\
\hline & \\
\hline & \\
\hline & \\
\hline
\end{tabular}


Most Current Medication List (prescription and OTC):

Date Recorded in Medical Record (mm/dd/yyyy):

aspirin

atorvastatin (Lipitor)

diphenhydramine hydrochloride (Benadryl)

famotidine (Pepcid)

furosemide (Lasix)

hydrochlorothiazide (Microzide)

hydrocodone/acetaminophen (Vicodin, Norco, Xodol)

$\square$ levothyroxine (Synthroid, Levoxyl, Unithyroid)

$\square$ lisinopril (Prinivil, Zestril)

meclizine hydrochloride (Bonine, Verticalm)

metformin hydrochloride (Glucophage)

metoprolol (Lopressor, Toprol XL)

omeprazole (Prilosec)

ondansetron (Zofran)

prednisone (Delasone, Sterapred)

ranitidine (Zantac)

sertraline hydrochloride (Zoloft)

simvastin (Zocor, FloLipid)

zolpidem tartrate (Ambien)
Other (specify on lines below):

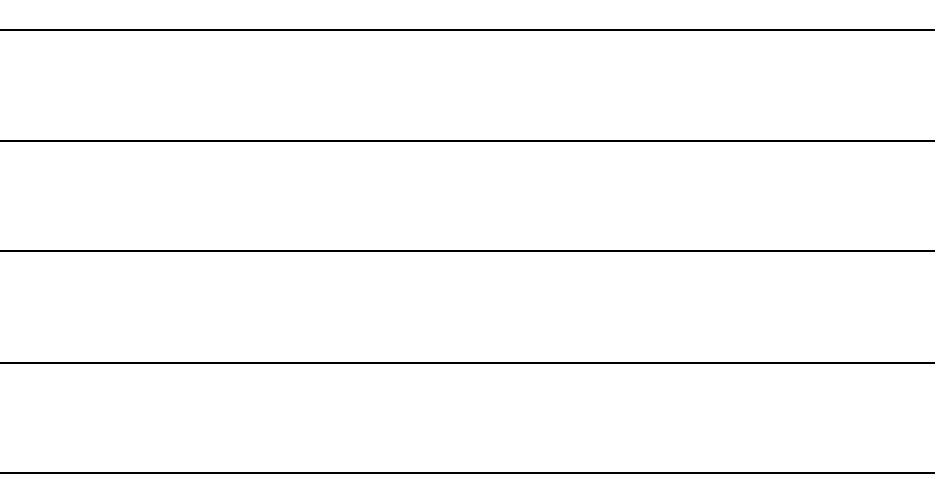


Non-Cancer Past Medical/Surgical History (Comorbidities):

Include non-invasive skin cancer (e.g. basal cell carcinoma) here if applicable.

Arthritis

Other (specify below):

Cardiac Disease (If yes, describe below:)

\section{Depression}

Diabetes

Dyslipidemia

Hypertension

Hypothyroidism

Menopause

Obesity

Osteoporosis \&/or Osteopenia

\section{Cancer History:}

Number of primary cancer diagnoses, excluding non-invasive skin cancers (e.g. basal cell).

Do not count metastases or recurrences!

primary cancer diagnoses

Were primary cancer diagnoses:
Synchronous (at same time)?
$\square$ Metachronous (at different times)?

Relevant notes: 
First Primary Cancer Type:

Note: Exclude cases of non-invasive skin cancer (list in past medical/surgical history).

Breast Cancer

Prostate Cancer

Colorectal Cancer

Urinary Bladder Cancer

Uterine Corpus Cancer

Melanoma
Kidney / Renal Pelvis Cancer

Oral Cavity / Pharynx Cancer

Lung / Bronchus Cancer

Thyroid Cancer

Ovarian Cancer

Other (specify below):

Date of Diagnosis:

Histology:

Cancer Stage at first cancer diagnosis:

Did the first cancer recur?

$\square$ Yes $\square$ No

If yes: List number of total recurrences: (describe in table below)

\begin{tabular}{|l|l|l|}
\hline Recurrence(s) & $\begin{array}{l}\text { Date: } \\
(\mathrm{mm} / \mathrm{dd} / \mathrm{yyyy})\end{array}$ & Site(s) of Recurrent/Metastatic Disease: \\
\hline 1st Recurrence & & \\
\hline $2^{\text {nd }}$ Recurrence & & \\
\hline $3^{\text {nd }}$ Recurrence & & \\
\hline $4^{\text {th }}$ Recurrence & & \\
\hline
\end{tabular}



Cancer Treatment Type(s) Received for First Cancer:
$\square$ Surgery, If yes, describe below
Targeted Therapy
$\square$ Radiation Therapy
$\square$ Hormone Therapy
Chemotherapy
Stem Cell Transplant
$\square$ Immunotherapy
Other:

Date of Treatment Initiation for First Primary Cancer:

Date of Treatment Completion for First Primary Cancer: $\quad$ (check here if treatment ongoing: $\bullet$ )

Note Frontline Therapy Received for First Primary Cancer Below:

(e.g. FOLFOX 32mg/m2, every 6 weeks $x$ cycles; 6 cycles received; last dose held due to grade 4 neuropathy; Radiation therapy doses of 75.6-79 Gy in conventional 36-41 Gy fractions to the prostate-with 3D-CRT/IMRT with daily image-guided radiotherapy (IGRT) or brachytherapy)

\begin{tabular}{|l|c|c|c|c|c|c|}
\hline $\begin{array}{c}\text { Regimen } \\
\text { Name(s) }\end{array}$ & $\begin{array}{c}\text { Individual Drug } \\
\text { Name(s) and } \\
\text { Prescribed Dosages }\end{array}$ & $\begin{array}{c}\text { Time } \\
\text { Interval/ } \\
\text { Frequency }\end{array}$ & $\begin{array}{c}\text { Dosage } \\
\text { Received }\end{array}$ & $\begin{array}{c}\text { Dose } \\
\text { Delay? } \\
\text { (Y or N) }\end{array}$ & $\begin{array}{c}\text { Dose } \\
\text { Reduction? } \\
\text { (Y or N) }\end{array}$ & $\begin{array}{c}\text { Additional Notes } \\
\text { (include regimen } \\
\text { alteration rationale) }\end{array}$ \\
\hline & & & & & & \\
\hline & & & & & & \\
\hline
\end{tabular}

Note Subsequent Lines of Therapy (e.g. palliative, salvage intent) Received for First Primary Cancer Below:

\begin{tabular}{|l|l|l|l|l|}
\hline & $\begin{array}{c}\text { Individual Drug Name(s) } \\
\text { and Prescribed Dosages }\end{array}$ & $\begin{array}{c}\text { Time Interval/ } \\
\text { Frequency }\end{array}$ & $\begin{array}{c}\text { \# Cycles } \\
\text { Received }\end{array}$ & $\begin{array}{c}\text { Additional Notes } \\
\text { (include regimen } \\
\text { alteration rationale) }\end{array}$ \\
\hline & & & & \\
\hline & & & & \\
\hline & & & & \\
\hline & & & & \\
\hline
\end{tabular}




\section{Second Primary Cancer Type:}

Note: Exclude cases of non-invasive skin cancer (list in past medical/surgical history).

Breast Cancer

Prostate Cancer

Colorectal Cancer

Urinary Bladder Cancer

Uterine Corpus Cancer

Melanoma
Kidney / Renal Pelvis Cancer

Oral Cavity / Pharynx Cancer

$\square$ Lung / Bronchus Cancer

Thyroid Cancer

Ovarian Cancer

Other (specify below):

Date of Diagnosis:

Histology:

Cancer Stage at second cancer diagnosis:

Did the second cancer recur?
Yes
$\square$ No

If yes: List number of total recurrences: (describe in table below)

\begin{tabular}{|l|l|l|}
\hline Recurrence(s) & $\begin{array}{l}\text { Date: } \\
\text { (mm/dd/yyyy) }\end{array}$ & Site(s) of Recurrent/Metastatic Disease: \\
\hline 1st Recurrence & & \\
\hline $2^{\text {nd }}$ Recurrence & & \\
\hline $3^{\text {nd }}$ Recurrence & & \\
\hline $4^{\text {th }}$ Recurrence & & \\
\hline
\end{tabular}


Cancer Treatment Type(s) Received for Second Cancer:

$\square$ Surgery, If yes, describe below

Targeted Therapy

Radiation Therapy

Chemotherapy

$\square$ Immunotherapy
Hormone Therapy

Stem Cell Transplant

Other:

Date of Treatment Initiation for Second Primary Cancer:

Date of Treatment Completion for Second Primary Cancer: $\quad$ (check here if treatment ongoing: $\bullet$ )

Note Frontline Therapy Received for Second Primary Cancer Below:

(e.g. FOLFOX 32mg/m2, every 6 weeks x 6 cycles; 6 cycles received; last dose held due to grade 4 neuropathy; Radiation therapy doses of 75.6-79 Gy in conventional 36-41 Gy fractions to the prostate-with 3D-CRT/IMRT with daily image-guided radiotherapy (IGRT) or brachytherapy)

\begin{tabular}{|l|c|c|c|c|c|c|}
\hline $\begin{array}{l}\text { Regimen } \\
\text { Name(s) }\end{array}$ & $\begin{array}{c}\text { Individual Drug } \\
\text { Name(s) and } \\
\text { Prescribed Dosages }\end{array}$ & $\begin{array}{c}\text { Time } \\
\text { Interval/ } \\
\text { Frequency }\end{array}$ & $\begin{array}{c}\text { Dosage } \\
\text { Received }\end{array}$ & $\begin{array}{c}\text { Dose } \\
\text { Delay? } \\
\text { (Y or N) }\end{array}$ & $\begin{array}{c}\text { Dose } \\
\text { Reduction? } \\
\text { (Y or N) }\end{array}$ & $\begin{array}{c}\text { Additional Notes } \\
\text { (include regimen } \\
\text { alteration rationale) }\end{array}$ \\
\hline & & & & & & \\
\hline
\end{tabular}

Note Subsequent Lines of Therapy (e.g. palliative, salvage intent) Received for Second Primary Cancer Below:

\begin{tabular}{|l|l|l|l|l|}
\hline Regimen Name(s) & $\begin{array}{c}\text { Individual Drug Name(s) } \\
\text { and Prescribed Dosages }\end{array}$ & $\begin{array}{c}\text { Time Interval/ } \\
\text { Frequency }\end{array}$ & $\begin{array}{c}\text { \# Cycles } \\
\text { Received }\end{array}$ & $\begin{array}{c}\text { Additional Notes } \\
\text { (include regimen } \\
\text { alteration rationale) }\end{array}$ \\
\hline & & & & \\
\hline & & & & \\
\hline & & & & \\
\hline
\end{tabular}




\section{Third Primary Cancer Type:}

\section{Note: Exclude cases of non-invasive skin cancer (list in past medical/surgical history).}

Breast Cancer

Prostate Cancer

Colorectal Cancer

Urinary Bladder Cancer

Uterine Corpus Cancer

Melanoma
Kidney / Renal Pelvis Cancer

Oral Cavity / Pharynx Cancer

$\square$ Lung / Bronchus Cancer

Thyroid Cancer

Ovarian Cancer

Other (specify below):

Date of Diagnosis:

Histology:

Cancer Stage at third cancer diagnosis:

Did the third cancer recur?

Yes $\square$ No

If yes: List number of total recurrences:

(describe in table below)

\begin{tabular}{|l|l|l|}
\hline Recurrence(s) & $\begin{array}{l}\text { Date: } \\
(\mathrm{mm} / \mathrm{dd} / \mathrm{yyyy})\end{array}$ & Site(s) of Recurrent/Metastatic Disease: \\
\hline 1st Recurrence & & \\
\hline $2^{\text {nd }}$ Recurrence & & \\
\hline $3^{\text {nd }}$ Recurrence & & \\
\hline $4^{\text {th }}$ Recurrence & & \\
\hline
\end{tabular}


Cancer Treatment Type(s) Received for Third Cancer:

$\square$ Surgery, If yes, describe below

Targeted Therapy

Radiation Therapy

Chemotherapy

$\square$ Immunotherapy
Hormone Therapy

Stem Cell Transplant

Other:

Date of Treatment Initiation for Third Primary Cancer:

Date of Treatment Completion for Third Primary Cancer: $\quad$ (check here if treatment ongoing: •)

Note Frontline Therapy Received for Third Primary Cancer Below:

(e.g. FOLFOX 32mg/m2, every 6 weeks $x$ cycles; 6 cycles received; last dose held due to grade 4 neuropathy; Radiation therapy doses of 75.6-79 Gy in conventional 36-41 Gy fractions to the prostate-with 3D-CRT/IMRT with daily image-guided radiotherapy (IGRT) or brachytherapy)

\begin{tabular}{|l|c|c|c|c|c|c|}
\hline $\begin{array}{l}\text { Regimen } \\
\text { Name(s) }\end{array}$ & $\begin{array}{c}\text { Individual Drug } \\
\text { Name(s) and } \\
\text { Prescribed Dosages }\end{array}$ & $\begin{array}{c}\text { Time } \\
\text { Interval/ } \\
\text { Frequency }\end{array}$ & $\begin{array}{c}\text { Dosage } \\
\text { Received }\end{array}$ & $\begin{array}{c}\text { Dose } \\
\text { Delay? } \\
\text { (Y or N) }\end{array}$ & $\begin{array}{c}\text { Dose } \\
\text { Reduction? } \\
\text { (Y or N) }\end{array}$ & $\begin{array}{c}\text { Additional Notes } \\
\text { (include regimen } \\
\text { alteration rationale) }\end{array}$ \\
\hline & & & & & & \\
\hline & & & & & & \\
\hline
\end{tabular}

Note Subsequent Lines of Therapy (e.g. palliative, salvage intent) Received for Third Primary Cancer Below:

\begin{tabular}{|l|l|l|l|l|}
\hline Regimen Name(s) & $\begin{array}{c}\text { Individual Drug Name(s) } \\
\text { and Prescribed Dosages }\end{array}$ & $\begin{array}{c}\text { Time Interval/ } \\
\text { Frequency }\end{array}$ & $\begin{array}{c}\text { \# Cycles } \\
\text { Received }\end{array}$ & $\begin{array}{c}\text { Additional Notes } \\
\text { (include regimen } \\
\text { alteration rationale) }\end{array}$ \\
\hline & & & & \\
\hline & & & & \\
\hline & & & & \\
\hline & & & & \\
\hline
\end{tabular}


Fourth Primary Cancer Type:

Note: Exclude cases of non-invasive skin cancer (list in past medical/surgical history).

Breast Cancer

Prostate Cancer

Colorectal Cancer

Urinary Bladder Cancer

Uterine Corpus Cancer

Melanoma
Kidney / Renal Pelvis Cancer

Oral Cavity / Pharynx Cancer

$\square$ Lung / Bronchus Cancer

Thyroid Cancer

Ovarian Cancer

Other (specify below):

Date of Diagnosis:

Histology:

Cancer Stage at fourth cancer diagnosis:

Did the fourth cancer recur?

Yes $\square$ No

If yes: List number of total recurrences: (describe in table below)

\begin{tabular}{|l|l|l|}
\hline Recurrence(s) & $\begin{array}{l}\text { Date: } \\
\text { (mm/dd/yyyy) }\end{array}$ & Site(s) of Recurrent/Metastatic Disease: \\
\hline 1st Recurrence & & \\
\hline $2^{\text {nd }}$ Recurrence & & \\
\hline $3^{\text {nd }}$ Recurrence & & \\
\hline $4^{\text {th }}$ Recurrence & & \\
\hline
\end{tabular}


Cancer Treatment Type(s) Received for Fourth Cancer:

$\square$ Surgery, If yes, describe below

Targeted Therapy

Radiation Therapy

Chemotherapy

$\square$ Immunotherapy $\square$ Hormone Therapy

Stem Cell Transplant

Other:

Date of Treatment Initiation for Fourth Primary Cancer:

Date of Treatment Completion for Fourth Primary Cancer: $\quad$ (check here if treatment ongoing: •)

Note Frontline Therapy Received for Fourth Primary Cancer Below:

(e.g. FOLFOX 32mg/m2, every 6 weeks $x 6$ cycles; 6 cycles received; last dose held due to grade 4 neuropathy; Radiation therapy doses of 75.6-79 Gy in conventional 36-41 Gy fractions to the prostate-with 3D-CRT/IMRT with daily image-guided radiotherapy (IGRT) or brachytherapy)

\begin{tabular}{|l|c|c|c|c|c|c|}
\hline $\begin{array}{c}\text { Regimen } \\
\text { Name(s) }\end{array}$ & $\begin{array}{c}\text { Individual Drug } \\
\text { Name(s) and } \\
\text { Prescribed Dosages }\end{array}$ & $\begin{array}{c}\text { Time } \\
\text { Interval/ } \\
\text { Frequency }\end{array}$ & $\begin{array}{c}\text { Dosage } \\
\text { Received }\end{array}$ & $\begin{array}{c}\text { Dose } \\
\text { Delay? } \\
\text { (Y or N) }\end{array}$ & $\begin{array}{c}\text { Dose } \\
\text { Reduction? } \\
\text { (Y or N) }\end{array}$ & $\begin{array}{c}\text { Additional Notes } \\
\text { (include regimen } \\
\text { alteration rationale) }\end{array}$ \\
\hline & & & & & & \\
\hline & & & & & & \\
\hline
\end{tabular}

Note Subsequent Lines of Therapy (e.g. palliative, salvage intent) Received for Fourth Primary Cancer Below:

\begin{tabular}{|l|l|l|l|l|}
\hline & $\begin{array}{c}\text { Individual Drug Name(s) } \\
\text { and Prescribed Dosages }\end{array}$ & $\begin{array}{c}\text { Time Interval/ } \\
\text { Frequency }\end{array}$ & $\begin{array}{c}\text { \# Cycles } \\
\text { Received }\end{array}$ & $\begin{array}{c}\text { Additional Notes } \\
\text { (include regimen } \\
\text { alteration rationale) }\end{array}$ \\
\hline & & & & \\
\hline & & & & \\
\hline & & & & \\
\hline & & & & \\
\hline
\end{tabular}




\section{$\underline{\text { UPMC Cancer Registry Data }}$}

Hospital ID:

Managing Physician

of Record (Last, First):

Surgeon of Record

(Last, First):

Date of Last Contact

(mm/dd/yyyy):

Cancer Registry - Cancer Data:

\begin{tabular}{|l|l|}
\hline \multicolumn{2}{|l|}{ First Primary Cancer: } \\
\hline Diagnosis 1 & \\
\hline Date of Diagnosis 1 (mm/dd/yyyy) & \\
\hline Laterality & \\
\hline Histology & \\
\hline Stage at Diagnosis & \\
\hline
\end{tabular}

\begin{tabular}{|l|l|}
\hline Second Primary Cancer: & \\
\hline Diagnosis 2 & \\
\hline Date of Diagnosis 2 (mm/dd/yyyy) & \\
\hline Laterality & \\
\hline Histology & \\
\hline Stage at Diagnosis & \\
\hline
\end{tabular}




\begin{tabular}{|l|l|}
\hline \multicolumn{2}{|l|}{ Third Primary Cancer: } \\
\hline Diagnosis 3 & \\
\hline Date of Diagnosis 3 (mm/dd/yyyy) & \\
\hline Laterality & \\
\hline Histology & \\
\hline Stage at Diagnosis & \\
\hline
\end{tabular}

\begin{tabular}{|l|l|}
\hline \multicolumn{2}{|l|}{ Fourth Primary Cancer: } \\
\hline Diagnosis 4 & \\
\hline Date of Diagnosis 4 (mm/dd/yyyy) & \\
\hline Laterality & \\
\hline Histology & \\
\hline Stage at Diagnosis & \\
\hline
\end{tabular}


APPENDIX C

HUMAN SUBJECTS APPROVALS AND CONSENTS (PR016050542) 
Pitt Seal

\section{University of Pittsburgh Institutional Review Board}

3500 Fifth Avenue

Pittsburgh, PA 15213

(412) 383-1480

(412) 383-1508 (fax)

http://www.irb.pitt.edu

\section{$\underline{\text { Memorandum }}$}

To: $\quad$ Sarah Belcher

From: IRB Office

Date: $\quad 10 / 3 / 2017$

IRB\#: $\quad \underline{\text { PRO16050542 }}$

Subject: Characterizing Psychobehavioral Risks in Survivors of Multiple Primary Cancers

The University of Pittsburgh Institutional Review Board reviewed and approved the above referenced study by the expedited review procedure authorized under 45 CFR 46.110 and 21 CFR 56.110. Your research study was approved under:

45 CFR 46.110.(5)

45 CFR 46.110.(7)

The risk level designation is Minimal Risk.

Approval Date: $\quad 10 / 3 / 2017$

Expiration Date: $10 / 2 / 2018$

For studies being conducted in UPMC facilities, no clinical activities can be undertaken by investigators until they have received approval from the UPMC Fiscal Review Office.

Please note that it is the investigator's responsibility to report to the IRB any unanticipated problems involving risks to subjects or others [see 45 CFR 46.103(b)(5) and 21 CFR 56.108(b)]. Refer to the IRB Policy and Procedure Manual regarding the reporting requirements for unanticipated problems which include, but are not limited to, adverse events. If you have any questions about this process, please contact the Adverse Events Coordinator at 412-383-1480.

The protocol and consent forms, along with a brief progress report must be resubmitted at least one month prior to the renewal date noted above as required by FWA00006790 (University of Pittsburgh), FWA00006735 (University of Pittsburgh Medical Center), FWA00000600 (Children's Hospital of Pittsburgh), FWA00003567 (Magee-Womens Health Corporation), FWA00003338 (University of 
Pittsburgh Medical Center Cancer Institute).

Please be advised that your research study may be audited periodically by the University of Pittsburgh Research Conduct and Compliance Office. 
Pitt Seal

\section{University of Pittsburgh Institutional Review Board}

\section{$\underline{\text { Memorandum }}$}

To: $\quad$ Sarah Belcher

From: IRB Office

Date: $\quad 10 / 17 / 2017$

IRB\#: $\quad$ MOD16050542-01 / PRO16050542

Subject: Characterizing Psychobehavioral Risks in Survivors of Multiple Primary Cancers

The University of Pittsburgh Institutional Review Board reviewed and approved the requested modifications by expedited review procedure authorized under 45 CFR 46.110 and 21 CFR 56.110.

Modification Approval Date: 10/17/2017

Expiration Date: $\quad$ 10/2/2018

For studies being conducted in UPMC facilities, no clinical activities that are impacted by the modifications can be undertaken by investigators until they have received approval from the UPMC Fiscal Review Office.

Please note that it is the investigator's responsibility to report to the IRB any unanticipated problems involving risks to subjects or others [see 45 CFR 46.103(b)(5) and 21 CFR 56.108(b)]. Refer to the IRB Policy and Procedure Manual regarding the reporting requirements for unanticipated problems which include, but are not limited to, adverse events. If you have any questions about this process, please contact the Adverse Events Coordinator at 412-383-1480.

The protocol and consent forms, along with a brief progress report must be resubmitted at least one month prior to the renewal date noted above as required by FWA00006790 (University of Pittsburgh), FWA00006735 (University of Pittsburgh Medical Center), FWA00000600 (Children's Hospital of Pittsburgh), FWA00003567 (Magee-Womens Health Corporation), FWA00003338 (University of Pittsburgh Medical Center Cancer Institute).

Please be advised that your research study may be audited periodically by the University of Pittsburgh Research Conduct and Compliance Office. 
Pitt Seal

\section{University of Pittsburgh Institutional Review Board}

\section{$\underline{\text { Memorandum }}$}

To: $\quad$ Sarah Belcher

From: IRB Office

Date: $\quad 12 / 7 / 2017$

IRB\#: $\quad$ MOD16050542-02 / PRO16050542

Subject: Characterizing Psychobehavioral Risks in Survivors of Multiple Primary Cancers

The University of Pittsburgh Institutional Review Board reviewed and approved the requested modifications by expedited review procedure authorized under 45 CFR 46.110 and 21 CFR 56.110.

Modification Approval Date: 12/7/2017

Expiration Date: $\quad$ 10/2/2018

For studies being conducted in UPMC facilities, no clinical activities that are impacted by the modifications can be undertaken by investigators until they have received approval from the UPMC Fiscal Review Office.

Please note that it is the investigator's responsibility to report to the IRB any unanticipated problems involving risks to subjects or others [see 45 CFR 46.103(b)(5) and 21 CFR 56.108(b)]. Refer to the IRB Policy and Procedure Manual regarding the reporting requirements for unanticipated problems which include, but are not limited to, adverse events. If you have any questions about this process, please contact the Adverse Events Coordinator at 412-383-1480.

The protocol and consent forms, along with a brief progress report must be resubmitted at least one month prior to the renewal date noted above as required by FWA00006790 (University of Pittsburgh), FWA00006735 (University of Pittsburgh Medical Center), FWA00000600 (Children's Hospital of Pittsburgh), FWA00003567 (Magee-Womens Health Corporation), FWA00003338 (University of Pittsburgh Medical Center Cancer Institute).

Please be advised that your research study may be audited periodically by the University of Pittsburgh Research Conduct and Compliance Office. 
Pitt Seal

\section{University of Pittsburgh Institutional Review Board}

\section{$\underline{\text { Memorandum }}$}

To: $\quad$ Sarah Belcher

From: IRB Office

Date: $\quad 12 / 20 / 2017$

IRB\#: $\quad$ MOD16050542-03 / PRO16050542

Subject: Characterizing Psychobehavioral Risks in Survivors of Multiple Primary Cancers

The University of Pittsburgh Institutional Review Board reviewed and approved the requested modifications by expedited review procedure authorized under 45 CFR 46.110 and 21 CFR 56.110.

Modification Approval Date: 12/20/2017

Expiration Date: $\quad$ 10/2/2018

For studies being conducted in UPMC facilities, no clinical activities that are impacted by the modifications can be undertaken by investigators until they have received approval from the UPMC Fiscal Review Office.

Please note that it is the investigator's responsibility to report to the IRB any unanticipated problems involving risks to subjects or others [see 45 CFR 46.103(b)(5) and 21 CFR 56.108(b)]. Refer to the IRB Policy and Procedure Manual regarding the reporting requirements for unanticipated problems which include, but are not limited to, adverse events. If you have any questions about this process, please contact the Adverse Events Coordinator at 412-383-1480.

The protocol and consent forms, along with a brief progress report must be resubmitted at least one month prior to the renewal date noted above as required by FWA00006790 (University of Pittsburgh), FWA00006735 (University of Pittsburgh Medical Center), FWA00000600 (Children's Hospital of Pittsburgh), FWA00003567 (Magee-Womens Health Corporation), FWA00003338 (University of Pittsburgh Medical Center Cancer Institute).

Please be advised that your research study may be audited periodically by the University of Pittsburgh Research Conduct and Compliance Office. 


\section{University of Pittsburgh Institutional Review Board}

\section{$\underline{\text { Memorandum }}$}

To: $\quad$ Sarah Belcher

From: IRB Office

Date: $\quad 2 / 8 / 2018$

IRB\#: $\quad$ MOD16050542-04 / PRO16050542

Subject: Characterizing Psychobehavioral Risks in Survivors of Multiple Primary Cancers

The University of Pittsburgh Institutional Review Board reviewed and approved the requested modifications by the expedited review procedure authorized under 45 CFR 46.110 and 21 CFR 56.110.

Modification Approval Date: 2/8/2018

Expiration Date: $\quad$ 10/2/2018

[see 45 CFR 46.103(b)(5) and 21 CFR 56.108(b)]. Refer to the IRB Policy and Procedure Manual regarding the reporting requirements for unanticipated problems which include, but are not limited to, adverse events. If you have any questions about this process, please contact the Adverse Events Coordinator at 412-383-1480.

The protocol and consent forms, along with a brief progress report must be resubmitted at least one month prior to the renewal date noted above as required by FWA00006790 (University of Pittsburgh), FWA00006735 (University of Pittsburgh Medical Center), FWA00000600 (Children's Hospital of Pittsburgh), FWA00003567 (Magee-Womens Health Corporation), FWA00003338 (University of Pittsburgh Medical Center Cancer Institute).

Please be advised that your research study may be audited periodically by the University of Pittsburgh Research Conduct and Compliance Office. 


\section{University of Pittsburgh Institutional Review Board}

\section{$\underline{\text { Memorandum }}$}

To: $\quad$ Sarah Belcher

From: IRB Office

Date: $\quad 3 / 13 / 2018$

IRB\#： $\quad$ MOD16050542-06 / PRO16050542

Subject: Characterizing Psychobehavioral Risks in Survivors of Multiple Primary Cancers

The University of Pittsburgh Institutional Review Board reviewed and approved the requested modifications by expedited review procedure authorized under 45 CFR 46.110 and 21 CFR 56.110.

Modification Approval Date: 3/13/2018

Expiration Date: $\quad 10 / 2 / 2018$

For studies being conducted in UPMC facilities, no clinical activities that are impacted by the modifications can be undertaken by investigators until they have received approval from the UPMC Fiscal Review Office.

Please note that it is the investigator's responsibility to report to the IRB any unanticipated problems involving risks to subjects or others [see 45 CFR 46.103(b)(5) and 21 CFR 56.108(b)]. Refer to the IRB Policy and Procedure Manual regarding the reporting requirements for unanticipated problems which include, but are not limited to, adverse events. If you have any questions about this process, please contact the Adverse Events Coordinator at 412-383-1480.

The protocol and consent forms, along with a brief progress report must be resubmitted at least one month prior to the renewal date noted above as required by FWA00006790 (University of Pittsburgh), FWA00006735 (University of Pittsburgh Medical Center), FWA00000600 (Children's Hospital of Pittsburgh), FWA00003567 (Magee-Womens Health Corporation), FWA00003338 (University of Pittsburgh Medical Center Cancer Institute).

Please be advised that your research study may be audited periodically by the University of Pittsburgh Research Conduct and Compliance Office. 


\section{University of Pittsburgh Institutional Review Board}

\section{$\underline{\text { Memorandum }}$}

To: $\quad$ Sarah Belcher

From: IRB Office

Date: $\quad 3 / 16 / 2018$

IRB\#： $\quad$ MOD16050542-07 / PRO16050542

Subject: Characterizing Psychobehavioral Risks in Survivors of Multiple Primary Cancers

The University of Pittsburgh Institutional Review Board reviewed and approved the requested modifications by expedited review procedure authorized under 45 CFR 46.110 and 21 CFR 56.110.

Modification Approval Date: 3/16/2018

Expiration Date: $\quad 10 / 2 / 2018$

For studies being conducted in UPMC facilities, no clinical activities that are impacted by the modifications can be undertaken by investigators until they have received approval from the UPMC Fiscal Review Office.

Please note that it is the investigator's responsibility to report to the IRB any unanticipated problems involving risks to subjects or others [see 45 CFR 46.103(b)(5) and 21 CFR 56.108(b)]. Refer to the IRB Policy and Procedure Manual regarding the reporting requirements for unanticipated problems which include, but are not limited to, adverse events. If you have any questions about this process, please contact the Adverse Events Coordinator at 412-383-1480.

The protocol and consent forms, along with a brief progress report must be resubmitted at least one month prior to the renewal date noted above as required by FWA00006790 (University of Pittsburgh), FWA00006735 (University of Pittsburgh Medical Center), FWA00000600 (Children's Hospital of Pittsburgh), FWA00003567 (Magee-Womens Health Corporation), FWA00003338 (University of Pittsburgh Medical Center Cancer Institute).

Please be advised that your research study may be audited periodically by the University of Pittsburgh Research Conduct and Compliance Office. 


\section{University of Pittsburgh \\ School of Nursing \\ Department of Health and Community Systems}

CONSENT TO ACT AS A PARTICIPANT IN A RESEARCH STUDY

TITLE OF RESEARCH PROJECT:

Characterizing Psychobehavioral Risks in Survivors of Multiple Primary Cancers: The LAMP Study

PRINCIPAL INVESTIGATOR: $\quad$ Sarah M. Belcher, PhD(c), RN, OCN ${ }^{\circledR}$

Doctoral Candidate

University of Pittsburgh School of Nursing

415 Victoria Building

3500 Victoria Street

Pittsburgh, PA 15261

412-624-8938

\section{CO-INVESTIGATORS:}

Leonard Appleman, MD, PhD

Physican, Division of Hematology-Oncology

UPMC

412-648-6507

Adam Brufsky, MD, PhD

Associate Chief, Division of Hematology-Oncology

Co-Director, Comprehensive Breast Center

UPMC

412-641-6500

Edward Chu, MD

Chief, Division of Hematology-Oncology

UPMC

412-648-6589

Robert Edwards, MD

Chair, Gynecology \& Reproductive Sciences

Magee-Womens Hospital of UPMC

412-641-4212

John Kirkwood, MD

Director, Melanoma and Skin Cancer Program

UPMC

412-623-7707
Dana Bovbjerg, PhD

Director, Biobehavioral Oncology Program University of Pittsburgh Cancer Institute 412-623-5965

Grace Campbell, PhD, MSW, RN, CRRN

Assistant Professor

School of Nursing

412-417-8804

Heidi Donovan, PhD, RN

Professor and Vice Chair for Research

School of Nursing

412-624-2699

Jonas Johnson, MD

Chairman, Otolaryngology

UPMC

412-647-2100

Susan Sereika, PhD

Director, Center for Research and Evaluation School of Nursing

412-624-0799 
Paula Sherwood, PhD, RN, CNRN, FAAN

Professor

School of Nursing

412-624-4802
Liza Villaruz, MD

Physician, Division of Hematology-Oncology UPMC

412-648-6578

SOURCES OF SUPPORT: American Cancer Society Doctoral Degree Scholarship in Cancer Nursing Robert Wood Johnson Foundation Future of Nursing Scholars Nightingale Awards of Pennsylvania University of Pittsburgh School of Nursing

You are being asked to take part in a research study being conducted by the University of Pittsburgh's School of Nursing. We will ask you to complete a one-time set of questionnaires that will take approximately 30-40 minutes to complete.

\section{WHY IS THIS RESEARCH BEING DONE?}

We are doing this study to help us understand the experiences of adult cancer survivors like you who have had two separate types of cancer. We call two or more separate types of cancers "multiple primary cancers." We want to learn more about how having multiple primary cancers impacts stress, health behaviors, and emotional and overall health.

\section{WHO IS BEING ASKED TO TAKE PART IN THIS RESEARCH STUDY?}

You are being invited to take part in this research study because you have a history of two separate types of cancer, or multiple primary cancers. We will enroll 450 multiple primary cancer survivors like you in this study.

\section{WHAT PROCEDURES WILL BE PERFORMED FOR RESEARCH PURPOSES?}

The study procedures consist of a one-time completion of a set of questionnaires and a medical record review. We will send you either a personalized internet link to complete the questionnaire online or a pencil-paper copy of the questionnaires with a pre-stamped, pre-addressed return envelope.

1. The questionnaire can be completed online, or we can mail you the questionnaire to do on paper. You will complete a set of questionnaires that include demographic and health information and questions about stress, health behaviors, your experience with cancer, and emotional and overall health.

If done on paper, you will then return the signed consent form (required) along with the completed questionnaires in the return envelope provided to you.

Online responses are automatically recorded.

We estimate that the questionnaire will take 30-40 minutes to complete.

Page 2 of 5 
If we do not receive your questionnaires after we sent you the initial packet in the mail, we will send you a reminder postcard two weeks after the initial letter and a reminder letter and replacement questionnaire three weeks after the initial letter.

2. We will review your medical record to find out about your health.

We will record information about types of cancer, dates diagnosed, cancer treatments, and any other illnesses you may have had.

3. A refusal form is requested if you do not want to participate in the study.

If you choose not to participate in the study, you will be asked to complete five questions to help us understand why people are not participating in this study. Answering these additional five questions is voluntary.

\section{WHAT ARE THE POSSIBLE RISKS, SIDE EFFECTS, AND DISCOMFORTS OF THIS RESEARCH STUDY?}

This is a very low risk study, but you should be aware of risks.

1. One potential risk is a breach of confidentiality, but we will do everything possible to protect your privacy. To protect your privacy, only Ms. Belcher (the principle investigator) and members of the research team will be aware of your participation in this research study. Your name will not be included on the questionnaires we ask you to complete or the information we collect from your medical record. Mailed questionnaires and information collected about you from your medical record will be kept in secure, locked file cabinets at the School of Nursing. All information will be identified only by a study ID number. The information linking these ID numbers with your identify will be kept separate from the research records and will be stored under lock and key. If you complete the questionnaires online, the website where you complete the questionnaire is secure, and your data will be safely stored and can only be accessed by study team members. All researchers involved in this study have been thoroughly trained to maintain your privacy. All information you provide will be kept by the Principle Investigator in a locked file cabinet within a locked office at the School of Nursing. Your identity will not be revealed in any description or publications of this research, and data will only be presented about groups and not individual participants.

2. Another possible risk of this research study may include stress from having to complete the questionnaires. If the questions cause you stress or discomfort, you can take a break from completing the questionnaires. If any individual questions makes you feel distressed (anxious, sad, or nervous), you do not have to answer them.

\section{WHAT ARE POSSIBLE BENEFITS FROM TAKING PART IN THIS STUDY?}

You will likely receive no direct benefit from taking part in this research study. The results from this study may benefit survivors like you who experience multiple primary cancer diagnoses in the future but will have no direct benefit to you.

Page 3 of 5 


\section{WILL I BE PAID IF I TAKE PART IN THIS RESEARCH STUDY?}

Each participant will be provided with a $\$ 5$ Amazon.com gift card code in recognition of his or her time and expertise after completing and returning the survey to Ms. Belcher.

\section{WHO WILL KNOW ABOUT MY PARTICIPATION IN THIS RESEARCH STUDY?}

Any information about you obtained from this research will be kept strictly confidential (private), and any data that includes your identity will be stored in locked files in Research Project Office or in a password protected computer system. All records related to your involvement in this research study will be stored in a locked file cabinet in a locked room at the School of Nursing. Your identity on these records, and in the corresponding data entered into the computerized system, will be indicated by an ID number rather than by your name. The information linking these ID numbers with your identity will be kept separate from the research records. You will not be identified by name in any publication of the research. All records will be retained by us for a minimum of seven years.

It is possible that we may use the information obtained from this study to answer more research questions in other research studies. This information may also be shared with other researchers here, and at other research centers, but those researchers will never be provided with any personal identifiers that would allow them to learn who you are.

\section{Why is my authorization being requested?}

We are also requesting your authorization, or permission, to review your medical records to confirm information about your cancer and medical history and treatments. The authorization to access your medical records will be valid for a minimum of 7 years. We will obtain the following information: your diagnoses and treatments, age, past medical history, and results of any tissue biopsies or blood tests done as part of your standard evaluation at the Cancer Center. This identifiable medical record information will be made available to members of the research team for an indefinite period of time. Your medical information, as well as information obtained during this research study, may be shared with other groups, possibly including authorized officials from the University of Pittsburgh Research Conduct and Compliance Office, for the purpose of monitoring the study. Authorized representatives of UPMC or affiliated health care providers may also have access to this information to provide services and address billing and operational issues.

We will make every attempt to protect your privacy and the confidentiality of your records, as described in this document, but cannot guarantee the confidentiality of your research records, including information obtained from your medical records once your personal information is disclosed to others outside UPMC or the University. You can always withdraw your authorization to allow the research team to review your medical records by contacting the investigator listed on the first page and making the request in writing. If you do so, you will no longer be permitted to participate in this study. Any information obtained from you up to that point will continue to be used by the research team.

\section{IS MY PARTICIPATION IN THIS STUDY VOLUNTARY?}

Your participation in this study is completely voluntary. You may refuse to take part in it, or you may stop participating at any time, even after signing this form. Your decision will not affect your relationship or

Page 4 of 5

University Of Pittsburgh Institutional Review Board
Approval Date: «Approval Date» Renewal Date: «Renewal Date»
IRB \#: «IRBNo» 
current or future care at a UPMC hospital or affiliated health care provider or your current or future relationship with a health care insurance provider.

If you are eligible to participate, you will not be removed from this study without your consent.

\section{HOW CAN I GET MORE INFORMATION ABOUT THIS STUDY?}

If you have any further questions about this research study, you may contact Ms. Belcher (412-624-8938 orLAMPstudy@pitt.edu) or the investigators listed at the beginning of this consent form. If you have any questions about your rights as a research subject, please contact the Human Subjects Protection Advocate at the University of Pittsburgh IRB Office at 866-212-2668.

\section{Agreement to Participate}

By clicking "Yes, I agree," in the online survey, you are providing your consent to participate in this research study and your agreement with the following information:

- I have read the consent form for this study and any questions I had, including explanation of all terminology, have been answered to my satisfaction. A copy of this consent form has been made available to me.

- I understand that I am encouraged to ask questions about any aspect of this research study and that those questions will be answered by the researchers listed on the first page of this form.

- I understand that my participation in this study is voluntary and that I am free to refuse to participate or to withdraw my consent and discontinue my participation in this study at any time without affecting my future relationship with this institution.

- I consent to participate in this research study and provide my authorization to share my medical records with the research team for the purposes described above.

Completion of the following questions in the online survey will serve as verification of electronic consent to participate in this research study and HIPAA authorization for use of medical records for the purposes described above:

- Subject's full name

- Subject's birthdate

- Subject's answer to one of the following verifiable questions:

1. What is your mother's maiden name?

2. In what city were you born?

3. What high school did you attend?

Page 5 of 5 


\section{University of Pittsburgh \\ School of Nursing \\ Department of Health and Community Systems}

CONSENT TO ACT AS A PARTICIPANT IN A RESEARCH STUDY

TITLE OF RESEARCH PROJECT:

Characterizing Psychobehavioral Risks in Survivors of Multiple Primary Cancers: The LAMP Study

PRINCIPAL INVESTIGATOR: $\quad$ Sarah M. Belcher, PhD(c), RN, OCN ${ }^{\circledR}$

Doctoral Candidate

University of Pittsburgh School of Nursing

415 Victoria Building

3500 Victoria Street

Pittsburgh, PA 15261

412-624-8938

\section{CO-INVESTIGATORS:}

Leonard Appleman, MD, PhD

Physican, Division of Hematology-Oncology

UPMC

412-648-6507

Adam Brufsky, MD, PhD

Associate Chief, Division of Hematology-Oncology

Co-Director, Comprehensive Breast Center

UPMC

412-641-6500

Edward Chu, MD

Chief, Division of Hematology-Oncology

UPMC

412-648-6589

Robert Edwards, MD

Chair, Gynecology \& Reproductive Sciences

Magee-Womens Hospital of UPMC

412-641-4212

John Kirkwood, MD

Director, Melanoma and Skin Cancer Program

UPMC

412-623-7707
Dana Bovbjerg, PhD

Director, Biobehavioral Oncology Program University of Pittsburgh Cancer Institute 412-623-5965

Grace Campbell, PhD, MSW, RN, CRRN

Assistant Professor

School of Nursing

412-417-8804

Heidi Donovan, PhD, RN

Professor and Vice Chair for Research

School of Nursing

412-624-2699

Jonas Johnson, MD

Chairman, Otolaryngology

UPMC

412-647-2100

Susan Sereika, PhD

Director, Center for Research and Evaluation School of Nursing

412-624-0799 
Paula Sherwood, PhD, RN, CNRN, FAAN

Professor

School of Nursing

412-624-4802
Liza Villaruz, MD

Physician, Division of Hematology-Oncology

UPMC

412-648-6578

SOURCES OF SUPPORT: American Cancer Society Doctoral Degree Scholarship in Cancer Nursing Robert Wood Johnson Foundation Future of Nursing Scholars Nightingale Awards of Pennsylvania

University of Pittsburgh School of Nursing

You are being asked to take part in a research study being conducted by the University of Pittsburgh's School of Nursing. We will ask you to complete a one-time set of questionnaires that will take approximately 30-40 minutes to complete.

\section{WHY IS THIS RESEARCH BEING DONE?}

We are doing this study to help us understand the experiences of adult cancer survivors like you who have had two separate types of cancer. We call two or more separate types of cancers "multiple primary cancers." We want to learn more about how having multiple primary cancers impacts stress, health behaviors, and emotional and overall health.

\section{WHO IS BEING ASKED TO TAKE PART IN THIS RESEARCH STUDY?}

You are being invited to take part in this research study because you have a history of two separate types of cancer, or multiple primary cancers. We will enroll 450 multiple primary cancer survivors like you in this study.

\section{WHAT PROCEDURES WILL BE PERFORMED FOR RESEARCH PURPOSES?}

The study procedures consist of a one-time completion of a set of questionnaires and a medical record review. We will send you either a personalized internet link to complete the questionnaire online or a pencil-paper copy of the questionnaires with a pre-stamped, pre-addressed return envelope.

1. The questionnaire can be completed online, or we can mail you the questionnaire to do on paper. You will complete a set of questionnaires that include demographic and health information and questions about stress, health behaviors, your experience with cancer, and emotional and overall health.

If done on paper, you will then return the signed consent form (required) along with the completed questionnaires in the return envelope provided to you.

Online responses are automatically recorded.

We estimate that the questionnaire will take 30-40 minutes to complete.

Page 2 of 6 
If we do not receive your questionnaires after we sent you the initial packet in the mail, we will send you a reminder postcard two weeks after the initial letter and a reminder letter and replacement questionnaire three weeks after the initial letter.

2. We will review your medical record to find out about your health.

We will record information about types of cancer, dates diagnosed, cancer treatments, and any other illnesses you may have had.

3. A refusal form is requested if you do not want to participate in the study.

If you choose not to participate in the study, you will be asked to complete and return five questions to help us understand why people are not participating in this study. Answering these additional five questions is voluntary.

\section{WHAT ARE THE POSSIBLE RISKS, SIDE EFFECTS, AND DISCOMFORTS OF THIS RESEARCH STUDY?}

This is a very low risk study, but you should be aware of risks.

1. One potential risk is a breach of confidentiality, but we will do everything possible to protect your privacy. To protect your privacy, only Ms. Belcher (the principle investigator) and members of the research team will be aware of your participation in this research study. Your name will not be included on the questionnaires we ask you to complete or the information we collect from your medical record. Mailed questionnaires and information collected about you from your medical record will be kept in secure, locked file cabinets at the School of Nursing. All information will be identified only by a study ID number. The information linking these ID numbers with your identify will be kept separate from the research records and will be stored under lock and key. If you complete the questionnaires online, the website where you complete the questionnaire is secure, and your data will be safely stored and can only be accessed by study team members. All researchers involved in this study have been thoroughly trained to maintain your privacy. All information you provide will be kept by the Principle Investigator in a locked file cabinet within a locked office at the School of Nursing. Your identity will not be revealed in any description or publications of this research, and data will only be presented about groups and not individual participants.

2. Another possible risk of this research study may include stress from having to complete the questionnaires. If the questions cause you stress or discomfort, you can take a break from completing the questionnaires. If any individual questions makes you feel distressed (anxious, sad, or nervous), you do not have to answer them.

\section{WHAT ARE POSSIBLE BENEFITS FROM TAKING PART IN THIS STUDY?}

You will likely receive no direct benefit from taking part in this research study. The results from this study may benefit survivors like you who experience multiple primary cancer diagnoses in the future but will have no direct benefit to you.

\section{WILL I BE PAID IF I TAKE PART IN THIS RESEARCH STUDY?}

Each participant will be provided with a $\$ 5$ Amazon.com gift card code in recognition of his or her time and expertise after completing and returning the survey to Ms. Belcher.

Page 3 of 6 


\section{WHO WILL KNOW ABOUT MY PARTICIPATION IN THIS RESEARCH STUDY?}

Any information about you obtained from this research will be kept strictly confidential (private), and any data that includes your identity will be stored in locked files in Research Project Office or in a password protected computer system. All records related to your involvement in this research study will be stored in a locked file cabinet in a locked room at the School of Nursing. Your identity on these records, and in the corresponding data entered into the computerized system, will be indicated by an ID number rather than by your name. The information linking these ID numbers with your identity will be kept separate from the research records. You will not be identified by name in any publication of the research. All records will be retained by us for a minimum of seven years.

It is possible that we may use the information obtained from this study to answer more research questions in other research studies. This information may also be shared with other researchers here, and at other research centers, but those researchers will never be provided with any personal identifiers that would allow them to learn who you are.

\section{Why is my authorization being requested?}

We are also requesting your authorization, or permission, to review your medical records to confirm information about your cancer and medical history and treatments. The authorization to access your medical records will be valid for a minimum of 7 years. We will obtain the following information: your diagnoses and treatments, age, past medical history, and results of any tissue biopsies or blood tests done as part of your standard evaluation at the Cancer Center. This identifiable medical record information will be made available to members of the research team for an indefinite period of time. Your medical information, as well as information obtained during this research study, may be shared with other groups, possibly including authorized officials from the University of Pittsburgh Research Conduct and Compliance Office, for the purpose of monitoring the study. Authorized representatives of UPMC or affiliated health care providers may also have access to this information to provide services and address billing and operational issues.

We will make every attempt to protect your privacy and the confidentiality of your records, as described in this document, but cannot guarantee the confidentiality of your research records, including information obtained from your medical records once your personal information is disclosed to others outside UPMC or the University. You can always withdraw your authorization to allow the research team to review your medical records by contacting the investigator listed on the first page and making the request in writing. If you do so, you will no longer be permitted to participate in this study. Any information obtained from you up to that point will continue to be used by the research team.

\section{IS MY PARTICIPATION IN THIS STUDY VOLUNTARY?}

Your participation in this study is completely voluntary. You may refuse to take part in it, or you may stop participating at any time, even after signing this form. Your decision will not affect your relationship or current or future care at a UPMC hospital or affiliated health care provider or your current or future relationship with a health care insurance provider.

If you are eligible to participate, you will not be removed from this study without your consent.

Page 4 of 6 


\section{HOW CAN I GET MORE INFORMATION ABOUT THIS STUDY?}

If you have any further questions about this research study, you may contact Ms. Belcher (412-624-8938 orLAMPstudy@pitt.edu) or the investigators listed at the beginning of this consent form. If you have any questions about your rights as a research subject, please contact the Human Subjects Protection Advocate at the University of Pittsburgh IRB Office at 866-212-2668.

\section{Agreement to Participate}

- I have read the consent form for this study and any questions I had, including explanation of all terminology, have been answered to my satisfaction. A copy of this consent form has been provided to me.

- I understand that I am encouraged to ask questions about any aspect of this research study and that those questions will be answered by the researchers listed on the first page of this form.

- I understand that my participation in this study is voluntary and that I am free to refuse to participate or to withdraw my consent and discontinue my participation in this study at any time without affecting my future relationship with this institution.

- By signing this form, I consent to participate in this research study and provide my authorization to share my medical records with the research team.

\footnotetext{
Subject's Printed Name
} 


\section{Certification of Informed Consent}

\section{To Be Completed by Research Team}

I certify that the above-named individual(s) has been provided with information about the nature and purpose of this research study, and he/she has been informed of the potential benefits and possible risks of study participation. Contact information and encouragement to contact study personnel in case of any questions has been provided. We will always be available to address future questions as they arise. I further certify that no research component of this protocol was begun until after this consent form was signed.

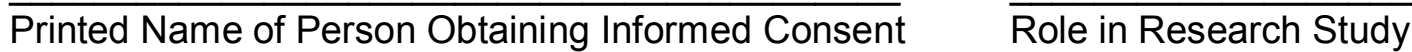


APPENDIX D

\section{HUMAN SUBJECTS TRAINING}




\section{COLLABORATIVE INSTITUTIONAL TRAINING INITIATIVE (CITI PROGRAM) COMPLETION REPORT - PART 1 OF 2 COURSEWORK REQUIREMENTS*}

* NOTE: Scores on this Requirements Report reflect quiz completions at the time all requirements for the course were met. See list below for details. See separate Transcript Report for more recent quiz scores, including those on optional (supplemental) course elements.

- Name:

- Institution Affiliation:

- Institution Email:

- Institution Unit:

- Phone:

- Curriculum Group:

- Course Learner Group:

- Stage:

- Description:

- Record ID:

- Completion Date:

- Expiration Date:

- Minimum Passing:

- Reported Score*:

Sarah Belcher (ID: 4013572)

University of Pittsburgh (ID: 2074)

smb208@pitt.edu

School of Nursing

412-624-2469

Biomedical Human Subjects Research

Biomedical Course

Stage 1 - Basic Course

Choose this group to satisfy CITI training requirements for Investigators and staff involved primarily in biomedical research with human subjects.

12328541

09-Feb-2014

09-Feb-2018

80

98

\section{REQUIRED AND ELECTIVE MODULES ONLY}

University of Pittsburgh (ID: 14517)

Belmont Report and Its Principles (ID: 1127)

History and Ethics of Human Subjects Research (ID: 498)

Basic Institutional Review Board (IRB) Regulations and Review Process (ID: 2)

Informed Consent (ID: 3)

Genetic Research in Human Populations (ID: 6)

Research With Protected Populations - Vulnerable Subjects: An Overview (ID: 7)

Research Involving Children (ID: 9)

Conflicts of Interest in Research Involving Human Subjects (ID: 488)

Research Involving Prisoners (ID: 8)

Stem Cell Research Oversight (Part I) (ID: 13882)

$\begin{array}{ll}\text { DATE COMPLETED } & \text { SCORE } \\ \text { 09-Feb-2014 } & \text { No Quiz } \\ \text { 09-Feb-2014 } & 3 / 3(100 \%) \\ 09-F e b-2014 & 7 / 7(100 \%) \\ 09-F e b-2014 & 5 / 5(100 \%) \\ 09-F e b-2014 & 4 / 4(100 \%) \\ 09-F e b-2014 & 2 / 2(100 \%) \\ 09-F e b-2014 & 4 / 4(100 \%) \\ 09-F e b-2014 & 3 / 3(100 \%) \\ 09-F e b-2014 & 5 / 5(100 \%) \\ 09-F e b-2014 & 3 / 4(75 \%) \\ 09-F e b-2014 & 5 / 5(100 \%)\end{array}$

For this Report to be valid, the learner identified above must have had a valid affiliation with the CITI Program subscribing institution identified above or have been a paid Independent Learner.

Verify at: www.citiprogram.org/verify/?ka5adbd32-73e2-4a18-96be-b270fdd39ffa-12328541

Collaborative Institutional Training Initiative (CITI Program)

Email: support@citiprogram.org

Phone: 888-529-5929

Web: https://www.citiprogram.org 


\section{COLLABORATIVE INSTITUTIONAL TRAINING INITIATIVE (CITI PROGRAM) COMPLETION REPORT - PART 2 OF 2 COURSEWORK TRANSCRIPT**}

${ }^{* *}$ NOTE: Scores on this Transcript Report reflect the most current quiz completions, including quizzes on optional (supplemental) elements of the course. See list below for details. See separate Requirements Report for the reported scores at the time all requirements for the course were met.

- Name: $\quad$ Sarah Belcher (ID: 4013572)

- Institution Affiliation: University of Pittsburgh (ID: 2074)

- Institution Email: $\quad$ smb208@pitt.edu

- Institution Unit: School of Nursing

- Phone: 412-624-2469

- Curriculum Group: Biomedical Human Subjects Research

- Course Learner Group: Biomedical Course

- Stage:

- Description:

Stage 1 - Basic Course

Choose this group to satisfy CITI training requirements for Investigators and staff involved primarily in biomedical research with human subjects.

- Record ID:

12328541

- Report Date:

07-Jun-2018

- Current Score**:

98

REQUIRED, ELECTIVE, AND SUPPLEMENTAL MODULES

History and Ethics of Human Subjects Research (ID: 498)

University of Pittsburgh (ID: 14517)

Informed Consent (ID: 3)

Belmont Report and Its Principles (ID: 1127)

Genetic Research in Human Populations (ID: 6)

Research Involving Prisoners (ID: 8)

Research Involving Children (ID: 9)

Conflicts of Interest in Research Involving Human Subjects (ID: 488)

Basic Institutional Review Board (IRB) Regulations and Review Process (ID: 2)

Stem Cell Research Oversight (Part I) (ID: 13882)

Research With Protected Populations - Vulnerable Subjects: An Overview (ID: 7)

$\begin{array}{ll}\text { MOST RECENT } & \text { SCORE } \\ \text { 09-Feb-2014 } & 7 / 7(100 \%) \\ \text { 09-Feb-2014 } & \text { No Quiz } \\ \text { 09-Feb-2014 } & 4 / 4(100 \%) \\ \text { 09-Feb-2014 } & 3 / 3(100 \%) \\ \text { 09-Feb-2014 } & 2 / 2(100 \%) \\ \text { 09-Feb-2014 } & 3 / 4(75 \%) \\ \text { 09-Feb-2014 } & 3 / 3(100 \%) \\ \text { 09-Feb-2014 } & 5 / 5(100 \%) \\ \text { 09-Feb-2014 } & 5 / 5(100 \%) \\ \text { 09-Feb-2014 } & 5 / 5(100 \%) \\ 09-F e b-2014 & 4 / 4(100 \%)\end{array}$

For this Report to be valid, the learner identified above must have had a valid affiliation with the CITI Program subscribing institution identified above or have been a paid Independent Learner.

Verify at: www.citiprogram.org/verify/?ka5adbd32-73e2-4a18-96be-b270fdd39ffa-12328541

Collaborative Institutional Training Initiative (CITI Program)

Email: support@citiprogram.org

Phone: 888-529-5929

Web: https://www.citiprogram.org 


\section{COLLABORATIVE INSTITUTIONAL TRAINING INITIATIVE (CITI PROGRAM) COMPLETION REPORT - PART 1 OF 2 COURSEWORK REQUIREMENTS*}

* NOTE: Scores on this Requirements Report reflect quiz completions at the time all requirements for the course were met. See list below for details. See separate Transcript Report for more recent quiz scores, including those on optional (supplemental) course elements.

- Name:

- Institution Affiliation:

- Institution Email:

- Institution Unit:

- Phone:

- Curriculum Group:

- Course Learner Group:

- Stage:

- Description:

- Record ID:

- Completion Date:

- Expiration Date:

- Minimum Passing:

- Reported Score*:

Sarah Belcher (ID: 4013572)

University of Pittsburgh (ID: 2074)

smb208@pitt.edu

School of Nursing

412-624-2469

Biomedical Responsible Conduct of Research

Same as Curriculum Group

Stage 1 - RCR

This course is for investigators, staff and students with an interest or focus in Biomedical Research. This course contains text, embedded case studies AND quizzes.

12328543

09-Feb-2014

09-Feb-2018

80

97

\section{REQUIRED AND ELECTIVE MODULES ONLY}

Introduction to the Responsible Conduct of Research Archived 1248 (ID: 1248)

Research Misconduct (RCR-Biomed) (ID: 1215)

Data Management (RCR-Biomed) (ID: 1308)

Authorship (RCR-Biomed) (ID: 1380)

Mentoring (RCR-Interdisciplinary) (ID: 1250)

Collaborative Research (RCR-Biomed) (ID: 1450)

Responsible Conduct of Research (RCR) Course Conclusion (ID: 1043)

Conflicts of Interest (RCR-Biomed) (ID: 1622)

$\begin{array}{ll}\text { DATE COMPLETED } & \text { SCORE } \\ \text { 09-Feb-2014 } & \text { No Quiz } \\ \text { 09-Feb-2014 } & 5 / 5(100 \%) \\ \text { 09-Feb-2014 } & 5 / 5(100 \%) \\ \text { 09-Feb-2014 } & 5 / 5(100 \%) \\ 09-F e b-2014 & 5 / 5(100 \%) \\ \text { 09-Feb-2014 } & 5 / 5(100 \%) \\ \text { 09-Feb-2014 } & \text { No Quiz } \\ \text { 09-Feb-2014 } & 5 / 6(83 \%)\end{array}$

For this Report to be valid, the learner identified above must have had a valid affiliation with the CITI Program subscribing institution identified above or have been a paid Independent Learner.

Verify at: $\underline{w w w . c i t i p r o g r a m . o r g / v e r i f y / ? k 72260891-402 f-4 e d a-88 f 0-789 a 6 e 6 e 5 e 86-12328543 ~}$

Collaborative Institutional Training Initiative (CITI Program)

Email: support@citiprogram.org

Phone: 888-529-5929

Web: https://www.citiprogram.org 


\section{COLLABORATIVE INSTITUTIONAL TRAINING INITIATIVE (CITI PROGRAM) COMPLETION REPORT - PART 2 OF 2 COURSEWORK TRANSCRIPT**}

** NOTE: Scores on this Transcript Report reflect the most current quiz completions, including quizzes on optional (supplemental) elements of the course. See list below for details. See separate Requirements Report for the reported scores at the time all requirements for the course were met.

- Name: $\quad$ Sarah Belcher (ID: 4013572)

- Institution Affiliation: University of Pittsburgh (ID: 2074)

- Institution Email: $\quad$ smb208@pitt.edu

- Institution Unit: School of Nursing

- Phone: 412-624-2469

- Curriculum Group: Biomedical Responsible Conduct of Research

- Course Learner Group: Same as Curriculum Group

- Stage: Stage 1 - RCR

- Description: This course is for investigators, staff and students with an interest or focus in Biomedical Research. This course contains text, embedded case studies AND quizzes.

- Record ID:

12328543

- Report Date:

07-Jun-2018

- Current Score**:

97

\section{REQUIRED, ELECTIVE, AND SUPPLEMENTAL MODULES}

Mentoring (RCR-Interdisciplinary) (ID: 1250)

Data Management (RCR-Biomed) (ID: 1308)

Authorship (RCR-Biomed) (ID: 1380)

Collaborative Research (RCR-Biomed) (ID: 1450)

Conflicts of Interest (RCR-Biomed) (ID: 1622)

Plagiarism (RCR-Basic) (ID: 15156)

Authorship (RCR-Basic) (ID: 16597)

Research Misconduct (RCR-Biomed) (ID: 1215)

Introduction to the Responsible Conduct of Research Archived 1248 (ID: 1248)

Collaborative Research (RCR-Basic) (ID: 16598)

Data Management (RCR-Basic) (ID: 16600)

Mentoring (RCR-Basic) (ID: 16602)

Peer Review (RCR-Basic) (ID: 16603)

Research Misconduct (RCR-Basic) (ID: 16604)

Responsible Conduct of Research (RCR) Course Conclusion (ID: 1043)

$\begin{array}{ll}\text { MOST RECENT } & \text { SCORE } \\ \text { 09-Feb-2014 } & 5 / 5(100 \%) \\ \text { 09-Feb-2014 } & 5 / 5(100 \%) \\ \text { 09-Feb-2014 } & 5 / 5(100 \%) \\ \text { 09-Feb-2014 } & 5 / 5(100 \%) \\ \text { 09-Feb-2014 } & 5 / 6(83 \%) \\ \text { 02-Mar-2018 } & 5 / 5(100 \%) \\ \text { 02-Mar-2018 } & 4 / 5(80 \%) \\ \text { 09-Feb-2014 } & 5 / 5(100 \%) \\ \text { 09-Feb-2014 } & \text { No Quiz } \\ \text { 02-Mar-2018 } & 5 / 5(100 \%) \\ \text { 02-Mar-2018 } & 5 / 5(100 \%) \\ \text { 02-Mar-2018 } & 5 / 5(100 \%) \\ \text { 02-Mar-2018 } & 5 / 5(100 \%) \\ \text { 02-Mar-2018 } & 5 / 5(100 \%) \\ \text { 09-Feb-2014 } & \text { No Quiz }\end{array}$

For this Report to be valid, the learner identified above must have had a valid affiliation with the CITI Program subscribing institution identified above or have been a paid Independent Learner.

Verify at: www.citiprogram.org/verify/?k72260891-402f-4eda-88f0-789a6e6e5e86-12328543

Collaborative Institutional Training Initiative (CITI Program)

Email: support@citiprogram.org

Phone: 888-529-5929

Web: https://www.citiprogram.org 


\section{COLLABORATIVE INSTITUTIONAL TRAINING INITIATIVE (CITI PROGRAM) COMPLETION REPORT - PART 1 OF 2 COURSEWORK REQUIREMENTS*}

* NOTE: Scores on this Requirements Report reflect quiz completions at the time all requirements for the course were met. See list below for details. See separate Transcript Report for more recent quiz scores, including those on optional (supplemental) course elements.

- Name:

- Institution Affiliation:

- Institution Email:

- Institution Unit:

- Phone:

- Curriculum Group:

- Course Learner Group: Cor

- Stage:

- Record ID:

- Completion Date:

- Expiration Date:

- Minimum Passing:

- Reported Score*:
Sarah Belcher (ID: 4013572)

University of Pittsburgh (ID: 2074)

smb208@pitt.edu

School of Nursing

412-624-2469

CITI Conflicts of Interest

Conflicts of Interest

Stage 1 - Basic Course

14006386

11-Sep-2014

11-Sep-2018

80

80

\section{REQUIRED AND ELECTIVE MODULES ONLY}

CITI Conflict of Interest Course - Introduction (COI-Basic) (ID: 15177)

Financial Conflicts of Interest: Overview, Investigator Responsibilities, and COI Rules (COI-Basic) (ID: 15070) Institutional Responsibilities as They Affect Investigators (COI-Basic) (ID: 15072)

Conflicts of Interest Institution-Specific Policies (ID: 15179)

Conflicts of Commitment and Conscience (COI-Basic) (ID: 15073)
DATE COMPLETED SCORE

11-Sep-2014 No Quiz

11-Sep-2014 4/5 (80\%)

11-Sep-2014 3/5 $\quad 30 \%)$

11-Sep-2014 10/10 (100\%)

11-Sep-2014 $\quad 3 / 5(60 \%)$

For this Report to be valid, the learner identified above must have had a valid affiliation with the CITI Program subscribing institution identified above or have been a paid Independent Learner.

Verify at: www.citiprogram.org/verify/?kb4a7be0c-d537-4dd2-80cf-b18ff50a2c3f-14006386

Collaborative Institutional Training Initiative (CITI Program)

Email: support@citiprogram.org

Phone: 888-529-5929

Web: https://www.citiprogram.org 


\section{COLLABORATIVE INSTITUTIONAL TRAINING INITIATIVE (CITI PROGRAM) COMPLETION REPORT - PART 2 OF 2 COURSEWORK TRANSCRIPT**}

** NOTE: Scores on this Transcript Report reflect the most current quiz completions, including quizzes on optional (supplemental) elements of the course. See list below for details. See separate Requirements Report for the reported scores at the time all requirements for the course were met.

- Name: $\quad$ Sarah Belcher (ID: 4013572)

- Institution Affiliation: University of Pittsburgh (ID: 2074)

- Institution Email: $\quad$ smb208@pitt.edu

- Institution Unit: School of Nursing

- Phone: 412-624-2469

- Curriculum Group: CITI Conflicts of Interest

- Course Learner Group: Conflicts of Interest

- Stage: Stage 1 - Basic Course

- Record ID: 14006386

- Report Date: 07-Jun-2018

- Current Score ${ }^{* *}$ : 80

\section{REQUIRED, ELECTIVE, AND SUPPLEMENTAL MODULES}

CITI Conflict of Interest Course - Introduction (COI-Basic) (ID: 15177)

Conflicts of Interest Institution-Specific Policies (ID: 15179)

Financial Conflicts of Interest: Overview, Investigator Responsibilities, and COI Rules (COI-Basic) (ID: 15070)

Institutional Responsibilities as They Affect Investigators (COI-Basic) (ID: 15072)

Conflicts of Commitment and Conscience (COI-Basic) (ID: 15073)
MOST RECENT SCORE

11-Sep-2014 No Quiz

11-Sep-2014 10/10 (100\%)

11-Sep-2014 4/5 (80\%)

11-Sep-2014 3/5 (60\%)

11-Sep-2014 3/5 (60\%)

For this Report to be valid, the learner identified above must have had a valid affiliation with the CITI Program subscribing institution identified above or have been a paid Independent Learner.

Verify at: www.citiprogram.org/verify/?kb4a7be0c-d537-4dd2-80cf-b18ff50a2c3f-14006386

Collaborative Institutional Training Initiative (CITI Program)

Email: support@citiprogram.org

Phone: 888-529-5929

Web: https://www.citiprogram.org 


\section{COLLABORATIVE INSTITUTIONAL TRAINING INITIATIVE (CITI PROGRAM) COMPLETION REPORT - PART 1 OF 2 COURSEWORK REQUIREMENTS*}

* NOTE: Scores on this Requirements Report reflect quiz completions at the time all requirements for the course were met. See list below for details. See separate Transcript Report for more recent quiz scores, including those on optional (supplemental) course elements.

- Name:

- Institution Affiliation:

- Institution Email:

- Institution Unit:

- Phone:

- Curriculum Group:

- Course Learner Grou

- Stage:

- Record ID:

- Completion Date:

- Expiration Date:

- Minimum Passing:

- Reported Score*:
Sarah Belcher (ID: 4013572)

University of Pittsburgh (ID: 2074)

smb208@pitt.edu

School of Nursing

412-624-2469

Information Privacy \& Security

Privacy \& Information Security

Stage 1 - Basic Course

\section{4}

03-Jul-2017

03-Jul-2021

80

95

\section{REQUIRED AND ELECTIVE MODULES ONLY}

Basics of Health Privacy (ID: 1417)

Health Privacy Issues for Researchers (ID: 1419)

Basics of Information Security, Part 1 (ID: 1423)

Basics of Information Security, Part 2 (ID: 1424)

$\begin{array}{ll}\text { DATE COMPLETED } & \text { SCORE } \\ \text { 03-Jul-2017 } & 5 / 5(100 \%) \\ \text { 03-Jul-2017 } & 5 / 5(100 \%) \\ \text { 03-Jul-2017 } & 4 / 5(80 \%) \\ \text { 03-Jul-2017 } & 5 / 5(100 \%)\end{array}$

For this Report to be valid, the learner identified above must have had a valid affiliation with the CITI Program subscribing institution identified above or have been a paid Independent Learner.

Verify at: www.citiprogram.org/verify/?kf0084fa7-f6c2-44c0-95c4-20f8ab361f30-23759774

Collaborative Institutional Training Initiative (CITI Program)

Email: support@citiprogram.org

Phone: 888-529-5929

Web: https://www.citiprogram.org 


\section{COLLABORATIVE INSTITUTIONAL TRAINING INITIATIVE (CITI PROGRAM) COMPLETION REPORT - PART 2 OF 2 COURSEWORK TRANSCRIPT**}

** NOTE: Scores on this Transcript Report reflect the most current quiz completions, including quizzes on optional (supplemental) elements of the course. See list below for details. See separate Requirements Report for the reported scores at the time all requirements for the course were met.

- Name: $\quad$ Sarah Belcher (ID: 4013572)

- Institution Affiliation: University of Pittsburgh (ID: 2074)

- Institution Email: $\quad$ smb208@pitt.edu

- Institution Unit: School of Nursing

- Phone: 412-624-2469

- Curriculum Group: Information Privacy \& Security

- Course Learner Group: Privacy \& Information Security

- Stage: Stage 1 - Basic Course

- Record ID: 23759774

- Report Date: 07-Jun-2018

- Current Score**: 95

\section{REQUIRED, ELECTIVE, AND SUPPLEMENTAL MODULES}

Basics of Health Privacy (ID: 1417)

Health Privacy Issues for Researchers (ID: 1419)

Basics of Information Security, Part 1 (ID: 1423)

Basics of Information Security, Part 2 (ID: 1424)

\section{MOST RECENT}

03-Jul-2017

03-Jul-2017

03-Jul-2017

03-Jul-2017
SCORE

$5 / 5(100 \%)$

$5 / 5(100 \%)$

$4 / 5(80 \%)$

$5 / 5(100 \%)$

For this Report to be valid, the learner identified above must have had a valid affiliation with the CITI Program subscribing institution identified above or have been a paid Independent Learner.

Verify at: www.citiprogram.org/verify/?kf0084fa7-f6c2-44c0-95c4-20f8ab361f30-23759774

Collaborative Institutional Training Initiative (CITI Program)

Email: support@citiprogram.org

Phone: 888-529-5929

Web: https://www.citiprogram.org 


\section{COLLABORATIVE INSTITUTIONAL TRAINING INITIATIVE (CITI PROGRAM) COMPLETION REPORT - PART 1 OF 2 COURSEWORK REQUIREMENTS*}

* NOTE: Scores on this Requirements Report reflect quiz completions at the time all requirements for the course were met. See list below for details. See separate Transcript Report for more recent quiz scores, including those on optional (supplemental) course elements.

- Name:

- Institution Affiliation:

- Institution Email:

- Institution Unit:

- Phone:

- Curriculum Group:

- Course Learner Group:

- Stage:

- Record ID:

- Completion Date:

- Expiration Date:

- Minimum Passing:

- Reported Score*:
Sarah Belcher (ID: 4013572)

University of Pittsburgh (ID: 2074)

smb208@pitt.edu

School of Nursing

412-624-2469

Responsible Conduct of Research

Same as Curriculum Group

Stage 1 - Basic Course

\author{
26362482 \\ 02-Mar-2018 \\ 01-Mar-2022 \\ 80 \\ 97
}

\section{REQUIRED AND ELECTIVE MODULES ONLY}

Authorship (RCR-Basic) (ID: 16597)

Collaborative Research (RCR-Basic) (ID: 16598)

Data Management (RCR-Basic) (ID: 16600)

Mentoring (RCR-Basic) (ID: 16602)

Peer Review (RCR-Basic) (ID: 16603)

Research Misconduct (RCR-Basic) (ID: 16604)

Plagiarism (RCR-Basic) (ID: 15156)

$\begin{array}{ll}\text { DATE COMPLETED } & \text { SCORE } \\ \text { 02-Mar-2018 } & 4 / 5(80 \%) \\ \text { 02-Mar-2018 } & 5 / 5(100 \%) \\ \text { 02-Mar-2018 } & 5 / 5(100 \%) \\ \text { 02-Mar-2018 } & 5 / 5(100 \%) \\ \text { 02-Mar-2018 } & 5 / 5(100 \%) \\ \text { 02-Mar-2018 } & 5 / 5(100 \%) \\ \text { 02-Mar-2018 } & 5 / 5(100 \%)\end{array}$

For this Report to be valid, the learner identified above must have had a valid affiliation with the CITI Program subscribing institution identified above or have been a paid Independent Learner.

Verify at: www.citiprogram.org/verify/?kaafd1274-d1eb-4f06-ad49-07dc88e728af-26362482

Collaborative Institutional Training Initiative (CITI Program)

Email: support@citiprogram.org

Phone: 888-529-5929

Web: https://www.citiprogram.org 


\section{COLLABORATIVE INSTITUTIONAL TRAINING INITIATIVE (CITI PROGRAM) COMPLETION REPORT - PART 2 OF 2 COURSEWORK TRANSCRIPT**}

** NOTE: Scores on this Transcript Report reflect the most current quiz completions, including quizzes on optional (supplemental) elements of the course. See list below for details. See separate Requirements Report for the reported scores at the time all requirements for the course were met.

- Name: $\quad$ Sarah Belcher (ID: 4013572)

- Institution Affiliation: University of Pittsburgh (ID: 2074)

- Institution Email: $\quad$ smb208@pitt.edu

- Institution Unit: School of Nursing

- Phone: 412-624-2469

- Curriculum Group: Responsible Conduct of Research

- Course Learner Group: Same as Curriculum Group

- Stage: Stage 1 - Basic Course

- Record ID: 26362482

- Report Date: 07-Jun-2018

- Current Score**: 97

\section{REQUIRED, ELECTIVE, AND SUPPLEMENTAL MODULES}

Plagiarism (RCR-Basic) (ID: 15156)

Authorship (RCR-Basic) (ID: 16597)

Collaborative Research (RCR-Basic) (ID: 16598)

Data Management (RCR-Basic) (ID: 16600)

Mentoring (RCR-Basic) (ID: 16602)

Peer Review (RCR-Basic) (ID: 16603)

Research Misconduct (RCR-Basic) (ID: 16604)

$\begin{array}{ll}\text { MOST RECENT } & \text { SCORE } \\ \text { 02-Mar-2018 } & 5 / 5(100 \%) \\ \text { 02-Mar-2018 } & 4 / 5(80 \%) \\ \text { 02-Mar-2018 } & 5 / 5(100 \%) \\ \text { 02-Mar-2018 } & 5 / 5(100 \%) \\ \text { 02-Mar-2018 } & 5 / 5(100 \%) \\ \text { 02-Mar-2018 } & 5 / 5(100 \%) \\ \text { 02-Mar-2018 } & 5 / 5(100 \%)\end{array}$

For this Report to be valid, the learner identified above must have had a valid affiliation with the CITI Program subscribing institution identified above or have been a paid Independent Learner.

Verify at: www.citiprogram.org/verify/?kaafd1274-d1eb-4f06-ad49-07dc88e728af-26362482

Collaborative Institutional Training Initiative (CITI Program)

Email: support@citiprogram.org

Phone: 888-529-5929

Web: https://www.citiprogram.org 


\section{COLLABORATIVE INSTITUTIONAL TRAINING INITIATIVE (CITI PROGRAM) COMPLETION REPORT - PART 1 OF 2 COURSEWORK REQUIREMENTS*}

* NOTE: Scores on this Requirements Report reflect quiz completions at the time all requirements for the course were met. See list below for details. See separate Transcript Report for more recent quiz scores, including those on optional (supplemental) course elements.

- Name:

- Institution Affiliation:

- Institution Email:

- Institution Unit:

- Phone:

- Curriculum Group:

- Course Learner Group:

- Stage:

- Description:

- Record ID:

- Completion Date:

- Expiration Date:

- Minimum Passing:

- Reported Score*:
Sarah Belcher (ID: 4013572)

University of Pittsburgh (ID: 2074)

smb208@pitt.edu

School of Nursing

412-624-2469

Social and Behavioral Science Human Subjects

Social-Behavioral-Educational Course

Stage 2 - Refresher Course

Choose this group to satisfy CITI training requirements for Investigators and staff involved primarily in Social/Behavioral Research with human subjects.

14006384

29-Jul-2016

29-Jul-2020

80

94

\section{REQUIRED AND ELECTIVE MODULES ONLY}

SBE Refresher 1 - Instructions (ID: 943)

SBE Refresher 1 - History and Ethical Principles (ID: 936)

SBE Refresher 1 - Federal Regulations for Protecting Research Subjects (ID: 937)

SBE Refresher 1 - Informed Consent (ID: 938)

SBE Refresher 1 - Defining Research with Human Subjects (ID: 15029)

SBE Refresher 1 - Assessing Risk (ID: 15034)

SBE Refresher 1 - Privacy and Confidentiality (ID: 15035)

SBE Refresher 1 - International Research (ID: 15028)

SBE Refresher 1 - Research in Educational Settings (ID: 940)

\section{DATE COMPLETED}

29-Jul-2016

29-Jul-2016

29-Jul-2016

29-Jul-2016

29-Jul-2016

29-Jul-2016

29-Jul-2016

29-Jul-2016

29-Jul-2016
SCORE

No Quiz $2 / 2(100 \%)$

$2 / 2(100 \%)$

$2 / 2(100 \%)$

$2 / 2(100 \%)$

$2 / 2(100 \%)$

$2 / 2(100 \%)$

$1 / 2(50 \%)$

$2 / 2(100 \%)$

For this Report to be valid, the learner identified above must have had a valid affiliation with the CITI Program subscribing institution identified above or have been a paid Independent Learner.

Verify at: www.citiprogram.org/verify/?k039bda8f-3133-4de8-8031-a3a8c22bae5e-14006384

Collaborative Institutional Training Initiative (CITI Program) Email: support@citiprogram.org

Phone: 888-529-5929

Web: https://www.citiprogram.org 


\section{COLLABORATIVE INSTITUTIONAL TRAINING INITIATIVE (CITI PROGRAM) COMPLETION REPORT - PART 2 OF 2 COURSEWORK TRANSCRIPT**}

** NOTE: Scores on this Transcript Report reflect the most current quiz completions, including quizzes on optional (supplemental) elements of the course. See list below for details. See separate Requirements Report for the reported scores at the time all requirements for the course were met.

- Name: $\quad$ Sarah Belcher (ID: 4013572)

- Institution Affiliation: University of Pittsburgh (ID: 2074)

- Institution Email: $\quad$ smb208@pitt.edu

- Institution Unit: School of Nursing

- Phone: 412-624-2469

- Curriculum Group: Social and Behavioral Science Human Subjects

- Course Learner Group: Social-Behavioral-Educational Course

- Stage: Stage 2 - Refresher Course

- Description: Choose this group to satisfy CITI training requirements for Investigators and staff involved primarily in Social/Behavioral Research with human subjects.

- Record ID:

14006384

- Report Date:

07-Jun-2018

- Current Score**:

94

\section{REQUIRED, ELECTIVE, AND SUPPLEMENTAL MODULES}

SBE Refresher 1 - History and Ethical Principles (ID: 936)

SBE Refresher 1 - Federal Regulations for Protecting Research Subjects (ID: 937)

SBE Refresher 1 - Informed Consent (ID: 938)

SBE Refresher 1 - Research in Educational Settings (ID: 940)

SBE Refresher 1 - Instructions (ID: 943)

SBE Refresher 1 - International Research (ID: 15028)

SBE Refresher 1 - Defining Research with Human Subjects (ID: 15029)

SBE Refresher 1 - Assessing Risk (ID: 15034)

SBE Refresher 1 - Privacy and Confidentiality (ID: 15035)

\section{MOST RECENT}

29-Jul-2016

29-Jul-2016

29-Jul-2016

29-Jul-2016

29-Jul-2016

29-Jul-2016

29-Jul-2016

29-Jul-2016

29-Jul-2016
SCORE

$2 / 2(100 \%)$

$2 / 2(100 \%)$

$2 / 2(100 \%)$

$2 / 2(100 \%)$

No Quiz

$1 / 2(50 \%)$

$2 / 2(100 \%)$

$2 / 2(100 \%)$

$2 / 2(100 \%)$

For this Report to be valid, the learner identified above must have had a valid affiliation with the CITI Program subscribing institution identified above or have been a paid Independent Learner.

Verify at: www.citiprogram.org/verify/?k039bda8f-3133-4de8-8031-a3a8c22bae5e-14006384

Collaborative Institutional Training Initiative (CITI Program)

Email: support@citiprogram.org

Phone: 888-529-5929

Web: https://www.citiprogram.org 
APPENDIX E

\section{PERMISSION TO USE PUBLISHED MANUSCRIPTS}




\section{JOHN WILEY AND SONS LICENSE TERMS AND CONDITIONS}

May 06, 2018

This Agreement between Ms. Sarah Belcher ("You") and John Wiley and Sons ("John Wiley and Sons") consists of your license details and the terms and conditions provided by John Wiley and Sons and Copyright Clearance Center.

License Number

License date

Licensed Content Publisher

Licensed Content Publication

Licensed Content Title

Licensed Content Author

Licensed Content Date

Licensed Content Volume

Licensed Content Issue

Licensed Content Pages

Type of use

Requestor type

Format

Portion

Will you be translating?

Title of your thesis /

dissertation

Expected completion date

Expected size (number of pages)

Requestor Location

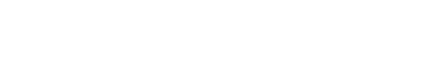

Publisher Tax ID

Total

Terms and Conditions
4343140680652

May 06, 2018

John Wiley and Sons

Psycho-Oncology

Examining the relationship between multiple primary cancers and psychological distress: A review of current literature

Sarah M. Belcher, Emilie A. Hausmann, Susan M. Cohen, et al

Nov 21, 2016

26

12

10

Dissertation/Thesis

Author of this Wiley article

Electronic

Full article

No

Characterizing Psychobehavioral Risks in Survivors of Multiple Primary Cancers

Jun 2018

100

Ms. Sarah Belcher 1720 Wightman St.

PITTSBURGH, PA 15217

United States

Attn: Ms. Sarah Belcher

EU826007151

0.00 USD 


\section{TERMS AND CONDITIONS}

This copyrighted material is owned by or exclusively licensed to John Wiley \& Sons, Inc. or one of its group companies (each a"Wiley Company") or handled on behalf of a society with which a Wiley Company has exclusive publishing rights in relation to a particular work (collectively "WILEY"). By clicking "accept" in connection with completing this licensing transaction, you agree that the following terms and conditions apply to this transaction (along with the billing and payment terms and conditions established by the Copyright Clearance Center Inc., ("CCC's Billing and Payment terms and conditions"), at the time that you opened your RightsLink account (these are available at any time at http://myaccount.copyright.com).

\section{Terms and Conditions}

- The materials you have requested permission to reproduce or reuse (the "Wiley Materials") are protected by copyright.

- You are hereby granted a personal, non-exclusive, non-sub licensable (on a standalone basis), non-transferable, worldwide, limited license to reproduce the Wiley Materials for the purpose specified in the licensing process. This license, and any CONTENT (PDF or image file) purchased as part of your order, is for a one-time use only and limited to any maximum distribution number specified in the license. The first instance of republication or reuse granted by this license must be completed within two years of the date of the grant of this license (although copies prepared before the end date may be distributed thereafter). The Wiley Materials shall not be used in any other manner or for any other purpose, beyond what is granted in the license. Permission is granted subject to an appropriate acknowledgement given to the author, title of the material/book/journal and the publisher. You shall also duplicate the copyright notice that appears in the Wiley publication in your use of the Wiley Material. Permission is also granted on the understanding that nowhere in the text is a previously published source acknowledged for all or part of this Wiley Material. Any third party content is expressly excluded from this permission.

-With respect to the Wiley Materials, all rights are reserved. Except as expressly granted by the terms of the license, no part of the Wiley Materials may be copied, modified, adapted (except for minor reformatting required by the new Publication), translated, reproduced, transferred or distributed, in any form or by any means, and no derivative works may be made based on the Wiley Materials without the prior permission of the respective copyright owner.For STM Signatory Publishers clearing permission under the terms of the STM Permissions Guidelines only, the terms of the license are extended to include subsequent editions and for editions in other languages, provided such editions are for the work as a whole in situ and does not involve the separate exploitation of the permitted figures or extracts, You may not alter, remove or suppress in any manner any copyright, trademark or other notices displayed by the Wiley Materials. You may not license, rent, sell, loan, lease, pledge, offer as security, transfer or assign the Wiley Materials on a stand-alone basis, or any of the rights granted to you hereunder to any other person. 
- The Wiley Materials and all of the intellectual property rights therein shall at all times remain the exclusive property of John Wiley \& Sons Inc, the Wiley Companies, or their respective licensors, and your interest therein is only that of having possession of and the right to reproduce the Wiley Materials pursuant to Section 2 herein during the continuance of this Agreement. You agree that you own no right, title or interest in or to the Wiley Materials or any of the intellectual property rights therein. You shall have no rights hereunder other than the license as provided for above in Section 2. No right, license or interest to any trademark, trade name, service mark or other branding ("Marks") of WILEY or its licensors is granted hereunder, and you agree that you shall not assert any such right, license or interest with respect thereto

- NEITHER WILEY NOR ITS LICENSORS MAKES ANY WARRANTY OR REPRESENTATION OF ANY KIND TO YOU OR ANY THIRD PARTY, EXPRESS, IMPLIED OR STATUTORY, WITH RESPECT TO THE MATERIALS OR THE ACCURACY OF ANY INFORMATION CONTAINED IN THE MATERIALS, INCLUDING, WITHOUT LIMITATION, ANY IMPLIED WARRANTY OF MERCHANTABILITY, ACCURACY, SATISFACTORY QUALITY, FITNESS FOR A PARTICULAR PURPOSE, USABILITY, INTEGRATION OR NON-INFRINGEMENT AND ALL SUCH WARRANTIES ARE HEREBY EXCLUDED BY WILEY AND ITS LICENSORS AND WAIVED BY YOU.

- WILEY shall have the right to terminate this Agreement immediately upon breach of this Agreement by you.

- You shall indemnify, defend and hold harmless WILEY, its Licensors and their respective directors, officers, agents and employees, from and against any actual or threatened claims, demands, causes of action or proceedings arising from any breach of this Agreement by you.

- IN NO EVENT SHALL WILEY OR ITS LICENSORS BE LIABLE TO YOU OR ANY OTHER PARTY OR ANY OTHER PERSON OR ENTITY FOR ANY SPECIAL, CONSEQUENTIAL, INCIDENTAL, INDIRECT, EXEMPLARY OR PUNITIVE DAMAGES, HOWEVER CAUSED, ARISING OUT OF OR IN CONNECTION WITH THE DOWNLOADING, PROVISIONING, VIEWING OR USE OF THE MATERIALS REGARDLESS OF THE FORM OF ACTION, WHETHER FOR BREACH OF CONTRACT, BREACH OF WARRANTY, TORT, NEGLIGENCE, INFRINGEMENT OR OTHERWISE (INCLUDING, WITHOUT LIMITATION, DAMAGES BASED ON LOSS OF PROFITS, DATA, FILES, USE, BUSINESS OPPORTUNITY OR CLAIMS OF THIRD PARTIES), AND WHETHER OR NOT THE PARTY HAS BEEN ADVISED OF THE POSSIBILITY OF SUCH DAMAGES. THIS LIMITATION SHALL APPLY NOTWITHSTANDING ANY FAILURE OF ESSENTIAL PURPOSE OF ANY LIMITED REMEDY PROVIDED HEREIN.

- Should any provision of this Agreement be held by a court of competent jurisdiction to be illegal, invalid, or unenforceable, that provision shall be deemed amended to 
achieve as nearly as possible the same economic effect as the original provision, and the legality, validity and enforceability of the remaining provisions of this Agreement shall not be affected or impaired thereby.

- The failure of either party to enforce any term or condition of this Agreement shall not constitute a waiver of either party's right to enforce each and every term and condition of this Agreement. No breach under this agreement shall be deemed waived or excused by either party unless such waiver or consent is in writing signed by the party granting such waiver or consent. The waiver by or consent of a party to a breach of any provision of this Agreement shall not operate or be construed as a waiver of or consent to any other or subsequent breach by such other party.

- This Agreement may not be assigned (including by operation of law or otherwise) by you without WILEY's prior written consent.

- Any fee required for this permission shall be non-refundable after thirty (30) days from receipt by the CCC.

- These terms and conditions together with CCC's Billing and Payment terms and conditions (which are incorporated herein) form the entire agreement between you and WILEY concerning this licensing transaction and (in the absence of fraud) supersedes all prior agreements and representations of the parties, oral or written. This Agreement may not be amended except in writing signed by both parties. This Agreement shall be binding upon and inure to the benefit of the parties' successors, legal representatives, and authorized assigns.

- In the event of any conflict between your obligations established by these terms and conditions and those established by CCC's Billing and Payment terms and conditions, these terms and conditions shall prevail.

- WILEY expressly reserves all rights not specifically granted in the combination of (i) the license details provided by you and accepted in the course of this licensing transaction, (ii) these terms and conditions and (iii) CCC's Billing and Payment terms and conditions.

- This Agreement will be void if the Type of Use, Format, Circulation, or Requestor Type was misrepresented during the licensing process.

- This Agreement shall be governed by and construed in accordance with the laws of the State of New York, USA, without regards to such state's conflict of law rules. Any legal action, suit or proceeding arising out of or relating to these Terms and Conditions or the breach thereof shall be instituted in a court of competent jurisdiction in New York County in the State of New York in the United States of America and each party hereby consents and submits to the personal jurisdiction of such court, waives any objection to venue in such court and consents to service of process by registered or certified mail, return receipt requested, at the last known address of such party. 


\section{WILEY OPEN ACCESS TERMS AND CONDITIONS}

Wiley Publishes Open Access Articles in fully Open Access Journals and in Subscription journals offering Online Open. Although most of the fully Open Access journals publish open access articles under the terms of the Creative Commons Attribution (CC BY) License only, the subscription journals and a few of the Open Access Journals offer a choice of Creative Commons Licenses. The license type is clearly identified on the article.

\section{The Creative Commons Attribution License}

The Creative Commons Attribution License (CC-BY) allows users to copy, distribute and transmit an article, adapt the article and make commercial use of the article. The CC-BY license permits commercial and non-

\section{Creative Commons Attribution Non-Commercial License}

The Creative Commons Attribution Non-Commercial (CC-BY-NC)License permits use, distribution and reproduction in any medium, provided the original work is properly cited and is not used for commercial purposes.(see below)

\section{Creative Commons Attribution-Non-Commercial-NoDerivs License}

The Creative Commons Attribution Non-Commercial-NoDerivs License (CC-BY-NC-ND) permits use, distribution and reproduction in any medium, provided the original work is properly cited, is not used for commercial purposes and no modifications or adaptations are made. (see below)

\section{Use by commercial "for-profit" organizations}

Use of Wiley Open Access articles for commercial, promotional, or marketing purposes requires further explicit permission from Wiley and will be subject to a fee.

Further details can be found on Wiley Online Library http://olabout.wiley.com/WileyCDA /Section/id-410895.html

\section{Other Terms and Conditions:}

v1.10 Last updated September 2015

Questions? customercare@copyright.com or +1-855-239-3415 (toll free in the US) or +1-978-646-2777. 
From: Mike Minjock mminjock@ons.org

Subject: RE: reproduction agreement with $\mathrm{MM}$ requested edit

Date: August 1, 2018 at 8:43 AM

To: Belcher, Sarah Marie SMB208@pitt.edu

Cc: Donovan, Heidi Ann Scharf donovanh@pitt.edu

Dear Sarah,

I agree to the terms stated in your letter.

Thank you,

Mike Minjock

Licensing Manager

Oncology Nursing Society

+1-412-859-6251

mminjock@ons.org

www.ons.org

From: Belcher, Sarah Marie <SMB208@pitt.edu>

Sent: Tuesday, July 31, 2018 4:56 PM

To: Mike Minjock <mminjock@ons.org>

Cc: Donovan, Heidi Ann Scharf <donovanh@pitt.edu>

Subject: reproduction agreement with $\mathrm{MM}$ requested edit

Dear Mr. Minjock,

Thank you for working with Dr. Donovan and I to find a solution that works well for both ONS and our research and professional endeavors.

In consultation with the University of Pittsburgh's Office of Scholarly Communication and Publishing, I have drafted the attached document (also copied and pasted at the end of this message) to serve as documentation of our recent communication regarding reproduction of my previous publication in Oncology Nursing Forum in my dissertation document.

If the noted arrangements meet your approval, please reply to me via your ONS email with an affirmative, such as "I agree to the terms stated in your letter."

Thank you again for your assistance.

Regards,

Sarah Belcher

Sarah M. Belcher, PhD(c), RN, OCN®

Doctoral Candidate (DSCN-17-077-01-SCN)

Robert Wood Johnson Foundation Future of Nursing Scholar

University of Pittsburgh School of Nursing

415 Victoria Building

3500 Victoria Stree.t 
Pittsburgh, PA 15261

smb208@pitt.edu

(614) 354-2560

@SarahMBelcher

Mike Minjock

Licensing Manager

Oncology Nursing Society

125 Enterprise Drive

Pittsburgh, PA 15275-1214

412-859-6251 (phone)

mminjock@ons.org

July 31,2018

Dear Mr. Minjock,

This letter is to confirm the recent email exchanges between you, my dissertation chair, Dr. Heidi Donovan, and myself. As discussed, I am completing my doctoral dissertation at the University of Pittsburgh School of Nursing, entitled, "Characterizing Psychobehavioral Risks in Survivors of Multiple Primary Cancers." This correspondence will confirm your permission to reprint excerpts from the following publication in my dissertation:

Belcher, S. M., Low, C. A., Posluszny, D. M., Schear, R., Kramer, R. E., \& Donovan, H. S. (2017). Psychological distress, health behaviors, and benefit finding in survivors of multiple primary cancers: Results From the 2010 Livestrong survey. Oncology Nursing Forum, 44(6), 703-711.

https://doi.org/10.1188/17.ONF.703-711

The entire full text article accepted for publication by Dr. Katz on 4/17/17 will be included as an appendix. This article and the work it represents is foundational to my dissertation. Full and clear credit will be given to Oncology Nursing Forum as the publisher and copyright owner of the final published article.

The requested permission extends to any future revisions and editions of my dissertation, including non-exclusive world rights in all lanquages, to the electronic publication of $\mathrm{mv}$ 
dissertation by the University of Pittsburgh and to the prospective publication of my dissertation by ProQuest. ProQuest may supply copies of my dissertation on demand. These rights will in no way restrict republication of the material in any other form by you or by others authorized by you. Your signature on this letter will also confirm that your company owns the copyright to the above described material.

If these arrangements meet your approval, please reply to me via your ONS email with an affirmative, such as "I agree to the terms stated in your letter." Thank you in advance.

Sincerely,

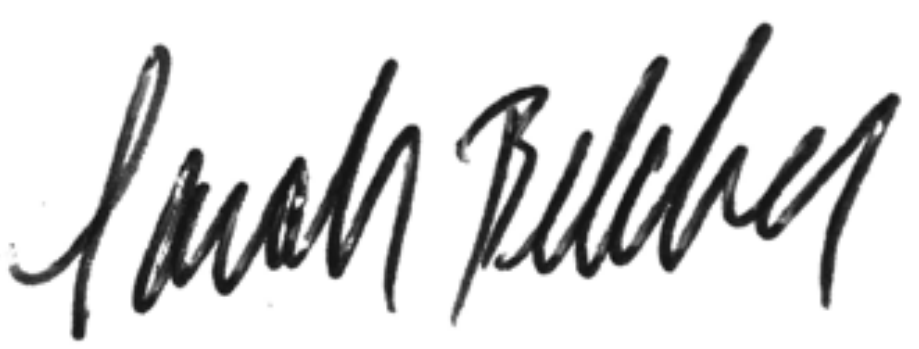

Sarah M. Belcher, $\mathrm{PhD}(\mathrm{c}), \mathrm{RN}, \mathrm{OCNO}$

Robert Wood Johnson Foundation Future of Nursing Scholars

Doctoral Candidate, School of Nursing

University of Pittsburgh

w

Belcher_ONF

article...ts.docx 


\section{University of Pittsburgh}

School of Nursing

Department of Health \& Community Systems
415 Victoria Building,

3500 Victoria Street

Pittsburgh, PA 15261

Phone: 412-624-2469

Fax: 412-383-7293

Mike Minjock

Licensing Manager

Oncology Nursing Society

125 Enterprise Drive

Pittsburgh, PA 15275-1214

412-859-6251 (phone)

mminjock@ons.org

July 31, 2018

Dear Mr. Minjock,

This letter is to confirm the recent email exchanges between you, my dissertation chair, Dr. Heidi Donovan, and myself. As discussed, I am completing my doctoral dissertation at the University of Pittsburgh School of Nursing, entitled, "Characterizing Psychobehavioral Risks in Survivors of Multiple Primary Cancers." This correspondence will confirm your permission to reprint excerpts from the following publication in my dissertation:

Belcher, S. M., Low, C. A., Posluszny, D. M., Schear, R., Kramer, R. E., \& Donovan, H. S. (2017). Psychological distress, health behaviors, and benefit finding in survivors of multiple primary cancers: Results From the 2010 Livestrong survey. Oncology Nursing Forum, 44(6), 703-711. https://doi.org/10.1188/17.ONF.703-711

The entire full text article accepted for publication by Dr. Katz on 4/17/17 will be included as an appendix. This article and the work it represents is foundational to my dissertation. Full and clear credit will be given to Oncology Nursing Forum as the publisher and copyright owner of the final published article.

The requested permission extends to any future revisions and editions of my dissertation, including nonexclusive world rights in all languages, to the electronic publication of my dissertation by the University of Pittsburgh and to the prospective publication of my dissertation by ProQuest. ProQuest may supply copies of my dissertation on demand. These rights will in no way restrict republication of the material in any other form by you or by others authorized by you. Your signature on this letter will also confirm that your company owns the copyright to the above described material.

If these arrangements meet your approval, please reply to me via your ONS email with an affirmative, such as "I agree to the terms stated in your letter." Thank you in advance.

Sincerely,<smiles>CC(C)(C)C(C)(C)C</smiles>

Sarah M. Belcher, $\mathrm{PhD}(\mathrm{c}), \mathrm{RN}, \mathrm{OCN}{ }^{\circledR}$

Robert Wood Johnson Foundation Future of Nursing Scholars

Doctoral Candidate, School of Nursing

University of Pittsburgh 


\section{APPENDIX F}

\section{DISSERTATION MANUSCRIPT 1:}

EXAMINING THE RELATIONSHIP BETWEEN MULTIPLE PRIMARY CANCERS AND PSYCHOLOGICAL DISTRESS: A REVIEW OF CURRENT LITERATURE 


\title{
Examining the relationship between multiple primary cancers and psychological distress: A review of current literature
}

\author{
Sarah M. Belcher | Emilie A. Hausmann | Susan M. Cohen | Heidi S. Donovan | \\ Elizabeth A. Schlenk
}

University of Pittsburgh School of Nursing, Pittsburgh, PA, USA

\section{Correspondence}

Sarah Belcher, University of Pittsburgh School of Nursing, 3500 Victoria Street, 415 Victoria Building, Pittsburgh, PA 15261.

Email: smb208@pitt.edu

\begin{abstract}
Objective The incidence of multiple primary cancers (MPCs) is increasing, but little is known about psychological distress in this population. The purpose of this study is to review and synthesize the literature regarding what is known about psychological distress in adults who have experienced MPC diagnoses.

Methods All potentially eligible studies identified in PubMed and CINAHL were reviewed by 2 independent evaluators, and each relevant article was assessed for methodological quality. Data were extracted, organized, and recorded using a coding log, PRISMA flow diagram, and a standardized table of evidence. Effect size (ES) values were calculated using Cohen's $d$.

Results Five of the 562 potentially relevant articles were selected for final analysis. MPC survivors, when compared with single cancer survivors, had lower global quality of life ( $d=0.32-0.37$ ), poorer emotional role function and stress $(d=0.08-0.20)$, greater and more frequent distress $(d=0.11-0.37$ ), and greater subclinical anxiety $(d=0.15)$. Depressive symptoms were variable $(d=0.01-0.22)$, and no differences between MPC and single cancer groups were identified for sleep and suicidal ideation.
\end{abstract}

Conclusion There is a substantial lack of evidence focused on psychological distress among the growing MPC survivor population. ES noted in the 5 studies reflect small but potentially significant increases in psychological distress in survivors of MPC compared with survivors of a single cancer. Clinicians should be aware of this at-risk population when screening for distress in cancer survivors. Suggestions for future research are provided.

\section{KEYWORDS}

cancer survivorship, cancer-related distress, multiple primary cancers, oncology, psychological distress, subsequent malignancies

\section{1 | BACKGROUND}

Improved cancer treatments and screening have contributed to a growing and aging cancer survivor population, and, by 2026, the number of Americans living with a history of cancer is predicted to rise to 20.3 million people. ${ }^{1-3}$ However, aging and other risk factors contribute to a $14 \%$ higher risk for cancer survivors to develop new primary cancers, when compared with the general population. Even greater risk may occur depending on the individual's site of first primary cancer, age at first cancer diagnosis, causative exposures, genetic factors, and carcinogenic effects due to cancer treatment. ${ }^{3,4}$
Cancers specifically linked to the development of subsequent malignancies, or multiple primary cancers (MPCs), include Hodgkin disease, non-Hodgkin lymphoma, and certain solid tumor (ie, prostate, testicular, ovarian, breast, and cervical) and childhood cancers. ${ }^{5,6}$ Nearly 1 in 5 cancers occurs in an individual with a previous cancer diagnosis, and MPCs are a significant cause of morbidity and mortality among cancer survivors. $^{7-9}$

All of those faced with cancer experience varying levels of distress related to diagnoses, disease- and treatment-related effects, and care transition points, ${ }^{10}$ and $20 \%$ to $47 \%$ of those with newly diagnosed and recurrent cancer experience significant distress. ${ }^{11}$ A 2011 meta- 
analysis of studies conducted in varying countries estimated that $30 \%$ to $40 \%$ of cancer patients in hospital settings have some combination of mood disorders. ${ }^{12}$ Anxiety and depression are common causes of distress in individuals with cancer with estimated prevalence of $10.3 \%$ and $14.9 \%$, respectively, both of which are higher rates than are seen in the general population. ${ }^{11,13}$ These patterns are especially concerning, considering that distress is a risk factor for nonadherence to cancer therapy, increased difficulty of treatment decision making, and is associated with poorer quality of life (QOL), poorer adherence with surveillance screening recommendations, and poorer health behaviors, such as inactivity and smoking. ${ }^{11}$

Most research examining psychological distress in cancer patients has been conducted without attention to number of primary cancers. Despite epidemiological data acknowledging the growing number of individuals with MPC, little is known regarding the relationship between MPC and psychological distress and if this distress is similar to individuals with 1 primary cancer. Lack of knowledge about the prevalence and types of psychological distress experienced by this population is an impediment to supporting their potentially unique needs. Clinically, providers need to be able to identify and target MPC survivors most at risk for psychological distress and resulting behavioral and health response sequelae.

The purpose of this study is to review and synthesize the literature regarding what is known about psychological distress in adults who have experienced MPC diagnoses. The research question being examined is: What is the relationship between experience of MPC diagnoses and psychological distress in adult cancer survivors? We hypothesized that adults who had experienced MPC diagnoses would report increased psychological distress as compared with survivors of single cancer diagnoses.

\section{2 | METHODS}

\section{1 | Selection criteria}

Criteria used to select studies for inclusion were established a priori and were as follows: (1) study participants were $\geq 18$ years old at time of the study with any type of initial primary cancer diagnosis, at least 1 additional subsequent type of primary cancer diagnosis, and in any phase of the cancer trajectory; (2) study reported on results evaluating the relationships between the presence of $\geq 2$ primary cancer diagnoses (independent variable) and psychological distress variables (dependent variable); and (3) study was published in English. No study designs were excluded.

\section{2 | Search procedures}

With expert health science librarian consultation, electronic literature searches were constructed and implemented in PubMed (which also includes Medline) ${ }^{14}$ and $\mathrm{CINAHL}^{15}$ databases, including articles from inception of databases to February 2016. Searches were built to account for variant terminology and indexing variations identified during phases of search term harvesting and testing. Searches included results for both MPC diagnoses and the responses of stress, anxiety, and/or depression. Synonyms and modified versions of terms related to MPC (eg, neoplasm, malignancy, cancer; second, multiple, metachronous; and treatment associated) and psychological distress (eg, depression, anxiety, psychological stress, and trauma) were searched to achieve the largest possible sample (search strings available online as supporting information).

\section{3 | Relevant study identification}

The resulting searches yielded 562 potentially relevant articles. Articles were reviewed for eligibility prior to analysis. In addition to the first author (S.B.), a second independent reviewer (E.H.) selected and coded articles. If article selection and/or data extraction differed between reviewers, a process of consensus-based decision making was exercised until agreement was met. ${ }^{16}$ One hundred five articles were not published in English and were therefore excluded. Initial title and abstract review was conducted on the remaining 457 articles. The 394 articles that did not meet basic selection criteria were excluded. An electronic log was created to record article details of interest and included a coding system to note rationale for each excluded article.

The remaining 63 articles received full text review. During this phase, the 2 primary reasons for exclusion were as follows: (1) MPC was discussed only as a part of post-treatment surveillance and (2) psychological distress was not evaluated separately for the MPC and single cancer diagnosis groups. No studies were identified that examined distress only within those with MPC, and no qualitative or literature synthesis studies were identified. Despite inclusion criteria that were quite broad and employment of strategic search strategies, only 3 of the 63 articles that received full review met criteria for inclusion. Hand searches of reference lists and forward citation searches of the 3 selected articles yielded 2 additional articles for analysis. See Figure 1 for a detailed description of search and article extraction processes and reasons for article exclusion.

\section{4 | Data extraction and analyses}

The 5 selected articles were reviewed and assessed for methodological quality based on the Newcastle-Ottawa Quality Assessment Scale criteria for assessing nonrandomized studies. ${ }^{17-19}$ A standardized table of evidence was created to extract, record, and appraise data from the 5 articles based on criteria relevant to this study. Effect size (ES) and 95\% confidence intervals were calculated using Cohen's $d$ to measure relationship direction and magnitude between MPC and psychological distress. ${ }^{20}$ For the articles that presented results as odds ratios, results were converted to Cohen's $d$ to allow for comparison of results between studies ${ }^{21,22}$ (Table 1). Study methodologies were compared, and results were synthesized across studies.

\section{3 | RESULTS}

Five studies met inclusion criteria for this review. Results of those studies are presented in chronological order to facilitate evaluation of the progression of scientific findings in this area. 
Unrelated topic: 255

Case study involving MPC: 73

Prevalence study involving MPC: 17

Surveillance following CA treatment: 19

Radiotherapy and MPC: 10

Biomarkers as potential indicators of MPC: 9

Treatment toxicity: 11

Excluded non-English $(n=105)$

Potentially relevant articles from primary PubMed and CINAHL databases $(\mathrm{n}=562)$

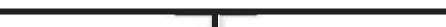

Categorization of excluded articles $(n=60)$

Surveillance following CA treatment: 19

Psychological distress not evaluated separately for 1 vs. $\geq 2$ CA: 11 Etiology of MPC development: 8

Prevalence study involving MPC: 7

Psychological distress effect of another variable: 7

Unrelated topic: 4

Case study involving MPC: 3

Biomarkers as potential indicators of MPC: 1

Articles selected for title and abstract review $(n=457)$

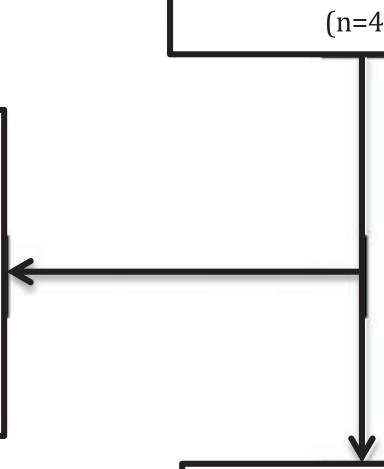

Articles selected for full-text review $(\mathrm{n}=63)$

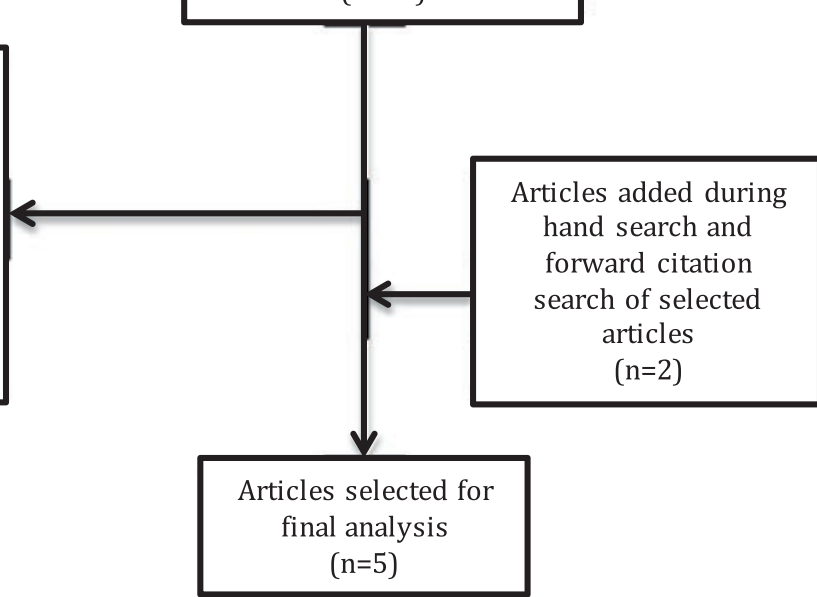

FIGURE 1 Summary of evidence search and selection

\section{1 | Gotay et al (2007)}

Gotay et $\mathrm{al}^{23}$ conducted a cross-sectional, correlational study using mailed surveys to evaluate global QOL, depressive symptoms, and cancer-specific stress after a single versus MPC diagnosis. The populationbased convenience sample of 1076 subjects was selected from the Hawaii Tumor Registry (HTR), which does not record basal and squamous cell cancers, and included 487 subjects with MPC diagnoses and 589 single cancer controls, matched on initial disease site, age, sex, race/ethnicity, time since initial diagnosis, and disease stage at initial diagnosis. Two-tailed independent sample $t$ tests were used to compare groups.

Time since first cancer diagnosis was significantly longer for MPC survivors (13.6 \pm 6.1 years) compared with those with a single cancer $(9.9 \pm 4.0$ years; $P<.05)$. Despite this difference, when compared with those with single cancers, those with MPC were still found to have significantly lower global QOL $(d=-0.37)$ as measured by a single item on the European Organization for Research and Treatment of Cancer Quality of Life Questionnaire Core 30 (EORTC QLQ-C30) and higher total cancer-specific stress $(d=0.14)$ measured by the Revised Impact 


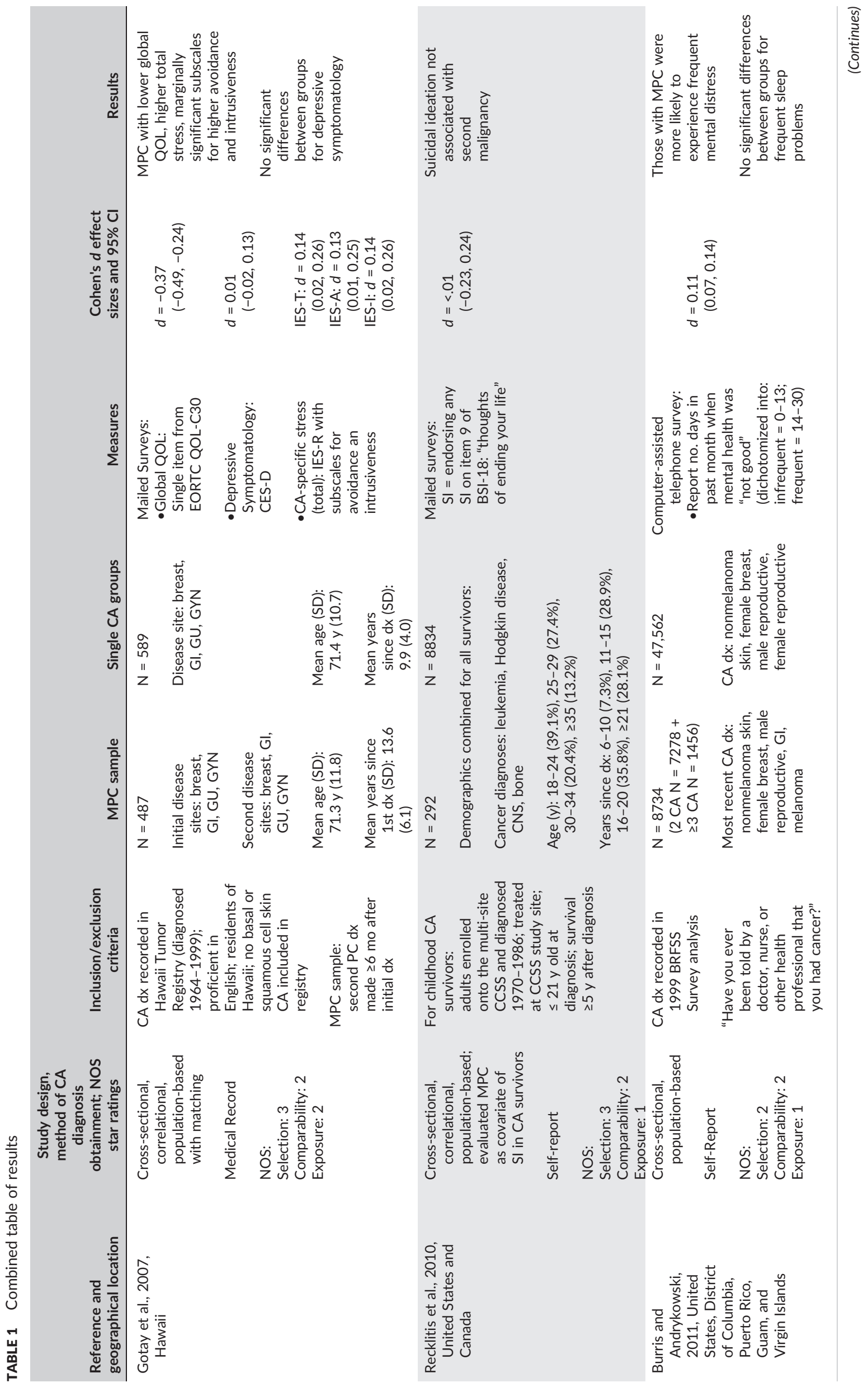




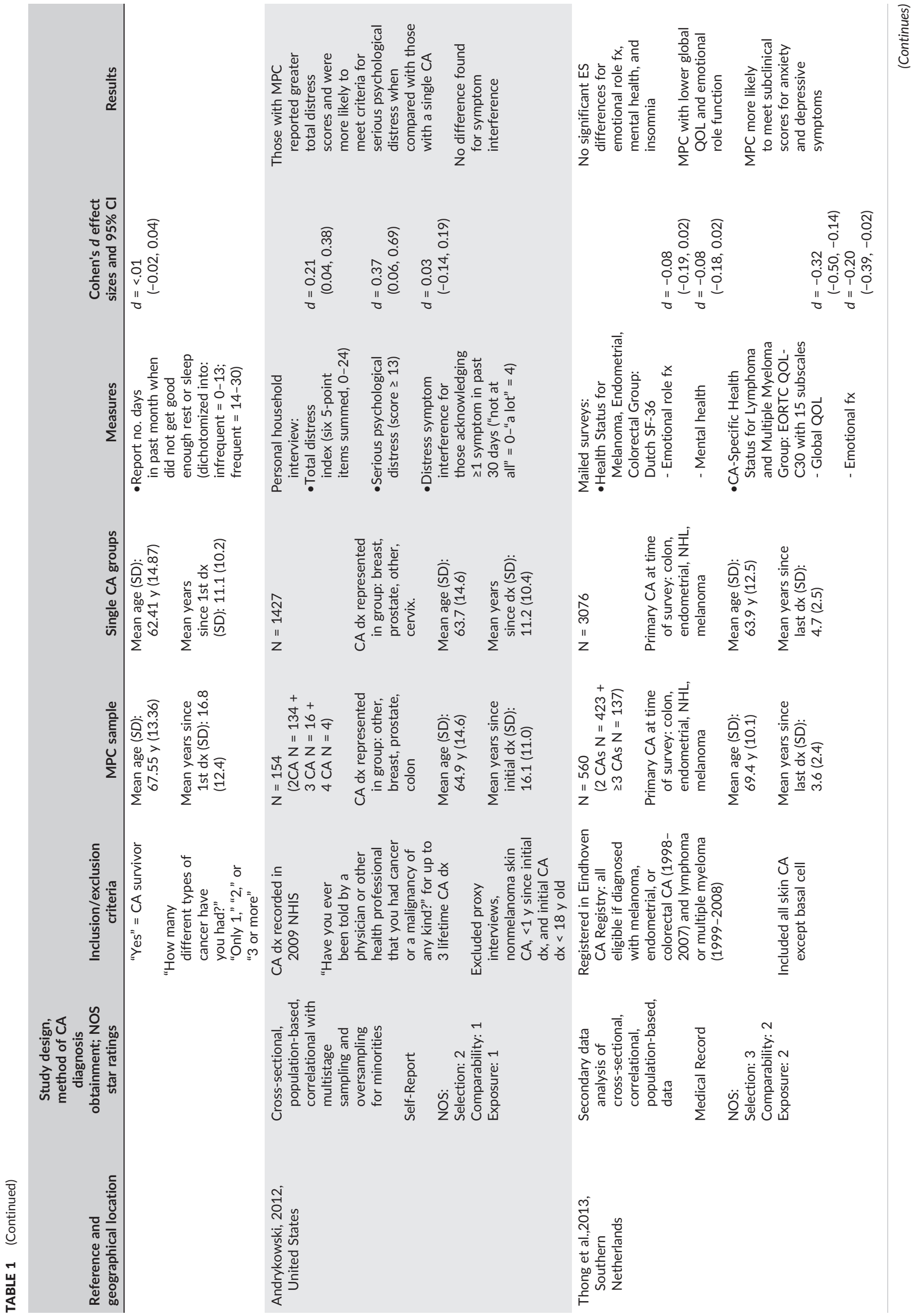




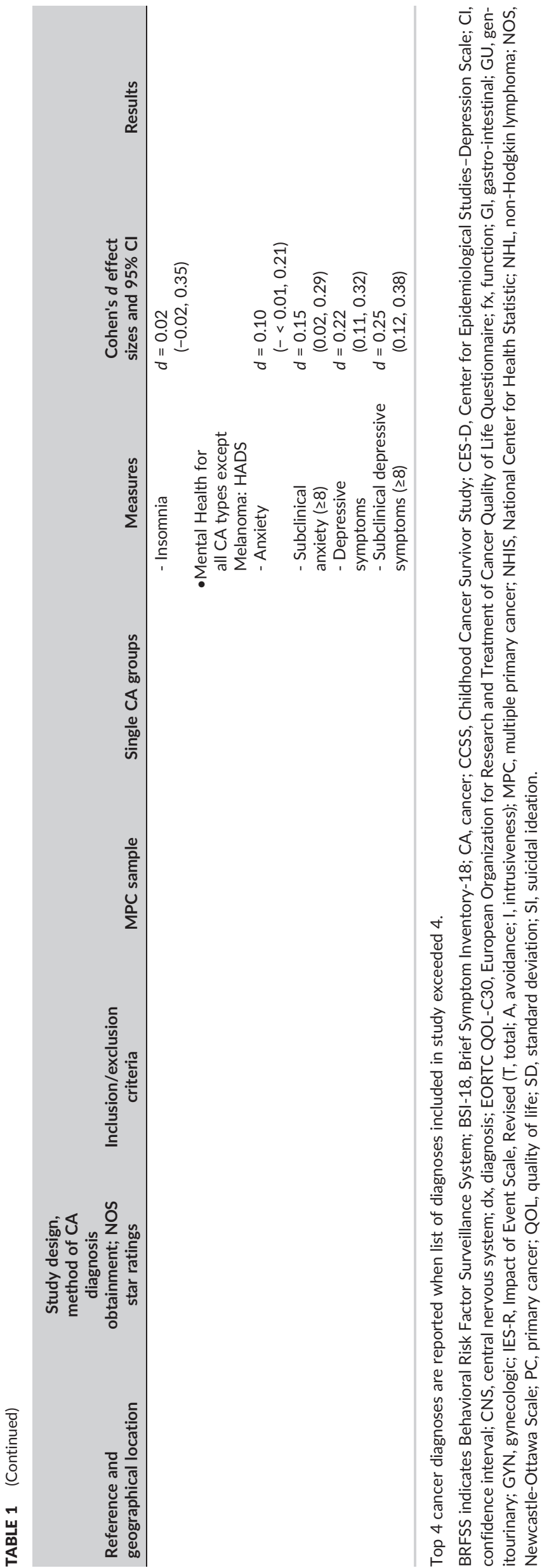

of Event Scale (IES-R). Although neither stress subscale was determined to be statistically significant $(p \leq .07)$, trends toward higher stress subscale scores for both avoidance $(d=0.13)$ and intrusiveness $(d=0.14)$ were noted. No between-group differences were identified for depressive symptomatology $(d=0.01)$ on the Center for Epidemiologic Studies-Depression (CES-D) scale.

Both the use of the HTR to identify and confirm cancer diagnoses and the use of matching for key factors between groups were strengths of this study. Limitations of this study included differences in response rates between MPC (55.8\%) and single cancer groups (41.1\%). Nonresponders differed from responders, in that responders were younger, more likely to have a partner, and more likely to report Japanese and Chinese ethnicity. In addition, individuals living with active disease were not included in this study, and prognosis was not included as a covariate in the analysis. Both of these factors could be important to understanding the impact of MPC on psychological distress. Although geographical location (Hawaii) and a large percentage of Pacific Islander participants could lead to decreased generalizability to other populations, it is a strength to focus on a relatively understudied population such as this.

\section{2 | Recklitis et al (2010)}

Recklitis et $\mathrm{al}^{24}$ published a cross-sectional, correlational study to determine the prevalence of suicidal ideation (SI) and identify correlates of SI in an adult cohort of childhood cancer survivors ( $n=9126)$ compared with their noncancer sibling controls $(n=2986)$. The population-based convenience sample of 12,112 adult subjects was selected from the Childhood Cancer Survivor Study (CCSS) from participating institutions across the United States and parts of Canada. The self-reported presence of a second malignant neoplasm in 292 cancer survivors was examined as a covariate of SI. Participants who endorsed any SI on a single item on the Brief Symptom Inventory-18 (BSI-18) about thoughts of ending one's life ("not at all" to "extremely") were considered to have SI.

Although the proportion of those endorsing SI was significantly greater in childhood cancer survivors (7.8\%), as compared with sibling controls (4.5\%; $d=0.32$ ), hierarchical logistical regression modeling, adjusted for demographic factors, cancer diagnosis, cancer treatment, and health outcomes, did not find MPC to be a significant predictor of SI in childhood cancer survivors $(d=<.01)$.

The inclusion of survivors from 2 countries is a strength of this study as is the focus on childhood cancer survivors, a group known to be at high risk for MPC development. ${ }^{3,6,25}$ Statistical regression models predicting SI were also theoretically based. Methodological concerns in this study included reliance on a single item to assess SI and self-reported health data outcomes including health and cancer history. In addition, as MPC is treated as a covariate rather than primary outcome in this study, demographic information for those with MPC is not reported separately.

\section{3 | Burris and Andrykowski (2011)}

Burris and Andrykowski ${ }^{26}$ published a cross-sectional, case-control study aimed at assessing the physical and psychological health status and health behaviors of survivors of MPC. The population-based convenience sample of 404,525 subjects was selected from the 2009 
Behavioral Risk Factor Surveillance System survey, which used a computer-assisted, random-digit dialing method and included 8734 subjects with 2 or more primary cancer diagnoses, 47,562 subjects with a single cancer diagnosis, and 348,229 controls with no cancer history. The survey was conducted throughout the continental United States, District of Columbia, Puerto Rico, Guam, and Virgin Islands. Cancer survivors whose first cancer occurred during childhood ( $<18$ years old) were excluded. Participants were asked to report the number of days in the past month when their mental health was "not good" as well as number of days in the past month they did not get enough rest or sleep (both on a 0-30 scale). Responses for unhealthy days and days of sleep problems were also dichotomized into infrequent (0-13) or frequent (14-30). Analyses of covariance (ANCOVA) and binomial logistic regression analyses were used to compare differences among groups on continuous and categorical outcomes, respectively, controlling for significant covariates.

Those with MPC differed from single cancer survivors by age at first cancer diagnosis ( $50.9 \pm 15.9$ years vs $52.0 \pm 16.0 ; P<.001)$ and time since original cancer diagnosis ( $16.9 \pm 12.4$ vs $11.1 \pm 10.2$ years; $P<.001)$. Analyses of categorical outcomes, adjusted for age, sex, race/ethnicity, marital/partner status, body mass index (BMI), and education, found that those with MPC, compared with those with single cancers, were more likely to experience frequent mental distress $(d=0.11)$ but were no more likely to experience frequent days of sleep problems $(d=<.01)$. Result patterns reported for number of days of mental distress and sleep problems were the same when analyzed continuously.

The large international sample, rigorous recruitment strategies (eg, including unlisted numbers), and controlling for significant covariates were strengths of the study. However, cancer diagnoses were obtained via self-report, and no information was provided regarding treatment history and disease staging. Individuals with a pediatric cancer history were excluded despite having similar risks for MPC, limiting generalizability to adult pediatric cancer survivors with MPC. The inclusion of nonmelanoma skin cancers may also bias data, as psychosocial problems in individuals with these types of cancers have not been shown to differ from those in the general population. ${ }^{27}$

\section{4 | Andrykowski (2012)}

Andrykowski's $2012^{28}$ cross-sectional, population-based correlational study utilized data from the 2009 National Health Information Survey to compare health status of individuals with MPC, single cancers, and no previous cancers. Multistage sampling, including oversampling for minorities, was conducted in the United States via personal household interviews. The number of cancer diagnoses was obtained via self-report. The sample included 154 respondents with 2 or more primary cancer diagnoses, 1427 respondents with a single cancer diagnosis, and 25,004 respondents with no previous cancer history. Exclusion criteria in cancer survivors included data obtained via proxy interview, being less than 1 year since initial cancer diagnosis, and being $<18$ years old at initial cancer diagnosis. Nonmelanoma skin cancer diagnoses were also excluded from this study. Participants were asked to recall feelings during the previous 30 days (sad, nervous, restless or fidgety, hopeless, everything was an effort, and worthless) and rate the 6 items on a 5-point scale, "none of the time" to "all of the time." The researchers summed the items to create a 0 to 24 total distress score. A cutoff score of $\geq 13$ was selected to indicate serious psychological distress. Participants who endorsed responses of at least "a little of the time" for at least 1 of the 6 items were asked, "How much did these feelings interfere with your life or activities," with choices on a 4-point scale from "not at all" to "a lot." Demographic variables that differed by group were included as covariates in subsequent analyses. ANCOVA and binary logistic regression were used to compare groups, controlling for difference between groups in time since initial cancer diagnosis.

MPC survivors differed from single cancer survivors in age at initial diagnosis (48.8 vs 52.5 years; $P<.01$ ) and time since initial cancer diagnosis (16.2 vs 11.2 years; $P<.001)$. When comparing MPC with single cancers, those with MPC had greater total distress index scores $(d=0.21)$ and were more likely to meet criteria for serious psychological distress $(d=0.37)$. No difference was found for symptom interference of psychological distress between groups $(d=0.03)$.

Sample size, sampling procedures to increase inclusion of minorities, and exclusion of nonmelanoma skin cancers were study strengths. Again, however, cancer diagnosis was obtained via self-report, no information was provided regarding treatment history and disease staging, and individuals with a pediatric cancer history were excluded. In addition, the distress score measure generated for this study limits ability to compare it with other studies.

\section{5 | Thong et al (2013)}

To evaluate health status and psychosocial well-being of MPC versus single cancer survivors, Thong et $\mathrm{al}^{29}$ conducted a cross-sectional secondary data analysis of 5 large cancer survivorship studies. Parent studies were conducted through the Patient Reported Outcomes Following Initial treatment and Long-term Evaluation of Survivorship (PROFILES) Eindhoven Cancer Registry in the Netherlands ${ }^{30}$ and began mailed survey data collection between 2008 and 2009. The sample for the MPC study included 3637 subjects either diagnosed with melanoma, endometrial, or colorectal cancer between 1998 and 2007 or lymphoma or multiple myeloma between 1999 and 2008. The MPC group was comprised of individuals with other primary cancers (excluding basal cell cancer) that existed in this group of cancer survivors, either before or after the cancer for which they were originally included in parent studies. The final sample included 560 subjects with MPC diagnoses and 3077 controls with a single cancer diagnosis. Chi-square tests were used to compare demographic and clinical characteristics between groups. ANCOVA, controlling for a priori determined confounding variables (age at survey, years since last diagnosis, sex, marital status, comorbidity at survey, last type of received treatment, $\mathrm{BMI}$, and $\mathrm{MPC}$ versus single cancer $\times$ years since last diagnosis interaction term), was used to compare MPC and single cancer group scores on the Dutch Short Form-36 (SF-36), EORTC QLQ-C30, and Hospital Anxiety and Depression Scale (HADS). Associations between sociodemographic, clinical, and psychological variables on SF-36 and EORTC QLQ-C30 scores were investigated using multivariate linear regression modeling. 
There were no statistically significant group differences in percentage of responders, nonresponders, and those with nonverified mailing addresses for presence of MPC at the time of the survey, but those with nonverified addresses were younger, had experienced more years since last diagnosis, and differed by type of last diagnosed primary cancer. In the participant groups, compared with those with 1 cancer, those with MPC were more likely to be older at time of survey (69.4 \pm 10.1 years vs $63.9 \pm 12.5 ; P<.0001)$, have had fewer mean years since last cancer diagnosis $(3.6 \pm 2.4$ vs $4.7 \pm 2.5 ; P<.0001)$, be retired or not working ( $87 \%$ vs $75 \% ; P<.0001$ ), and report significantly higher rates of heart disease ( $19 \%$ vs $13 \%$; $P=.0001)$, diabetes mellitus ( $14 \%$ vs $9 \% ; P=.0004)$, and stomach disease ( $2 \%$ vs $1 \%$; $P=.008)$. In the melanoma, endometrial, and colorectal group, mean scores for individuals with MPC did not differ significantly from those with single cancer on the Dutch SF-36 subscales of emotional role function ( $d=-0.08$ ) or mental health $(d=-0.08)$. As in the Gotay et $\mathrm{al}^{23}$ study, individuals with MPC in the lymphoma and multiple myeloma group had lower EORTC QLQ-C30 mean scores for global QOL $(d=-0.32)$. Those with MPC also had lower mean scores for emotional function $(d=-0.20)$ than those in the single cancer group. Similar to the Burris and Andrykowski study, ${ }^{26}$ the groups in this study did not differ in regard to insomnia $(d=0.02)$. The HADS was used to measure anxiety and depression for endometrial and colorectal cancer, Hodgkin and non-Hodgkin lymphoma, and multiple myeloma survivors. MPC and single cancer groups did not differ on mean anxiety scores $(d=0.10)$. Survivors of MPC, compared with single cancer survivors had higher depressive symptom scores $(d=0.22)$, and significantly more survivors of MPC met the established cutoff scores of $\geq 8$ both for anxiety $(d=0.15)$ and subclinical depressive symptoms $(d=0.25)$ compared with those with single cancers, again with small but potentially important ES noted.

High respondent survey response rate $(70 \%)$ and ability to rely on tumor registry information for disease and treatment variables are major strengths of this study. The use of different instruments to measure health status variables limits interpretation across disease types and potentially allows for underestimation of psychological distress in this study. This study also does not report on whether participants were undergoing active treatment at the time of the survey.

\section{6 | Synthesis across studies}

In summary, varying measures were used to capture cross-sectional data on 10,227 MPC survivors across the 5 studies. Work by Andrykowski et al ${ }^{26,28,29}$ has provided most of the scientific literature evaluating the relationship between MPC and psychological distress, and, aside from one, ${ }^{23}$ all of the studies reviewed were conducted using large, preexisting data sets. MPC survivors, when compared with single cancer survivors, had lower global QOL $(d=0.32-0.37)$, poorer emotional role function and stress $(d=0.08-0.20)$, greater and more frequent distress ( $d=0.11-0.37)$, and greater subclinical anxiety $(d=0.15)$. Depressive symptoms were variable $(d=0.01-0.22)$, and no differences between MPC and single cancer groups were identified for sleep and suicidal ideation. Using Cohen's conventions for ES, ${ }^{20,22}$ the 5 studies reviewed support a small ( $d=0.10$ to 0.37 ) increase in psychological distress in survivors of MPC, as compared with survivors of a single cancer. Questions remains as to whether this effect goes beyond theoretical interest and has clinical importance for cancer survivors, who within the MPC survivor population is most at risk for psychological distress, how the trajectory may vary over time, and long-term implications of distress in this population.

Strengths of these 5 studies include being large, population-based studies with strong designs and inclusion of a broad range of cancer types with participants residing in various locations. Consistent findings across studies despite the use of different psychological distress measures increase the generalizability of and confidence in these findings.

Limitations in this body of literature are noted. First, the inclusion of a diverse range of cancer diagnoses may make the aggregation of findings across different types of cancer problematic. Studies also differed as to whether they included subjects with histories of childhood and nonmelanoma skin cancers and participants undergoing active treatment. A primary limitation of these studies includes an overemphasis on cross-sectional survey studies, preventing ability to both establish causality of psychological distress and to determine how and under what conditions psychological distress changes. Additional major limitations are (1) a reliance on self-reporting of cancer diagnoses (reliability of self-reported cancer diagnosis has been found to vary by age, race, education, disease types/sites, and culture) $)^{31-34}$ and (2) lack of racial and ethnic diversity. Given that prevalence of psychological distress and access to support services differs across racial and ethnic groups, the homogeneity of the samples in the majority of studies examined in this review limits generalizability of the findings. ${ }^{35,36}$ Aside from the Gotay study conducted in Hawaii, ${ }^{23}$ participants of non-White race and ethnicity are not well represented in the MPC literature.

\section{4 | CONCLUSIONS}

Although this search yielded too few articles to draw strong conclusions, the identified patterns across studies support the original hypothesis that those with MPC appear to experience greater amounts of psychological distress than those with a single primary cancer. Important too was the critical lack of MPC literature identified. Despite the large sample of MPC survivors represented in this analysis, there remains a striking paucity of research describing psychological distress and the experiences of those in the growing MPC survivor population. Current Commission on Cancer guidelines on psychosocial distress screening ${ }^{37}$ are aimed at addressing cancer patients' psychosocial issues and include distress and psychosocial health needs screening as a standard of high-quality cancer care. The current knowledge gap in MPC science prevents clinicians and researchers from being able to adequately identify MPC survivors most at risk for distress and from developing targeted interventions to reduce distress and promote well-being for those most vulnerable to distress and resulting negative health sequelae. However, the use of National Comprehensive Cancer Network guidelines for distress screening, with special attention to previous cancer history, can allow for early identification of distress by clinicians and improvements in medical management. ${ }^{11,38}$ Systematic screening for previous primary cancers at time of new primary cancer diagnosis can be an additional cue to monitor for distress. 
Researchers can build upon the findings in these preliminary studies to move the science forward to develop more robust, high-quality studies. Future work in this area should include recruitment of diverse samples with particular emphasis on multiple racial and ethnic groups, verification of the number of primary cancer diagnoses via medical record review, use of uniform measurements of psychological distress that have demonstrated validity and reliability in diverse populations, ${ }^{39,40}$ exclusion of nonmelanoma skin cancers from primary cancer count, and exploration of differences within the MPC survivor population. Another important gap in the literature is whether MPC survivors who experienced initial cancer during childhood have different psychological risks compared with MPC survivors who experience cancer diagnoses only as adults. Moreover, future work should include longitudinal designs to determine if and under what conditions these findings may change over time. Despite modest ES in the identified studies, MPC survivors appear to represent a group at risk for additional psychological distress associated with MPC development. Next steps to move the science forward should include studies that discern why and under what conditions those with MPC are at increased risk and implications for distress on health outcomes. Being able to identify those within this population who are most vulnerable to negative outcomes will allow for targeted intervention work aimed at improving outcomes.

Strengths of this review include systematic research methodology consisting of a priori hypotheses and inclusion/ exclusion criteria, construction of a robust literature search in reputable databases, hand searches and forward citation searches of selected articles (resulting yield of only 2 additional relevant articles increases confidence that the 5 highly recognized, highly cited articles selected represent the current body of literature in this area), and use of 2 independent reviewers with consensus-based decision making. Systematic record keeping, data extraction, and analysis; inclusion of a PRISMA flow diagram; methodological study quality assessment; and ES calculations to allow for estimates of relationship direction and magnitude also add strength to this study. A potential limitation of this review is exclusion of articles not published in English. This analysis will hopefully spur additional research to understand and address the psychological needs of this growing population of MPC survivors. Further research should address the scientific and clinical need to understand the experiences of the expanding population of individuals with MPC.

\section{ACKNOWLEDGMENTS}

This study was supported by the Robert Wood Johnson Foundation Future of Nursing Scholars program (Belcher). The authors thank Mary Lou Klem, PhD, MLIS, for assistance with the electronic literature search and Susan Sereika, PhD, for statistical consultation.

Portions of this study have been presented in abstract and poster forms at the Cancer Survivorship Symposium, San Francisco, CA, on January 16, 2016.

\section{REFERENCES}

1. De Moor JS, Mariotto AB, Parry C, et al. Cancer survivors in the United States: prevalence across the survivorship trajectory and implications for care. Cancer Epidemiol Biomarkers Prev. 2013;22(4):561-570. doi: 10.1158/1055-9965

2. DeSantis CE, Lin CC, Mariotto AB, et al. Cancer treatment and survivorship statistics, 2014. CA Cancer J Clin. 2014;64(4):252-271. doi: 10.3322/caac. 21235

3. American Cancer Society. Cancer Treatment \& Survivorship Facts and Figures 2016-2017. 2016 Atlanta, GA: American Cancer Society.

4. Fraumeni JF Jr, Curtis RE, Edwards BK, Tucker MA. Introduction. In: Curtis RE, Freedman DM, Ron E, et al., eds. New Malignancies among Cancer Survivors: SEER Cancer Registries, 1973-2000. Bethesda, MD: National Cancer Institute; 2006:1-7.

5. American Cancer Society. Second Cancers Caused by Cancer Treatment; 2012. http://www.cancer.org/cancer/cancercauses/othercarcinogens/ medicaltreatments/secondcancerscausedbycancertreatment/index. Accessed April 20, 2015

6. Meadows AT, Friedman DL, Neglia JP, et al. Second neoplasms in survivors of childhood cancer: findings from the Childhood Cancer Survivor Study cohort. J Clin Oncol. 2009;27(14):2356-2362. doi: 10.1200/JCO.2008.21.1920

7. National Cancer Institute. Second primary cancers. http://dceg.cancer.gov/research/what-we-study/second-cancers Accessed April 4, 2016.

8. De Gonzalez AB, Curtis RE, Kry SF, et al. Proportion of second cancers attributable to radiotherapy treatment in adults: a cohort study in the US SEER cancer registries. Lancet Oncol. 2011;12(4):353-360. doi: 10.1016/S1470-2045(11)70061-4

9. Morton LM, Onel K, Curtis RE, Hungate EA, Armstrong GT. The rising incidence of second cancers: patterns of occurrence and identification of risk ractors for children and adults. In: Dizon DS, ed. 2014 American Society of Clinical Oncology Educational Book. Alexandria, VA: American Society of Clinical Oncology;2014:e57-e67. www. asco.org/edbook

10. National Comprehensive Cancer Network. NCCN Clinical Practice Guidelines in Oncology: distress Management Version 3.2015. 2015. http://www.nccn.org/professionals/physician_gls/f_guidelines.asp. Accessed January 15, 2016.

11. Holland JC, Anderson B, Breibart WS, et al. Distress management: clinical practice guidelines in oncology. I Natl Compr Canc Netw. 2013;11(2):190-209.

12. Mitchell AJ, Chan M, Bhatti $\mathrm{H}$, et al. Prevalence of depression, anxiety, and adjustment disorder in oncological, haematological, and palliativecare settings: a meta-analysis of 94 interview-based studies. Lancet Oncol. 2011;12(2):160-174. doi: 10.1016/S1470-2045(11)70002-X

13. Jacobsen PB, Andrykowski MA. Tertiary prevention in cancer care. Am Psychol. 2015;70(2):134-145. doi: 10.1037/a0036513.supp

14. U.S. National Library of Medicine. PubMed Tutorial. US National Library of Medicine. 2015. https://www.nlm.nih.gov/bsd/disted/ pubmedtutorial/cover.html Accessed July 1, 2015.

15. EBSCO Publishing. CINAHL. http://support.ebsco.com.pitt.idm.oclc. org/help/index.php?help_id=DB:62\#A Accessed September 29, 2016.

16. Liberati A, Altman DG, Tetzlaff J, et al. The PRISMA Statement for reporting systematic reviews and meta-analyses of studies that evaluate health care interventions: explanation and elaboration. Ann Intern Med. 2009;151(4):W65-W94. doi: 10.1371/journal. pmed.1000100

17. Wells GA, Shea B, O'Connell D, et al. Our research: the NewcastleOttawa Scale (NOS) for assessing the quality of non-randomized studies in meta-analyses. 2014. http://www.ohri.ca/programs/clinical_epidemiology/oxford.asp Accessed May 20, 2008.

18. Reeves BC, Deeks JJ, Higgins JPT, Wells GA. Including non-randomized studies. In: Higgins JPT, Green S, eds. Cochrane Handbook for Systematic Reviews of Interventions. 5.0.1 The Cochran Collaboration; 2008:13.1-13.34. Available from www.handbook.cochrane.org

19. Deeks JJ, Dinnes J, D'Amico R, et al. Evaluating non-randomised intervention studies. Health Technol Assess. 2003;7(27):1-192. 
20. Cohen J. The $t$ test for means. In: Statistical Power Analysis for the Behavioral Sciences. Hillsdale, NJ: Lawrence Erlbaum Associates, Publishers; 1988:19-74.2nd ed.

21. Wilson DB. Practical meta-analysis effect size calculator. The Campbell Collaboration: What Helps? What Harms? Based on what Evidence?. http://www.campbellcollaboration.org/resources/effect_size_ input.php Accessed November 17, 2015.

22. Fritz CO, Morris PE, Richler JJ. Effect size estimates: current use, calculations, and interpretation. J Exp Psychol Gen. 2012;141(1):2-18. doi: $10.1037 / \mathrm{a} 0024338$

23. Gotay CC, Ransom S, Pagano IS. Quality of life in survivors of multiple primary cancers compared with cancer survivor controls. Cancer. 2007;110(9):2101-2109. doi: 10.1002/cncr.23005

24. Recklitis CJ, Diller LR, Li X, Najita J, Robison LL, Zeltzer L. Suicide ideation in adult survivors of childhood cancer: a report from the childhood cancer survivor study. J Clin Oncol. 2010;28(4):655-661. doi: $10.1200 / J C O .2009 .22 .8635$

25. Turcotte LM, Whitton JA, Friedman DL, et al. Risk of subsequent neoplasms during the fifth and sixth decades of life in the Childhood Cancer Survivor Study cohort. J Clin Oncol. 2015; Published1-13. doi: 10.1200/JCO.2015.60.9487

26. Burris JL, Andrykowski MA. Physical and mental health status and health behaviors of survivors of multiple cancers: a national, population-based study. Ann Behav Med. 2011;42:304-312. doi: 10.1007/ s12160-011-9290-0

27. Holfeld KI, Hogan DJ, Eldemire M, Lane PR. A psychosocial assessment of patients with basal cell carcinoma. J Dermatol Surg Oncol. 1990;16(8):750-753.

28. Andrykowski MA. Physical and mental health status of survivors of multiple cancer diagnoses: findings from the National Health Interview Survey. Cancer. 2012;118(14):3645-3653. doi: 10.1002/ cncr.26678

29. Thong MSY, Mols F, Verhoeven RHA, et al. Multiple primary cancer survivors have poorer health status and well-being than single primary cancer survivors: a study from the population-based PROFILES registry. Psychooncology. 2013;22:1834-1842. doi: 10.1002/ pon.3227

30. van de Poll-Franse LV, Horevoorts N, van Eenbergen M, et al. The patient reported outcomes following initial treatment and long term evaluation of survivorship registry: scope, rationale and design of an infrastructure for the study of physical and psychosocial outcomes in cancer survivorship cohorts. Eur J Cancer. 2011;47(14):2188-2194. doi: 10.1016/j.ejca.2011.04.034

31. Abraham L, Geller BM, Yankaskas BC, et al. Accuracy of selfreported breast cancer among women undergoing mammography. Breast Cancer Res Treat. 2009;118(3):583-592. doi: 10.1007/ s10549-009-0375-4
32. Bergmann MM, Calle EE, Mervis CA, Miracle-McMahill HL, Thun MJ, Heath CW. Validity of self-reported cancers in a prospective cohort study in comparison with data from state cancer registries. Am J Epidemiol. 1998;147(6):556-562. http://www.ncbi.nlm.nih.gov/ pubmed/9521182

33. Inoue M, Sawada N, Shimazu T, et al. Validity of self-reported cancer among a Japanese population: recent results from a populationbased prospective study in Japan (JPHC Study). Cancer Epidemiol. 2011;35(3):250-253. doi: 10.1016/j.canep.2010.12.002

34. Yoshinaga A, Sasaki S, Tsugane S. Sensitivity of self-reports of cancer in a population-based prospective study: JPHC Study Cohort I. J Clin Epidemiol. 2001;54(7):741-746. http://www.ncbi.nlm.nih.gov/ pubmed/11438416

35. National Comprehensive Cancer Network. NCCN Clinical Practice Guidelines in Oncology: Distress Management Version 2.2016. 2016. https://www.nccn.org/professionals/physician_gls/pdf/distress.pdf

36. Traeger L, Cannon S, Keating NL, et al. Race by sex differences in depression symptoms and psychosocial service use among non-Hispanic black and white patients with lung cancer. J Clin Oncol. 2014;32(2):107-113. doi: 10.1200/jco.2012.46.6466

37. Commission on Cancer. Cancer program standards: ensuring patientcentered care. 2015:1-83. https://www.facs.org/qualityprograms/ cancer/coc/standards

38. Carlson LE, Bultz BD. Cancer distress screening: needs, models, and methods. J Psychosom Res. 2003;55(5):403-409. doi: 10.1016/ S0022-3999(03)00514-2

39. National Institute of Health. PROMIS Frequently Asked Questions (FAQs). http://www.nihpromis.org/faqs Accessed January 1, 2016.

40. Ashing KT, Loscalzo M, Burhansstipanov L, Huei-yu Wang J, Napoles A. Attending to distress as part of quality, comprehensive cancer care: gaps and diversity considerations. Expert Rev Life Canc Care. 2016;1(4):257-259. doi: 10.1080/23809000.2016.1195689

\section{SUPPORTING INFORMATION}

Additional Supporting Information may be found online in the supporting information tab for this article.

How to cite this article: Belcher SM, Hausmann EA, Cohen SM, Donovan HS, Schlenk EA. Examining the relationship between multiple primary cancers and psychological distress: A review of current literature. Psycho-Oncology. 2017;26: 2030-2039. https://doi.org/10.1002/pon.4299 
APPENDIX G

\section{DISSERTATION MANUSCRIPT 2:}

PSYCHOLOGICAL DISTRESS, HEALTH BEHAVIORS, AND BENEFIT FINDING IN SURVIVORS OF MULTIPLE PRIMARY CANCERS: RESULTS FROM THE 2010

LIVESTRONG SURVEY 
Manuscript 2 is unable to be published in this document due to copyright constraints.

Please refer to following link to access the full document via the publisher's website: https://onfons-org.pitt.idm.oclc.org/onf/44/6/psychological-distress-health-behaviors-and-benefit-findingsurvivors-multiple-primary. 
APPENDIX H

COVER LETTER FOR UNPUBLISHED MANUSCRIPT 3 
Shelley Blozis, PhD

Senior Statistical Editor

Health Psychology

American Psychological Association

750 First St. NE

Washington, DC 20002-4242

August 2018

Dear Dr. Blozis,

As advances are made in early detection and cancer treatment and the cancer survivor population ages, the number of individuals diagnosed with two or more, or multiple, primary cancers is also increasing. While data is known regarding risk for and prevalence of second order or higher cancer diagnoses, little is known regarding the factors linking MPC to poor health outcomes in this population and the sociodemographic and clinical factors that place individuals at risk. Previous studies have also lacked theoretical grounding. By testing a psychobehavioral stress response model, this study, entitled, "Characterizing Psychobehavioral Risks in Survivors of Multiple Primary Cancers," addresses these gaps.

This manuscript addresses a critical cancer survivorship research gap by identifying key pathways associated with health outcomes in a growing yet understudied cancer survivorship population. We also identify individual characteristics associated with these pathways to guide early identification of at risk survivors and provide guidance for advancing the science, clinical care, and policies related to patients with MPC. We believe that this manuscript is a valuable addition to the scientific literature and would fit well within the scope of Health Psychology. This manuscript addresses current research gaps, provides data on concepts and challenges for future study, and is applicable to a wide variety of researchers and clinicians alike, providing insight into the unique needs of people in the growing population of multiple primary cancer survivors.

We have followed Health Psychology's Instructions for Authors. All authors have read and approved the manuscript. This manuscript is part of the first author's dissertation research study. It is not under review elsewhere, nor does it contain data that are under review or published elsewhere, aside from the aforementioned dissertation documents. Please feel free to contact me with any questions regarding this submission.

Sincerely,

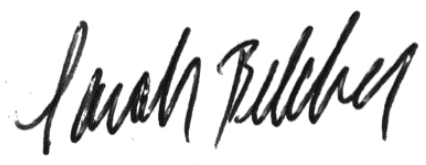

Sarah Belcher, BSN, RN, OCN ${ }^{\circledR}$

Doctoral Candidate

Robert Wood Johnson Foundation Future of Nursing Scholar 


\section{BIBLIOGRAPHY}

Abraham, L., Geller, B. M., Yankaskas, B. C., Bowles, E. J. a, Karliner, L. S., Taplin, S. H., \& Miglioretti, D. L. (2009). Accuracy of self-reported breast cancer among women undergoing mammography. Breast Cancer Research and Treatment, 118(3), 583-592. https://doi.org/10.1007/s10549-009-0375-4

Aktas, A., Walsh, D., \& Kirkova, J. (2015). The psychometric properties of cancer multisymptom assessment instruments: A clinical review. Supportive Care in Cancer, 23(7), 2189-2202. https://doi.org/10.1007/s00520-015-2732-7

American Cancer Society. (2009). Special section: Multiple primary cancers. In Cancer Facts \& Figures (pp. 24-41). Atlanta, GA: American Cancer Society.

American Cancer Society. (2012). Second Cancers Caused by Cancer Treatment. Retrieved April 20, 2015, from

http://www.cancer.org/cancer/cancercauses/othercarcinogens/medicaltreatments/secondca ncerscausedbycancertreatment/index

American Cancer Society. (2014). Cancer treatment \& survivorship: Facts \& figures 2014-2015. Atlanta, GA. Retrieved from www.cancer.org/Research/CancerFactsStatistics/cancertreatment-survivorship-facts-figures-2014-2015.pdf

American Cancer Society. (2016). Cancer treatment \& survivorship Facts and Figures 20162017. Atlanta, GA.

American Cancer Society. (2017). Cancer facts \& figures 2017. Atlanta. https://doi.org/10.1101/gad.1593107

American Psychlogical Association. (2017). Patient Health Questionnaire (PHQ-9 \& PHQ-2). Retrieved November 15, 2017, from http://www.apa.org/pi/about/publications/caregivers/practicesettings/assessment/tools/patient-health.aspx

Andersen, B. L., Kiecolt-Glaser, J. K., \& Glaser, R. (1994). A biobehavioral model of cancer stress and disease course. The American Psychologist, 49(5), 389-404. https://doi.org/10.1037/0003-066X.49.5.389

Andersen, B. L., Yang, H.-C., Farrar, W. B., Golden-Kreutz, D. M., Emery, C. F., Thornton, L. M., ... Carson III, W. E. (2008). Psychologic intervention improves survival for breast cancer patients: A randomized clinical trial. Cancer, 113(12), 3450-3458. https://doi.org/10.1002/cncr.v113

Andrykowski, M. A. (2012). Physical and mental health status of survivors of multiple cancer diagnoses: Findings from the National Health Interview Survey. Cancer, 118(14), 36453653. https://doi.org/10.1002/cncr.26678 
Aune, D., Sen, A., Prasad, M., Norat, T., Janszky, I., Tonstad, S., ... Vatten, L. J. (2016). BMI and all cause mortality: Systematic review and non-linear dose-response meta-analysis of 230 cohort studies with 3.74 million deaths among 30.3 million participants. BMJ, 353(i2156). https://doi.org/10.1136/bmj.i2156

Barlow, J., Wright, C., Sheasby, J., Turner, A., \& Hainsworth, J. (2002). Self-management approaches for people with chronic conditions: A review. Patient Education and Counseling, 48(2), 177-187. https://doi.org/10.1016/S0738-3991(02)00032-0

Barrera Jr., M., Caples, H., \& Tein, J.-Y. (2001). The psychological sense of economic hardship: Measurement models, validity, and cross-ethnic equivalence for urban families. American Journal of Community Psychology, 29(3), 493-517.

Beckjord, E. B., Reynolds, K. A., van Londen, G. J., Burns, R., Singh, R., Arvey, S. R., ... Rechis, R. (2014). Population-level trends in post-treatment cancer survivors' concerns and associated receipt of care: Results from the 2006 and 2010 LIVESTRONG surveys. Journal of Psychosocial Oncology, 32(2), 125-151. https://doi.org/10.1080/07347332.2013.874004

Begg, C. B. (1999). Methodological and statistical considerations in the study of multiple primary cancers. In A. I. Neugut, A. T. Meadows, \& E. Robinson (Eds.), Multiple Primary Cancers (pp. 13-26). Philadelphia, PA: Lippincott Williams \& Wilkins.

Belcher, S., Arida, J., Campbell, G., Hagan, T., Skrovanek, E., \& Donovan, H. S. (2015). Eastern Nursing Research Society 27th Annual Scientific Sessions abstracts: Exploring well-being, social support, and financial vulnerability in women with recurrent ovarian cancer who report more than one primary cancer diagnosis within the past 3 years. Nursing Research, 64(2), E68. https://doi.org/10.1097/NNR.0000000000000089

Belcher, S. M., Hausmann, E. A., Cohen, S. M., Donovan, H. S., \& Schlenk, E. A. (2016). Examining the relationship between multiple primary cancers and psychological distress: A review of current literature. Psycho-Oncology, 26(7), 2030-2039. https://doi.org/10.1002/pon.4299

Belcher, S. M., Low, C. A., Posluszny, D. M., Schear, R., Kramer, R. E., \& Donovan, H. S. (2017). Psychological distress, health behaviors, and benefit finding in survivors of multiple primary cancers: Results From the 2010 Livestrong survey. Oncology Nursing Forum, 44(6), 703-711. https://doi.org/10.1188/17.ONF.703-711

Belcher, S. M., Low, C., Posluszny, D., Kramer, R. E., \& Donovan, H. S. (2016). Correlates of psychological distress, health behaviors, and benefit finding in survivors of multiple primary cancers: Results from the 2010 LiveSTRONG survey. In 8th Biennial Cancer Survivorship Research: Innovation in a Rapidly Changing Landscape Abstract Book (pp. 41-42). Washington, D.C.: American Cancer Society; National Cancer Institute/National Institutes of Health; Centers for Disease Control and Prevention of the U.S. Department of Health \& Human Services; LIVESTRONG Foundation. Retrieved from www.cancer.org/subsites/Survivorship2016/2016biennialabstractbook

Belcher, S. M., Sereika, S. M., Mattos, M. K., Hagan, T. L., \& Donovan, H. S. (2017). Comparison of symptoms and quality of life in recurrent ovarian cancer by rural/urban residence: Ancillary analysis of GOG-0259 (Abstract ESGO7-0436). In 20th International 
Meeting of the European Society of Gynaecological Oncology (Vol. 27, p. 262). Vienna, Austria: International Journal of Gynecological Cancer. Retrieved from http://journals.Iww.com/ijgc/toc/2017/11004

Bergmann, M. M., Byers, T., Freedman, D. S., \& Mokdad, A. (1998). Validity of self-reported diagnoses leading to hospitalization : A comparison of self-reports with hospital records in a prospective study of American adults. American Journal of Epidemiology, 147(10), 969977. Retrieved from http://aje.oxfordjournals.org/content/147/10/969.short

Bergmann, M. M., Calle, E. E., Mervis, C. A., Miracle-McMahill, H. L., Thun, M. J., \& Heath, C. W. (1998). Validity of self-reported cancers in a prospective cohort study in comparison with data from state cancer registries. American Journal of Epidemiology, 147(6), 556-562. Retrieved from http://www.ncbi.nlm.nih.gov/pubmed/9521182

Blanchard, C. M., Courneya, K. S., \& Stein, K. (2008). Cancer survivors' adherence to lifestyle behavior recommendations and associations with health-related quality of life: Results from the American Cancer Society's SCS-II. Journal of Clinical Oncology, 26(13), 2198-2204. https://doi.org/10.1200/JCO.2007.14.6217

Bluethmann, S. M., Mariotto, A. B., \& Rowland, J. H. (2016). Anticipating the "Silver Tsunami": Prevalence trajectories and comorbidity burden among older cancer survivors in the United States. Cancer Epidemiology Biomarkers \& Prevention, 25(7), 1029-1036. https://doi.org/10.1158/1055-9965.EPI-16-0133

Bode, R. K., Hahn, E. A., Devellis, R., \& Cella, D. (2010). Measuring participation: The PatientReported Outcomes Measurement Information System experience. Archives of Physical Medicine and Rehabilitation, 91(9 Suppl), S60-S65. https://doi.org/10.1016/j.apmr.2009.10.035

Bower, J. E., \& Lamkin, D. M. (2013). Inflamation and cancer-related fatigue: Mechanisms, contributing factors, and treatment implications. Brain, Behavior, and Immunity, 30(Supplement), S48-S57. https://doi.org/10.1016/j.bbi.2012.06.011

Brookings, J. B., \& Bolton, B. (1988). Confirmatory factor analysis of the interpersonal support evaluation list. American Journal of Community Psychology, 16(1), 137-147.

Burris, J. L., \& Andrykowski, M. A. (2011). Physical and mental health status and health behaviors of survivors of multiple primary cancers: A national, population-based study. Annals of Behavioral Medicine, 42(3), 304-312. https://doi.org/10.1007/s12160-011-9290-0

Campbell, M. K., Tessaro, I., Gellin, M., Valle, C. G., Golden, S., Kaye, L., ... Jones, A. F. (2011). Adult cancer survivorship care: Experiences from the LIVESTRONG Centers of Excellence Network. Journal of Cancer Survivorship, 5(3), 271-282. https://doi.org/10.1007/s11764-011-0180-z

Cancer registry requirements. (n.d.). Retrieved May 24, 2018, from http://cancerregistrynetwork.upmc.com/upmc-network-cancer-registry/cancer-registryrequirements/

Carmack, C. L., Basen-Engquist, K., \& Gritz, E. R. (2011). Survivors at higher risk for adverse late outcomes due to psychosocial and behavioral risk factors. Cancer Epidemiology 
Biomarkers and Prevention, 20(10), 2068-2077. https://doi.org/10.1158/1055-9965.EPI-110627

Cella, D., Gershon, R., Bass, M., \& Rothrock, N. (2017). Assessment Center Scoring Service user manual. Retrieved from http://assessmentcenter.net/ac1/AssessmentCenter_Manual.pdf

Cella, D., Riley, W., Stone, A., Rothrock, N., Reeve, B., Yount, S., ... Hays, R. (2010). The patient-reported outcomes measurement information system (PROMIS) developed and tested its first wave of adult self-reported health outcome item banks: 2005-2008. Journal of Clinical Epidemiology, 63(11), 1179-1194. https://doi.org/10.1016/j.jclinepi.2010.04.011

Center for Disease Control and Prevention. (2018). Health effects of cigarette smoking. Retrieved June 11, 2018, from https://www.cdc.gov/tobacco/data_statistics/fact_sheets/health_effects/effects_cig_smokin g/index.htm

Centers for Disease Control and Prevention. (2015). The health effects of overweight and obesity. https://doi.org/10.1016/S0140-6736(14)60892-8.

Centers for Disease Control and Prevention. (2017). Overweight and obesity. https://doi.org/10.1017/S1368980017000088

Charlson, M. E., Pompei, P., Ales, K. L., \& MacKenzie, C. R. (1987). A new method of classifying prognostic comorbidity in longitudinal studies: Development and validation. Journal of Chronic Diseases, 40(5), 373-383.

Chaudhry, S., Jin, L., \& Meltzer, D. (2005). Use of a self-report-generated Charlson Comorbidity Index for predicting mortality. Medical Care, 43(6), 607-615. https://doi.org/10.1097/01.mlr.0000163658.65008.ec

Chen, Z., Koh, P. W., Ritter, P. L., Lorig, K., Bantum, E. O., \& Saria, S. (2015). Dissecting an online intervention for cancer survivors: Four exploratory analyses of internet engagement and its effects on health status and health behaviors. Health Education \& Behavior: The Official Publication of the Society for Public Health Education, 42(1), 32-45. https://doi.org/10.1177/1090198114550822

Cleeland, C. S. (2016). The M.D. Anderson Symptom Inventory User Guide, Version 1. Houston, TX: University of Texas MD Anderson Cancer Center. Retrieved from https://www.mdanderson.org/education-and-research/departments-programs-andlabs/departments-and-divisions/symptom-research/symptom-assessmenttools/MDASI_userguide.pdf

Cleeland, C. S., Mendoza, T. R., Wang, X. S., Chou, C., Harle, M. T., Morrissey, M., \& Engstrom, M. C. (2000). Assessing symptom distress in cancer patients: The M.D. Anderson Symptom Inventory. Cancer, 89(7), 1634-1646. https://doi.org/10.1002/10970142(20001001)89:7<1634::AID-CNCR29>3.0.CO;2-V

Cohen, S. (n.d.). Psychological stress, immunity and physical disease. In R. Sternberg, S. Fiske, \& D. Foss (Eds.), Scientists Making a Difference: The Greatest Living Behavioral and Brain Scientists Talk about their Most Important Contributions. New York: Cambridge 
University Press.

Cohen, S. (2008). Basic psychometrics for the ISEL 12 item scale. Retrieved March 14, 2017, from http://www.psy.cmu.edu/ scohen/Psychometrics for the ISEL 12 item scale.doc

Cohen, S., Janicki-Deverts, D., Doyle, W. J., Miller, G. E., Frank, E., Rabin, B. S., \& Turner, R. B. (2012). Chronic stress, glucocorticoid receptor resistance, inflammation, and disease risk. Proceedings of the National Academy of Sciences of the United States of America, 109(16), 5995-5999. https://doi.org/10.1073/pnas.1118355109

Cohen, S., Kamarck, T., \& Mermelstein, R. (1983). A global measure of perceived stress. Journal of Health and Social Behavior, 24(4), 385-396.

Cohen, S., Kessler, R. C., \& Underwood Gordon, L. (1995). Strategies for measuring stress in studies of psychiatric and physical disorders. In S. Cohen, R. C. Kessler, \& L. Underwood Gordon (Eds.), Measuring stress: A guide for health and social science. (pp. 3-25). New York: Oxford University Press.

Cohen, S., Mermelstein, R., Karmark, T., \& Hoberman, H. M. (1985). Measuring the functional components of social support. In I. G. Sarason \& B. R. Sarason (Eds.), Social support: Theory, research and applications (pp. 73-94). The Hague, The Netherlands: Martinus Nijhoff. https://doi.org/10.1007/978-94-009-5115-0_5

Cohen, S., Tyrrell, D. A. J., \& Smith, A. P. (1991). Psychological stress and susceptibility to the common cold. New England Journal of Medicine, 325, 606-612.

Conger, R. D., \& Elder Jr., G. H. (1994). Families in troubled times: Adapting to change in rural America. New York: Aldine de Gruyter.

Cook, K. F., Jensen, S. E., Schalet, B. D., Beaumont, J. L., Amtmann, D., Czajkowski, S., ... Cella, D. (2016). PROMIS $®$ measures of pain, fatigue, negative affect, physical function, and social function demonstrate clinical validity across a range of chronic conditions. Journal of Clinical Epidemiology, 73, 89-102. https://doi.org/10.1016/j.jclinepi.2015.08.038

Corbett, T., Cheetham, T., Müller, A. M., Slodkowska-Barabasz, J., Wilde, L., Krusche, A., ... Bradbury, K. (2018). Exploring cancer survivors' views of health behavior change: "Where do you start, where do you stop with everything?" Psycho-Oncology. https://doi.org/10.1002/pon.4732

Corbin, J. M., \& Strauss, A. (1988). The unending work and care associated with chronic illness. In Unending Work and Care: Managing Chronic Illness at Home (pp. 318-336). San Francisco, CA and London: Jossey-Bass Inc., Publishers and Jossey-Bass Limited.

Costa, D. S. J., Mercieca-Bebber, R., Rutherford, C., Gabb, L., \& King, M. T. (2016). The impact of cancer on psychological and social outcomes. Australian Psychologist, 51(2), 89-99. https://doi.org/10.1111/ap.12165

Davidson, N. E. (2017). Incident cancer in cancer survivors: When cancer lurks in the background. JAMA Oncology. https://doi.org/10.1001/jamaoncol.2017.4167

Davies, N., Batehup, L., \& Thomas, R. (2011). The role of diet and physical activity in breast, 
colorectal, and prostate cancer survivorship: A review of the literature. British Journal of Cancer, 105(10), S52-S73. https://doi.org/10.1038/bjc.2011.423

De Gonzalez, A. B., Curtis, R. E., Kry, S. F., Gilbert, E., Lamart, S., Berg, C. D., ... Ron, E. (2011). Proportion of second cancers attributable to radiotherapy treatment in adults: A cohort study in the US SEER cancer registries. The Lancet Oncology, 12(4), 353-360. https://doi.org/10.1016/S1470-2045(11)70061-4

de Leeuw, E., \& Berzelak, N. (2016). Survey mode or survey modes? In C. Wolf, D. Joye, T. W. Smith, \& Y. Fu (Eds.), The SAGE Handbook of Survey Methodology (pp. 142-156).

Thousand Oaks, CA: SAGE Publications Ltd.

De Souza, J. A., Yap, B. J., Hlubocky, F. J., Wroblewski, K., Ratain, M. J., Cella, D., \& Daugherty, C. K. (2014). The development of a financial toxicity patient-reported outcome in cancer: The COST measure. Cancer, 120(20), 3245-3253.

https://doi.org/10.1002/cncr.28814

de Souza, J. A., Yap, B. J., Wroblewski, K., Blinder, V., Araujo, F. S., Hlubocky, F. J., ... Cella, D. (2016). Measuring financial toxicity as a clinically relevant patient-reported outcome: The validation of the COmprehensive Score for financial Toxicity. Cancer, 123(3), 476484. https://doi.org/10.1002/cncr.30369

Deakin University. (2016). Health Education Impact Questionnaire (heiQ). Retrieved August 4, 2016, from http://www.deakin.edu.au/research/cphr/our-research/public-health-innovationunit/our-research

Demark-Wahnefried, W., Aziz, N. M., Rowland, J. H., \& Pinto, B. M. (2005). Riding the crest of the teachable moment: Promoting long-term health after the diagnosis of cancer. Journal of Clinical Oncology, 23(24), 5814-5830. https://doi.org/10.1200/JCO.2005.01.230

DeSantis, C. E., Lin, C. C., Mariotto, A. B., Siegel, R. L., Stein, K. D., Kramer, J. L., ... Jemal, A. (2014). Cancer treatment and survivorship statistics, 2014. CA: A Cancer Journal for Clinicians, 64(4), 252-271. https://doi.org/10.3322/caac.21235

DeWalt, D. A., Rothrock, N., Yount, S., \& Stone, A. A. (2007). Evaluation of item candidates: The PROMIS qualitative item review. Medical Care, 45, S12-S21. https://doi.org/10.1097/01.mlr.0000254567.79743.e2

Dillman, D. A., \& Edwards, M. L. (2016). Designing a mixed-mode survey. In C. Wolf, D. Joye, T. W. Smith, \& Y. Fu (Eds.), The SAGE Handbook of Survey Methodology (pp. 255-268). Thousand Oaks, CA: SAGE Publications Ltd.

Dillman, D. A., Smyth, J. D., \& Christian, L. M. (2014). Internet, phone, mail, and mixed-mode surveys: The tailored design method design (4th ed.). Hoboken, New Jersey: John Wiley \& Sons, Inc. https://doi.org/10.2307/41061275

Donnellan, M. B., Oswald, F. L., Baird, B. M., \& Lucas, R. E. (2006). The Mini-IPIP scales: Tinyyet-effective measures of the Big Five factors of personality. Psychological Assessment, 18(2), 192-203. https://doi.org/10.1037/1040-3590.18.2.192

Dowling, E. C., Chawla, N., Forsythe, L. P., de Moor, J., McNeel, T., Rozjabek, H. M., ... 
Yabroff, K. R. (2013). Lost productivity and burden of illness in cancer survivors with and without other chronic conditions. Cancer, 119(18), 3393-3401.

https://doi.org/10.1002/cncr.28214

Dowrick, C., Dixon-Woods, M., Holman, H., \& Weinman, J. (2005). What is chronic illness? Chronic IIIness, 1(1), 1-6. https://doi.org/10.1179/174239505X19572

Elsworth, G. R., Nolte, S., \& Osborne, R. H. (2015). Factor structure and measurement invariance of the Health Education Impact Questionnaire: Does the subjectivity of the response perspective threaten the contextual validity of inferences? SAGE Open Medicine, 3. https://doi.org/10.1177/2050312115585041

Enders, C. K., \& Bandalos, D. L. (2001). The relative performance of full information maximum likelihood estimation for missing data in structural equation models. Structural Equation Modeling, 8(3), 430-457.

Fleury, J. (1998). The Index of Self-Regulation: Development and psychometric analysis. Journal of Nursing Measurement, 6(1), 3-17.

Ford, E. S., Bergmann, M. M., Kroger, J., Schienkiewitz, A., Weikert, C., \& Boeing, H. (2009). Healthy living is the best revenge. Archives of Internal Medicine, 169(15), 1355-1362.

Fowler, F. J. (2014). Survey Research Methods (5th ed.). Boston, MA: SAGE Publications, Inc.

Fraumeni Jr, J. F., Curtis, R. E., Edwards, B. K., \& Tucker, M. A. (2006). Introduction. In R. E. Curtis, D. M. Freedman, E. Ron, L. A. G. Ries, D. G. Hacker, B. K. Edwards, ... J. F. Fraumeni Jr. (Eds.), New Malignancies among Cancer Survivors: SEER Cancer Registries, 1973-2000 (pp. 1-7). Bethesda, MD: National Cancer Institute.

George, D., \& Mallery, P. (2003). SPSS for Windows step by step: A simple guide and reference (4th ed.). Boston, MA: Allyn \& Bacon.

Gibbons, L. E., Fredericksen, R., Merrill, J. O., McCaul, M. E., Chander, G., Hutton, H., ... Crane, P. K. (2016). Suitability of the PROMIS Alcohol Use Short Form for screening in a HIV clinical care setting. Drug and Alcohol Dependence, 164, 113-119. https://doi.org/10.1016/j.drugalcdep.2016.04.038

Goldberg, L. R. (1999). A broad-bandwidth, public-domain, personality inventory measuring the lower-level facets of several Five-Factor models. In I. Mervielde, I. J. Deary, F. De Fruyt, \& F. Ostendord (Eds.), Personality Psychology in Europe (Vol. 7, pp. 7-28). Rilburg, The Netherlands: Tilburg University Press.

Goldberg, L. R., Johnson, J. A., Eber, H. W., Hogan, R., Ashton, M. C., Cloninger, C. R., \& Gough, H. G. (2006). The International Personality Item Pool and the future of publicdomain personality measures. Journal of Research in Personality, 40, 84-96. https://doi.org/10.1016/j.jrp.2005.08.007

Golden-Kreutz, D. M., Browne, M. W., Frierson, G. M., \& Andersen, B. L. (2004). Assessing stress in cancer patients: A second-order factor analysis model for the Perceived Stress Scale. Assessment, 11(3), 216-223. https://doi.org/10.1177/1073191104267398 
Gotay, C. C., \& Pagano, I. S. (2007). Assessment of Survivor Concerns (ASC): A newly proposed brief questionnaire. Health and Quality of Life Outcomes, 13(5:15). https://doi.org/10.1186/1477-7525-5-15

Gotay, C. C., Ransom, S., \& Pagano, I. S. (2007). Quality of life in survivors of multiple primary cancers compared with cancer survivor controls. Cancer, 110(9), 2101-2109. https://doi.org/10.1002/cncr.23005

Grady, P. A., Daley, K., \& Gough, L. (2014). The 2013 National Nursing Research Roundtable: Advancing the science of chronic illness self-management. Nursing Outlook, 62(3), 201203. https://doi.org/10.1016/j.outlook.2013.12.001

Grady, P. A., \& Gough, L. L. (2014). Self-management: A comprehensive approach to management of chronic conditions. American Journal of Public Health, 104(8), e25-e31. https://doi.org/10.2105/AJPH.2014.302041

Green McDonald, P., O'Connell, M., \& Lutgendorf, S. K. (2013). Psychoneuroimmunology and cancer: A decade of discovery, paradigm shifts, and methodological innovations. Brain, Behavior, and Immunity, 30(SUPPL.), S1-S9. https://doi.org/10.1016/j.bbi.2013.01.003

Grey, M., Knafl, K., \& McCorkle, R. (2006). A framework for the study of self- and family management of chronic conditions. Nursing Outlook, 54(5), 278-286.

https://doi.org/10.1016/j.outlook.2006.06.004

Grey, M., Schulman-Green, D., Knafl, K., \& Reynolds, N. R. (2015). A revised Self- and Family Management Framework. Nursing Outlook, 63(2), 162-170.

https://doi.org/10.1016/j.outlook.2014.10.003

Groves, R. M., Fowler, F. J., Couper, M. P., Lepkowski, J. M., Singer, E., \& Tourangeau, R. (2009). Survey Methodology (2nd ed.). Hoboken, New Jersey: John Wiley \& Sons, Inc.

Hagan, T. L., Belcher, S. M., \& Donovan, H. S. (2017). Mind the mode: Differences in paper vs. web-based survey modes among women with cancer. Journal of Pain and Symptom Management, 54(3), 368-375. https://doi.org/10.1016/j.jpainsymman.2017.07.005

Hahn, E. A., Beaumont, J. L., Pilkonis, P. A., Garcia, S. F., Magasi, S., DeWalt, D. A., \& Cella, D. (2016). The PROMIS satisfaction with social participation measures demonstrate responsiveness in diverse clinical populations. Journal of Clinical Epidemiology, 73, 135141. https://doi.org/10.1016/j.jclinepi.2015.08.034

Hahn, E. A., Cella, D., Bode, R. K., \& Hanrahan, R. T. (2010). Measuring social well-being in people with chronic illness. Social Indicators Research, 96(3), 381-401. https://doi.org/10.1007/s11205-009-9484-z

Hahn, E. A., DeWalt, D. A., Bode, R. K., Garcia, S. F., DeVellis, R. F., Correia, H., \& Cella, D. (2014). New English and Spanish social health measures will facilitate evaluating health determinants. Health Psychology, 33(5), 490-499. https://doi.org/10.1037/hea0000055

Hammer, M. J., Ercolano, E. A., Wright, F., Dickson, V. V., Chyun, D., \& Melkus, G. D. (2015). Self-management for adult patients with cancer: An integrative review. Cancer Nursing, 38(2), E10-26. https://doi.org/10.1097/NCC.0000000000000122 
Harper, F. W. K., Schmidt, J. E., Beacham, A. O., Salsman, J. M., Averill, A. J., Graves, K. D., \& Andrykowski, M. A. (2007). The role of social cognitive processing theory and optimism in positive psychosocial and physical behavior change after cancer diagnosis and treatment. Psycho-Oncology, 16(1), 79-91.

Hawkins, N. A., Smith, T., Zhao, L., Rodriguez, J., Berkowitz, Z., \& Stein, K. D. (2010). Healthrelated behavior change after cancer: Results of the American Cancer Society's studies of cancer survivors (SCS). Journal of Cancer Survivorship, 4(1), 20-32.

https://doi.org/10.1007/s11764-009-0104-3

Hays, R. D., Bjorner, J. B., Revicki, D. A., Spritzer, K. L., \& Cella, D. (2009). Development of physical and mental health summary scores from the patient-reported outcomes measurement information system (PROMIS) global items. Quality of Life Research, 18(7), 873-880. https://doi.org/10.1007/s11136-009-9496-9

HealthMeasures. (2017). Global health: A brief guide to the PROMIS Global Health instruments. Northwestern University. Retrieved from www.healthmeasures.net/images/PROMIS/manuals/PROMIS_Global_Scoring_Manual.pdf

Hershman, D. L., Greenlee, H., Awad, D., Kalinsky, K., Maurer, M., Kranwinkel, G., ... Crew, K. D. (2013). Randomized controlled trial of a clinic-based survivorship intervention following adjuvant therapy in breast cancer survivors. Breast Cancer Research and Treatment, 138(3), 795-806. https://doi.org/10.1007/s10549-013-2486-1

Holfeld, K. I., Hogan, D. J., Eldemire, M., \& Lane, P. R. (1990). A psychosocial assessment of patients with basal cell carcinoma. Journal of Dermatologic Surgery and Oncology, 16(8), 750-753.

Holland, J. C., Anderson, B., Breibart, W. S., Buchmann, L. O., Compas, B., Deshields, T. L., ... Freedman-Cass, D. A. (2013). Distress management: Clinical practice guidelines in oncology. Journal of the National Comprehensive Cancer Network: NCCN, 11(2), 190-209.

Hu, L. T., \& Bentler, P. M. (1999). Cutoff criteria for fit indexes in covariance structure analysis: Conventional criteria versus new alternatives. Structural Equation Modeling: $A$ Multidisciplinary Journal, 6(1), 1-55. https://doi.org/10.1080/10705519909540118

Inoue, M., Sawada, N., Shimazu, T., Yamaji, T., Iwasaki, M., Sasazuki, S., \& Tsugane, S. (2011). Validity of self-reported cancer among a Japanese population: Recent results from a population-based prospective study in Japan (JPHC Study). Cancer Epidemiology, 35(3), 250-253. https://doi.org/10.1016/j.canep.2010.12.002

Jacobsen, P. B., \& Andrykowski, M. A. (2015). Tertiary prevention in cancer care. American Psychologist, 70(2), 134-145. Retrieved from http://dx.doi.org/10.1037/a0036513.supp

Jemal, A., Ward, E. M., Johnson, C. J., Cronin, K. A., Ma, J., Ryerson, A. B., ... Weir, H. K. (2017). Annual Report to the Nation on the Status of Cancer, 1975-2014, featuring survival. Journal of the National Cancer Institute, 109(9), 1-22. https://doi.org/10.1093/jnci/djx030

Jensen, R. E., Potosky, A. L., Moinpour, C. M., Lobo, T., Cella, D., Hahn, E. A., ... Reeve, B. B. (2017). United States population-based estimates of Patient-Reported Outcomes 
Measurement Information System symptom and functional status reference values for individuals with cancer. Journal of Clinical Oncology, 35.

https://doi.org/10.1200/JCO.2016.71.4410

Johnson, C. H., Peace, S., Adamo, P., Fritz, A., Percy-Laurry, A., \& Edwards, B. K. (2007). The 2007 multiple primary and histology coding rules. Bethesda, MD: National Cancer Institute, Surveillance, Epidemiology and End Results Program. Retrieved from https://seer.cancer.gov/tools/mphrules/download.html

Kanera, I. M., Bolman, C. A. W., Mesters, I., Willems, R. A., Beaulen, A. A. J. M., \& Lechner, L. (2016). Prevalence and correlates of healthy lifestyle behaviors among early cancer survivors. BioMed Central Cancer, 16(4). https://doi.org/10.1186/s12885-015-2019-x

Kenny, D. A. (2015). Measuring model fit. Retrieved June 5, 2018, from http://davidakenny.net/cm/fit.htm

Kent, E. E., Forsythe, L. P., Yabroff, K. R., Weaver, K. E., de Moor, J. S., Rodriguez, J. L., \& Rowland, J. H. (2013). Are survivors who report cancer-related financial roblems more likely to forgo or delay medical care? Cancer, 119(20), 3710-3717. Retrieved from https://www.ncbi.nlm.nih.gov/pmc/articles/PMC4552354/pdf/nihms718119.pdf

Kiecolt-Glaser, J. K., Marucha, P. T., Malarkey, W. B., Mercado, a M., \& Glaser, R. (1995). Slowing of wound healing by psychological stress. The Lancet, 346(8984), 1194-1196. https://doi.org/10.1016/S0140-6736(95)92899-5

Klein, W. M. P., Bloch, M., Hesse, B. W., McDonald, P. G., Nebeling, L., O'Connell, M. E., ... Tesauro, G. (2014). Behavioral research in cancer prevention and control. American Journal of Preventive Medicine, 46(3), 303-311. https://doi.org/10.1016/j.amepre.2013.10.004

Knobf, M. T., Cooley, M. E., Duffy, S., Doorenbos, A., Eaton, L., Given, B., ... Mallory, G. (2015). The 2014-2018 Oncology Nursing Society research agenda. Oncology Nursing Forum, 42(5), 450-465. https://doi.org/10.1188/15.ONF.450-465

Koubkova, L., Hrstka, R., Dobes, P., Vojtesek, B., \& Vyzula, R. (2014). Second primary cancers: Causes, incidence and the future. Klinicka Onkologie, 27(1), 11-17.

Kroenke, K., Spitzer, R. L., \& Williams, J. B. W. (2001). The PHQ-9: Validity of a brief depression severity measure. Journal of General Internal Medicine, 16(9), 606-613. https://doi.org/10.1046/j.1525-1497.2001.016009606.x

Kroenke, K., Spitzer, R. L., \& Williams, J. B. W. (2003). The Patient Health Questionnaire-2: Validity of a two-item depression screener. Medical Care, 41(11), 1284-1292. https://doi.org/10.1097/01.MLR.0000093487.78664.3C

Kroenke, K., Spitzer, R. L., Williams, J. B. W., Monahan, P. O., \& Lowe, B. (2007). Anxiety disorders in primary care: Prevalence, impairment, comorbidity, and detection. Annals of Internal Medicine, 146(5), 317-326.

Kroenke, K., Strine, T. W., Spitzer, R. L., Williams, J. B. W., Berry, J. T., \& Mokdad, A. H. (2009). The PHQ-8 as a measure of current depression in the general population. Journal 
of Affective Disorders, 114(1-3), 163-173. https://doi.org/10.1016/j.jad.2008.06.026

Land, S. R., Toll, B. A., Moinpour, C. M., Mitchell, S. A., Ostroff, J. S., Hatsukami, D. K., ... Warren, G. W. (2016). Research priorities, measures, and recommendations for assessment of tobacco use in clinical cancer research. Clinical Cancer Research, 22(8), 1907-1913. https://doi.org/10.1158/1078-0432.CCR-16-0104

Land, S. R., Warren, G. W., Crafts, J. L., Hatsukami, D. K., Ostroff, J. S., Willis, G. B., ... Toll, B. A. (2016). Cognitive testing of tobacco use items for administration to patients with cancer and cancer survivors in clinical research. Cancer, 122(11), 1728-1734. https://doi.org/10.1002/cncr.29964

Lazarus, R. S., \& Folkman, S. (1984). The stress concept in the life sciences. In Stress, Appraisal, and Coping (pp. 1-21). New York, NY: Springer Publishing Company, Inc.

Li, C.-H. (2016). Confirmatory factor analysis with ordinal data: Comparing robust maximum likelihood and diagonally weighted least squares. Behavior Research Methods, 48(3), 936949. https://doi.org/10.3758/s13428-015-0619-7

Ligibel, J. A., Alfano, C. M., Courneya, K. S., Demark-Wahnefried, W., Burger, R. A., Chlebowski, R. T., ... Hudis, C. A. (2014). American society of clinical oncology position statement on obesity and cancer. Journal of Clinical Oncology, 32(31), 3568-3574. https://doi.org/10.1200/JCO.2014.58.4680

Little, T. D. (2013). Longitudinal Structural Equation Modeling. New York: The Guilford Press.

Lorig, K. R., \& Holman, H. R. (2003). Self-management education: History, definition, outcomes, and mechanisms. Annals of Behavioral Medicine, 26(1), 1-7.

https://doi.org/10.1207/S15324796ABM2601

Lorig, K. R., Sobel, D. S., Ritter, P. L., Laurent, D., \& Hobbs, M. (2001). Effect of a selfmanagement program on patients with chronic disease. Effective Clinical Practice: ECP, 4(6), 256-262. Retrieved from www.ecp.acponline.org

Lorig, K., Stewart, A., Ritter, P., Gonzalez, V., Laurent, D., \& Lynch, J. (1996). Construction of measures of behaviors, self-efficacy, and outcomes. In Outcome Measures for Health Education and other Health Care Interventions (pp. 10-33). Thousand Oaks, CA: SAGE Publications, Inc.

Low, C. A., Beckjord, E., Bovbjerg, D. H., Dew, M. A., Posluszny, D. M., Schmidt, J. E., ... Rechis, R. (2014). Correlates of positive health behaviors in cancer survivors: Results from the 2010 LIVESTRONG survey. Journal of Psychosocial Oncology, 32(6), 678-695. https://doi.org/10.1080/07347332.2014.955243

Löwe, B., Decker, O., Müller, S., Brähler, E., Schellberg, D., Herzog, W., \& Herzberg, P. Y. (2008). Validation and standardization of the Generalized Anxiety Disorder Screener (GAD7 ) in the general population. Medical Care, 46(3), 266-274. https://doi.org/10.1097/MLR.0b013e318160d093

Löwe, B., Kroenke, K., \& Gräfe, K. (2005). Detecting and monitoring depression with a two-item questionnaire (PHQ-2). Journal of Psychosomatic Research, 58(2), 163-171. 
https://doi.org/10.1016/j.jpsychores.2004.09.006

Mariotto, A. B., Rowland, J. H., Ries, L. A. G., Scoppa, S., \& Feuer, E. J. (2007). Multiple cancer prevalence: A growing challenge in long-term survivorship. Cancer Epidemiology Biomarkers and Prevention, 16(3), 566-571. https://doi.org/10.1158/1055-9965.EPI-060782

Mason, G. (1987). Coping with multicollinearity. The Canadian Journal of Program Evaluation, 2(1), 87-93. Retrieved from http://evaluationcanada.ca/secure/02-1-087.pdf

Matthews, K. A., \& Gump, B. B. (2002). Chronic work stress and marital dissolution increase risk of posttrial mortality in men from the Multiple Risk Factor Intervention Trial. Archives of Internal Medicine, 162(3), 309-315. https://doi.org/10.1001/archinte.162.3.309

Maunsell, E., Lauzier, S., Brunet, J., Pelletier, S., Osborne, R. H., \& Campbell, H. S. (2014). Health-related empowerment in cancer: Validity of scales from the Health Education Impact Questionnaire. Cancer, 120(20), 3228-3236. https://doi.org/10.1002/cncr.28847

McCorkle R, Ercolano E, Lazenby M, Schulman- Green D, Schilling L S, Lorig K, \& Wagner E H. (2011). Self-management: Enabling and empowering patients living with cancer as a chronic illness. CA: A Cancer Journal for Clinicians, 61(1), 50-62. https://doi.org/10.3322/caac.20093

Miller, G. E., Cohen, S., \& Ritchey, A. K. (2002). Chronic psychological stress and the regulation of pro-inflammatory cytokines: A glucocorticoid-resistance model. Health Psychology, 21(6), 531-541. https://doi.org/10.1037//0278-6133.21.6.531

Miller, K. D., Siegel, R. L., Lin, C. C., Mariotto, A. B., Kramer, J. L., Rowland, J. H., ... Jemal, A. (2016). Cancer treatment and survivorship statistics, 2016. CA: A Cancer Journal for Clinicians, 66(4), 271-289. https://doi.org/10.3322/caac.21349

Miller, S. M., Bowen, D. J., Croyle, R. T., \& Rowland, J. H. (2009). Overview, current status, and future directions. In S. M. Miller, D. J. Bowen, R. T. Croyle, \& J. H. Rowland (Eds.), Handbook of Cancer Control and Behavioral Science: A Resource for Researchers, Practitioners, and Policy Makers (First, pp. 5-22). Washington, D.C.: American Psychological Association.

Mitchell, A. J., Chan, M., Bhatti, H., Halton, M., Grassi, L., Johansen, C., \& Meader, N. (2011). Prevalence of depression, anxiety, and adjustment disorder in oncological, haematological, and palliative-care settings: A meta-analysis of 94 interview-based studies. The Lancet Oncology, 12(2), 160-174. https://doi.org/10.1016/S1470-2045(11)70002-X

Moore, S. M., Schiffman, R., Waldrop-Valverde, D., Redeker, N. S., McCloskey, D. J., Kim, M. T., ... Grady, P. (2016). Recommendations of common data elements to advance the science of self-management of chronic conditions. Journal of Nursing Scholarship, 48(5), 437-447. https://doi.org/10.1111/jnu.12233

Morton, L. M., Onel, K., Curtis, R. E., Hungate, E. A., \& Armstrong, G. T. (2014). The rising incidence of second cancers: Patterns of occurrence and identification of risk ractors for children and adults. In D. S. Dizon (Ed.), 2014 American Society of Clinical Oncology Educational Book (pp. e57-e67). Alexandria, VA: American Society of Clinical Oncology. 
Retrieved from http://meetinglibrary.asco.org/content/11400057-144

Mowls, D. S., Brame, L. S., Martinez, S. A., \& Beebe, L. A. (2016). Lifestyle behaviors among US cancer survivors. Journal of Cancer Survivorship, 10(4), 692-698.

https://doi.org/10.1007/s11764-016-0515-x

Mullan, F. (1985). Seasons of survival: Reflections of a physician with cancer. New England Journal of Medicine, 313(4), 270-273.

Mullan, F. (2016). The seasons of survival - revisited. In American Society of Clinical Oncology Cancer Survivorship Symposium. San Francisco, CA: American Society of Clinical Oncology. Retrieved from http://meetinglibrary.asco.org/content/117403?media=vm

Murphy, C. C., Gerber, D. E., \& Pruitt, S. L. (2017). Prevalence of prior cancer among persons newly diagnosed with cancer: An initial report from the Surveillance, Epidemiology, and End Results Program. JAMA Oncology, E1-E4.

https://doi.org/10.1001/jamaoncol.2017.3605

Muthén, L. K., \& Muthén, B. O. (2017). Mplus user's guide (8th ed.). Los Angeles: Muthén \& Muthén. Retrieved from

https://www.statmodel.com/download/usersguide/MplusUserGuideVer_8.pdf

National Academy of Sciences. (2006). From Cancer Patient to Cancer Survivor: Lost in Transition. (M. Hewitt, S. Greenfield, \& E. Stovall, Eds.). Washington, D.C.: The National Academies Press. Retrieved from http://www.nap.edu/catalog/11468.html

National Cancer Institute. (n.d.-a). Second primary cancers. Retrieved April 4, 2016, from http://dceg.cancer.gov/research/what-we-study/second-cancers

National Cancer Institute. (n.d.-b). SEER stat fact sheets: Cancer of any site. Retrieved July 25, 2016, from http://seer.cancer.gov/statfacts/html/all.html

National Cancer Institute. (2013). Metastatic cancer fact sheet. Retrieved May 31, 2016, from http://www.cancer.gov/about-cancer/understanding/what-is-cancer/metastatic-fact-sheet

National Cancer Institute. (2015). Environmental carcinogens and cancer risk. Retrieved June 11, 2018, from https://www.cancer.gov/about-cancer/causesprevention/risk/substances/carcinogens

National Cancer Institute. (2016). Cancer Patient Tobacco Use Questionnaire (C-TUQ). Retrieved August 4, 2016, from https://www.gemmeasures.org/Public/MeasureDetail.aspx?mid=2322\&cat=2

National Cancer Institute. (2018a). Cancer genetics risk assessment and counseling (PDQ®): Health professional version. Retrieved July 29, 2018, from https://www.cancer.gov/aboutcancer/causes-prevention/genetics/risk-assessment-pdq\#section/all

National Cancer Institute. (2018b). Cancer survivors and obesity. Retrieved June 5, 2018, from https://progressreport.cancer.gov/after/obesity

National Cancer Institute. (2018c). Financial toxicity and cancer treatment (PDQ®) - Health 
professional version. Retrieved June 5, 2018, from https://www.cancer.gov/aboutcancer/managing-care/track-care-costs/financial-toxicity-hp-pdq

National Cancer Survivorship Resource Center. (n.d.). Systems Policy and Practice : Clinical Survivorship Care Expert Panel Summit Workgroup Overview (No. Cooperative Agreement \#1U55DP003054 6). Retrieved from www.cancer.org/acs/groups/content/@editorial/documents/document/acspc-031408.pdf

National Comprehensive Cancer Network. (2015). NCCN Clinical Practice Guidelines in Oncology: Distress Management Version 3.2015. National Comprehensive Cancer Network. Retrieved from http://www.nccn.org/professionals/physician_gls/f_guidelines.asp

National Comprehensive Cancer Network. (2016). NCCN Clinical Practice Guidelines in Oncology: Distress Management Version 2.2016. National Comprehensive Cancer Network. Retrieved from https://www.nccn.org/professionals/physician_gls/pdf/distress.pdf

National Comprehensive Cancer Network. (2017). NCCN Clinical Practice Guidelines in Oncology: Survivorship Version 1.2017. National Comprehensive Cancer Network.

National Comprehensive Cancer Network. (2018a). NCCN Clinical Practice Guidelines in Oncology: Distress Management. National Comprehensive Cancer Network. Retrieved from https://www.nccn.org/professionals/physician_gls/pdf/distress.pdf

National Comprehensive Cancer Network. (2018b). NCCN Clinical Practice Guidelines in Oncology: Survivorship. National Comprehensive Cancer Network. Retrieved from https://www.nccn.org/professionals/physician_gls/pdf/survivorship.pdf

National Institute of Health. (2017). Science of behavior change. Retrieved May 18, 2017, from https://commonfund.nih.gov/behaviorchange

National Institute of Nursing Research. (n.d.). Self-management. Retrieved June 10, 2018, from https://www.ninr.nih.gov/newsandinformation/iq/self-management-workshop

National Institute on Alcohol Abuse and Alcoholism. (2017). Alcohol facts and statistics. Retrieved June 4, 2018, from https://pubs.niaaa.nih.gov/publications/AlcoholFacts\&Stats/AlcoholFacts\&Stats.pdf

National Science Foundation. (n.d.). Declining respoinse rate, rising costs. Retrieved June 10, 2018, from https://www.nsf.gov/news/special_reports/survey/index.jsp?id=question

Ness, K. K., Wall, M. M., Oakes, J. M., Robison, L. L., \& Gurney, J. G. (2006). Physical performance limitations and participation restrictions among cancer survivors: A population-based study. Annals of Epidemiology, 16(3), 197-205. https://doi.org/10.1016/j.annepidem.2005.01.009

Northwestern University. (2016). Intro to PROMIS. https://doi.org/10.1017/CBO9781107415324.004

O’Connor, M. F., Bower, J. E., Cho, H. J., Creswell, J. D., Dimitrov, S., Hamby, M. E., ... Irwin, M. R. (2009). To assess, to control, to exclude: Effects of biobehavioral factors on circulating inflammatory markers. Brain, Behavior, and Immunity, 23(7), 887-897. 
https://doi.org/10.1016/j.bbi.2009.04.005

Ory, M. G., Ahn, S., Jiang, L., Lorig, K., Ritter, P., Laurent, D. D., ... Smith, M. L. (2013). National study of chronic disease self-management: Six-month outcome findings. Journal of Aging and Health, 25(7), 1258-1274. https://doi.org/10.1177/0898264313502531

Osborne, R. H., Batterham, R., \& Livingston, J. (2011). The evaluation of chronic disease selfmanagement support across settings: The international experience of the Health Education Impact Questionnaire quality monitoring system. The Nursing Clinics of North America, 46(3), 255-270. https://doi.org/10.1016/j.cnur.2011.05.010

Osborne, R. H., Elsworth, G. R., \& Whitfield, K. (2007). The Health Education Impact Questionnaire (heiQ): An outcomes and evaluation measure for patient education and selfmanagement interventions for people with chronic conditions. Patient Education and Counseling, 66(2), 192-201. https://doi.org/10.1016/j.pec.2006.12.002

Pace, T. W. W., Hu, F., \& Miller, A. (2007). Cytokine-effects on glucocorticoid receptor function: Relevance to glucocorticoid resistance and the pathophysiology and treatment of major depression. Brain, Behavior, and Immunity, 21(1), 9-19.

Page, G. G., Corwin, E. J., Dorsey, S. G., Redeker, N. S., Mccloskey, D. J., Austin, J. K., ... Grady, P. (2018). Biomarkers as Common Data Elements for Symptom and SelfManagement Science. Journal of Nursing Scholarship, 50(3), 276-286. https://doi.org/10.1111/jnu.12378

Park, C. L., Edmondson, D., Fenster, J. R., \& Blank, T. O. (2008). Positive and negative health behavior changes in cancer survivors: A stress and coping perspective. Journal of Health Psychology, 13(8), 1198-1206. https://doi.org/10.1177/1359105308095978

Park, J., \& Look, K. A. (2018). Relationship between objective financial burden and the healthrelated quality of life and mental health of patients with cancer. Journal of Oncology Practice, 14(2), e113-e121. https://doi.org/10.1200/JOP.2017.027136

Patient-Reported Outcomes Measurement Information System. (2014). A brief guide to the PROMIS Alcohol Use instruments. Retrieved August 4, 2016, from http://www.assessmentcenter.net/documents/PROMIS Alcohol Use Scoring Manual.pdf

Patient-Reported Outcomes Measurement Information System. (2015). Physical function: A brief guide to the PROMIS Physical Function instruments. Retrieved from https://www.assessmentcenter.net/documents/PROMIS Physical Function Scoring Manual.pdf

Pearlin, L. I., Menaghan, E. G., Lieberman, M. A., \& Mullan, J. T. (1981). The stress process. Journal of Health and Social Behavior1, 22(4), 337-356.

Pilkonis, P. A., Yu, L., Dodds, N. E., Johnston, K. L., Lawrence, S. M., \& Daley, D. C. (2016). Validation of the Alcohol Use Item Banks from the Patient-Reported Outcomes Measurement Information System (PROMIS $\left.{ }^{2}\right)$. Drug and Alcohol Dependence, 161, 316322. https://doi.org/10.1016/j.drugalcdep.2016.02.014

Plummer, F., Manea, L., Trepel, D., \& McMillan, D. (2016). Screening for anxiety disorders with 
the GAD-7 and GAD-2: A systematic review and diagnostic metaanalysis. General Hospital Psychiatry, 39, 24-31. https://doi.org/10.1016/j.genhosppsych.2015.11.005

Posluszny, D. M., Dew, M. A., Beckjord, E., Bovbjerg, D. H., Schmidt, J. E., Low, C. A., ... Rechis, R. (2015). Existential challenges experienced by lymphoma survivors: Results from the 2010 LIVESTRONG Survey. Journal of Health Psychology.

https://doi.org/10.1177/1359105315576352

Pyykkönen, A. J., Räikkönen, K., Tuomi, T., Eriksson, J. G., Groop, L., \& Isomaa, B. (2010). Stressful life events and the metabolic syndrome: The prevalence, prediction and prevention of diabetes (PPP) -Botnia Study. Diabetes Care, 33(2), 378-384. https://doi.org/10.2337/dc09-1027.

Ramsey, S. D., Bansal, A., Fedorenko, C. R., Blough, D. K., Overstreet, K. A., Shankaran, V., \& Newcomb, P. (2016). Financial insolvency as a risk factor for early mortality among patients with cancer. Journal of Clinical Oncology, 34(9), 980-986. https://doi.org/10.1200/JCO.2015.64.6620

Rechis, R., Reynolds, K. A., Beckjord, E. B., Nutt, S., Burns, R. M., \& Schaefer, J. S. (2011). "I learned to live with it" is not good enough: Challenges reported by post-treatment cancer survivors in the live strong surveys a LiveStrong report, 2010. Austin, TX. Retrieved from www.livestrong.org

Redeker, N. S., Anderson, R., Bakken, S., Corwin, E., Docherty, S., Dorsey, S. G., ... Grady, P. (2015). Advancing symptom science through use of common data elements. Journal of Nursing Scholarship, 47(5), 379-388. https://doi.org/10.1111/jnu.12155

Risendal, B. C., Dwyer, A., Seidel, R. W., Lorig, K., Coombs, L., \& Ory, M. G. (2015). Meeting the challenge of cancer survivorship in public health: Results from the evaluation of the chronic disease self-management program for cancer survivors. Frontiers in Public Health, 2(214). https://doi.org/10.3389/fpubh.2014.00214

Ritter, P. L., Stewart, A. L., Kaymaz, H., Sobel, D. S., Block, D. A., \& Lorig, K. R. (2001). Selfreports of health care utilization compared to provider records. Journal of Clinical Epidemiology, 54(2), 136-141. https://doi.org/10.1016/S0895-4356(00)00261-4

Rose, M., Bjorner, J. B., Gandek, B., Bruce, B., Fries, J. F., \& Ware, J. E. (2014). The PROMIS Physical Function item bank was calibrated to a standardized metric and shown to improve measurement efficiency. Journal of Clinical Epidemiology, 67(5), 516-526. https://doi.org/10.1016/j.jclinepi.2013.10.024

Rudy, E., \& Grady, P. (2005). Biological researchers: Building nursing science. Nursing Outlook, 53(2), 88-94. https://doi.org/10.1016/j.outlook.2004.09.006

Ryan, C. L., \& Bauman, K. (2016). Educational attainment in the United States: 2015. United States Census Bureau. https://doi.org/P20-578

Ryan, P. (2009). Integrated theory of health behavior change: Background and intervention development. Clinical Nurse Specialist CNS, 23(3), 161-170; quiz 171-172. https://doi.org/10.1097/NUR.0b013e3181a42373 
S. Yousuf Zafar, \& Abernethy, A. P. (2013). Financial toxicity, part I: A new name for a growing problem. Oncology (Williston Park), 27(2), 80-149. Retrieved from

https://www.ncbi.nlm.nih.gov/pmc/articles/PMC4523887/

Salsman, J. M., Schalet, B. D., Andrykowski, M. A., \& Cella, D. (2015). The Impact of Events Scale: A comparison of frequency versus severity approaches to measuring cancerspecific distress. Psycho-Oncology, 24(12), 1738-1745.

Sandberg, S., Paton, J. Y., Ahola, S., McCann, D. C., McGuinness, D., Hillary, C. R., \& Oja, H. (2000). The role of acute and chronic stress in asthma attacks in children. Lancet, 356, 982-987. https://doi.org/10.1016/S0140-6736(00)02715-X

Schalet, B. D., Hays, R. D., Jensen, S. E., Beaumont, J. L., Fries, J. F., \& Cella, D. (2016). Validity of PROMIS physical function measures in diverse clinical samples. Journal of Clinical Epidemiology, 73, 112-118. https://doi.org/10.1016/j.jclinepi.2015.08.039

Schottenfeld, D., \& Beebe-Dimmer, J. L. (2006). Multiple primary cancers. In D. Schottenfeld \& J. Fraumeni Jr. (Eds.), Cancer Epidemiology and Prevention (3rd ed., pp. 1269-1280). New York, NY: Oxford University Press.

Sereika, S., \& Engberg, S. (2006). Development of standardized sociodemographic and comorbidity questionnaires. Sigma Theta Tau International Honor Society of Nursing 17th International Nursing Research Congress. Montreal, Quebec: Sigma Theta Tau International Honor Society of Nursing. Retrieved from https://stti.confex.com/stti/congrs06/techprogram/paper_30321.htm

Shapiro, C. L., McCabe, M. S., Syrjala, K. L., Friedman, D., Jacobs, L. A., Ganz, P. A., ... Marcus, A. C. (2009). The LIVESTRONG Survivorship Center of Excellence Network. Journal of Cancer Survivorship, 3(1), 4-11. https://doi.org/10.1007/s11764-008-0076-8

Sherwood, P. R., Given, B. A., Donovan, H., Baum, A., Given, C. W., Bender, C. M., \& Schulz, R. (2008). Guiding research in family care: A new approach to oncology caregiving. Psycho-Oncology, 17, 986-996. https://doi.org/10.1002/pon.1314

Sherwood, P., Ren, D., Given, C. W., Donovan, H., Weimer, J., Belcher, S., \& Given, B. (2016). The impact of caregivers' depression on their physical health. Psycho-Oncology, 25(Supplement S3; Abstract 70), 24. https://doi.org/10.1002/pon.4272

Socha, A., Cooper, C. A., \& McCord, D. M. (2010). Confirmatory factor analysis of the M5-50: An implementation of the International Personality Item Pool item set. Psychological Assessment, 22(1), 43=49. https://doi.org/10.1037/a0017371

Spitzer, R. L., Kroenke, K., Williams, J. B. W., \& Lo, B. (2006). A brief measure for assessing generalized anxiety disorder: The GAD-7. Archives of Internal Medicine, 166(10), 10921097. https://doi.org/10.1001/archinte.166.10.1092

Stein, K. D., Syrjala, K. L., \& Andrykowski, M. A. (2008). Physical and psychological long-term and late effects of cancer. Cancer, 112(11 SUPPL.), 2577-2592.

https://doi.org/10.1002/cncr.23448

Syrjala, K. L., \& Yi, J. C. (2018). Overview of psychological issues in the adult cancer survivor. 
Retrieved April 13, 2018, from https://www-uptodatecom.pitt.idm.oclc.org/contents/overview-of-psychosocial-issues-in-the-adult-cancersurvivor? search=Overview of psychosocial issues in the adult cancer survivor\&source=search_result\&selectedTitle=1 150\&usage_type=default\&display_rank=1

Tabachnick, B. G., \& Fidell, L. S. (2013). Using multivariate statistics (6th ed.). Boston, MA: Pearson Education Limited.

The Centers for Disease Control and Prevention. (2018). Current cigarette smoking among adults in the United States. Retrieved June 4, 2018, from http://www.cdc.gov/tobacco/data_statistics/fact_sheets/adult_data/cig_smoking/

Thewes, B., Butow, P., Zachariae, R., Christensen, S., Simard, S., \& Gotay, C. (2012). Fear of cancer recurrence: A systematic literature review of self-report measures. PsychoOncology, 21(6), 571-587. https://doi.org/10.1002/pon.2070

Thong, M. S. Y., Mols, F., Verhoeven, R. H. A., Liu, L., Andrykowski, M. A., Roukema, J. A., \& Van De Poll-Franse, L. V. (2013). Multiple primary cancer survivors have poorer health status and well-being than single primary cancer survivors: A study from the populationbased PROFILES registry. Psycho-Oncology, 22, 1834-1842. https://doi.org/10.1002/pon.3227

Underwood, J. M., Townsend, J. S., Stewart, S. L., Buchannan, N., Ekwueme, D. U., Hawkins, N. a, ... Fairley, T. L. (2012). Surveillance of demographic characteristics and health behaviors among adult cancer survivors--Behavioral Risk Factor Surveillance System, United States, 2009. Morbidity and Mortality Weekly Report. Surveillance Summaries (Washington, D.C. : 2002), 61(1), 1-23. Retrieved from http://www.ncbi.nlm.nih.gov/pubmed/22258477

University of Pittsburgh Computing Services and Systems Development. (n.d.). Qualtrics survey service. Retrieved February 7, 2017, from http://technology.pitt.edu/service/qualtricssurvey-service

Vinokur, A. D., Price, R. H., \& Caplan, R. D. (1996). Hard times and hurtful partners: How financial strain affects depression and relationship satisfaction of unemployed persons and their spouses. Journal of Personality and Social Psychology, 71(1), 166-179. https://doi.org/10.1037/0022-3514.71.1.166

Vodermaier, A., Linden, W., \& Siu, C. (2009). Screening for emotional distress in cancer patients: A systematic review of assessment Instruments. Journal of the National Cancer Institute, 101(21), 1464-1488. https://doi.org/10.1093/jnci/djp336

Weiss, D. S. (2007). The Impact of Event Scale: Revised. In J. P. Wilson \& C. C. So-Kum Tang (Eds.), International and Cultural Psychology: Cross-Cultural Assessment of Psychological Trauma and PTSD (pp. 219-238). Boston, MA: Springer.

Weiss, D. S., \& Marmar, C. R. (1997). The Impact of Event Scale-Revised: Initial studies of the original Impact of Event Scale. In J. P. Wilson \& T. M. Keane (Eds.), Assessing Psychological Trauma and PTSD: A Practitioner's Handbook (pp. 399-411). New York: The Guilford Press. 
Whooley, M. A., Avins, A. L., Miranda, J., \& Browner, W. S. (1997). Case-finding instruments for depression: Two questions are as good as many. Journal of General Internal Medicine, 12(7), 439-445. https://doi.org/10.1046/j.1525-1497.1997.00076.x

Yeom, H. y.-A., Choi, M., Belyea, M., \& Fleury, J. (2011). Psychometric evaluation of the Index of Self-Regulation. Western Journal of Nursing Research, 33(2), 268-285. https://doi.org/10.1177/0193945910378854

Yoshinaga, a, Sasaki, S., \& Tsugane, S. (2001). Sensitivity of self-reports of cancer in a population-based prospective study: JPHC Study Cohort I. Journal of Clinical Epidemiology, 54(7), 741-746. Retrieved from http://www.ncbi.nlm.nih.gov/pubmed/11438416 